\title{
A SECURITY BASED APPROACH TO COMPOSITE POWER SYSTEM RELIABILITY EVALUATION
}

\author{
A Thesis \\ Submitted to the College of Graduate Studies and Research \\ in Partial Fulfilment of the Requirements \\ for the Degree of \\ Doctor of Philosophy \\ in the \\ Department of Electrical Engineering \\ University of Saskatchewan \\ $\underbrace{}_{\substack{\text { Max } \\ \text { Mar. } 5 / \text { y }}}$
}

by

MD. EASIN KHAN

Saskatoon, Saskatchewan

January 1991

Copyright (C) 1991 MD. EASIN KHAN

The author claims copyright. Use shall not be made of the material contained herein without proper acknowledgement, as indicated on the copyright page. 
To

My Beloved Parents,

Parents-in-Law,

Nina and Nisath. 
UNIVERSITY OF SASKATCHEWAN

COLLEGE OF GRADUATE STUDIES AND RESEARCH

\section{Saskatoon}

\section{CERTIFICATION OF THESIS WORK}

We, the undersigned, certify that Md. Easin Khan candidate for the degree of Doctor of Philosophy has presented a thesis with the following title:

"A Security Based Approach To Composite Power System Reliability

Evaluation". We consider that the thesis is acceptable in form and content, and that a satisfactory knowledge of the field covered by the thesis was demonstrated by the candidate through an oral examination held on January 4, 1991.

External Examiner: Dr. Murty P. Bhavaraju Public Service Electric and Gas Company Newark, New Jersey

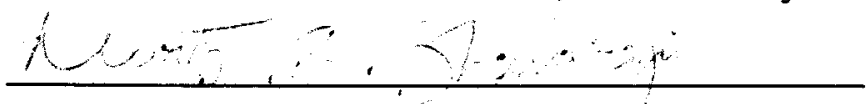

Internal Examiners:

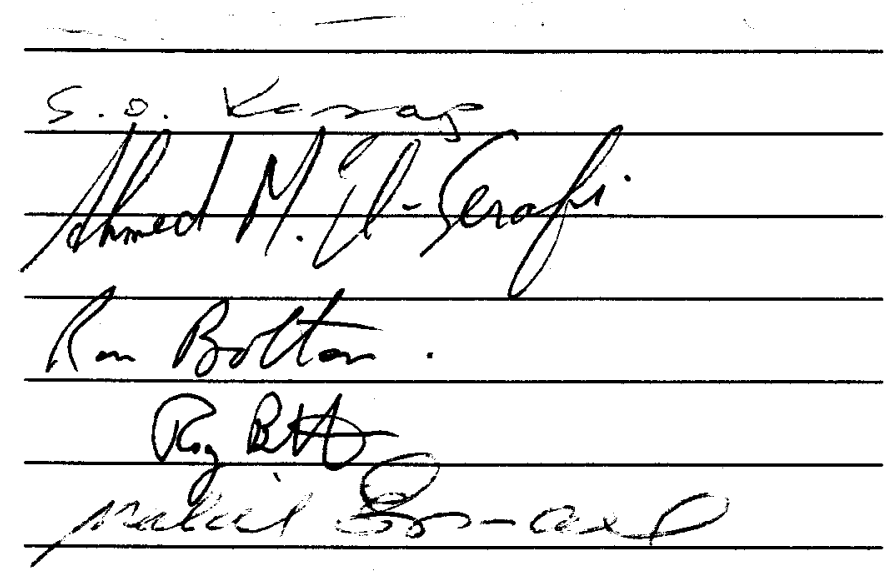

January 4, 1991 


\section{COPYRIGHT}

The author has agreed that the Library, University of Saskatchewan, may make this thesis freely available for inspection. Moreover, the author has agreed that permission for extensive copying of this thesis for scholarly purposes may be granted by the Professor who supervised the thesis work recorded herein or, in his absence, by the Head of the Department or the Dean of the College in which the thesis work was done. It is understood that due recognition will be given to the author of this thesis and to the University of Saskatchewan in any use of the material in this thesis. Copying or publication or any other use of this thesis for financial gain without approval by the University of Saskatchewan and the author's written permission is prohibited.

Requests for permission to copy or to make any other use of the material in this thesis in whole or in part should be addressed to:

Head of the Department of Electrical Engineering

University of Saskatchewan

Saskatoon, Canada S7N 0W0. 


\section{ACKNOWLEDGEMENTS}

The author would like to express his gratitude and appreciation to Dr. Roy Billinton for his guidance and consistent encouragement throughout the course of this work. His advice and assistance in the preparation of this thesis is thankfully acknowledged. The author would especially like to acknowledge that without his many hours of dedicated interest and assistance, the completion of this work would not have been possible at the author's desired time. These simple words fail to convey the author's sincere appreciation for all the help that has been given by him to the author. The author has no words in which to express his respect for Dr. Roy Billinton as an academician, as a teacher and as a person. The author would always remain indebted towards him.

The author takes this opportunity to acknowledge the patience, encouragement and moral support provided by his mother, wife Nina, son Nisath, parents-in-law and all other family members.

Financial assistance provided by the Canadian Commonwealth Scholarship and Fellowship Plan is thankfully acknowledged.

The author thankfully acknowledges the study leave granted by the Bangladesh University of Engineering and Technology, Dhaka. 


\title{
ABSTRACT \\ UNIVERSITY OF SASKATCHEWAN
}

Electrical Engineering Abstract 90 A333

\section{A SECURITY BASED APPROACH TO COMPOSITE POWER SYSTEM RELIABILITY EVALUATION}

\author{
Student: Md. Easin Khan Supervisor: Dr. Roy Billinton \\ Ph.D. Thesis Presented to the \\ College of Graduate Studies and Research
}

January 1991

\begin{abstract}
There is considerable interest in the application of probability methods to composite system reliability evaluation. The problem is extremely complex because of the need to include detailed modelling of both generation and transmission facilities and to consider multiple levels of component failures. Quantitative adequacy assessment of a composite power system is generally done using a contingency enumeration approach which includes the evaluation of contingencies, the classification of these contingencies according to selected failure criteria and the accumulation of adequacy indices. There are several network solution methods presently available depending upon the failure criteria and the intent behind the studies. In this thesis, adequacy indices are calculated using three methods, namely network flow, dc and ac load flow and the importance of utilizing an ac load flow method in composite system reliability analysis is clearly illustrated. The computation time increases tremendously when an ac load flow method is used specifically for a large system where the inclusion of higher level outages cannot be ignored in the calculation of representative indices. In order to reduce the computation time when using ac load flow without sacrificing much accuracy, different approximate
\end{abstract}


methods can be utilized. Adequacy indices are calculated and presented in this thesis using three ranking methods and a new technique designated as the selection method. The CPU times and the accuracy of the methods as compared to the reference values obtained with an exhaustive ac load flow solution are discussed.

The presently available techniques for quantitative reliability evaluation of composite power systems are in the adequacy domain. The most significant quantitative indices in this regard are those which relate to load curtailments and many utilities have concerns in interpreting the expected load curtailment indices. A framework for incorporating the steady-state security considerations in the reliability evaluation of composite power systems is examined and extended in this thesis. The system operating states are quantified using the contingency enumeration method for three different constraint sets. The indices are also calculated by combining the contingency enumeration method and a Monte Carlo simulation approach through the use of hybrid methods to quantify the various system operating states and the results are compared with the analytical values.

This thesis presents a new risk index designated as the Composite System Operating State Risk (CSOSR). This index is defined and its utilization in system expansion and unit commitment in composite generation and transmission systems is illustrated. The concepts presented in this thesis are illustrated numerically using two basic test systems. 


\section{Table of Contents}

COPYRIGHT

ACKNOWLEDGEMENTS

ABSTRACT

Table of Contents

List of Figures

List of Tables

List of Abbreviations

1. INTRODUCTION

1.1. INTRODUCTION

1.2. SCOPE OF THE THESIS

i

ii

iii

v

ix

xii

xvii

2. ADEQUACY EVALUATION OF COMPOSITE POWER SYSTEMS - ENUMERATION APPROACH

2.1. INTRODUCTION

2.2. NETWORK SOLUTION TECHNIQUES

2.2.1. Network Flow Method

2.2.2. DC Load Flow Method

2.2.3. Fast Decoupled Load Flow Method

2.3. SHERMAN-MORRISON CORRECTION 21

2.4. STATE-SPACE MODELS 23

2.4.1. A Single Component Model 24

2.4.2. Model For Independent Overlapping Outages 25

2.5. CORRECTIVE ACTIONS

2.6. ADEQUACY INDICES 28

2.6.1. Load Point Indices $\quad 30$

2.6.2. System Indices 31

2.7. TEST SYSTEMS

2.7.1. The Roy Billinton Test System (RBTS) 33

2.7.2. The IEEE Reliability Test System (IEEE-RTS) 33

2.8. SYSTEM STUDIES

2.8.1. The RBTS

2.8.2. The IEEE-RTS

2.9. CONTINGENCY EVALUATION CUTOFF CRITERIA

41

2.9.1. Contingency Level Cutoff Criteria

43

2.10. APPROXIMATE SOLUTION CONSIDERATIONS

46 
2.11. SUMMARY

3. FAST APPROXIMATE ADEQUACY EVALUATION 50

3.1. INTRODUCTION 50

3.2. ADEQUACY EVALUATION USING RANKING 51

3.2.1. Performance Indices For Ranking $\quad 52$

3.2.2. Ranking Of Outage Contingencies 52

3.2.2.1 Ranking Using Line-Overloads

3.2.2.2 Ranking Using Voltage Deviation

3.2.3. Preparation Of A Contingency Ranked List 56

3.2.4. Ranking Cutoff Criteria 57

3.3. ADEQUACY EVALUATION USING THE SELECTION 58 METHOD

3.3.1. Selection Method For Transmission Line Outages 59

3.4. REFERENCE CASE RESULTS 61

3.5. COMPARISON OF RANKING AND SELECTION METHODS 61 FOR LINE OUTAGES

3.5.1. Using Ranking 61

3.5.2. Using Selection 68

3.6. GENERATOR AND GENERATOR + LINE OUTAGES

3.6.1. Selection Of Generator Outages 71

3.6.2. Selection Of Generator And Line Outages 73

3.6.3. Study Results 76

3.7. SUMMARY $\quad 79$

4. PROBABILISTIC SECURITY ASSESSMENT OF A

COMPOSITE POWER SYSTEM

4.1. INTRODUCTION 84

4.2. SECURITY CONSIDERATIONS

4.2.1. System Model Including Security Considerations 85

4.2.2. Modified System Model 88

4.2.3. Security Constraints 90

4.3. MATHEMATICAL MODEL 92

4.3.1. Selection Method 92

4.3.2. Method For Calculating Changes In Voltage And Angle Vectors 92

4.3.3. Linear Programming Model For Load Shedding And Generation 96

Rescheduling

4.4. LINEAR PROGRAMMING ALGORITHMS TO SOLVE THE 97 MODEL

4.4.1. Primal Simplex Algorithm With Bounded Variable 99

4.4.2. Dual Simplex Method For Bounded Variables 102

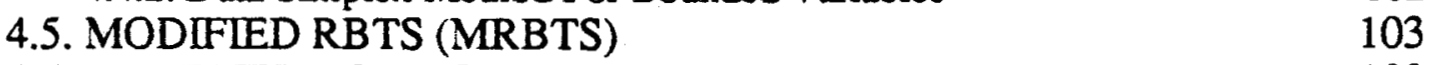

4.6. SPLIT NETWORK SITUATION 103

4.6.1. Approximate Method For Split Network Sinuation Solution 105

4.7. FLOW CHART FOR DETECTING THE STATES 108

4.8. STUDY RESULTS 110 
4.8.1. MRBTS Results

4.8.2. IEEE-RTS Results

4.9. EFFECT OF LOAD VARIATION AND ANNUAL INDICES 112

4.10. CONTINGENCY SORTING

4.11. SUMMARY

5. VOLTAGE PROBLEM CONSIDERATIONS IN THE

RELIABILITY ASSESSMENT OF COMPOSITE POWER SYSTEMS

5.1. INTRODUCTION

5.2. CONSIDERATION OF VOLTAGE PROBLEMS

5.3. CONSIDERATION OF GENERATOR MVAR LIMITS

5.4. REACTIVE LOAD CURTAILMENT BY THE LP MODEL

5.5. STUDY RESULTS

5.5.1. Results For The MRBTS

135

5.5.2. Results For The IEEE-RTS

136

5.6. TOTAL CONSTRAINT SET AND ANNUAL INDICES 137

5.7. SORTING

5.8. SUMMARY

6. A HYBRID MODEL FOR RELIABILITY EVALUATION OF

COMPOSITE POWER SYSTEMS

6.1. INTRODUCTION

6.2. SIMULATION METHODS FOR POWER SYSTEM RELIABILITY EVALUATION

6.3. HYBRID MODELS

6.3.1. Method 1

6.3.2. Discussion Of The Results - Method 1

151

6.3.3. Method 2

153

6.4. STOPPING RULE

6.5. VARIANCE REDUCTION TECHNIQUES

6.5.1. Antithetic Variates

6.5.2. Stratification After Sampling

179

6.5.3. Results With The VRT

180

181

6.6. ANNUAL INDICES

185

6.7. SUMMARY

188

190 CONCEPTS FOR SYSTEM PLANNING

7.1. INTRODUCTION

7.2. EXPANSION PLANNING IN THE MRBTS 191

7.2.1. Generation Capacity Addition

7.2.2. Transmission Line Additions

196

7.2.3. Composite Generation And Transmission System Reinforcement 201

7.3. EXPANSION PLANNING IN THE IEEE-RTS

204

7.3.1. Generation Capacity Addition 
7.3.2. Transmission Line Additions

7.3.3. Composite Generation And Transmission System Reinforcement 215

7.4. SUMMARY

8. SECURITY EVALUATION USING CAPACITY TABLE 219 APPROACH

8.1. INTRODUCTION

8.2. ANALYSIS OF DIFFERENT STATES USING A CAPACITY

OUTAGE PROBABILITY TABLE

8.2.1. Capacity Outage Probability Table

8.2.2. Use Of A Capacity Table For Calculating The Indices

8.3. LEAD TIME AND ITS EFFECT ON THE OPERATING STATES

8.4. SPINNING RESERVE ASSESSMENT IN HLI

8.4.1. Unit Commitment In HLI

8.4.2. Results For The MRBTS

8.4.3. Results For The IEEE-RTS

8.5. SUMMARY

9. CONCLUSIONS

Appendix A. Data of the 6 bus RBTS

Appendix B. Data of the IEEE-RTS 


\section{List of Figures}

Figure 1.1: Hierarchical levels for reliability analysis 3

Figure 2.1: Contingency enumeration approach 13

Figure 2.2: Single component two state model $\quad 24$

Figure 2.3: Model for two independent overlapping outages 25

Figure 2.4: Single line diagram of the RBTS 34

Figure 2.5: Single line diagram of the IEEE-RTS 35

Figure 3.1: Flow chart for the selection of line contingencies 62

Figure 3.2: Percent of contingencies captured, tested and the CPU time 63 as a function of $\mathrm{N}$ for the RBTS as a percentage of the reference case

Figure 3.3: Percent of contingencies captured, tested and the CPU time as a function of $\mathrm{N}$ for the IEEE-RTS as a percentage of the reference case

Figure 3.4: Flow chart for the selection of generator contingencies

Figure 3.5: Flow chart for the selection of generator+line contingencies

Figure 4.1: System operating states

Figure 4.2: Modified diagram for system operating states

Figure 4.3: Modified RBTS

Figure 4.4: Split network situation

Figure 4.5: Flow chart for detecting different operating states

Figure 4.6: Multi-step load model

109

Figure 6.1: Flow chart for hybrid Method 1

Figure 6.2: Probability and frequency of the normal state with number of samples using Method 1 - MRBTS

Figure 6.3: Probability and frequency of the alert state with number of samples using Method 1 - MRBTS

Figure 6.4: Probability and frequency of the emergency state with number of samples using Method 1 - MRBTS

Figure 6.5: Probability and frequency of the extreme emergency state with number of samples using Method 1 - MRBTS

Figure 6.6: Probability and frequency of the no problem with number of samples using Method 1 - MRBTS

Figure 6.7: Probability and frequency of the normal state with number of samples using Method 1 - IEEE-RTS 
Figure 6.8: Probability and frequency of the alert state with number of 160 samples using Method 1 - IEEE-RTS

Figure 6.9: Probability and frequency of the emergency state with number of samples using Method 1 - IEEE-RTS

Figure 6.10: Probability and frequency of the extreme emergency state with number of samples using Method 1 - IEEE-RTS

Figure 6.11: Probability and frequency of the no problem with number of samples using Method 1 - IEEE-RTS

Figure 6.12: Flow chart for hybrid Method 2

Figure 6.13: Probability and frequency of the normal state with

Figure 6.14: Probability and frequency of the alert state with number of samples using Method 2 - MRBTS

Figure 6.15: Probability and frequency of the emergency state with number of samples using Method 2 - MRBTS

Figure 6.16: Probability and frequency of the extreme emergency state with number of samples using Method 2 - MRBTS

Figure 6.17: Probability and frequency of the no problem with number of samples using Method 2 - MRBTS

Figure 6.18: Probability and frequency of the normal state with number of samples using Method 2 - IEEE-RTS

Figure 6.19: Probability and frequency of the alert state with number of samples using Method 2 - IEEE-RTS

Figure 6.20: Probability and frequency of the emergency state with number of samples using Method 2 - IEEE-RTS

Figure 6.21: Probability and frequency of the extreme emergency state with number of samples using Method 2 - IEEE-RTS

Figure 6.22: Probability and frequency of the no problem with number of samples using Method 2 - IEEE-RTS

Figure 7.1: CSOSR variation with peak load increment - MRBTS

Figure 7.2: CSOSR variation with addition of generation at Bus 1 MRBTS

Figure 7.3: CSOSR variation with addition of generation at Bus 2 MRBTS

Figure 7.4: Comparison of addition of generation at Bus 1 and 2MRBTS

Figure 7.5: CSOSR variation with addition of transmission line MRBTS

Figure 7.6: CSOSR variation with addition of transmission line MRBTS

Figure 7.7: CSOSR variation with transmission line rating - MRBTS 201

Figure 7.8: CSOSR variation with load increment at 1.35 times of 202 transmission line rating - MRBTS 
Figure 7.9: CSOSR variation for composite system - MRBTS 205

Figure 7.10: CSOSR variation with addition of generation at Bus 1 - 207 IEEE-RTS

Figure 7.11: CSOSR variation with addition of generation at Bus 2 - 208 IEEE-RTS

Figure 7.12: CSOSR variation with addition of generation at Bus 7 - 209 IEEE-RTS

Figure 7.13: CSOSR variation with addition of generation at Bus $15-210$ IEEE-RTS

Figure 7.14: CSOSR variation with addition of generation at Bus 16 - 211 IEEE-RTS

Figure 7.15: CSOSR variation with addition of generation at Bus 23 - $\quad 212$ IEEE-RTS

Figure 7.16: CSOSR variation with addition of transmission line in the 214 south region - IEEE-RTS

Figure 7.17: CSOSR variation with addition of transmission line in the 215 north region - IEEE-RTS

Figure 7.18: CSOSR variation with transmission line rating - IEEE- 216 RTS

Figure 7.19: CSOSR variation for composite system - IEEE-RTS

Figure 8.1: Probability of the normal and alert states - MRBTS

Figure 8.2: Probability of the emergency and extreme emergency states - MRBTS

Figure 8.3: Probability of the normal and alert states - IEEE-RTS

Figure 8.4: Probability of the emergency and extreme emergency states - IEEE-RTS

Figure 8.5: Variation of risks with the lead time - IEEE-RTS 


\section{List of Tables}

Table 2.1: Recommended failure criterion for different solution 28 techniques

Table 2.2: Total number of different contingencies for the RBTS 37

Table 2.3: Probability and frequency of failure for up to second level 37 contingencies - RBTS

Table 2.4: Load point indices for up to second level contingencies - 38 RBTS

Table 2.5: System indices for up to second level contingencies - RBTS 39

Table 2.6: Probability and frequency of failure for up to second level 41 contingencies - IEEE-RTS

Table 2.7: Expected load and energy curtailed for up to second level 42 contingencies - IEEE-RTS

Table 2.8: Total number of different contingencies for up to second level - IEEE-RTS

Table 2.9: System indices up to second level contingencies - IEEE- 44 RTS

Table 2.10: Total number of possible states for the two test systems 44

Table 2.11: Sum of the probabilities for different contingency levels 45 for the two test systems

Table 2.12: CPU time required for solution of the two test systems at various contingency levels (on MicroVax 3600 digital computer)

Table 2.13: Number of contingencies creating system problems for the 48 two test systems

Table 3.1: Comparison of CPU time and capture ratio - RBTS

Table 3.2: Comparison of CPU time and capture ratio - IEEE-RTS 66

Table 3.3: Comparison of probability and frequency of failure - IEEE- 67 RTS

Table 3.4: Comparison of load and energy curtailment indices - IEEE- 68 RTS

Table 3.5: Comparison of different system indices - IEEE-RTS

Table 3.6: Number of generator contingencies studied by selection

Table 3.7: Increment in CPU time and capture ratio of the reference method over that of the selection method for generator contingencies 
Table 5.13: Decoupled load flow results with modified voltages 134

Table 5.14: Decoupled load flow results with modified voltages and $Q \quad 135$

Table 5.15: Probability and frequency of different operating states - 136 MRBTS, considering only voltage problems

Table 5.16: Probability and frequency of different operating states - 137 IEEE-RTS, considering only voltage problems

Table 5.17: Effect of Load variation on the probability of different 138 states - MRBTS, considering only voltage problems

Table 5.18: Effect of load variation on the frequency of different states 138 - MRBTS, considering only voltage problems

Table 5.19: Effect of load variation on the probability of different states - IEEE-RTS, considering only voltage problems

Table 5.20: Effect of load variation on the frequency of different states - IEEE-RTS, considering only voltage problems

Table 5.21: Probability and frequency of different operating states MRBTS, considering total constraint set

Table 5.22: Probability and frequency of different operating states IEEE-RTS, considering total constraint set

Table 5.23: Effect of load variation on the probability of different states - MRBTS, considering total constraint set

Table 5.24: Effect of load variation on the frequency of different states - MRBTS, considering total constraint set

Table 5.25: Effect of load variation on the probability of different states - IEEE-RTS, considering total constraint set

Table 5.26: Effect of load variation on the frequency of different states - IEEE-RTS, considering total constraint set

Table 5.27: Effect of voltage setting on the probability of different states - IEEE-RTS, considering total constraint set

Table 5.28: Effect of voltage setting on the frequency of different states - IEEE-RTS, considering total constraint set

Table 5.29: Comparison of contingency considered and computation time with and without sorting

Table 6.1: Outages considered using enumeration with generator 1 outage by sampling

Table 6.2: Number of samples for different outage levels - MRBTS

Table 6.3: Number of samples for different outage levels - IEEE-RTS

Table 6.4: Probability and frequency of different states for various $\alpha$ MRBTS

Table 6.5: Probability and frequency of different states for various $\alpha$ IEEE-RTS

Table 6.6: Probabilities of different stratum for the two test systems

Table 6.7: Probability and frequency of different states without and with VRT - MRBTS 
Table 6.8: Number of samples in different system states from different stratum - MRBTS

Table 6.9: Variance of estimation and CPU time for the two test systems

Table 6.10: Probability and frequency of different states without and with VRT - IEEE-RTS

Table 6.11: Number of samples in different system states from different stratum - IEEE-RTS

Table 6.12: Probability of different load levels using simulation

Table 6.13: Contribution of different load levels to state probability MRBTS

Table 6.14: Contribution of different load levels to state frequency MRBTS

Table 6.15: Contribution of different load levels to state probability IEEE-RTS

Table 6.16: Contribution of different load levels to state frequency IEEE-RTS

Table 7.1: Additional generating units for the MRBTS

Table 7.2: CSOSR for different line additions

Table 7.3: Comparison of facilities addition

Table 7.4: Additional generating units for the IEEE-RTS

Table 8.1: Capacity outage probability table at bus 1 - MRBTS

Table 8.2: Capacity outage probability table at bus 2 - MRBTS

Table 8.3: Probability and frequency of different operating states MRBTS

Table 8.4: Probability and frequency of different operating states IEEE-RTS

Table 8.5: Priority loading order list of generating units - MRBTS

Table 8.6: Number of generators required for different lead time with fixed load level - MRBTS

Table 8.7: Number of generators required for different peak load with fixed lead time - MRBTS

Table 8.8: Effect of variation of load on the probability of different states with fixed lead time - MRBTS

Table 8.9: CSOSR at the 166.5 MW load level - MRBTS

Table 8.10: Priority loading order list of generating units - IEEE-RTS

Table 8.11: Number of generators required for different lead time with fixed load level - IEEE-RTS

Table 8.12: Number of generators required for different peak load with fixed lead time - IEEE-RTS

Table 8.13: Effect of load variation on the probability of different states with fixed lead time - IEEE-RTS

Table 8.14: CSOSR at the $2850 \mathrm{MW}$ load level - IEEE-RTS 
Table A.1: Bus data

Table A.2: Line data

Table A.3: Generator data

Table B.1: Bus data

Table B.3: Generator data 


\section{List of Abbreviations}

ACLF

ADLC

AEC

ALC

ANHLC

ANLC

ANVV

BPII

BPECI

BPSAMC

BPSD

CSOSR

DCLF

HL1

HL2

HL3

IEEE-RTS

LOEE

LOLE

MBPECI

MEC

MLC

MRBTS

$N B$

NF

$N L$

$P I$

RBTS

SM

SI

VRT ac Load Flow

Average Duration Of Load Curtailment Per Load Point

Average Energy Curtailment Per Load Point

Average Load Curtailment Per Load Point

Average Number Of Hours Of Load Curtailment Per Load Point

Average Number Of Load Curtailment Per Load Point

Average Number Of Voltage Violation Per Load Point

Bulk Power Interruption Index

Bulk Power Energy Curtailment Index

Bulk Power Supply Average MW Curtailment

Bulk Power Supply Disturbances

Composite System Operating State Risk

dc Load Flow

Hierarchical Level 1

Hierarchical Level 2

Hierarchical Level 3

IEEE-Reliability Test System

Loss Of Energy Expectation

Loss Of Load Expectation

Modified Bulk Power Energy Curtailment Index

Maximum Energy Curtailed

Maximum Load Curtailed

Modified Roy Billinton Test System

Number Of Buses

Network Flow

Number Of Lines

Performance Index

Roy Billinton Test System

Sherman-Morrison

Severity Index

Variance Reduction Technique 


\section{INTRODUCTION}

\subsection{INTRODUCTION}

The basic objective of an electric power utility is to supply electrical energy to its consumers as economically as possible and with an acceptable degree of reliability and quality. Modern society, because of its pattern and working habits, has come to expect that the supply should be continuously available on demand. It is not, however, feasible economically and technically to attempt to design a power system with one hundred percent reliability. Power system engineers have always attempted to achieve the highest possible reliability within economic constraints and therefore reliability evaluation of the system is an important activity. The word "reliability" has a wide range of meanings and when used in a power system context, it is generally defined as the concern regarding the ability of the power system to provide an adequate supply of electrical energy [1]. A simple but reasonable subdivision of the term "system reliability" can be made by considering two basic and fundamental aspects of the system, namely adequacy and security $[2,3,4]$.

Adequacy and security of a power system have been major concerns of the power system planner for many years. System adequacy is defined as the capability of the system to supply its load taking into consideration transmission constraints and scheduled and unscheduled outages of generators and transmission facilities. System security is defined as the ability of the power system to withstand disturbances arising from faults or the unscheduled removal of bulk power supply equipment(s). Therefore adequacy encompasses the steady state post outage analysis of the bulk power system while security encompasses the analysis of dynamic conditions.

An electric power system generates electrical energy at its generating stations and supplies it to the individual customers through a suitable transmission and distribution 
network. The basic techniques for reliability assessment can, therefore, be subdivided in terms of their application to segments of a complete power system. These segments are called "functional zones". The three basic functional zones for the purpose of planning, organization and/or analysis are: generation, transmission and distribution. These functional zones can be combined to form a series of hierarchical levels for the purpose of conducting system reliability analysis. Reliability assessment at the different hierarchical levels and functional zones has undergone continuous development and application since the 1930s. These developments can be seen from the bibliographies [5]- [9] published in the IEEE which contain nearly 700 individual papers on reliability assessment of power systems. The functional zones and hierarchical levels are shown in Figure 1.1. Hierarchical level two (HLII) consists of both generation and transmission facilities and is also known as the composite or bulk system. Composite system reliability evaluation techniques are therefore concerned with the total problem of assessing the generation and transmission facilities in regard to their ability to supply adequate, dependable and suitable electrical energy to the major customer load points. This research work is restricted to reliability assessment of composite power systems.

Composite generation and transmission system reliability evaluation came under active investigation in North America and Europe in the 1960's. Reference [10] is an early example of consideration of the combined problem of generation and transmission in reliability evaluation. The term 'composite system reliability evaluation' was proposed in 1969 by Billinton [11]. This approach included a more complete system representation of the form used in load flow analysis. This technique utilizes a quality of service rather than a simple continuity of service criterion. A system failure is charged if the supply at a major transmission bus does not meet predetermined voltage standards and/or allowable equipment loadings. In this approach, adequacy evaluation of a composite generation and transmission system involves the simulation and computation of the system conditions for each possible outage condition in the system in order to determine the voltage violations, line and generator overloads, violation of generator MVAr limits etc.. During the late 1960's, two independent streams of activity [11]- [16] started in North America and Europe for the reliability evaluation of composite power systems. These two approaches are known as contingency enumeration and Monte Carlo 


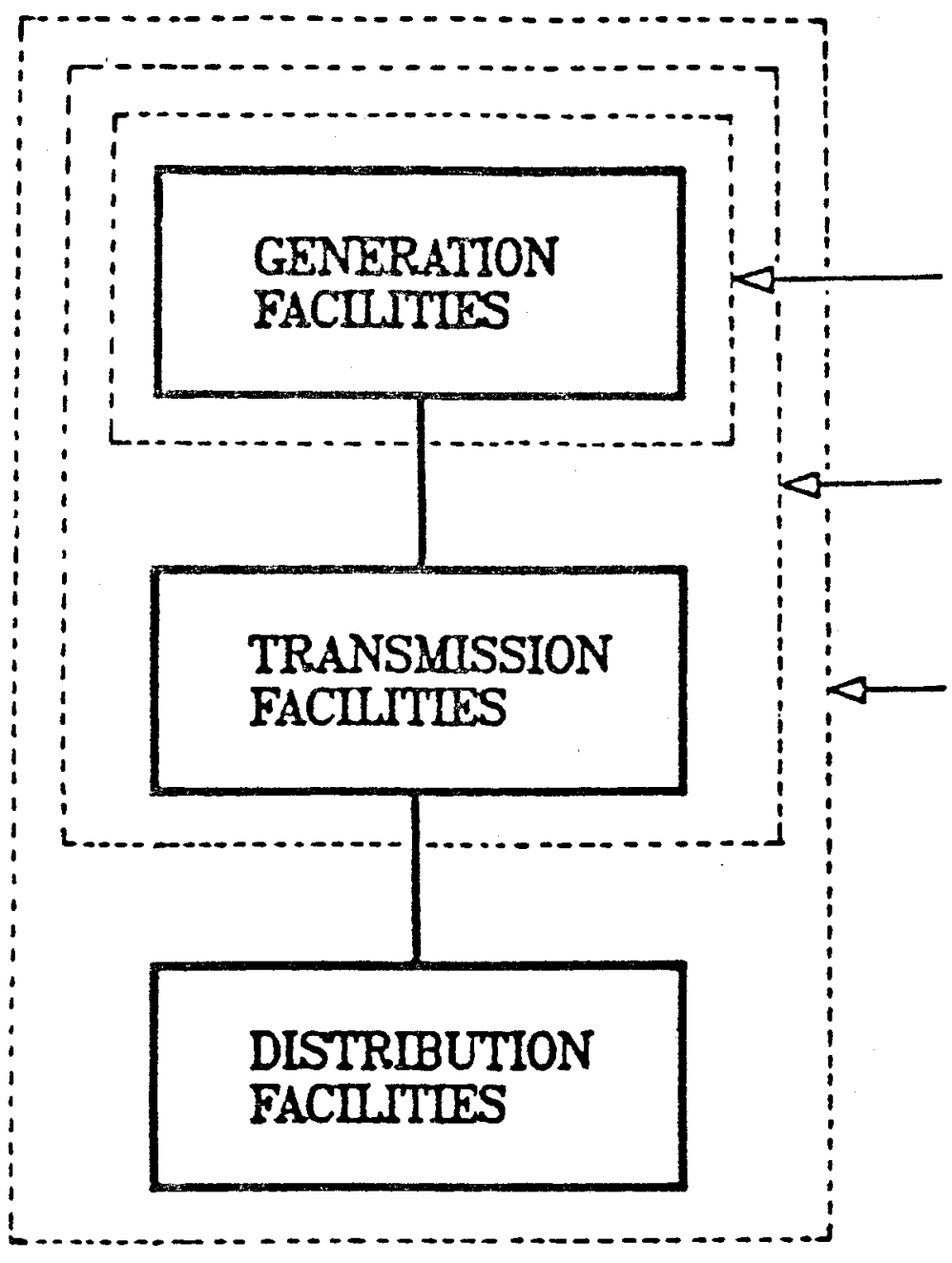

Hierarchical Level II

Hierarchical Level III

Figure 1.1: Hierarchical levels for reliability analysis

simulation respectively. These two methods of reliability evaluation are fundamentally different and may be initiated by the requirements to represent generating elements in HLI studies. The French and Italian systems have significant hydro resources and utilize Monte-Carlo simulation whereas the precedents derived from North American generating capability reliability models tended to influence the enumeration approach in North America. Further research work in both the area of simulation [17]- [26] and contingency enumeration [27]- [37] have been published in the literature. A comparison between contingency enumeration and simulation techniques was published in 1985 
using the IEEE Reliability Test System [38] and is described in Reference [39]. These comparisons indicate the conceptual differences in modelling and problem perception and allows a better understanding of the merits and demerits of the two approaches. A series of selected important papers on reliability assessment of electric power systems are presented in Reference [40].

Reliability assessment at HLII is a complex task and there is no single accepted procedure. The computation time requirements to analyze a practical power system is quite significant since for determining the effect of unscheduled or unexpected disturbances or contingencies on the power system, computer simulation e.g. load flows are performed for each of the contingencies of interest. The purpose of this simulation is to determine

1. those contingencies that cause limit violation(s) and

2. the associated limit violation(s).

There is a wide variation both in terms of techniques utilized to analyze the system and the quantitative indices created to reflect the reliability of the system.

A contingency is a sudden change in a power system due to unscheduled outages of equipment such as generators and transmission lines, sudden and large changes in the load, and the occurrence of equipment faults. It is important to determine if the system is able to withstand disturbances without violating any system constraints when the system is initially operating in its normal state. This problem is referred to as security analysis. There are two types of security analysis: transient/dynamic and steady-state. Transient/dynamic stability assessment consists of determining if the system oscillations following an outage or a fault will cause loss of synchronism among generators. The objective of steady-state security analysis is to determine whether, following the occurrence of a contingency, there exists a new steady-state secure operating point where the perturbed power system will settle after the dynamic oscillations have damped out. This research work considers steady-state assessment of the security problem in composite system reliability evaluation. 
A contingency may or may not result in the violation of some of the network constraints. If the normal system passes all of the contingency tests, it is said to be operating in a secure state and no further action is taken. If the normal system fails to pass any one of the contingency tests, it is said to be operating in an insecure state and the particular contingency and associated limit violations are noted. When the system is operating in the insecure state, it may be possible to execute a preventive control action aimed at bringing the system into the acceptable operating state. The approaches to preventive control are:

1. modify real power flows by rescheduling real power generation, resetting phase shifting transformer, etc.

2. modify reactive power flows by rescheduling reactive power generation, and by using shunt capacitors and tap changing transformers

3. change the network topology via switching action, etc..

Exhaustive power flow simulation should be performed in order to determine the effect of contingencies on the network. However, this is not computationally feasible for a large system due to the requirement of large computing time. All possible contingencies $[41,42]$ do not create system problems and therefore it is not necessary to solve all possible contingencies by an actual ac load flow analysis. An approximate method can be used to determine the list of contingencies which create system problems and a detailed investigation of these contingencies can be conducted in further studies. The approximate method must have two main properties to be useful. The computational burden for the approximate method with the subsequent ac analysis of the selected contingencies must be less than that for ac analysis of all the contingencies. This can be measured by the ratio of the execution times for the contingency selection and the time required for a full ac load flow analysis. The second desirable property is that the selection should be accurate in the sense that no contingencies which contribute to the system risk are overlooked. Unfortunately, none of the available methods can attain this second desirable property. At best, they can provide a set of contingencies containing most of the cases causing system failure. Some severe contingencies may be omitted and some that are not severe may be included. An increase in accuracy can be obtained only at the cost of an increase in execution time. 
There are two classes of approximate methods which have evolved over the last twenty years: screening and sensitivity-based ranking methods. The sensitivity-based ranking methods [43]- [52] do not identify or solve for specific system conditions. Rather, they quantify the severity of each outages by explicitly calculating a scalar value called a "performance index" by which all contingencies can be ranked. The methods, however, are not completely reliable since they are prone to 'masking errors'. Specifically, a contingency having a few severe violations can be ranked equally with one having many minor violations or even worse, with one without violations. The screening methods [53]- [62], though more demanding in computer resources, permit the identification of actual violations/major shifts and, therefore avoid masking errors.

Reference [4], published in 1978, discusses the two aspects of reliability namely adequacy and security. It should be appreciated that virtually all the basic probabilistic indices are derived in the adequacy domain. Security indices expressed in probabilistic terms are still largely undeveloped [40]. References [63]- [65] present an overview of the major concepts debated by experts in the field of HLII adequacy assessment. The methods of reliability evaluation, goals of the assessment and the conflicting opinions between American and European utility planners are discussed in the above references. There is still considerable developmental work being done in composite system reliability evaluation [66]. The concerns of many power utilities related to composite system reliability evaluation was documented in Reference [65] and this reference also proposed a future frame work for composite system reliability evaluation.

\subsection{SCOPE OF THE THESIS}

There is considerable interest in the application of probability methods in composite system reliability assessment. The problem is extremely complex because of the need to include detailed modelling of both generation and transmission facilities and to consider multiple levels of component failures. Composite system reliability assessment involves both generation and transmission outages and failure is defined when there is no solution which satisfies a defined set of constraints. The probability of different unacceptable conditions can be computed as indices of adequacy and security using the probabilities of contingencies that could cause these conditions. A direct approach to this problem 
requires a full ac load flow analysis for each contingency, followed by a check of the limit violations that are considered as a system problem in the studies. A completely exhaustive method is not computationally feasible, especially for large power systems. Since all the possible contingencies do not create system problems, a contingency set can be constructed which contains those contingencies that yield constraint violations. Therefore, a computationally efficient computer algorithm is needed that can identify those contingencies that result in constraint violations without performing exhaustive power flow simulations.

The presently available techniques and related important topics of composite system reliability evaluation were recently addressed in an IEEE Power Engineering Society tutorial [67] and are summarized in Reference [65]. All the presently available techniques for quantitative reliability evaluation of composite power systems, as noted in Reference [67], are in the adequacy domain. There are a number of different computer programs available for composite system adequacy evaluation. These programs are briefly described in Reference [68], including a list of the calculated indices and the factors involved in the assessment. As can be seen from Reference [67], the most significant quantitative indices in composite system adequacy assessment are those which relate to load curtailment at both the individual bus and overall system levels. Many utilities have difficulty in interpreting the expected load curtailment indices as the existing models are based on adequacy analysis and in many cases do not consider realistic operating conditions. These concerns were expressed in response to a survey conducted as part of an EPRI project and are summarized in the project report [69]. This survey also indicated that security considerations are important issues in composite system reliability evaluation. In response to the stated utility concerns, a framework for incorporating security considerations was proposed in References $[65,69]$. This framework will be examined and extended in this research.

$$
\text { is }
$$

The two most popular approaches, as noted earlier, in the computation of composite system reliability indices for adequacy and security analysis are: contingency enumeration method and Monte Carlo simulation method. The state or contingency enumeration approach [70] includes the systematic selection and evaluation of 
contingencies, the classification of each contingency according to specified failure criteria and the accumulation of reliability indices. In order to compute annual composite system reliability indices, it is necessary to repeat the calculations for several conditions representing different seasons, major maintenance periods, period with different transfer conditions and different load levels. Computation time requirements tend to limit the number of system conditions for large scale systems. The use of the Monte Carlo simulation method allows detailed modelling of precontingency conditions, generation and transmission outages and operating practices $[17,71]$. Parameters that define a system operating state, such as load and the state of generation and transmission components, are selected by random sampling in accordance with the probability distributions of the parameters. The selected operating state is tested and the outcomes evaluated according to one or more failure criteria. A key feature of the Monte Carlo simulation method is the ability to sample events obeying any probability distribution. The contingency enumeration method and the Monte Carlo simulation method are combined through the use of hybrid methods in this research work to quantify the various operating states [72] and the results are compared with the analytical results.

Probabilistic indices for different system operating states are quantified using contingency enumeration as well as using the hybrid approaches. The system states are identified using approximate selection methods for line, generator and combination of line and generator outages. In those events when any line overload or voltage deviation problems are detected, the first objective is to remove the equipment operating constraints without load curtailment by taking corrective action(s). If these actions are not successful, load is curtailed from different buses. The corrective actions are included in this thesis using linear programming methods. The objective of this research work in this regard is, therefore, the development and use of techniques to quantify the various system states associated with recognizing power system security considerations in the reliability evaluation of composite systems. 
Outline of the Thesis

Extensive research work in the area of composite system adequacy evaluation has been done at the University of Saskatchewan by Billinton et al $[70,73,74,75]$. As a result, a digital computer program COMREL(COMposite systems RELiability evaluation) for adequacy assessment of a network has been developed in FORTRAN-77. The program COMREL is equipped with both a fast selection [70] technique using a simple linear model of an electrical network and a detailed solution approach using the fast decoupled ac load flow approach $[73,74]$. In the contingency enumeration approach $[47,68,76]$, used in COMREL, each contingency is tested to determine whether or not it causes an immediate system problem. If it does not, a new contingency is considered and tested. The occurrence of a system problem may by itself be recorded as a failure. It may, however, be possible to eliminate a system problem in many cases by corrective action. A failure is therefore recorded when corrective action, short of curtailing consumer loads, is insufficient to eliminate the system problem. The severity of a system problem may be assessed by computing the amount and location of load curtailment necessary to eliminate the problem.

There are several techniques available for evaluating each contingency. The actual selection depends on the accepted failure criteria and the intent behind the studies. Adequacy indices are calculated in Chapter 2 using the network [77], dc [78] and ac $[60,79]$ load flow methods. Two test systems are utilized throughout the thesis and a brief description of these systems is included in this chapter. The Sherman-Morrison correction formula [80] is used to adjust the base case solution to efficiently represent the line outages instead of rebuilding and refactorizing the system admittance matrices for each line contingency. The algorithm is applicable to both dc and decoupled ac load flow methods. Brief descriptions of the three solution methods together with the application of the Sherman-Morrison correction formula to dc and ac load flow methods are provided in Chapter 2. The adequacy indices calculated using the three methods can be grouped into two categories, namely load point and overall system indices. The calculation of both load point and overall system indices are necessary in order to obtain a complete picture of the overall system adequacy. A range of possible indices and the equipment 
outage models required to obtain these indices are also presented in this chapter. The differences in the indices resulting from using these three methods are also discussed in order to show the importance of using the ac load flow method in the calculation of adequacy indices for composite power systems.

It has been shown [81] that of the three approaches, the ac load flow method provides the most representative indices. The computation time, however, increases tremendously as the depth of contingency and the size of the system increases. Approximate methods can be utilized in order to reduce the computation time by limiting the number of contingencies that have to be investigated using the ac load flow method. Chapter 3 presents a series of adequacy indices evaluated using different ranking methods [41, 42]. The computation time and the differences in the adequacy indices compared to a reference case ac solution are presented. Three different performance indices are used to obtain the ranking lists. These lists can be prepared in two different ways and the advantages and disadvantages of these ranking lists are discussed in Chapter 3. The ranking cutoff criteria and the effects of a consecutive success cutoff criterion on computation time and the number of contingencies examined are also presented in Chapter 3. It is possible to employ an approximate selection method instead of using decoupled load flow for each of the contingencies. Efficient selection algorithms [82] for outages of generators, transmission lines and combinations of these elements are discussed in Chapter 3. The selection method can be used to examine both the continuity and the quality of power supply at major load centers for any type of contingency. The application of the method and its comparison with the reference case and ranking methods are illustrated using the two test systems in Chapter 3. It has been shown that $[41,42,82]$ the selection method provides more accurate results than the ranking method and therefore the selection method is utilized as the basic solution technique to consider security considerations in the reliability evaluation of composite systems.

The system operating state framework proposed in Reference [65] has been examined and extended in Chapter 4. An efficient approach which avoids the recursive use of the Sherman-Morrison correction formula for multiple lines outages is also 
presented. Only the line overload is considered as a system constraint in this chapter and a linear programming (LP) model for generation rescheduling and load curtailments and the solution techniques for the LP models are described. Both annual and annualized indices for the system operating states $[65,69]$ are presented and the effect of sorting the identical contingencies on the computation times are also presented.

The occurrence of contingencies, mainly transmission line outages, may result in voltage magnitude deviations from acceptable limits at some of the load buses in the system. Acceptable voltage levels at a bus, therefore, are important factors in reliability assessment. The effects of considering bus voltage magnitudes outside the acceptable limits to be a system problem are presented in Chapter 5. An LP model for voltage correction by changing the appropriate generation bus voltages and Q-load curtailment at different load buses was developed and is presented using a small test system. Annualized and annual indices considering only the voltage problem and the total constraint set defined in Chapter 4 are presented in Chapter 5.

The use of the Monte Carlo simulation method allows detailed modelling of precontingency conditions, generation and transmission outages and operating practices $[17,71]$. Parameters that define a system operating state, such as load and the state of generation and transmission components can be selected by random sampling and the sampled situations can be assigned to appropriate system operating states using the enumeration approach. The total sample probability or a fraction of the probability can be assigned to different states. Two hybrid approaches, considering simulation together with the enumeration technique, are discussed in Chapter 6 and used to calculate the operating states indices. The accuracy of a simulation approach to estimating a reliability index is related to the number of samples considered. The precision of estimation for the same number of samples or, conversely the number of samples for the same precision can be reduced using variance reduction techniques (VRT). Stratification after sampling and antithetic VRTs are considered and investigated in Chapter 6.

An electric power system planner is concerned both with the level of predicted reliability and the investment and operation alternatives associated with meeting the desired level. There are many alternatives that can be utilized for a system expansion 
plan to meet future load growth. A risk index designated as the Composite System Operating State Risk (CSOSR) has been defined in Chapter 7 from the probabilities of the normal and alert states. The utilization of the CSOSR for simple composite generation and transmission system expansion planning is discussed in Chapter 7 using the two test systems.

The load in a practical power system is changing continuously and therefore it is not economical to continuously run all the generating units required to satisfy the peak load. Units are usually added to or removed from service depending on the load levels. The generating units should be committed to service for different segments of a scheduling period in such a way that the operating cost is minimized with a satisfactory level of reliability. The unit commitment should be such that the commitment risk [2] is less than or equal to a specified level. In a composite generation and transmission system, the unit commitment should satisfy both HLI [83] and a composite system risk level. The time period used in an operating capacity evaluation is generally relatively small and known as the lead time [2]. The lead time is the time period for which no additional units can be brought into service. The lead time can vary from a few minutes to several hours depending on the type and size of the unit. The effect of unit commitment and the lead time on the indices of different operating states are considered in Chapter 8. The combination of unit commitment risk and the system operating state framework proposed in this thesis provides an important extension of conventional HLI analysis to the HLII domain. The total probability considered in a study can be increased for a given contingency depth using a capacity outage table instead of using the individual generating units. The utilization of capacity outage table to calculate the indices of different operating states is also demonstrated in this chapter.

The conclusions of the thesis are presented in Chapter 9. 


\section{ADEQUACY EVALUATION OF COMPOSITE POWER SYSTEMS - ENUMERATION APPROACH}

\subsection{INTRODUCTION}

Quantitative assessment of the adequacy of a bulk power system can be performed using a contingency enumeration approach $[47,68,76]$. The basic procedure involves the selection and evaluation of contingencies, the classification of each contingency according to selected failure criteria and the accumulation of adequacy indices. Various contingency enumeration techniques, depending upon the failure criteria and the intent behind the studies, are available in order to analyze the adequacy of a composite power system. The basic steps in an adequacy enumeration approach can be structured as shown in Figure 2.1. A contingency is tested under a specific base case condition in order to determine if the contingency causes any immediate system problem. If it does not cause any problem, a new contingency is taken and tested. The basic decisions

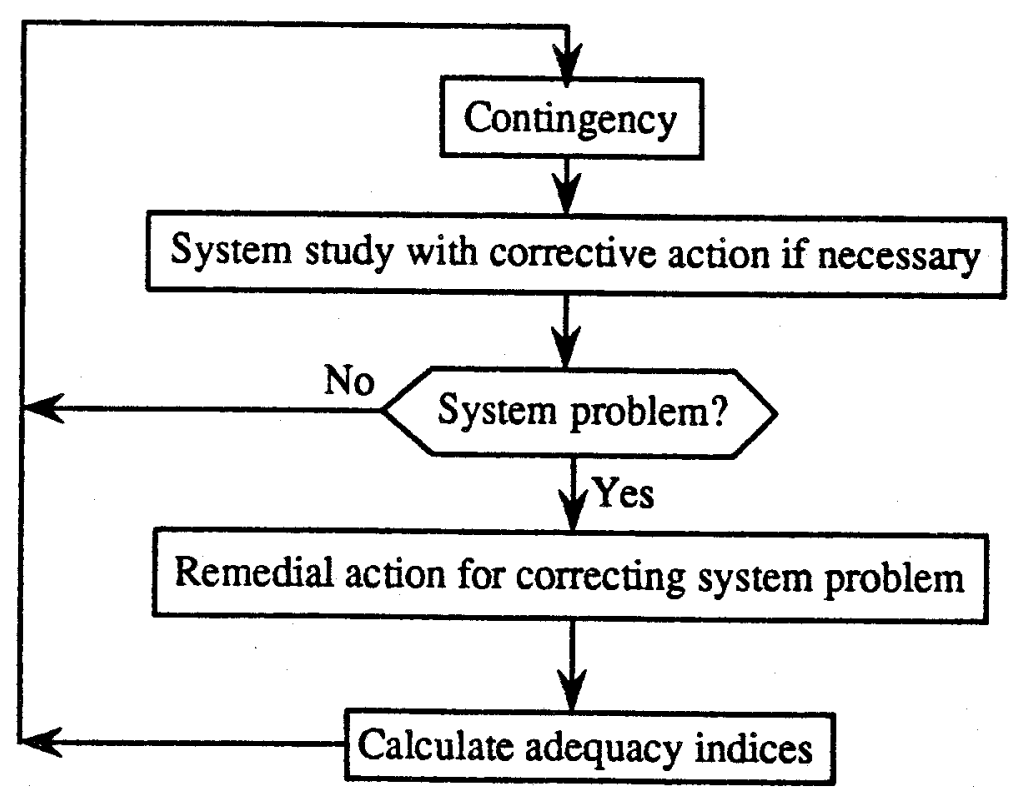

Figure 2.1: Contingency enumeration approach 
[76] which have to be made regarding the procedure to be used for a given adequacy study are:

1. selection of an appropriate network solution technique,

2. selection of an appropriate set of corrective actions,

3. selection of an appropriate contingency level for both generating unit and transmission line outages,

4. consideration of station originated and common cause outages and

5. calculation of an appropriate set of adequacy indices.

The adequacy assessment of a bulk power system generally involves the solution of the network configuration under selected outage situations. Various techniques, depending upon the adequacy criteria employed and the intent behind these studies, are available for use in analyzing the adequacy of a power system. The three basic analytical techniques are:

1. a network flow method $[77,84]$

2. dc load flow method $[47,78]$

3. ac load flow methods $[60,78,79]$

The selection of an appropriate technique is of prime importance and is an engineering decision. The key point is that the selected technique should be capable of satisfying the intent behind the studies from a management, planning and design point of view. The output from these studies may have to be related to consumer expectations, the standard of living and the economic and social consequences associated with an unreliable power supply.

It is not realistic to attempt to consider all possible contingencies in an adequacy evaluation study. The main constraint to considering a large number of outage events is the computation time required to solve these contingencies using an acceptable solution technique. In order to limit the number of contingencies, fixed criteria such as the selection of single or double level contingencies and/or variable criteria such as a frequency/probability cut-off limit and/or ranking cut-off limit etc. are presently utilized. The techniques used to assess the system have significant impacts on both the results 
obtained and the computation time required to achieve a solution. The computation time and solution accuracy for the network flow and the dc load flow methods are compared in this chapter with those of a reference ac load flow solution considering contingencies up to a certain level.

A selection of adequacy indices and the outage models $[2,85,86]$ required in the computation of adequacy indices are given in this chapter. The two test systems which are used throughout the remainder of the thesis to conduct various reliability studies are also presented.

\subsection{NETWORK SOLUTION TECHNIQUES}

The contingency enumeration approach structure shown in Figure 2.1 can be divided into the following two parts:

1. contingency evaluation and

2. adequacy index computation.

The contingency evaluation segment encompasses the following steps:

1. Modification of the configuration due to the outage of various components.

2. Determination of system problems using an appropriate method depending on the failure criteria

3. Implementation of corrective action, if necessary, such as rescheduling of the generating units, line overload alleviation, correction of bus voltages and load curtailment at buses, etc..

It can easily be appreciated that the contingency enumeration approach will require a large number of network solutions if it is to consider all generator and line outage conditions. This calls for fast solution techniques using simplified or approximate methods. Various techniques, depending upon the adequacy criteria employed and the intent behind the studies are available in order to analyze the adequacy of a power system. One of the simplest approaches is to treat the system as a transportation model [77] and to examine it in terms of its ability to ensure the continuity of power supply at various load centers. Approximate load flow techniques, such as dc load flow etc., are quite simple and fast but only provide an estimate of the line power flows, without 
including any estimate of the bus voltages and the reactive power limits of the generating units, etc.. If the quality of the power supply (proper voltage levels and correct MVAr limits of the generating units) is an important adequacy criterion, then more accurate ac load flow methods $[78,79]$ such as Gauss-Seidel, Newton-Raphson and second order load flow techniques must be employed in order to calculate the adequacy indices. These techniques are not often used because they are computationally more expensive and have large storage requirements. Several computationally faster ac load flow techniques which are modifications of the Newton-Raphson load flow approach are available. The fast decoupled load flow technique is one of these methods, The following is a brief summary of the network solution techniques studied in this thesis.

\subsubsection{Network Flow Method}

The linear network flow method or transportation network model is the simplest network solution technique in which the only concern is the movement of a particular commodity from a number of sources to a number of demand centers. The network flow method can be used in composite power system studies with some approximations. This method, when applied in composite system reliability evaluation studies, is basically concerned with the continuity of the power flow from the generating buses (sources) to the major load buses (sinks) in order to supply the load demand in the system. The constraints are the generating capacity available at the generating bus and the power carrying capacities of the transmission lines. The network flow model solution can be formulated either as a maximal flow problem or as a linear programming problem [77]. The algorithm employed in the studies presented in this thesis is based on the concepts of maximal flow or minimal cut. The mathematical formulation of the method can be given by considering a power network $\mathrm{G}=[\mathrm{N} ; \mathrm{A}]$ consisting of one generating bus (source) $\mathrm{s}$ and one load bus (sink) $t$ as [84]

Maximize F subject to the following two constraints

$$
\sum_{j \in A(i)} f(i, j)-\sum_{j \in B(i)} f(j, i)=\left\{\begin{array}{cl}
F, & i=s \in N \\
0, & i \neq s, t \\
-F, & i=t \in N
\end{array}\right.
$$


and

$$
f(i, j) \leq c(i, j) \quad \text { for all }(i, j) \in A
$$

where:

$$
\begin{aligned}
& A \quad=a \text { set of arcs (transmission lines and/or transformer) } \\
& N \quad=\text { a set of nodes (buses) } \\
& c(i, j) \quad=\text { maximum load carrying capacity of the arc between nodes } i \text { and } j \\
& A(i) \quad=(j \in N \mid(i, j) \in A) \\
& B(i) \quad=(j \in N \mid(i, j) \in A) \text { and } \\
& F \quad=\text { load flow from s to } t .
\end{aligned}
$$

Equation 2.1 states that the net flow out of the node $i$ is $F$ and that net flow out of node $j$ is $-\mathrm{F}$, whereas the net flow out of any intermediate node is zero. Equation 2.2 limits the flow in the arcs to the maximum permissible capacity of the arcs.

In a practical power network,there is more than one source and more than one sink. The problem can be converted to the simple ( $s$ to $t$ ) maximum flow problem by adding a new artificial source $s$ and sink $t$ with added arcs leading from $s$ to all $n_{s}$ sources (generating buses) and from every sink (load buses) to $t$ [84]. The network flow model preserves only the power balance at each node of the network and does not satisfy Kirchhoff's voltage law. It's application to an electrical network therefore, is an approximation which may not be accurate for meshed systems. It has been, however, applied in composite power system planning problems [87] because of its simplicity.

\subsubsection{Load Flow Method}

One of the simplest network solution techniques applied in contingencies studies is the dc load flow. This approach uses the following linear model:

$$
[\mathrm{P}]=[\mathrm{B}][\delta]
$$

where:

[P] = vector of bus power injection, 
[B] = system susceptance matrix and

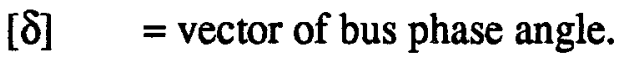

The dimensions of $[\mathrm{B}]$ and $[\mathrm{P}]$ are $(\mathrm{N}-1 \mathrm{XN}-1)$ and $(\mathrm{N}-1 \mathrm{X} 1)$ respectively, where $\mathrm{N}$ is the number of buses in the system, as one bus is specified as the "slack" or "swing" bus.

The vector of bus phase angle [ $\delta$ ] can be obtained by solving Equation 2.3 using [B] and [P]. Optimal ordering and triangular factorization of the system susceptance matrix are used to achieve rapid solution time. The bus phase angles, computed using forward and backward substitution, are then used to determine the individual branch flows given by:

$$
\mathrm{P}_{p q}=\frac{\delta_{p}-\delta_{q}}{\mathrm{X}_{p q}}
$$

where:

$\mathrm{P}_{p q} \quad=$ real power flow from bus $\mathrm{p}$ to bus $\mathrm{q}$,

$\delta_{p} \quad=$ phase angle at bus $\mathrm{p}$,

$\delta_{q} \quad=$ phase angle at bus $\mathrm{q}$ and

$\mathrm{X}_{p q} \quad=$ reactance of the line between bus $\mathrm{p}$ and bus $\mathrm{q}$.

It can be seen from Equation 2.3 that for a fixed set of power injections [P], if a line or

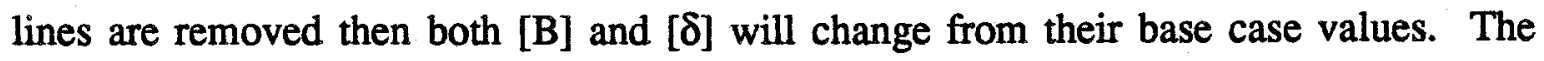
changes in angle vector can be computed using the Sherman-Morrison correction formula [80] instead of rebuilding and refactorizing the system susceptance matrix [B]. The new line flows can be calculated from Equation 2.4 using the new values of $[\delta]_{\text {new }}$.

Voltage/VAR effects and system losses are not considered. The solution is fast and free of convergence problems. 


\subsubsection{Fast Decoupled Load Flow Method}

The fast decoupled load flow technique is a good compromise between ac and dc load flow approaches in regard to storage requirements and solution speed. It can also be used to check the continuity as well as the quality of a power system thus meeting the two important adequacy requirements. Reference [75] presents work on composite generation and transmission system adequacy which utilizes the fast decoupled load flow algorithm developed by Stott and Alsac [60]. A brief description of the fast decoupled load flow technique is given below.

The general equations for the power mismatch for all system buses except the swing bus are as follows

$$
\left[\begin{array}{l}
\Delta \mathrm{P} \\
\Delta \mathrm{Q}
\end{array}\right]=\left[\begin{array}{ll}
\mathrm{J} 1 & \mathrm{~J} 2 \\
\mathrm{~J} 3 & \mathrm{~J} 4
\end{array}\right]\left[\begin{array}{l}
\Delta \delta \\
\Delta \mathrm{V} / \mathrm{V}
\end{array}\right] .
$$

The terms of the Jacobian matrix are the partial derivatives of $P$ and $Q$ with respect to $V$ and $\delta$.

The decoupled load flow algorithms neglect the weak coupling between changes in real power and voltage magnitude, and changes in reactive power and voltage phase angles. Neglecting the weak couplings noted above, Equation 2.5 becomes

$$
\begin{aligned}
{\left[\begin{array}{c}
\Delta \mathrm{P} \\
\Delta \mathrm{Q}
\end{array}\right] } & =\left[\begin{array}{ll}
\mathrm{J} 1 & 0 \\
0 & \mathrm{~J} 4
\end{array}\right]\left[\begin{array}{l}
\Delta \delta \\
\Delta \mathrm{V} / \mathrm{V}
\end{array}\right] \\
\text { or, }[\Delta \mathrm{P}] & =[\mathrm{J} 1][\Delta \delta] \text { and } \\
{[\Delta \mathrm{Q}] } & =[\mathrm{J} 4][\Delta \mathrm{V} / \mathrm{V}]
\end{aligned}
$$

where:

$$
\begin{aligned}
& \Delta \mathrm{P}_{p} \quad=\text { Active power mismatch at bus } \mathrm{p}, \\
& \Delta \mathrm{Q}_{p} \quad=\text { Reactive power mismatch at bus } \mathrm{p}, \\
& \Delta \delta_{p} \quad=\text { Increment in the phase angle of the voltage at bus } \mathrm{p}, \\
& \Delta \mathrm{V}_{p} \quad=\text { Increment in the magnitude of the voltage at bus } \mathrm{p},
\end{aligned}
$$


J's = Submatrices of the Jacobian matrix,

$\delta_{p} \quad=$ Phase-angle of the voltage at bus $\mathrm{p}$ and

$\mathrm{V}_{p} \quad=$ Magnitude of the voltage at bus $\mathrm{p}$.

Equations 2.6 and 2.7 can be further simplified by making the following assumptions, which are usually valid in a practical power system:

$$
\begin{aligned}
& \cos \left(\delta_{p}-\delta_{q}\right) \approx 1.0, \\
& \mathrm{~g}_{p q} \cdot \sin \left(\delta_{p}-\delta_{q}\right) \ll<\mathrm{b}_{p q} \text { and } \\
& \mathrm{Q}_{p} \ll \mathrm{b}_{p p} \cdot \mathrm{v}_{p}^{2}
\end{aligned}
$$

where:

$$
\begin{array}{ll}
\mathrm{g}_{p q}-\mathrm{jb} \mathrm{b}_{p q} & =\begin{array}{l}
\text { series admittance of the line connecting the buses } \\
\mathrm{p} \text { and } \mathrm{q} \text { and }
\end{array} \\
\mathrm{Q}_{p} & =\text { reactive power at bus } \mathrm{p} .
\end{array}
$$

Using these assumptions, Equations 2.6 and 2.7 can be rewritten as follows:

$$
\begin{aligned}
& {[\Delta \mathrm{P}]=\left[\mathrm{V} \cdot \mathrm{B}^{\prime} . \mathrm{V}\right][\Delta \delta]} \\
& {[\Delta \mathrm{Q}]=\left[\mathrm{V} \cdot \mathrm{B}^{\prime \prime} . \mathrm{V}\right][\Delta \mathrm{V} / \mathrm{V}] .}
\end{aligned}
$$

The final equations after making further physically-justifiable simplifications [60] are given below.

$$
\begin{aligned}
& {[\Delta \mathrm{P} / \mathrm{V}]=\left[\mathrm{B}^{\prime}\right][\Delta \delta]} \\
& {[\Delta \mathrm{Q} / \mathrm{V}]=\left[\mathrm{B}^{\prime \prime}\right][\Delta \mathrm{V}]}
\end{aligned}
$$

Both matrices $\left[\mathrm{B}^{\prime}\right]$ and $\left[\mathrm{B}^{\prime \prime}\right]$ are real, sparse and contain only network admittances. Since $\left[B^{\prime}\right]$ and $[B "]$ are constant, they need to be inverted or factorized only once at the beginning of the iterative process. The magnitude of the voltage at each load bus and the voltage phase angle at each bus except the swing bus are modified as given below: 


$$
\begin{aligned}
& {[\delta]_{\text {new }}=[\delta]_{\text {old }}+[\Delta \delta]} \\
& {[\mathrm{V}]_{\text {new }}=[\mathrm{V}]_{\text {old }}+[\Delta \mathrm{V}]}
\end{aligned}
$$

Power mismatch $[\Delta \mathrm{P}]$ and $[\Delta \mathrm{Q}]$ are calculated for these new values of bus voltage and bus angle. Equations 2.8 and 2.9 are iterated in some defined manner towards an exact solution i.e. when power mismatches are less than the tolerances. In the case of line and/or transformer outages, the Sherman-Morrison correction formula can be used to reflect the outages instead of rebuilding and refactorizing the system matrices [B'] and [B"].

\subsection{SHERMAN-MORRISON CORRECTION}

The Sherman-Morrison correction formula can be used to obtain load flow solutions under circuit outage conditions. Instead of rebuilding and refactorizing the system admittance matrices for each line contingency, a simple correction formula is used to adjust the base solution to effectively represent the line outage. The algorithm is applicable to both the dc and the decoupled load flow techniques.

Essentially, the correction factors can be computed by one forward and backward substitution using the original factorized system admittance matrix and the base solution vector. Multiple line outages can be represented by applying the formula recursively, and updating the solution vector at each step.

\section{Application to the de load flow :}

The base case dc load flow solution can be represented by the equation

$$
\begin{aligned}
{[\mathrm{P}] } & =[\mathrm{B}][\delta] \\
\text { or, } \quad[\delta] & =[\mathrm{B}]^{-1}[\mathrm{P}]
\end{aligned}
$$

where all the quantities of Equation 2.10 are already defined. The outage of line ' $l$ ' connecting buses ' $p$ ' and ' $q$ ' can be reflected in [B] by

$$
[\mathrm{B}]_{\text {new }}=[\mathrm{B}]+\mathrm{bmm}^{t}
$$


where $\mathrm{b}=-\left(1.0 / \mathrm{X}_{p q}\right)$ and $\mathrm{m}$ is a column vector with all elements zero except element ' $\mathrm{p}$ ' which is +1 and element ' $q$ ' which is -1 . Applying the SM correction formula,

$$
\begin{aligned}
{[\mathrm{B}]^{-1}{ }_{\text {new }} } & =[\mathrm{B}]^{-1}-\mathrm{c}[\mathrm{B}]^{-1} \mathrm{~mm}^{t}[\mathrm{~B}]^{-1} \\
& =[\mathrm{B}]^{-1}-\mathrm{c}[\mathrm{Z}] \mathrm{m}^{t}[\mathrm{~B}]^{-1}
\end{aligned}
$$

where:

$$
\begin{aligned}
{[\mathrm{Z}] } & =[\mathrm{B}]^{-1} \mathrm{~m} \text { and } \\
\mathrm{c} & =\left\{1 / \mathrm{b}+\mathrm{m}^{t}[\mathrm{~B}]^{-1} \mathrm{~m}\right\}^{-1} \\
& =\left\{1 / \mathrm{b}+\mathrm{Z}_{p}-\mathrm{Z}_{q}\right\}^{-1} .
\end{aligned}
$$

The solution to the outage problem, from Equation 2.10 is

$$
\begin{aligned}
{[\delta]_{\text {new }} } & =\left\{[\mathrm{B}]^{-1}-\mathrm{c}[\mathrm{Z}] \mathrm{m}^{t}[\mathrm{~B}]^{-1}\right\}[\mathrm{P}] \\
& =[\delta]-c[\mathrm{Z}] \mathrm{m}^{t}[\mathrm{~B}]^{-1}[\mathrm{P}] \\
& =[\delta]-c\left(\delta_{p}-\delta_{q}\right)[\mathrm{Z}] \\
& =[\delta]+[\Delta \delta] .
\end{aligned}
$$

Equation 2.13 can be duplicated recursively for multiple outages:

$$
\begin{aligned}
& {\left[\delta_{1}\right]=\left[\delta_{0}\right]-c_{1}[\mathrm{Z} 1] \mathrm{m}_{1}{ }^{t}\left[\delta_{0}\right]} \\
& {\left[\delta_{2}\right]=\left[\delta_{1}\right]-c_{2}[\mathrm{Z} 2] \mathrm{m}_{2}{ }^{t}\left[\delta_{1}\right]} \\
& {\left[\delta_{3}\right]=\left[\delta_{2}\right]-c_{3}[\mathrm{Z} 3] \mathrm{m}_{3}{ }^{t}\left[\delta_{2}\right]}
\end{aligned}
$$

where, the subscripts $0,1,2,3$ represent the base case, single contingency, double contingency and triple contingency respectively and

$$
\begin{aligned}
{[\mathrm{Z} 1] } & =[\mathrm{B}]^{-1} \mathrm{~m}_{1} \\
{[\mathrm{Z} 2] } & =[\mathrm{B}]^{-1} \mathrm{~m}_{2}-\mathrm{c}_{1}[\mathrm{Z} 1] \mathrm{m}_{1}^{t}[\mathrm{~B}]^{-1} \mathrm{~m}_{2} \\
& =\left(\mathrm{I}-\mathrm{c}_{1}[\mathrm{Z} 1] \mathrm{m}_{1}^{t}\right)[\mathrm{B}]^{-1} \mathrm{~m}_{2} \\
\mathrm{c}_{2} & =1.0 /\left(1 / \mathrm{b}_{2}+\mathrm{m}_{2}^{t}[\mathrm{Z} 2]\right) \\
{[\mathrm{Z} 3] } & =\left(\mathrm{I}-\mathrm{c}_{2}[\mathrm{Z} 2] \mathrm{m}_{2}^{t}\right) \cdot\left(\mathrm{I}-\mathrm{c}_{1}[\mathrm{Z} 1] \mathrm{m}_{1}^{t}\right)[\mathrm{B}]^{-1} \mathrm{~m}_{3}
\end{aligned}
$$




$$
c_{3}=1.0 /\left(1 / b_{3}+m_{3}{ }^{t}[Z 3]\right)
$$

\section{Application to the decoupled load flow :}

Equation 2.8 or 2.9 can be represented in the base case problem as follows.

$$
[R]=\left[\mathrm{B}_{0}\right]\left[\mathrm{E}_{0}\right]
$$

The outage of line ' $l$ ' connecting bus ' $p$ ' and ' $q$ ' can be given by Equation 2.11 as

$$
\left[\mathrm{B}_{1}\right]=\left[\mathrm{B}_{0}\right]+b \mathrm{~mm}^{\mathfrak{l}} \text {. }
$$

Depending on the types of the connected buses, only one row, ' $p$ ' or ' $q$ ' might be present in [ $\left.B^{\prime}\right]$, in which case either $m_{p}$ or $m_{q}$ is zero. If both the connected buses are PV or if one is a PV bus and the other is a slack bus, then $\left[\mathrm{B}^{\prime \prime}\right]$ requires no modification. The iterative process is repeated using the original lower and upper triangular (LU) factors of $\left[\mathrm{B}_{0}\right]$ with a correction for the solution vector $\left[\mathrm{E}_{0}\right]$ ( i.e. voltage or phase angle ) at the end of each iteration obtained using Equation 2.15. The equation in this case is

$$
\left[\mathrm{E}_{1}\right]=\left[\mathrm{E}_{0}\right]-\mathrm{c}[\mathrm{Z}] \mathrm{m}^{t}\left[\mathrm{E}_{0}\right] \text {. }
$$

The above process can be applied recursively for multiple simultaneous outages. The solution $[E]$ is corrected successively as the effect of each line outage is introduced in a similar manner to that described for [ $\delta]$ by the set of Equations 2.15 and 2.16. The SM correction formula is applied for line outage contingency evaluation in order to save computation time with both dc and decoupled ac load flow in Chapters 2 and 3 of this thesis.

\subsection{STATE-SPACE MODELS}

In order to compute probability, frequency and duration measures of system reliability, component models are normally presented using state-transition diagrams. Single and multiple component models involving both independent and dependant failures are illustrated in the following sections $[30,88]$. 


\subsubsection{A Single Component Model}

The state transition diagram for a single component is shown in Figure 2.2. The

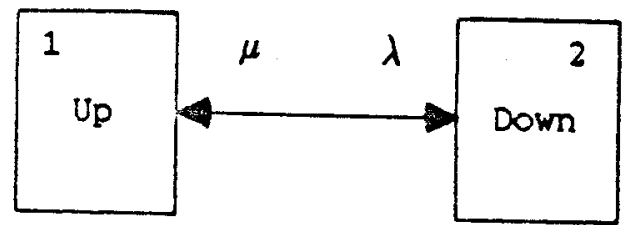

$$
\begin{aligned}
& \lambda=\text { Failure rate } \quad\left[\text { year }^{-1}\right] \\
& \mu=\text { Repair rate } \quad\left[\text { year }^{-1}\right. \text { ] } \\
& \mathrm{m}=\frac{1}{\lambda}=\text { Mean in-service duration } \quad \text { [years] } \\
& \mathrm{r}=\frac{1}{\mu}=\text { Mean outage duration [years] }
\end{aligned}
$$

Figure 2.2: Single component two state model

probability, availability, unavailability and frequency measures for this model can be derived as follows:

Availability,

$$
A=\frac{m}{m+r}=\frac{\mu}{\mu+\lambda}=P_{1}
$$

$=$ Probability of the component being in state 1 .

Unavailability,

$$
U=\frac{r}{m+r}=\frac{\lambda}{\mu+\lambda}=P_{2}
$$

$=$ Probability of the component being in state 2 .

$$
\begin{aligned}
A+U= & 1 \\
f= & A . \lambda \\
& =U . \mu \\
& =\text { Frequency of transfer from the in-service to the } \\
& \text { outage state (failure frequency) } \\
& =\text { Frequency of transfer from the outage to the in- } \\
& \text { service state ( restoration frequency ). }
\end{aligned}
$$


The basic outage data required to support the single component model are the failure rate $\lambda$ and the repair rate $\mu$.

\subsection{Model For Independent Overlapping Outages}

The model for independent overlapping outages assumes that the occurrence of one outage does not affect the probability of occurrence of other outages. The model also assumes that the outage duration of one component is not affected by whether or not other components are in service or on outage. The state transition diagram for two independent overlapping outages is given in Figure 2.3. The probabilities for each of the

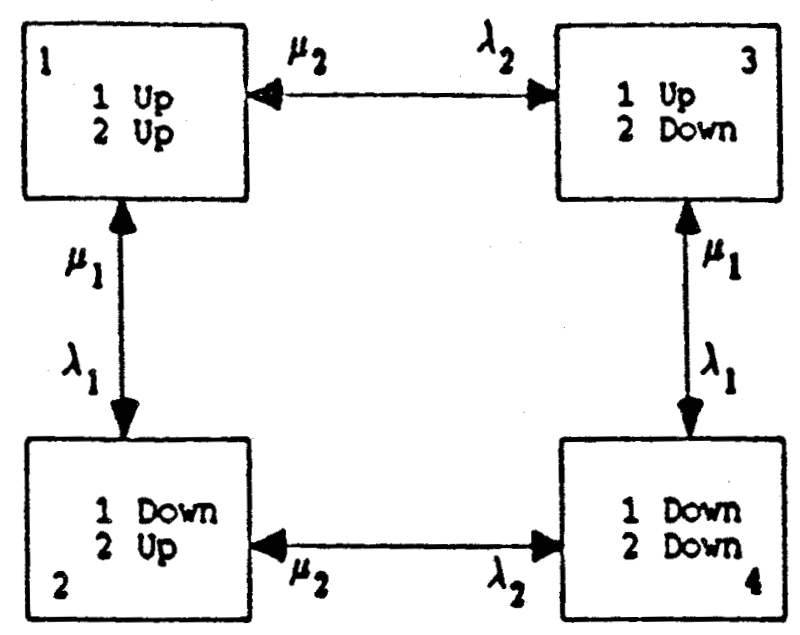

Figure 2.3: Model for two independent overlapping outages

four states are:

$$
\begin{aligned}
& P_{1}=A_{1} A_{2}=\frac{\mu_{1} \mu_{2}}{D} \quad \text { Probability of both components up } \\
& P_{2}=U_{1} A_{2}=\frac{\lambda_{1} \mu_{2}}{D} \\
& \left.P_{3}=A_{1} U_{2}=\frac{\mu_{1} \lambda_{2}}{D}\right\} \begin{array}{l}
\text { Probability of one component up and } \\
\text { one component down }
\end{array} \\
& P_{4}=U_{1} U_{2}=\frac{\lambda_{1} \lambda_{2}}{D} \quad \text { Probability of both components down }
\end{aligned}
$$

where, $D=\left(\lambda_{1}+\mu_{1}\right)\left(\lambda_{2}+\mu_{2}\right)$ and $A_{i}$ and $U_{i}$ are the availability and unavailability of the $i^{\text {th }}$ component. 
The frequencies and durations for the different states are:

$$
\begin{array}{ll}
f_{1}=P_{1}\left(\lambda_{1}+\lambda_{2}\right) & r_{1}=\frac{P_{1}}{f_{1}}=\frac{1}{\left(\lambda_{1}+\lambda_{2}\right)} \\
f_{2}=P_{2}\left(\mu_{1}+\lambda_{2}\right) & r_{2}=\frac{P_{2}}{f_{2}}=\frac{1}{\left(\mu_{1}+\lambda_{2}\right)} \\
f_{3}=P_{3}\left(\lambda_{1}+\mu_{2}\right) & r_{3}=\frac{P_{3}}{f_{3}}=\frac{1}{\left(\lambda_{1}+\mu_{2}\right)} \\
f_{4}=P_{4}\left(\mu_{1}+\mu_{2}\right) & r_{4}=\frac{P_{4}}{f_{4}}=\frac{1}{\left(\mu_{1}+\mu_{2}\right)}
\end{array}
$$

This type of model can be extended to three or more components on independent overlapping outage. The state transition diagram becomes complex for three or more independent overlapping outages but the equations for the state probabilities, frequencies and durations can be generalized from the two component case.

The severity associated with an outage event increases as the depth of contingency increases. It is, therefore, necessary to probe deeper levels in search of more severe load/energy curtailment situations. The number of possible independent outage events increases significantly as the contingency depth increases. The computation time, therefore, increases considerably when high level independent outages are considered using an ac load load flow method in the contingency enumeration approach. The computation time, however, does not increase very much with the addition of common cause outages and station originated outages. One basic concern in this research was to achieve a reduction in computation time by reducing the number of independent outages to be considered when using an ac load flow method. Only independent outages are therefore, considered in this thesis in order to assess the speed and accuracy of different approximate methods. Common cause and station originated outages are not considered in these studies. The contribution to the adequacy indices from these outage events are very significant but it is necessary to examine these outage events using an accurate method rather than using an approximate method as in the case of independent outages. The effect of these outages, however, can be considered using the approximate methods without a significant increase in computation time by modifying the probabilities and frequencies of the appropriate states. 


\subsection{CORRECTIVE ACTIONS}

The occurrence of a system problem may by itself be recorded as a failure event. In many cases, however, it may be possible to eliminate a system problem by taking appropriate corrective action. It is, therefore, of interest to determine whether it is possible to eliminate a system problem by employing proper corrective actions. There is no consensus among power utilities and related organizations regarding uniform failure criteria and therefore all organizations do not use the same fundamental solution technique to calculate the adequacy of their system [89]. The basic justification for these differences lies in the intent behind the adequacy studies. The experience of one organization with those factors that contribute to their system failure may vary greatly with respect to that of other organizations. Factors such as the meteorological conditions in the region, the configuration of the network, the protection schemes employed, the system generation and load composition, and the dependent and independent probability of component failure can assume different degrees of importance in different systems. Attention is, therefore, primarily focused on the adequacy evaluation of those outage contingencies which apparently seem to result in failure. The validity of a particular approach may be quite justified for a particular system but may not provide suitable estimates of the adequacy of other systems. It may not, therefore, be possible to develop a general purpose approach because of the basic differences between power systems. It is, however, necessary to recognize consistent failure criteria for each approach. Table 2.1 shows recommended failure criteria for the three solution techniques described earlier. Failure criteria of either the network flow method or the dc load flow method are a subset of the failure criteria of ac load flow methods.

On the basis of the failure criteria, the broad categories of corrective actions $[60,73,75,89,90]$ that can be employed are as follows:

1. Generation rescheduling in the case of capacity deficiency in the system.

2. Correction of a generating unit MVAr limits violations.

3. Bus isolation and system splitting under transmission line(s)/ transformer(s) outages.

4. Alleviation of line overloads [75, 90].

5. Correction of voltage problem at a bus and the solution of ill-conditioned network situations when using ac load flow methods. 
Table 2.1: Recommended failure criterion for different solution techniques

Network Flow
Method $\quad\left\{\begin{array}{l}\text { 1. } \begin{array}{l}\text { Load curtailment at bus(es) due to capacity deficiency } \\ \text { in the system }\end{array} \\ \text { 2C Load } \\ \text { Flow Method } \\ \text { 3. Load curtailment, if necessary, at isolated bus(es) } \\ \text { Load curtailment, if necessary, at bus(es) in the network } \\ \text { islands formed due to line outages. } \\ \text { 4. } \begin{array}{l}\text { Load curtailment at bus(es) due to line/transformer } \\ \text { overloads. }\end{array} \\ \text { AC Load }\end{array}\right] \begin{aligned} & \text { 5. Voltage collapse at system bus(es). } \\ & \text { 6. Generating unit MVAr limits violations. } \\ & \text { 7. } \text { Ill-conditioned network situations. }\end{aligned}$

6. Load curtailment in the event of a system problem.

The selection of a particular corrective action is dependent upon the situation that causes a failure in the network. A description of the corrective actions and load curtailment procedures can be found in References [70,93]

\subsection{ADEQUACY INDICES}

There are three fundamental parameters in the calculation of system adequacy indices. These parameters are:

1. Frequency of events.

2. Duration of events.

3. Severity of events.

Event probability can be derived from the event frequency and duration using the relation Probability $=$ Frequency $\times$ Duration

Computationally, it is often easiest to compute the event probabilities and frequencies from which the event durations can be derived. The severity of an event depends on the components under outage, their relative importance and their location in the network configuration. An outage event may affect a wide area of the system or it may affect 
only a small group of buses or perhaps a single bus. The adequacy indices should focus attention on those portions of the system that are directly affected by the outage event. The total contribution of all possible outage contingencies should indicate those areas in the system which have low reliability and are prone to disturbances. Overall system indices can not provide this information. It is therefore not desirable to draw conclusions regarding the adequacy of a particular system load point from overall system indices or bus average indices. Adequacy indices can be divided into two categories according to the way the severity of outage events are measured. The two categories can be designated as

1. Load point indices.

2. System indices.

The need for determining individual load point indices is also necessitated by the fact that the effect of high level outage events is not uniformly distributed over the entire system. First level and second level contingencies may be sufficient to provide adequacy indices with a reasonable accuracy at some system buses. At other buses, higher level contingencies must be considered before any significant problem is experienced.

In a similar manner, varying the load at each bus of a power system in equal proportion may not result in a proportionate variation of the indices at each bus. This is due to the fact that load flow studies involve the solution of non-linear simultaneous equations. The effect of load variation may not therefore be uniform at each bus, depending upon the network configuration and the system component parameters.

In the studies shown in this thesis, the main criteria selected for defining unacceptable quality of power supply at a load point are:

1. The load point voltage being less or greater than a specified minimum or maximum value respectively and/or

2. The inability of the system to supply the load connected to that bus without line overloads.

A comprehensive list of the basic indices $[2,4,70,85]$ considered is as follows. These indices are usually calculated on an annual basis. Other indices such as 'maximum 
values' and 'average values' for both load point and system indices are described in detail in Reference [2].

\subsubsection{Load Point Indices}

\section{Basic Values :}

$$
\begin{aligned}
& \text { Probability of failure }=\sum_{j} P_{j} P_{k j} \\
& \text { Frequency of failure }=\sum_{j} F_{j} P_{k j}
\end{aligned}
$$

where:

$j$ is an outage condition in the network.

$P_{j}$ is the state probability of the outage event $j$.

$F_{j}$ is the frequency of occurrence of the outage event $j$.

$P_{k j}$ is the probability of load at bus $k$ exceeding the maximum load that can be supplied at that bus during the outage event $j$. For a fixed load level considered for a specific period of time, $P_{k j}$ will be equal to zero if the total load at bus $k$ can be supplied without any problem but $P_{k j}$ will be unity if there is some problem in supplying the total load at bus $k$.

$$
\text { Expected number of voltage violations }=\sum_{j \varepsilon v} F_{j}
$$

where $j \varepsilon v$ includes all contingencies which cause voltage violation at bus $k$.

$$
\text { Expected number of load curtailments }=\sum_{j \varepsilon x, y} F_{j}
$$

where $j \varepsilon x$ includes all contingencies resulting in load curtailment at bus $k$ and $j \varepsilon y$ includes all contingencies which result in an isolation of bus $k$.

$$
\text { Expected load curtailed }=\sum_{j \in x, y} L_{k j} F_{j}(\mathrm{MW})
$$

where $L_{k j}$ is the load curtailment in MW at bus $k$ to alleviate line overloads arising due to the outage event $j$, or load not supplied at an isolated bus $k$ due to the outage event $j$. 
Expected energy not supplied

$$
\begin{aligned}
& =\sum_{j \varepsilon x, y} L_{k j} D_{k j} F_{j} \quad(\mathrm{MWh}) \\
& =\sum_{j \varepsilon x, y} L_{k j} P_{j} 8760.0 \quad(\mathrm{MWh})
\end{aligned}
$$

where $D_{k j}$ is the duration in hours of the load curtailment arising due to the outage event $j$; or the duration in hours of the load curtailment at an isolated bus $k$ due to the outage event $j$.

Expected duration of load curtailment

$$
\begin{aligned}
& =\sum_{j \in x, y} D_{k j} F_{j} \quad \text { (hours) } \\
& =\sum_{j \in x, y} P_{j} 8760.0 \text { (hours) }
\end{aligned}
$$

\subsubsection{System Indices}

Basic values :

Bulk Power Supply Disturbances (BPSD) $=\sum_{k} \sum_{j \varepsilon x, y} F_{j}$

Bulk Power Interruption Index (BPII)

$$
=\frac{\sum_{k} \sum_{j \varepsilon x, y} L_{k j} F_{j}}{L_{s}} \quad \text { (MW/MW-Year) }
$$

Bulk Power Supply Average MW Curtailment (BPSAMC)

$$
=\frac{\sum_{k} \sum_{j \varepsilon x, y} L_{k j} F_{j}}{\sum_{j \varepsilon x, y} F_{j}} \quad \text { (MW/disturbance) }
$$


Bulk Power Energy Curtailment Index (BPECI).

$$
=\frac{\sum_{k} \sum_{j \varepsilon x, y} 60.0 L_{k j} D_{k j} F_{j}}{L_{s}} \text { (system minutes) }
$$

This is also called 'Severity Index'.

Modified Bulk Power Energy Curtailment Index (MBPECI)

$$
=\frac{\sum_{k} \sum_{j \varepsilon x, y} L_{k j} D_{k j} F_{j}}{L_{s} 8760.0}
$$

where $L_{s}$ is the total system load.

These indices when calculated for a single fixed load level over a period of one year are referred to as "annualized indices". In practical systems, the load does not remain constant throughout the period and the effect of a variable load level can be included in order to produce more representative "annual indices".

If the values of any index are $x_{1}, x_{2}, x_{3}, \ldots, x_{n}$ for load levels $l_{1}, l_{2}, l_{3}, \ldots, l_{n}$ respectively and the probability of occurrence of the load levels $l_{1}, l_{2}, l_{3}, \ldots, l_{n}$ are $p_{1}, p_{2}$, $p_{3}, \ldots, p_{n}$ respectively, then the annual index $\bar{x}$ is

$$
\bar{x}=\left(p_{1} x_{1}+p_{2} x_{2}+\ldots+p_{n} x_{n}\right)
$$

The basic and average values for the annual indices will be different from the annualized values obtained using the peak load levels. The maximum indices remain unchanged as these represent the maximum value of an index for any load level over the period of study.

Indices such as the total number of voltage violation contingencies, total number of load curtailment contingencies, total number of firm load curtailment contingencies, total number of non-convergent contingencies can also be calculated in addition to the indices shown in Equations 2.22 to 2.33. 


\subsection{TEST SYSTEMS}

Adequacy evaluation studies have been conducted in this thesis using the following two test systems:

1. the Roy Billinton Test System [91] and

2. the IEEE Reliability Test System [38].

The following is a brief description of these systems:

\subsubsection{The Roy Billinton Test System (RBTS)}

This is a small composite power system which can be used to conduct a large number of adequacy studies with low computation time. This system has been developed by Professor Roy Billinton and is utilized extensively in the reliability research work conducted at the University of Saskatchewan. The single line diagram of this system is shown in Figure 2.4. The bus data, the line data and the generator data of this system are given in Appendix A. The system has 2 generator (PV) buses, 4 load (PQ) buses, 9 lines and 11 generating units. The minimum and maximum ratings of the generating units for this system are $5 \mathrm{MW}$ and $40 \mathrm{MW}$ respectively. The total system generation is 240 MW and the total system load is $185 \mathrm{MW}$. The voltage limits for this system are assumed to be 1.05 and 0.97 p.u.

\subsubsection{The IEEE Reliability Test System (IEEE-RTS)}

The single line diagram of this system is shown in Figure 2.5. This 24 bus system was established by an IEEE Task Force in 1979. This test system is relatively large and is used extensively as a reference network to test and develop different methods for adequacy evaluation. The bus data, the line data and the generator data of this system are given in Appendix B. This system has 10 generator (PV) buses, 10 load (PQ) buses, 33 transmission lines and 5 transformers. The total number of generating units is 32 . The minimum and maximum rating of the generating units are $12 \mathrm{MW}$ and $400 \mathrm{MW}$ respectively. The total system generation is $3405 \mathrm{MW}$ and total system load is $2850 \mathrm{MW}$. The voltage limits for the system buses are assumed to be 1.05 and 0.95 p.u. 


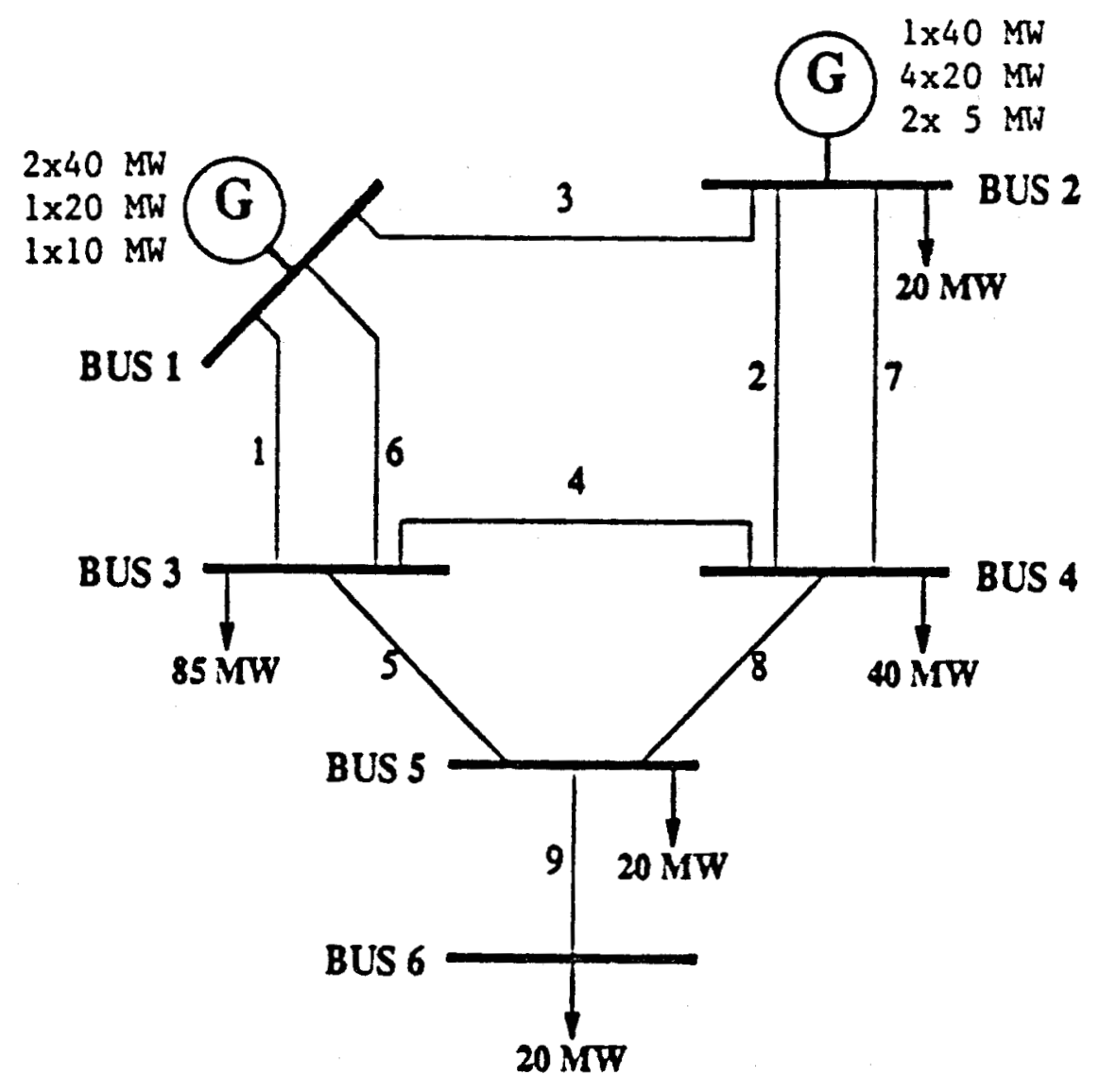

Figure 2.4: Single line diagram of the RBTS 


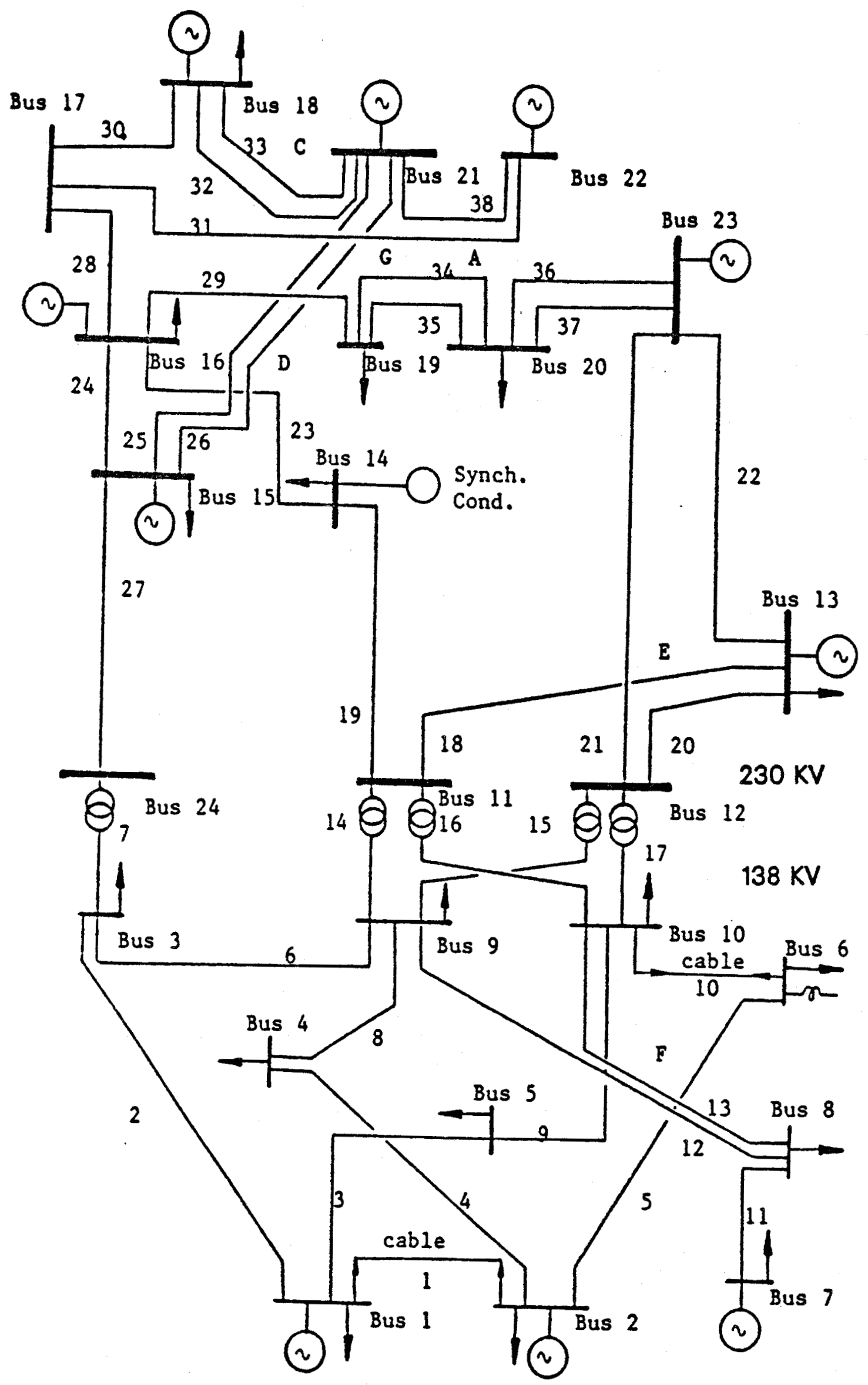

Figure 2.5: Single line diagram of the IEEE-RTS 


\subsection{SYSTEM STUDIES}

The test systems described in this chapter are used to compare the relative merits of network flow, dc load flow and decoupled load flow network solutions for adequacy studies. The load bus indices and the system indices described earlier have been computed using a digital computer program, COMREL, which has been developed at the University of Saskatchewan. Sherman-Morrison correction formulas have been utilized in the studies for the line and combined line and generator outages.

\subsubsection{The RBTS}

The total number of contingencies of various types for the RBTS are shown in Table 2.2. The probabilities and frequencies of load point failure are shown in Table 2.3. Both tables show results from the three methods of analysis. These tables indicates that the dc load flow method (DCLF) provides optimistic adequacy indices compared with those obtained using the decoupled method (ACLF). This is due to the fact that the dc load flow method provides an approximate estimate of the line flows without including any estimate of the bus voltages and MVAr limits of the generating units, etc., etc.. The network flow (NF) method provides the most pessimistic results in the form of probabilities and frequencies for most of the buses. In this case, load is curtailed proportionately from all buses in a capacity deficient area.

Table 2.4 shows additional bus indices and Table 2.5 shows the system indices obtained using the three methods. No simple conclusion can be drawn from these results. These tables indicate that results of the NF method are more optimistic than the other two methods since the NF method is not affected by the line parameters ( $R, X$ and $B)$ and the bus parameters ( $\mathrm{V}$ and $\delta$ ) hence the total load curtailment from different buses is lower than that determined with the other two methods. Between the dc and the ac load flow methods, the dc load flow method results in optimistic adequacy indices at some buses while at other buses the results are pessimistic. This is due to the algorithms used for corrective actions. The line over-load alleviation technique, generation rescheduling for non-convergent and voltage limit violation problems and the amount of load curtailment at different buses depend on bus voltage magnitude, bus voltage angle and the degree of line overloads etc., etc.. These quantities are all different for the two methods. 
Table 2.2: Total number of different contingencies for the RBTS

Number of

NF DCLF ACLF

generator contingencies considered

line contingencies considered

generator-line contingencies considered

voltage violation contingencies

MVAr limits violation contingencies

no convergence contingencies

load curtailment contingencies

isolation contingencies

split network contingencies

firm load curtailment contingencies

CPU time in seconds on MicroVax 3600

66
45
99
0
0
0
45
20
1
21

66

66

$45 \quad 45$

45

$99 \quad 99$

99

$0 \quad 0$

$0 \quad 0$

1

$0 \quad 0$

0

$\begin{array}{lll}45 & 58 & 63\end{array}$

$20 \quad 20 \quad 20$

$\begin{array}{rrr}1 & 1 & 1\end{array}$

$0.97 \quad 1.68$

2.43

Table 2.3: Probability and frequency of failure for up to second level contingencies - RBTS

\begin{tabular}{lcccccc} 
Bus & \multicolumn{3}{c}{ Probability } & & & \multicolumn{2}{c}{ Frequency } \\
& NF & DCLF & ACLF & NF & DCLF & ACLF \\
\hline & & & & & & \\
2 & 0.00743208 & 0.00539921 & 0.00539921 & 2.99220657 & 2.11278009 & 2.11278009 \\
3 & 0.00746534 & 0.00771669 & 0.00778914 & 3.05229759 & 3.38236403 & 3.51306963 \\
4 & 0.00746534 & 0.00539921 & 0.00544047 & 3.05229759 & 2.11278009 & 2.18719864 \\
5 & 0.00746637 & 0.00000103 & 0.00004460 & 3.05416536 & 0.00186768 & 0.08048595 \\
6 & 0.00858125 & 0.00111591 & 0.00111823 & 4.14762497 & 1.09532666 & 1.09952664
\end{tabular}


Table 2.4: Load point indices for up to second level contingencies - RBTS

\begin{tabular}{ccccc}
\hline & $\begin{array}{c}\text { Load Curtailed } \\
\text { (MW) } \\
\text { Bus }\end{array}$ & Energy Curtailed & & Duration of Load \\
(MWh) & Curtailment (Hrs) \\
DCLF ACLF & NF & DCLF ACLF & NF DCLF ACLF \\
\hline
\end{tabular}

Expected Values :

$\begin{array}{rrrrrrrrrr}2 & 3.19 & 4.47 & 4.43 & 68.96 & 100.14 & 99.97 & 65.10 & 47.30 & 47.30 \\ 3 & 13.85 & 33.83 & 35.57 & 294.56 & 669.80 & 679.14 & 65.40 & 67.60 & 68.23 \\ 4 & 6.52 & 8.95 & 8.97 & 138.62 & 200.32 & 200.52 & 65.40 & 47.30 & 47.66 \\ 5 & 3.30 & 0.04 & 0.04 & 69.49 & 0.18 & 0.20 & 65.41 & 0.01 & 0.37 \\ 6 & 25.17 & 21.91 & 21.91 & 264.82 & 195.51 & 195.51 & 75.17 & 9.78 & 9.78\end{array}$

Maximum Values :

$\begin{array}{rrrrrrrrrr}2 & 2.70 & 17.24 & 6.31 & 60.87 & 90.08 & 90.08 & 24.40 & 24.40 & 24.40 \\ 3 & 11.85 & 30.62 & 30.62 & 258.69 & 622.03 & 622.13 & 24.40 & 22.52 & 22.52 \\ 4 & 5.58 & 36.27 & 40.00 & 121.74 & 180.17 & 193.59 & 24.40 & 24.40 & 24.40 \\ 5 & 20.00 & 20.00 & 20.00 & 96.74 & 96.74 & 96.74 & 24.40 & 4.84 & 4.86 \\ 6 & 20.00 & 20.00 & 20.00 & 187.42 & 187.42 & 187.42 & 24.40 & 9.37 & 9.37\end{array}$

Average Values :

\begin{tabular}{rrrrrrrrrr}
2 & 1.07 & 2.12 & 2.10 & 23.05 & 47.40 & 47.32 & 21.64 & 22.41 & 22.59 \\
3 & 4.54 & 10.00 & 10.13 & 96.51 & 198.03 & 193.32 & 21.26 & 19.80 & 19.09 \\
4 & 2.14 & 4.23 & 4.10 & 45.41 & 94.81 & 91.68 & 21.26 & 22.39 & 22.35 \\
5 & 1.08 & 20.00 & 0.53 & 22.75 & 96.74 & 2.56 & 21.08 & 4.84 & 4.84 \\
6 & 6.07 & 20.00 & 18.89 & 63.85 & 178.49 & 178.49 & 10.52 & 8.93 & 8.93 \\
\hline
\end{tabular}

\subsubsection{The IEEE-RTS}

The variation in the values of the basic bus adequacy indices, probability of failure, frequency of failure, expected load curtailed (MW), expected energy not supplied (MWh) using the NF, DCLF and ACLF solution techniques are shown in Tables 2.6 and 2.7. The total number of contingencies considered and the computation time required by the three methods are given in Table 2.8. In the case of generating unit (or line) outages, all outages involving two or less than two generating units (or lines) have been considered. In the case of combined generator and line outage situations, all events involving one generating unit and one line have been considered. The variation in system indices using the three methods are given in Table 2.9 . 
Table 2.5: System indices for up to second level contingencies - RBTS

NF DCLF ACLF

IEEE Indices :

\begin{tabular}{|c|c|c|c|}
\hline BPII & 0.28122 & 0.37398 & $0.38334 \mathrm{MW} / \mathrm{MW}-\mathrm{Yr}$ \\
\hline BPI & 4.52129 & 6.30242 & 6.35316 MWh/MW-Yr \\
\hline $\mathrm{MCI}$ & 11.61084 & 14.31430 & 14.29842 MW/Dist. \\
\hline ty Index & 271.27700 & 378.14500 & 381.19000 System-Min. \\
\hline $\mathrm{MB}$ & 0.00051613 & 0.00071945 & 0.00072525 \\
\hline
\end{tabular}

Average Values (per load point per year) :

No. of load curtailments

3.25972

1.74102

1.79693

No. of voltage violations

before reactive comp. added

after reactive comp. added

No. load curtailed

0.00000

0.00000

0.00252

0.00000

0.00000

0.00000

10.40501

13.83726

14.18367 MW

No. energy curtailed

167.28763

233.18942

67.29494

34.39536

235.06697 MWh

$34.66684 \mathrm{Hrs}$

Maximum values :

Load curtailed

Energy curtailed
40.00

563.03
53.51

653.12
47.18 MW

653.12 MWh

In the case of the NF method, as seen from Table 2.6, both the probability and frequency of failure are almost equal for each bus in the system. This is due to the load curtailment philosophy which interrupts load proportionately at buses in the sink area which covers all buses under an event resulting from the outages of the generating units. The expected values of load curtailed and the energy not supplied are, however, not equal for all buses. These values follow the load profile of the system very closely.

As seen from Table 2.8, the computation time for the ACLF method is several times more than that of the NF method. The computation time for the DCLF is in between as 
expected. It should be also noted that all the indices obtained using the NF, DCLF and ACLF methods are quite different from each other. This is due to the failure criteria defined for the three methods, line overload alleviation algorithm, generation rescheduling, etc.. The NF method is not affected by the line parameters ( $R, X$ and $B$ ) and the bus parameter ( $\mathrm{V}$ and $\delta$ ). The ACLF method is nonlinear in nature while the network flow method is linear. The NF or DCLF methods do not provide any information regarding the quality of the power supply. There are situations when a bus is in difficulty due to voltage violations but the NF and DCLF methods are unable to detect it. The ACLF method, on the other hand, gives a more complete picture of the network performance under outages. Buses $3,4,6,7$ and 8 in the IEEE-RTS experience voltage problems due to several contingencies and for this reason the indices for these buses are higher when using the ACLF method than with the DCLF method. It is also clear from these tables that the load and energy curtailments for the buses in the north region are much higher than those at the buses in the south region with both DCLF and ACLF methods. Due to the load curtailment procedure, the buses in the north region experience load interruption with the removal of two large generating units from the system. Buses in the south experience load curtailment only when three generating units are out or at least one line in combination with other components are out. The load curtailment at buses $3,4,6,8,9$ and 10 in the south region for the second level contingencies is due to the outage of line 11 in combination with the outage of any large generating units in the system. The buses in the north are affected mainly due to generating unit outages and that is why the indices for the buses in the north region are more or less similar with both the DCLF and ACLF methods. The DCLF method gives optimistic results for those buses in the south region which suffer voltage problems under first level outages. In order to obtain more realistic results it is necessary to use the ACLF method. 
Table 2.6: Probability and frequency of failure for up to second level contingencies - IEEE-RTS

\begin{tabular}{rrrlrrrr}
\hline & & Probability & & & & \multicolumn{3}{c}{ Frequency } \\
Bus & NF & DCLF & ACLF & NF & DCLF & ACLF \\
& & & & & & & \\
\hline & & & & & & & \\
1 & 0.0195998 & 0.0000000 & 0.0000000 & 8.3919411 & 0.0000000 & 0.0000000 \\
2 & 0.0195998 & 0.0000000 & 0.0000000 & 8.3919411 & 0.0000000 & 0.0000000 \\
3 & 0.0195998 & 0.0000001 & 0.0013261 & 8.3919411 & 0.0000871 & 0.7294987 \\
4 & 0.0195998 & 0.0000000 & 0.0002594 & 8.3920259 & 0.0000845 & 0.3191945 \\
5 & 0.0195998 & 0.0000000 & 0.0000002 & 8.3920088 & 0.0000675 & 0.0002855 \\
6 & 0.0195999 & 0.0000002 & 0.0007672 & 8.3921719 & 0.0002303 & 0.4646028 \\
7 & 0.0195783 & 0.0000000 & 0.0001987 & 8.3665123 & 0.0000000 & 0.2449221 \\
8 & 0.0195998 & 0.0000349 & 0.0001987 & 8.3919411 & 0.0426340 & 0.2449221 \\
9 & 0.0195998 & 0.0000349 & 0.0001201 & 8.3919411 & 0.0427211 & 0.0966149 \\
10 & 0.0195998 & 0.0000349 & 0.0000832 & 8.3919411 & 0.0426340 & 0.0756943 \\
13 & 0.0195998 & 0.0237410 & 0.0238874 & 8.3919411 & 11.1682463 & 11.2722664 \\
14 & 0.0195998 & 0.0026305 & 0.0026311 & 8.3920412 & 1.3639969 & 1.3648835 \\
15 & 0.0195998 & 0.0185074 & 0.0185075 & 8.3919411 & 8.3746128 & 8.3748169 \\
16 & 0.0195998 & 0.0052610 & 0.0052619 & 8.3919411 & 2.7279072 & 2.7291574 \\
18 & 0.0195998 & 0.0301216 & 0.0301216 & 8.3919411 & 13.8475304 & 13.8475304 \\
19 & 0.0195998 & 0.0026305 & 0.0026316 & 8.3919411 & 1.3638970 & 1.3654863 \\
20 & 0.0195998 & 0.0138662 & 0.0138668 & 8.3919411 & 6.4632931 & 6.4641771 \\
& & & & & & \\
\hline
\end{tabular}

\subsection{CONTINGENCY EVALUATION CUTOFF CRITERIA}

In order to obtain more indicative adequacy indices, outages of individual generating units, lines and transformers in combination with generating units, lines and transformers should be considered in an adequacy evaluation study. Treating generating units, lines and transformers as separate elements increases the flexibility of an adequacy evaluation approach but the number of possible states which represent outage contingencies also increases tremendously. Table 2.10 shows the total number of possible states for the two test systems studied in this thesis.

It is essential to consider all contingency states in calculating the adequacy indices of a power system. It is, however, quite clear from Table 2.10 that it is impossible to 
Table 2.7: Expected load and energy curtailed for up to second level contingencies - IEEE-RTS

\begin{tabular}{|c|c|c|c|c|c|c|}
\hline \multirow[b]{2}{*}{ Bus } & \multicolumn{3}{|c|}{$\begin{array}{l}\text { Load Curtailed } \\
\text { (MW) }\end{array}$} & \multicolumn{3}{|c|}{$\begin{array}{c}\text { Energy Curtailed } \\
\text { (MWh) }\end{array}$} \\
\hline & NF & DCLF & ACLF & NF & DCLF & ACLF \\
\hline 1 & 37.48 & 0.00 & 0.00 & 836.70 & 0.00 & 0.00 \\
\hline 2 & 33.66 & 0.00 & 0.00 & 751.48 & 0.00 & 0.00 \\
\hline 3 & 62.47 & 0.01 & 4.47 & 1394.50 & 0.03 & 57.58 \\
\hline 4 & 25.69 & 0.01 & 1.57 & 573.32 & 0.03 & 10.51 \\
\hline 5 & 24.64 & 0.00 & 0.00 & 550.07 & 0.02 & 0.02 \\
\hline 6 & 47.23 & 0.03 & 2.21 & 1053.82 & 0.20 & 28.37 \\
\hline 7 & 43.36 & 0.00 & 0.00 & 968.23 & 0.00 & 0.00 \\
\hline 8 & 59.34 & 0.60 & 0.60 & 1324.78 & 4.37 & 4.36 \\
\hline 9 & 60.73 & 0.62 & 6.28 & 1355.77 & 4.51 & 68.21 \\
\hline 10 & 67.67 & 0.69 & 3.81 & 1510.71 & 4.98 & 45.38 \\
\hline 13 & 91.96 & 314.13 & 319.69 & 2053.02 & 6147.30 & 6216.27 \\
\hline 14 & 67.34 & 10.55 & 10.58 & 1503.06 & 177.96 & 178.39 \\
\hline 15 & 110.01 & 308.16 & 308.29 & 2455.87 & 6657.16 & 6659.61 \\
\hline 16 & 34.70 & 12.30 & 12.34 & 774.72 & 207.72 & 208.21 \\
\hline 18 & 115.56 & 776.19 & 776.81 & 2579.83 & 16879.55 & 16893.63 \\
\hline 19 & 62.81 & 9.82 & 9.87 & 1402.25 & 165.95 & 166.44 \\
\hline 20 & 44.42 & 124.06 & 124.07 & 991.65 & 2617.53 & 2617.52 \\
\hline
\end{tabular}

attempt to calculate the contribution of all states. In order to limit the number of contingencies, fixed criteria such as the selection of a level of contingency and/or variable criteria such as probability/frequency cutoff limits and/or ranking cutoff limits etc. are presently used. The selection of a criterion depends upon various factors such as the size of the system, the probabilities and frequencies of the outage events, the severity associated with an outage event, the purpose of the adequacy studies and the computation time required to evaluate each outage contingency. The effects of different cutoff criteria on the adequacy indices are discussed in Reference [70]. In addition to selecting an appropriate cutoff criterion, an effective way to reduce the computation time is to sort out the identical elements and calculate the adequacy indices by solving the ac load flow for only one contingency. The contribution of the remaining identical contingencies is calculated by multiplying the adequacy indices for this contingency by the number of identical contingencies. 
Table 2.8: Total number of different contingencies for up to second level - IEEERTS

Number of

NF DCLF ACLF

generator contingencies considered

line contingencies considered

528

741

528

741

528

generator-line contingencies considered

1216

voltage violation contingencies

0

1216

741

MVAr limits violation contingencies

no convergence contingencies

0

0

1216

load curtailment contingencies

isolation contingencies

split network contingencies

firm load curtailment contingencies

\subsubsection{Contingency Level Cutoff Criteria}

Recent investigations $[47,70]$ have indicated that the inclusion of high level contingencies is necessary when calculating adequacy indices for relatively large networks. There is, however, no definite contingency level that can be specified as sufficient for all systems. The maximum depth can be selected according to a desired probability cutoff based on the component outage data.

The sum of the probabilities of all independent outage contingencies up to the fourth contingency level for the two test systems are shown in Table 2.11. The sum of the probabilities for all possible outage contingencies in any system is unity. As seen from Table 2.11, for the RBTS, the sum of the probabilities of contingencies up to the second contingency level is close to unity. $99.35 \%$ of the total contingency probability is contributed by the first and second level contingencies and the remaining portion is 
Table 2.9: System indices up to second level contingencies - IEEE-RTS

\begin{tabular}{|c|c|c|c|c|}
\hline & & NF & DCLF & ACLF \\
\hline \multicolumn{5}{|l|}{ IEEE Indices: } \\
\hline BPII & (MW/MW-Yr) & 0.34704 & 0.54637 & 0.55459 \\
\hline BPECI & (MWh/MW-Yr) & 7.74730 & 11.53239 & 11.63316 \\
\hline BPSAMWCI & (MW/Dist.) & 117.85302 & 100.72269 & 101.53795 \\
\hline & (System-Min.) & 464.83800 & 691.94300 & 697.98900 \\
\hline MBPECI & & 0.00088439 & 0.00131648 & 0.00132799 \\
\hline \multicolumn{5}{|c|}{ Avg. Values (Per Load Point Per Year) : } \\
\hline ANLC & & 8.39408 & 2.67282 & 2.69037 \\
\hline \multicolumn{5}{|l|}{ ANVV } \\
\hline before react. $c$ & omp. added & - & - & 0.11807 \\
\hline after react. co & mp. added & - & - & 0.00000 \\
\hline ALC & (MW) & 58.18074 & 91.59813 & 92.97491 \\
\hline AEC & (MWh) & 1298.81152 & 1933.37134 & 1950.26453 \\
\hline ANHLC & (Hrs) & 171.68298 & 49.91300 & 50.13282 \\
\hline \multicolumn{5}{|c|}{ Maximum Indices : } \\
\hline MLC & (MW) & 245.00 & 293.09 & 352.87 \\
\hline MEC & (MWh) & 5949.12 & 7116.93 & 7125.70 \\
\hline
\end{tabular}

Table 2.10: Total number of possible states for the two test systems

\begin{tabular}{lcrrrrr}
\hline Test & \multicolumn{6}{c}{ Number Of States Up To } \\
System & $\begin{array}{c}\text { Total } \\
\text { Components }\end{array}$ & 1st & $\begin{array}{c}\text { Contingency Level } \\
\text { 2nd }\end{array}$ & 3rd & 4th & Total Possible \\
& & & & & & \\
\hline RBTS & 20 & 20 & 210 & 1350 & 6195 & 1048575 \\
IEEE-RTS & 70 & 70 & 2485 & 57225 & 974120 & $11805918 * 10^{14}$ \\
\hline
\end{tabular}


Table 2.11: Sum of the probabilities for different contingency levels for the two test systems

\begin{tabular}{llll}
\hline Contingency & Probability & $\begin{array}{l}\text { \% Of Total } \\
\text { Contingency } \\
\text { Probability }\end{array}$ & $\begin{array}{l}\text { Contribution } \\
\text { From Line }+ \\
\text { Generator Cont. }\end{array}$ \\
\hline
\end{tabular}

\section{For The RBTS}

$\begin{array}{llll}\text { all comp. in } & 0.79378355 & & \\ \text { total cont. } & 0.20621645 & & \\ \text { 1st level cont. } & 0.97890913 & 89.77 & \\ \text { 2nd level cont. } & 0.99866265 & 99.35 & 0.41713240^{*} 10^{-2} \\ \text { 3rd level cont. } & 0.99994200 & 99.97 & 0.41423630^{*} 10^{-3} \\ \text { 4th level cont. } & 0.99997926 & 99.99 & 0.56862591 * 10^{-5}\end{array}$

For The IEEE-RTS

$\begin{array}{llll}\text { all comp. in } & 0.23045120 & & \\ \text { total cont. } & 0.76954880 & & \\ \text { 1st level cont. } & 0.58153069 & 45.62 & \\ \text { 2nd level cont. } & 0.83524644 & 78.59 & 0.88586016^{*} 10^{-2} \\ \text { 3rd level cont. } & 0.95110387 & 93.65 & 0.63405256^{*} 10^{-2} \\ \text { 4th level cont. } & 0.98643482 & 98.24 & 0.62273158 * 10^{-3}\end{array}$

cont. $=$ contingency or contingencies comp. $=$ components

contributed by the contingencies beyond the second contingency level. In the case of IEEE-RTS system, the sum of the probabilities of contingencies up to the second contingency level is $78.59 \%$ and up to fourth contingency level is $98.24 \%$. The remaining $1.76 \%$ is contributed by the contingencies beyond the fourth contingency level. It can therefore be reasonably deduced that as the size of a system increases, the calculation of adequacy indices by considering only low level contingencies will provide optimistic results. This is due to the fact that as the number of components in a system increases, the probability and the frequency of an independent outage involving three or more components increases to the point at which they can not be ignored. The testing of 
higher level independent outages is, therefore, necessary when calculating adequacy indices. In this study, independent outages up to the third contingency level plus fourth level generating unit outages are considered. The reasons for not considering fourth level outages of transmission lines and fourth level combinations of transmission lines and generating units (i.e. 3 lines, 1 generator +2 lines, 2 generators +1 line, 3 generators) are as follows:

1. The probability contribution of these outages are $5.6862591^{*} 10^{-06}$ and $6.2273158 * 10^{-04}$ which are $0.002757422 \%$ and $0.080921649 \%$ respectively of the total contingency probability for the RBTS and the IEEE-RTS respectively.

2. The total number of fourth level states, 4,515 for the RBTS and 880,935 for the IEEE-RTS, is very large and for this reason alone the computation time even with the approximate selection process discussed in Chapter 3 will be very high.

\subsection{APPROXIMATE SOLUTION CONSIDERATIONS}

It has been shown [92] that the inclusion of high level independent outages cannot be ignored in the calculation of more representative adequacy indices. This requirement, however, involves large CPU time and the time increases tremendously as the outage level increases. The CPU times required for the solution of different contingency levels for the two test systems are shown in Table 2.12. In the case of fourth level contingencies, transmission outages were restricted to a third level and generating units considered for a fourth level. The execution time involved in adequacy analysis varies with the following factors:

1. the size of the system,

2. the number of simultaneous independent outages considered and

3. the load flow technique adopted for analyzing each outage case.

Practical systems are usually quite large and contain many components. An adequacy index computation program must fit within the memory capabilities of the available computer and be executable in a reasonable time. Memory requirements, which earlier played an important role in the practical application of network solution techniques, are no longer much of an issue. The execution time required to evaluate the indices for a large system limits the contingency level which can be considered. 
As noted earlier, a load flow analysis is performed for each selected contingency in order to obtain the adequacy indices. The bulk of the computation time is, therefore, utilized in the load flow analysis of each outage condition. The purpose of each load flow solution is to identify potential voltage violations, line overloads, etc. and therefore high solution accuracy associated with detailed ac load flow analysis is not necessary. Computing speed and acceptable accuracy of the solution have a high priority due to the large number of cases that may have to be examined.

Table 2.12: CPU time required for solution of the two test systems at various contingency levels (on MicroVax 3600 digital computer)

\begin{tabular}{lcrrr}
\hline $\begin{array}{l}\text { Contingency } \\
\text { Level }\end{array}$ & Min. & Sec. & \multicolumn{2}{c}{ IEEE-RTS } \\
& & & & \\
& & & & \\
\hline & 0 & 0.69 & 0 & 4.80 \\
1st level & 0 & 2.43 & 2 & 10.05 \\
2nd level & 0 & 12.39 & 54 & 48.72 \\
3rd level & 0 & 14.19 & 59 & 24.31 \\
4th level & & & & \\
\hline
\end{tabular}

It is also not necessary to perform an ac load flow for all contingencies considered, since not all of them create system problems. For example, in the case of the RBTS, no single component outage creates a problem in the system except that of line 9. Bus 6 experiences isolation for this outage. The number of contingencies which create system problems at various contingency levels for the two test systems are shown in Table 2.13. As seen from Table 2.13, a significant amount of time can be saved if a list of these contingencies which are creating problems can be found using an approximate but fast method. The contingencies from this list can then be tested using more accurate methods. Different methods which can be used for this purpose are discussed in the following chapter. 
Table 2.13: Number of contingencies creating system problems for the two test systems

\begin{tabular}{lcccc}
\hline $\begin{array}{l}\text { Contingency } \\
\text { Level }\end{array}$ & $\begin{array}{c}\text { Total } \\
\text { Contingencies } \\
\text { Studied }\end{array}$ & $\begin{array}{c}\text { Contingencies } \\
\text { Creating } \\
\text { Problems }\end{array}$ & $\begin{array}{c}\text { contal } \\
\text { Contingencies } \\
\text { Studied }\end{array}$ & $\begin{array}{c}\text { Contingencies } \\
\text { Creating } \\
\text { Problems }\end{array}$ \\
\hline 1st level & 20 & 1 & 70 & 5 \\
2nd level & 210 & 39 & 2485 & 283 \\
3rd level & 1350 & 345 & 57225 & 7213 \\
4th level & 1680 & 506 & 93185 & 8845 \\
& & & & \\
\hline
\end{tabular}

\subsection{SUMMARY}

The contingency enumeration approach is a basic technique for composite system adequacy assessment. This approach involves the selection and evaluation of contingencies, the classification of these contingencies in accordance with selected failure criteria and the accumulation of adequacy indices. The computation time required to obtain a solution in a particular system study is dependent on a number of factors of which the system solution technique and the contingency level are key elements. The solution technique is dictated by the intent behind the studies and it is not possible to obtain comprehensive system information from approximate or linearized solution techniques. This chapter illustrates the effect on the calculated indices and on the computer solution time of using network flow, dc load flow and ac load flow techniques in composite system adequacy evaluation.

The inclusion of high level contingencies cannot be ignored in the calculation of representative adequacy indices as the size of the system increases. There is no fixed contingency level that can be specified as being sufficient for all systems. The required maximum contingency level can be obtained from a knowledge of the component outage data and the desired probability cutoff. It has been shown that the DCLF method gives 
optimistic results for the buses in the south region of the IEEE-RTS which suffer voltage problems even with first level outages. In order to obtain realistic results, it is necessary to use the ACLF method. The CPU time increases considerably when the ACLF method is utilized for contingency analysis including high level outages. The inclusion of high level outages may however be very important especially for large power networks. It has been shown that the number of contingencies and hence the CPU time increases tremendously as the contingency depth increases, especially for large networks. This chapter also illustrates that only a small percentage of the total possible contingencies create system problems. A significant amount of CPU time can be saved if a list of those contingencies which are creating problems can be found using an approximate fast method. The contingencies from this list can be tested using more accurate methods. The different methods used for this purpose are discussed in the next chapter. 


\section{FAST APPROXIMATE ADEQUACY EVALUATION}

\subsection{INTRODUCTION}

A detailed adequacy evaluation of a composite generation and transmission system involves the simulation and computation of the system conditions for each possible outage condition in the system in order to determine the voltage violations, line and generator overloads, violation or generator MVAr limits etc.. The inclusion of high level contingencies is necessary in calculating the adequacy of a large power system network. These contingencies involve both independent outages and dependent events resulting from common mode outages and station originated failures [93]. As the size of a system increases, it becomes difficult to determine the adequacy of the system under all credible outage conditions [93] due to the tremendous increase in computation time required for the solution of the network. Under these conditions, it may not be practical to attempt to solve very large networks using ac load flow techniques. On the other hand, conducting adequacy evaluation by testing a subset of contingency cases selected on the basis of the planner's experience and intuition may also be undesirable due to the possibility of neglecting some critical cases.

One of the most widely used approaches to reduce the computation time when conducting a series of contingency evaluations is to rank the outage contingencies using fast techniques and then investigate these ranked contingencies using an ac load flow method. This procedure is terminated by an appropriate stopping criterion. It is not usual, to perform a specific analysis of the efficiency and ability of a ranking procedure to performs its intended function. In the application examined in this chapter, the objective is to obtain adequacy indices at each load point in the system. This chapter presents a series of adequacy indices evaluated using different ranking methods. The computation time and the differences in the adequacy indices compared to a more 
complete reference ac solution are presented. Three different performance indices were used to obtain the ranking lists and a consecutive success cutoff criterion was used to provide the stopping rule.

This chapter also presents an alternative approach to contingency determination which is designated as contingency selection. Efficient selection algorithms for outages of generators, transmission lines and combinations of these elements are presented. The application of the method and its comparison with the reference case and the ranking methods illustrated using the two test systems. The Sherman-Morrison correction formula [80] is used to adjust the base case solution to efficiently represent the line and line plus generator outages instead of rebuilding and refactorizing the system admittance matrices for each contingency.

\subsection{ADEQUACY EVALUATION USING RANKING}

A complete procedure for adequacy evaluation of a composite power system requires the evaluation of all 'credible' contingency cases. The computational burden that this procedure places on even the most advanced computer installation has prompted the need for studying procedures for the automatic selection of meaningful contingency cases. A basic and primary objective is to reduce the number of possible cases for detailed consideration and at the same time provide a ranking or ordering of these cases according to severity.

The ultimate goal of a ranking algorithm is to determine which subset of contingencies taken from the set of all possible contingencies will cause system failure. Unfortunately, none of the available ranking methods can attain this goal. At best, they can provide a set of contingencies containing most of the cases causing system failure. Some severe contingencies may be omitted and some that are not severe may be included. This section describes contingency ranking for the calculation of adequacy indices. 


\subsubsection{Performance Indices For Ranking}

A reliable ranking of contingencies according to severe line overloads, voltage problems and MVAr limits etc. can be obtained by solving each contingency by means of an accurate ac load flow. It is not practical to rank the contingencies using a detailed ac load flow because of the computational burden associated with solving and ranking of all possible contingencies. The conventional use of the word "ranking" implies the investigation of all possible contingencies by an approximate algorithm that can reliably indicate which of the contingencies ought to be investigated further using a more accurate ac load flow.

The usual approach to contingency ranking is to define a scalar function called a Performance Index $(P I)$, which provides a measure of system stress. A method is then developed for predicting the change in the $P I$ when a component is on outage. The change in the PI resulting from outages can then be used to rank the outages in the order of their severity. Each of the above two steps comprising the contingency ranking process can become a source of inaccuracy or inefficiency.

\subsubsection{Ranking Of Outage Contingencies}

There are several ranking techniques $[43-52,94,95]$ that have been proposed and implemented for adequacy and security studies. Most of the available methods rank contingencies by calculating a $P I$ which is a composite measure of a system problem in the entire network.

\subsubsection{Ranking Using Line-Overloads}

The performance index method can be applied for ranking using line-overloads. A $P I$ [43] which measures system stress in terms of line overloads is given in Equation 3.1.

$$
P I_{M W}=\sum_{p=1}^{N L} W_{p}\left(\frac{P_{p}}{\bar{P}_{p}}\right)^{2 m}
$$

where:

$W_{p}=$ weighting factors for line $p$, 


$$
\begin{aligned}
P_{p} & =\text { real power flow for line } p, \\
\bar{P}_{p} & =\text { power rating for line } p, \\
m & =\text { an integer and } \\
N L & =\text { number of lines. }
\end{aligned}
$$

The performance index $P I_{M W}$ contains all line flows normalized by their limits. These normalized flows are raised to an even power (by setting $m=1,2, \ldots$ ); thus, the use of the absolute magnitude of the flows is avoided. This index $P I_{M W}$ will have a relatively small value when all lines flows are within their limits, and a high value when there are line overloads. It therefore provides a good measure of the severity of line overloads for a given state of a power system.

Efficient and accurate prediction of the change in the $P I$ when lines are removed has been the subject of many investigations. Early work in this direction was performed by Ejebe-Wollenburg [43] and other researchers $[51,96]$. These authors proposed a methodology for ranking transmission line contingencies by evaluating the normalized sensitivities of a system wide performance index with respect to line outages.

The assumption in the above method is that the higher order terms are negligible in the Taylor series expansion of $P I_{M W}$ as a function of line susceptance. Unfortunately, this is not always the case [50]. The $P I_{M W}$ suggested in Reference [43] as given in Equation 3.1 is not a monotonic function of the susceptance of the lines. This monotonicity condition seems to be at least a necessary condition for a trustworthy performance of the method. The failure of the assumption manifests itself as inaccuracies in the ranked list of contingencies as compared to the ranked list obtained by solving a dc load flow and calculating the value of $P I_{M W}$ directly for each contingency.

The inaccuracies due to ignoring higher order terms was eliminated in a newer technique developed independently by Mikolinnas and Wollenberg [44] and Irisarri and Sasson [45]. This method properly recognizes all the terms in the Taylor series and eliminates most of the misrankings encountered earlier. Even in this case, the automatic contingency selection algorithm was not found to be sufficiently reliable, and as a 
consequence, it was proposed to use a dc load flow which is highly reliable and less computationally demanding than the second-order extension of $P I_{M W}$.

In most cases, $P I_{M W}$ provides a good measure of system stress by increasing in value when severe contingencies occur. However, in some cases, when a single line becomes overloaded while many other branch loadings decrease, it can decrease in value and fail to recognize the overload. This phenomenon, which is called masking, is inherent in $P I_{M W}$. Masking can be reduced by partitioning the lines into subsets and performing rankings on each of the subsets. The partitioning may be done either by voltage levels, by areas or by partitioning into lines and transformers. Masking can also be reduced if the exponent in the real power performance index is increased to a higher value. The second order extension of $P I_{M W}$ is limited to $m=1$. In this thesis, the dc load flow equation is used to rank the line contingencies together with the utilization of the Sherman-Morrison correction for single or higher level line contingencies. A value for $m=5$ has been selected in order to reduce the masking effect.

\subsubsection{Ranking Using Voltage Deviation}

Contingencies can also result in unacceptable changes in bus voltages. It is possible to detect large deviations in system voltages by doing one or perhaps a few iterations using an approximate ac load flow algorithm, such as the decoupled load flow. If a contingency is judged to be severe, the solution process can be continued until a solution of desired accuracy is obtained. On the other hand, if the contingency is judged not to be severe, then the solution process can be terminated before a solution is reached, with a resultant saving in time. This process must be repeated, of course, for each contingency case.

The performance index method can also be used to detect voltage problems. One possible performance index [43] for voltage problems is given in Equation 3.2.

$$
P I_{V}=\sum_{q=1}^{N B} W_{q}\left(\frac{\left|V_{q}\right|-\left|V_{\text {qref }}\right|}{\left|\Delta V_{q}\right|}\right)^{2 m}
$$

where:

$W_{q} \quad$ = weighting factor for bus $q$, 


$$
\begin{aligned}
V_{q} & =\text { voltage at bus } q \\
V_{q r e f} & =\text { reference voltage at bus } q, \\
\Delta V_{q}= & \text { voltage deviation limit at bus q, above which } \\
& \text { voltage deviations are unacceptable and } \\
N B \quad & \text { number of buses. }
\end{aligned}
$$

The voltage deviation $\Delta V_{q}$ represents the threshold above which voltage level deviations are outside their limit. Any contingency load flow with voltage levels outside this limit yields a high value of the index $P I_{V}$. On the other hand, when all the voltage level deviations from the rated (nominal) voltage are within $\Delta V_{q}$, the voltage performance index $P I_{V}$ is small. Thus $P I_{V}$ measures system stress in terms of how far the system voltage profile is deviating from the profile defined by the reference voltages. The above index is limited to the case where voltage limits are symmetrical about $V_{\text {qref }}$ In order to avoid this difficulty the following $P I_{V}[94]$ is used in this thesis

$$
P I_{V}=\sum_{q=1}^{N B} W_{q}\left(\frac{\left|V_{q}\right|-\left|V_{q b}\right|}{\left|\Delta V_{q b}\right|}\right)^{2 m}
$$

where:

$$
\begin{aligned}
V_{q b} & =\frac{V_{q \max }+V_{q \min }}{2} \text { and } \\
\Delta V_{q b} & =\frac{V_{q \max }-V_{q \min }}{2} .
\end{aligned}
$$

A series of other $P I$ were examined in an EPRI report [47] to find an $P I$ expression that was reasonably sensitive to the voltage problem in order to obtain a good ranking for voltage problems. All the $P I$ expressions tested were variations of the expression

where:

$$
P I=\sum_{\text {Lines }} X_{l} P_{l}^{2}
$$

$$
\begin{aligned}
& X_{l}=\text { reactance of line } l \text { and } \\
& P_{l}=\text { real power flow in line } l .
\end{aligned}
$$


which approximate the system $X I^{2}$ losses. The EPRI studies indicated that none of these indices give satisfactory results. In this thesis, the $P I$ given by Equation 3.3 is used to rank the contingencies using voltage deviations. The decoupled equation

$$
[\mathrm{Q}] \approx[\mathrm{Q} / \mathrm{V}]=[\mathrm{B} "][\mathrm{V}]
$$

is used to calculate the change in bus voltages due to line outages. The ShermanMorrison correction formula is used to calculate the change in voltage $[\Delta \mathrm{V}]$.

\subsubsection{Preparation Of A Contingency Ranked List}

The contingency list can be built by running all possible contingencies and then ranking them from their respective $P I$. In this case, it was found [70] that almost all higher level contingencies are ranked high followed by almost all next lower level contingencies and so on. If the process is stopped after $\mathrm{N}$ consecutively no-problem contingencies are found, then it may happen that almost none of the first or second level contingencies will be included in the study. The contribution from a first and second level contingency, if one or more create system problems, will be much higher than the higher level contingencies. This effect can be eliminated by ranking all first level contingencies first and then for each of the first order ranked contingencies, all possible second order contingencies are ranked and so on. In this case, it is assumed that highly ranked primary contingencies lead to many severe contingencies when additional components fail, whereas lower ranked contingencies tend to cause fewer problems when additional failures occur. It has been shown [70] that when first level contingencies are ranked, not only are they ranked in the order of severity but the subsequent second level contingencies will also be more severe below the higher ranked primary contingencies. As a result, when a given number of first level contingencies and the subsequent second level contingencies have been solved, it is likely that the worst second level contingencies as well as first level contingencies have also been analyzed. The same procedure can be applied for third level contingencies.

It is noted in Chapter 2 that the Sherman-Morrison correction formula can be used recursively for higher level contingencies. If the same $\mathbf{N}$ number of contingencies are studied from two contingency lists as noted above, then the CPU time in the first case 
will be higher than that in the second case. This is due to the fact that in the first case the arrangement is random with respect to lines and therefore the correction for each line outage has to be calculated for each individual case. In the second case the correction associated with the outage of the first line remains the same for the associated second level contingencies, and similarly the outage effects of the first two lines are the same for the corresponding third level contingencies. The correction for the first line on outage is only calculated once for the recursive use of the associated second and third level contingencies. Similarly the correction for the second outage is calculated only once for the recursive use of the associated third level contingencies.

The memory requirement for ranking purposes in the first case will be higher than the memory requirement in the second case. In the first case, the minimum memory requirement will be four times $\left({ }_{N L} \mathrm{C}_{1}{ }^{+}{ }_{N L} \mathrm{C}_{2}{ }^{+}{ }_{N L} \mathrm{C}_{3}\right)$ in order to consider contingencies up to the third levels, three times for storing the ranking list and the remainder to store the $P I$. The memory requirement is thus very large. For example, the memory requirement for the IEEE-RTS is $4 \times 9177$ which is equivalent to a matrix of dimension (170x170) for the third level line contingencies only. In the second case, the minimum memory requirement will be equivalent to approximately $4 \times N L$. $N L$ amount of memory will be required to store the first order ranked contingencies and the remaining memory locations are used to store the corresponding second level contingencies, third level contingencies and the PI values. All the memory locations except the memory storage for the first order contingency list can be used for the next first order contingencies.

\subsubsection{Ranking Cutoff Criteria}

Ranking cutoff criteria mainly depend on the preparation of a contingency list. If the contingencies are placed in a contingency rank list from the most severe to the less severe, then the simplest stopping criterion for a contingency enumeration approach is to terminate the contingency evaluation as soon as the load flow for one case shows noproblem. Since none of the ranking lists are absolutely accurate, it is prudent to terminate the contingency evaluation only after a sequence of several no-problem contingencies has been found. This can be controlled by a cutoff parameter $\mathbf{N}$. 
In this thesis, the ranked lists for contingencies are prepared using the following steps:

1. The first level contingencies are ranked or ordered according to the value of the $P I$.

2. The second level contingencies are ranked again according to their severity for each of the first level contingencies. This procedure is followed for the third level contingencies.

After ranking all contingencies up to the third level, the fast decoupled load flow method is utilized to investigate these contingencies. The investigation of contingencies are stopped by using a consecutive success criterion [47] as follows:

"Analysis of third level contingencies, subsequent to a given second level contingency, is truncated i.e. no further outage events from the list are examined by the decoupled load flow method when a given number of successive contingencies, $\mathbf{N}$, has not created any system problem. Similarly, second level contingencies and their associated third level contingencies are analysed until a succession of $\mathbf{N}$ secondary contingencies are encountered in a row in which the first $\mathbf{N}$ third level contingencies caused no system problem. The same procedure is followed for the first level contingencies. For example, if $\mathbf{N}=\mathbf{5}$, the contingencies will be solved until, for five first level contingencies in a row, none of the worst five second level as well as none of the five associated worst third level contingencies caused any system problem."

\subsection{ADEQUACY EVALUATION USING THE SELECTION METHOD}

Adequacy evaluation of a composite generation and transmission system in a practical power network is a complex problem and is computationally quite expensive when all primary contingencies, each with additional levels of higher order 
contingencies, are involved. It may not be practical to solve very large networks with ac load flow techniques. The main limitations to the solution of large networks are the enormously high computation time and storage requirements. The load point and total system indices are calculated in the previous section using ranking methods with different $P I$ and it has been found that ranking does not offer a good trade-off between speed and accuracy for the final solution. In this section, an efficient computational method is presented. The method can be used to examine both continuity and quality of power supply for any type of outage. The proposed method is based on the implementation of the two decoupled equations and provides more accurate adequacy indices with less CPU time than ranking.

\subsubsection{Selection Method For Transmission Line Outages}

An inherent characteristic of any practical electric power transmission system operating in the steady-state condition is the strong interdependence between active powers and bus voltage angles, and between reactive powers and voltage magnitudes. Correspondingly, the coupling between these " $\mathrm{P}-\delta$ " and " $\mathrm{Q}-\mathrm{V}$ " components of the problem is relatively weak. The voltage vectors method uses a series approximation for the sine terms which appear in the system defining equations, to calculate the Jacobian elements and arrive at the two decoupled equations.

$$
\begin{gathered}
{[\mathrm{P}] \approx[\mathrm{P} / \mathrm{V}]=\left[\mathrm{B}^{\prime}\right][\delta]} \\
\text { and }[\mathrm{Q}] \approx[\mathrm{Q} / \mathrm{V}]=\left[\mathrm{B}^{\prime \prime}\right][\mathrm{V}]
\end{gathered}
$$

where:

[P] = Vector of bus real power injections,

[Q] = Vector of bus reactive power injections,

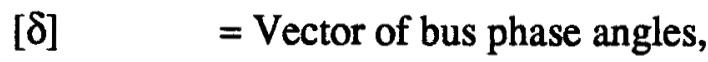

[V] = Vector of bus voltage magnitudes,

$\mathrm{B}_{p q}{ }^{\prime} \quad=-\mathrm{b}_{p q}$ of transmission lines,

$\mathrm{B}_{p p}{ }^{\prime}=\sum \mathrm{b}_{p q}$, 


$$
\begin{array}{ll}
\mathrm{B}_{p q}{ }^{\prime \prime} & =-\mathrm{b}_{p q} \text { and } \\
\mathrm{B}_{p p}{ }^{\prime \prime} & =\sum \mathrm{b}_{p q}-\mathrm{b}_{c p}-\sum \mathrm{b}_{s h p q}
\end{array}
$$

Both matrices $\left[B^{\prime}\right]$ and $\left[B^{\prime \prime}\right]$ are real, sparse and contain only network admittances. Since [B'] and [B"] are constant, they need be triangulated only once, thus reducing the computation time. The vector $[\Delta \delta]$, change in angle $[\delta]$, can be easily obtained under any line contingency using the Sherman-Morrison correction formula or by applying the method described in Chapter 4 to the base solution. The correction formulas avoid the need for rebuilding and retriangularizing the susceptance matrices for an outage event. Using the vector $[\Delta \delta]$, the new bus angles can be computed using Equation 3.6.

$$
[\delta]_{\text {new }}=[\delta]_{\text {base }}+[\Delta \delta]
$$

Power flow in a transmission line is determined using the updated values of bus angles $[\delta]_{n e w}$. The line flow is then checked against its specified power capacity. If the line flow is greater than the capacity, the outage contingency is designated as a problem contingency and is routed to the main routine for further investigation using the fast decoupled ac load flow. No further line flows for that problem creating contingency are either calculated or compared. If no line is found to be overloaded, then the same procedure is repeated using Equation 3.5 to determine the voltage deviation $[\Delta \mathrm{V}]$. The updated values of bus voltages are calculated using Equation 3.7.

$$
[\mathrm{V}]_{\text {new }}=[\mathrm{V}]_{\text {base }}+[\Delta \mathrm{V}]
$$

If for any bus, $\mathrm{V}_{\text {new }}$ is not within the specified limits, the outage event is again selected for further study and examined using the fast decoupled ac load flow method. The flow chart for line outage studies by the selection method is shown in Figure 3.1. A brief summary of the main steps is given below:

1. Solve the power network keeping all the lines in operation and determine the base values of the bus angles and voltages.

2. Using the Sherman-Morrison correction formula or the methods described in Section 4.3.2 to simulate an outage of a transmission line, calculate the deviation in bus angle $[\Delta \delta]$ for the system buses.

3. Update the value of the phase angle at a bus using Equation 3.6 and determine the power flow in a line. If the line flow is greater than the line capacity, go to step 4 , otherwise go to step 5 . 
4. Solve the contingency using the fast decoupled ac load flow method and calculate the adequacy indices. Consider the next contingency and go to step 2.

5. Steps similar to 2 and 3 are repeated to check for the voltage problem. If no voltage problem is experienced, consider the next contingency and go to step 2, otherwise go to step 4 for further study.

\subsection{REFERENCE CASE RESULTS}

It has been noted earlier that contingency evaluation using an ac load flow method provides the most realistic results. In this chapter, therefore, the adequacy indices obtained using a complete ac load flow method have been considered as the reference values for comparison with the ranking and approximate selection methods. The computation time required to obtain the adequacy indices with this ac load flow analysis has been considered as the reference CPU time.

\subsection{COMPARISON OF RANKING AND SELECTION METHODS FOR LINE OUTAGES}

The two test systems described in Chapter 2 were tested using the ranking and selection methods for line outage contingencies. The following is a comparison of the two methods in terms of accuracy and CPU time.

\subsubsection{Using Ranking}

Figure 3.2 and Figure 3.3 show the percentage CPU time, the number of contingencies solved as a percent of the total possible number, and also the number of problem-creating contingencies identified and solved as a percentage of the total number of contingencies that create system problems for the two test systems. These quantities are plotted as a function of $\mathrm{N}$. For example, when $\mathrm{N}=3,2569$ contingencies causing system problems are found out of a total of 3184 such contingencies for the IEEE-RTS. This represents $80.685 \%$ of the cases causing system problems. In order to capture this percentage of problems creating contingencies, it was necessary to solve 4318 out of a possible 9177 contingencies. This represents $47.05 \%$ of the total number of contingencies. These quantities are shown in Figure 3.3. As $\mathrm{N}$ is increased, more severe 


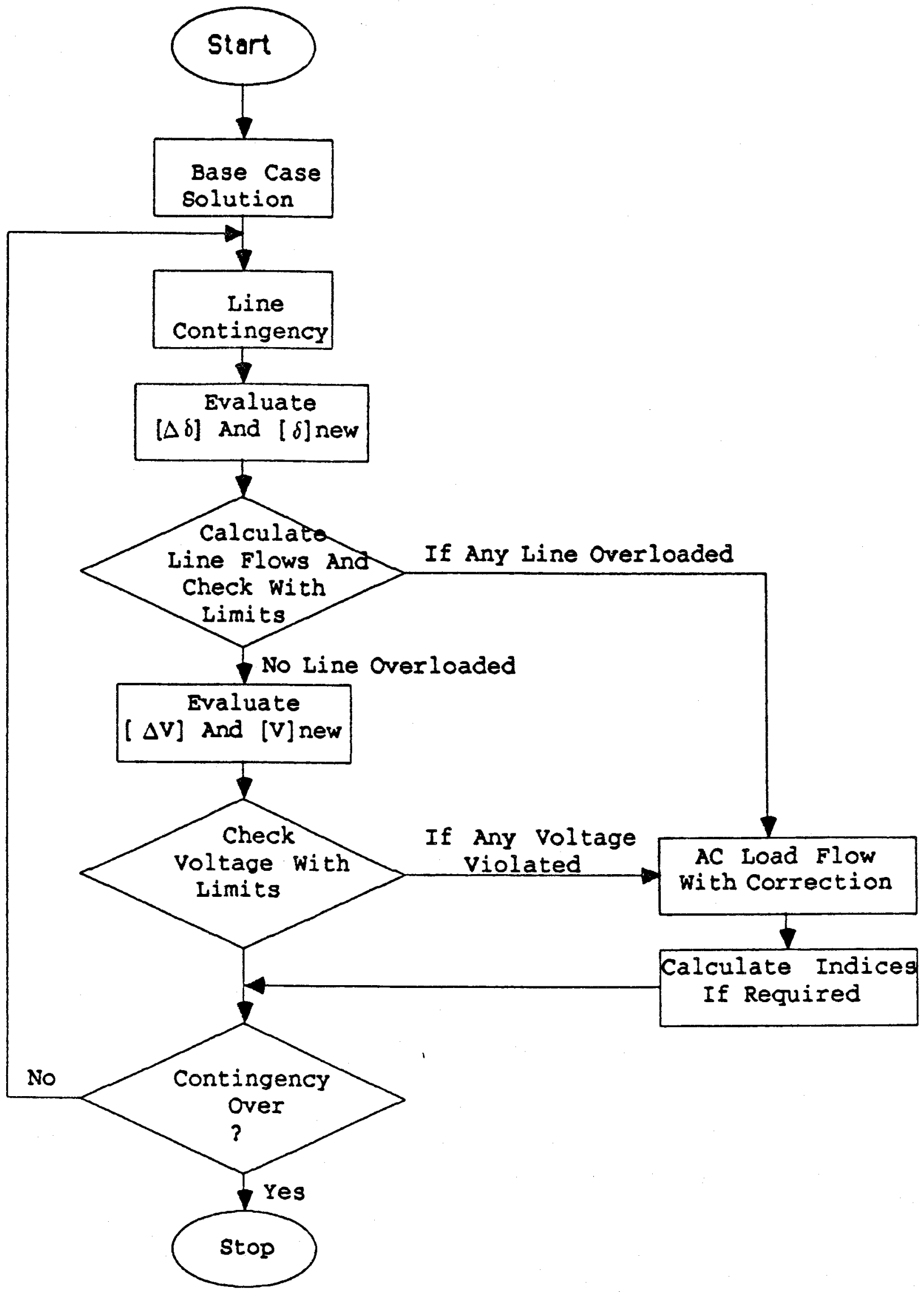

Figure 3.1: Flow chart for the selection of line contingencies 


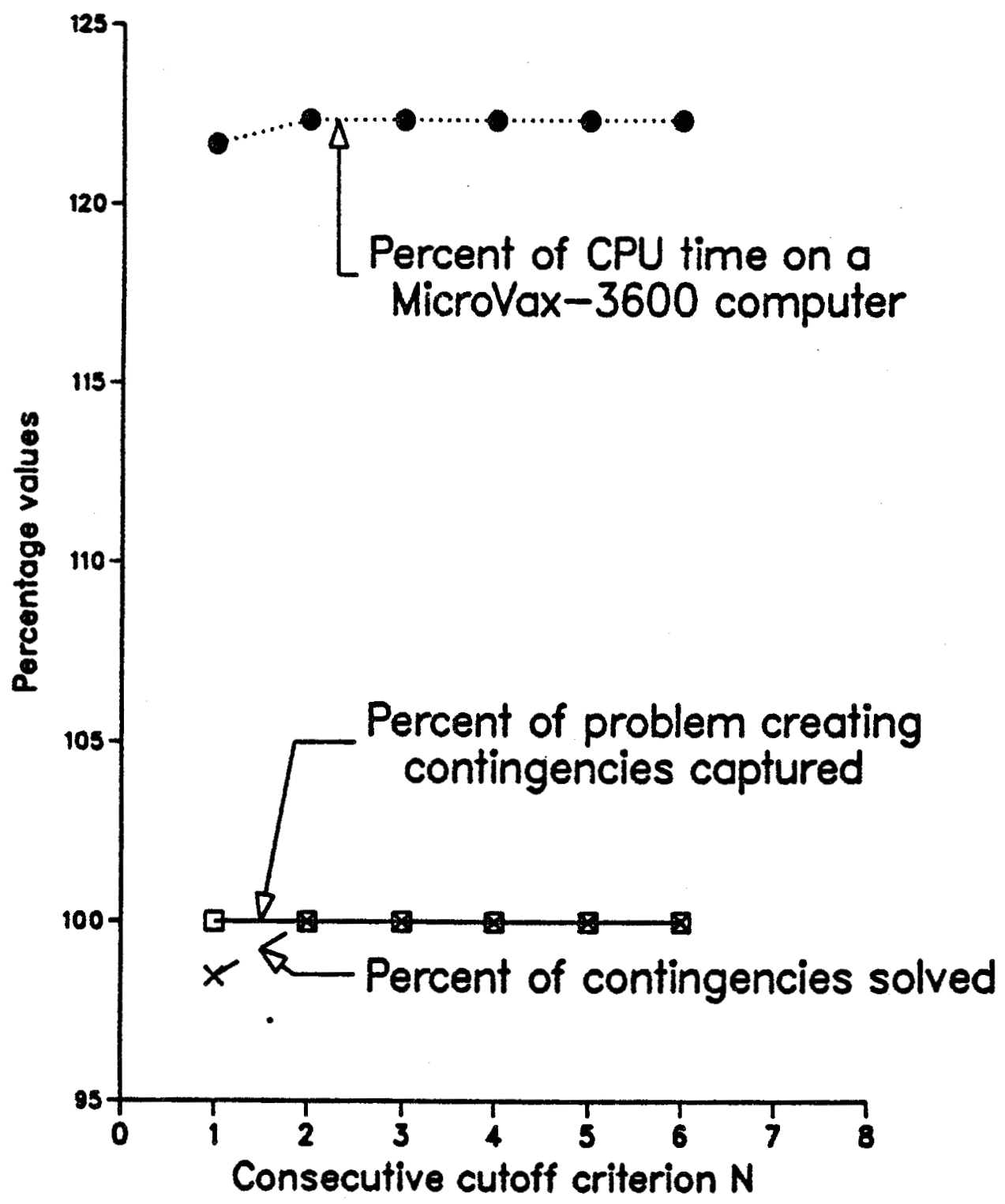

Figure 3.2: Percent of contingencies captured, tested and the CPU time as a function of $\mathrm{N}$ for the RBTS as a percentage of the reference case

contingencies are found, but only at the expense of solving many more contingencies, including some that are not severe and do not cause system problems. From these figures it would appear that for RBTS, $\mathrm{N}=1$ is sufficient to capture all problem creating contingencies whereas $\mathrm{N}>6$ is needed for the IEEE-RTS. In this thesis $\mathrm{N}=3$ is chosen for the IEEE-RTS in order to reduce CPU time. 


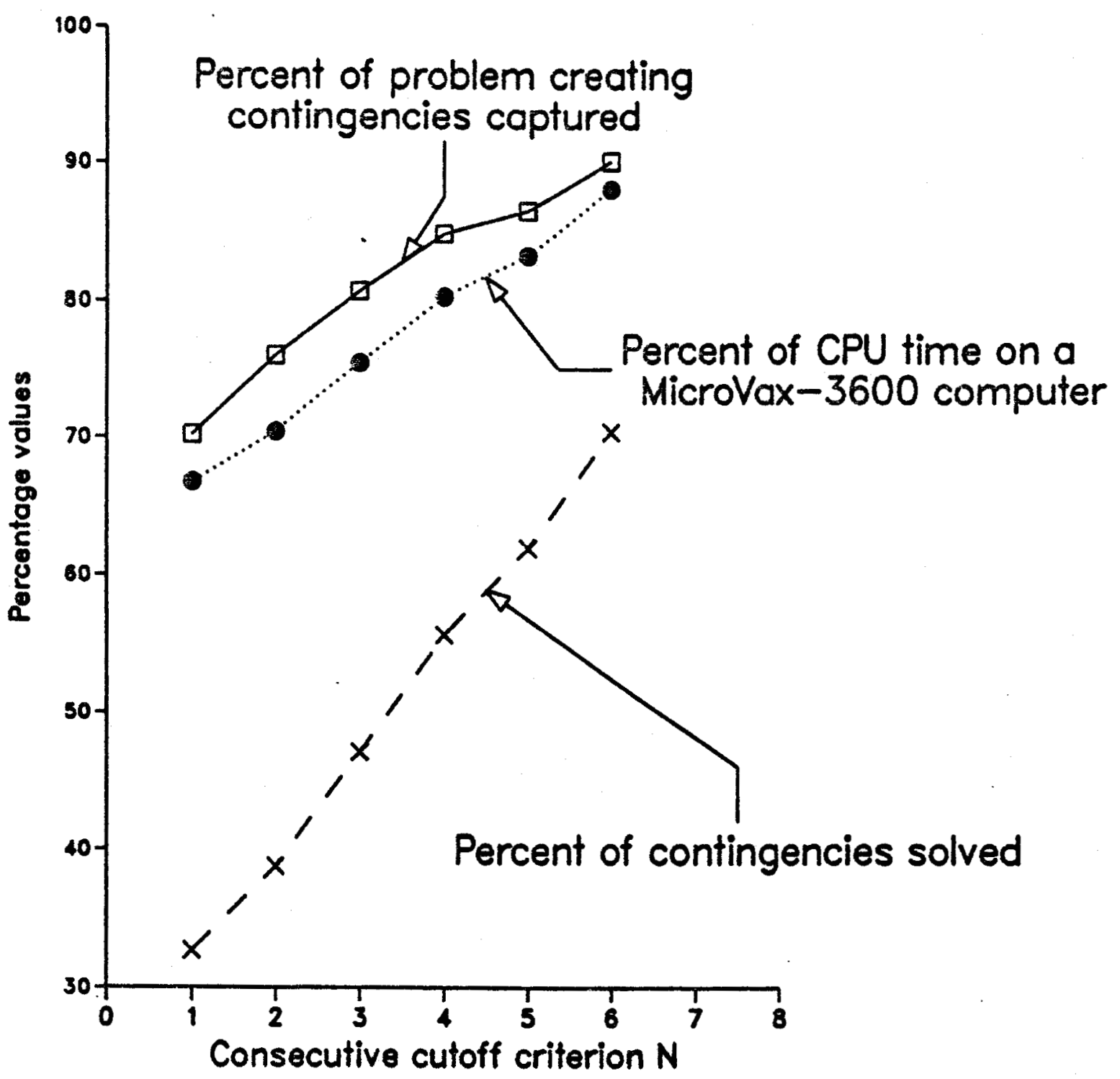

Figure 3.3: Percent of contingencies captured, tested and the CPU time as a function of $\mathrm{N}$ for the IEEE-RTS as a percentage of the reference case

In these studies, it has been assumed that contingencies are ranked from the set which consists of independent line outages up to the third level. Contingencies beyond the third level are not considered for ranking purposes. The outage contingencies are ranked using performance indices $P I_{M W}, P I_{V}$ and $P I_{M W V}$ which is the sum of $P I_{M W}$ and $P I_{V}$. For each case, the contingency list is prepared as noted before and the consecutive success ranking cutoff criterion used as a stopping rule. The adequacy results for both the study systems were calculated for a value of $m=5$ and the weighting factors were 
assumed to be unity. These indices have also been calculated for a value of $m=1$, but the results are not reported in this thesis. With $m=1$, the percentage difference in the indices increases. This is due to the masking mentioned earlier, which is more pronounced when the value of $m$ is low.

\section{The RBTS}

All the bus and system indices for this system with $P I_{V}, P I_{M W}$ and $P I_{M W V}$ are exactly the same as the reference case results. The reason why the results are the same is that when $\mathrm{N}=3$, all contingencies for all the $P I$ are calculated using the decoupled load flow method and hence the CPU time with ranking is higher than for the reference case as shown in Table 3.1. The capture ratio is $100.0 \%$. The capture ratio is defined as follows:

$$
\text { Capture Ratio }=\frac{\begin{array}{c}
\text { Number of contingencies that result } \\
\text { in a system problem }
\end{array}}{\begin{array}{c}
\text { Actual number of reference case contingencies } \\
\text { resulting in a system problem }
\end{array}}
$$

The adequacy evaluation of a transmission system in a power network using the ranking

Table 3.1: Comparison of CPU time and capture ratio - RBTS

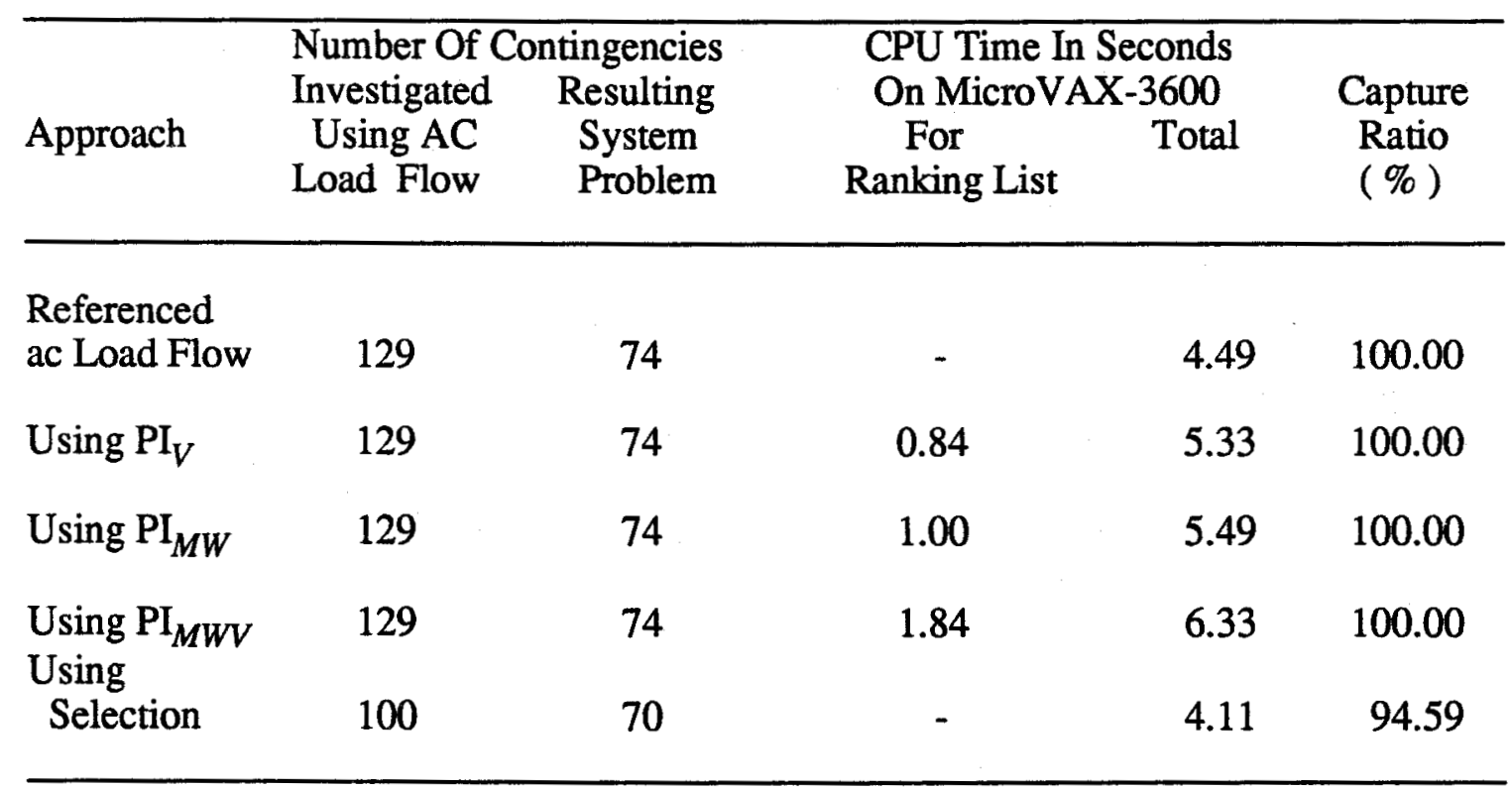


method should consume less computation time than that required without ranking contingencies as this is the prime objective of ranking. For this system, ranking does not offer a good trade-off between the speed of ranking and the subsequent ac solution of the ranked list and the total ac analysis of all the contingencies required for the final solution.

\section{The IEEE-RTS}

The number of contingencies studied, the CPU times and the capture ratios for ranking and selection methods are shown in Table 3.2. The basic bus indices for some selected buses and the system indices for this test system are shown in Table 3.3 through Table 3.5 for the reference ac load flow solution. The percentage differences in the indices for the ranking method with different $P I$ and selection method are also shown in these tables. All the indices for the different $P I$ are lower than their corresponding reference case values. This is to be expected as the number of contingencies examined is

Table 3.2: Comparison of CPU time and capture ratio - IEEE-RTS

\begin{tabular}{lccccc}
\hline & \multicolumn{2}{c}{$\begin{array}{c}\text { Number Of Contingencies } \\
\text { Investigated } \\
\text { Using ac } \\
\text { Load Flow }\end{array}$} & $\begin{array}{c}\text { Resulting } \\
\text { System } \\
\text { Problem }\end{array}$ & $\begin{array}{c}\text { CPU Time In Minutes } \\
\text { On MicroVAX-3600 } \\
\text { For } \\
\text { Ranking List }\end{array}$ & $\begin{array}{c}\text { Total } \\
\text { Capture } \\
\text { Ratio } \\
(\%)\end{array}$ \\
\hline $\begin{array}{l}\text { Referenced } \\
\text { ac Load Flow }\end{array}$ & 9177 & 3184 & - & 26.58 & 100.00 \\
Using PI $V$ & 4031 & 2527 & 1.51 & 19.48 & 79.36 \\
Using PI & & & & & \\
Using PI & 4318 & 2569 & 3.02 & 20.05 & 80.68 \\
$\begin{array}{l}\text { Using } \\
\text { Selection }\end{array}$ & 4682 & 2714 & 4.53 & 23.26 & 85.24 \\
\hline
\end{tabular}

always less than the reference case value. Only 4318,4031 and 4682 contingencies are investigated with performance indices $P I_{M W}, P I_{V}$ and $P I_{M W V}$ respectively which are $47.05 \%, 43.93 \%$ and $51.02 \%$ respectively of the reference case numbers. Out of the 
Table 3.3: Comparison of probability and frequency of failure - IEEE-RTS

\begin{tabular}{|c|c|c|c|c|c|}
\hline \multirow[b]{2}{*}{ Bus } & \multirow{2}{*}{$\begin{array}{l}\text { Referenced } \\
\text { Values }\end{array}$} & \multicolumn{4}{|c|}{--Percentage Difference With- } \\
\hline & & $\mathrm{PI}_{\mathrm{V}}$ & $\mathrm{PI}_{\mathrm{MW}}$ & $\mathrm{PI}_{\mathrm{MWV}}$ & Selection \\
\hline \multicolumn{6}{|c|}{ Probability Of Failure - Bus Indices } \\
\hline 4 & 0.0004496 & -4.14 & -1.18 & -0.02 & 0.00 \\
\hline 5 & 0.0000008 & -12.50 & -25.00 & -12.50 & 0.00 \\
\hline 6 & 0.0013217 & -22.74 & -0.00 & -0.63 & -0.23 \\
\hline 9 & 0.0000237 & -10.13 & -9.28 & 0.00 & -2.95 \\
\hline 14 & 0.0000029 & -10.35 & -13.79 & -6.90 & 0.00 \\
\hline 15 & 0.0000005 & 0.00 & -100.00 & 0.00 & 0.00 \\
\hline 20 & 0.0000025 & -20.00 & -20.00 & -20.00 & -16.00 \\
\hline \multicolumn{6}{|c|}{ Frequency Of Failure - Bus Indices } \\
\hline 4 & 0.4034479 & -1.97 & -1.96 & -0.58 & 0.00 \\
\hline 5 & 0.0011122 & -25.36 & -26.75 & -25.31 & -1.56 \\
\hline 6 & 0.3635413 & -0.04 & -0.01 & -2.68 & -0.04 \\
\hline 9 & 0.0166908 & -4.76 & -15.22 & -0.12 & -7.05 \\
\hline 14 & 0.0035300 & -11.03 & -18.76 & -10.51 & -0.16 \\
\hline 15 & 0.0007741 & -0.45 & -97.68 & -0.40 & -0.14 \\
\hline 20 & 0.0031619 & -23.16 & -23.16 & -23.16 & -23.47 \\
\hline
\end{tabular}

4318 ranked outage events studied using $P I_{M W}$, only 2569 events result in a system problem when they are examined using the fast decoupled ac load flow method. The remaining $19.32 \%$ problem creating contingencies are ranked low by the $P I_{M W}$ and therefore were not included in the subset containing highly ranked contingencies which were studied by ac load flow. Similarly the capture ratio for $P I_{V}$ and $P I_{M W V}$ are $79.37 \%$ and $85.24 \%$ respectively as shown in Table 3.2.

The maximum values in the percentage differences for the four bus indices, the probability of failure, the frequency of failure, the expected load curtailed in MW and the expected energy not supplied in MWh, are $100.00 \%, 97.68 \%, 100.00 \%$, and $100.00 \%$ with $P I_{M W}, 22.74 \%, 92.00 \%, 100.00 \%$ and $100.00 \%$ with $P I_{V}$ and $20.00 \%, 66.36 \%$, $100.00 \%$ and $100.00 \%$ with $P I_{M W V}$ respectively. This is also true for the system indices as seen from Table 3.5. For example, the percentage difference in the value of the severity index is $11.68 \%, 18.98 \%$ and $8.03 \%$ with the different $P I$ shown in Table 3.5. 
Table 3.4: Comparison of load and energy curtailment indices - IEEE-RTS

\begin{tabular}{lcllll}
\hline \multirow{2}{*}{ Bus } & $\begin{array}{c}\text { Referenced } \\
\text { Values }\end{array}$ & $<-3 \mathrm{PI}_{\mathrm{V}}$ & $\mathrm{PI}_{\mathrm{MW}}$ & $\mathrm{PI}_{\mathrm{MWV}}$ & Selection \\
& & & & \\
\hline
\end{tabular}

Expected Load Curtailed (MW) - Bus Indices

4

5

6

9

14

15

20
0.05

0.02

0.13

0.20

0.13

0.03

0.05

$\begin{array}{rr}0.00 & -80.00 \\ -100.00 & -100.00 \\ -7.69 & -15.38 \\ -5.00 & -15.00 \\ -53.85 & -61.54 \\ 0.00 & -100.00 \\ -20.00 & 0.00\end{array}$

$-40.00$

$-15.38$

$-5.00$

$-53.85$

0.00

0.00
0.00

0.00

0.00

$-5.00$

0.00

0.00

$-20.00$

Expected Energy Curtailed (MWh) - Bus Indices

$\begin{array}{rrrrrr}4 & 0.23 & 0.00 & -78.26 & 0.00 & 0.00 \\ 5 & 0.09 & -100.00 & -100.00 & -100.00 & 0.00 \\ 6 & 0.97 & -2.06 & -1.03 & -1.03 & 0.00 \\ 9 & 1.24 & -5.65 & -12.90 & -0.81 & -5.65 \\ 14 & 0.79 & -49.37 & -56.96 & -49.37 & 0.00 \\ 15 & 0.17 & 0.00 & -100.00 & 0.00 & 0.00 \\ 20 & 0.35 & -11.43 & 0.00 & 0.00 & -14.29\end{array}$

The CPU times required to calculate the adequacy indices with different $P I$ are shown in Table 3.2. The total CPU time for ranking with $P I_{V}$ is less than that with $P I_{M W}$. This is due to the fact that in the case of $P I_{V}$, the number of calculations required to calculate $[\Delta \mathrm{V}]$ is less than that required to calculate $[\Delta \delta]$ with $P I_{M W}$, since the dimension of $\left[\mathrm{B}^{\prime \prime}\right]$ is much smaller than the dimension of [B']. The CPU time with $P I_{M W V}$ is the highest, which is obvious, since in this case both $[\Delta \delta]$ and $[\Delta V]$ are calculated using $\left[\mathrm{B}^{\prime}\right]$ and $\left[\mathrm{B}^{\prime \prime}\right]$ respectively.

\subsubsection{Using Selection}

The RBTS

A total of 100 contingencies were found for the RBTS by the selection method out of 129 possible contingencies requiring further investigation using the decoupled ac load 
Table 3.5: Comparison of different system indices - IEEE-RTS

\begin{tabular}{|c|c|c|c|c|c|c|}
\hline & \multirow{2}{*}{$\begin{array}{l}\text { Referenced } \\
\text { Values }\end{array}$} & \multicolumn{4}{|c|}{$<-\cdots$ Percentage Difference With --- } \\
\hline & & & $\mathrm{PI}_{\mathrm{V}}$ & $\mathrm{PI}_{\mathrm{MW}}$ & $\mathrm{PI}_{\mathrm{MWV}}$ & Selection \\
\hline \multicolumn{7}{|c|}{ IEEE Indices } \\
\hline$\overline{\mathrm{BPII}}$ & (MW/MW-Yr) & 0.00036 & -13.89 & -22.22 & -11.11 & -5.56 \\
\hline BPECI & (MWh/MW-Yr) & 0.00228 & -11.40 & -18.86 & -7.90 & -3.51 \\
\hline SI & (System-Min.) & 0.13700 & -11.68 & -18.98 & -8.03 & -3.65 \\
\hline BPSAMC & I(MW/Dist.) & 91.40392 & -0.98 & -5.30 & -3.45 & 2.68 \\
\hline MBPECI & & $0026 * 10^{-3}$ & -11.54 & -19.23 & -7.69 & -3.85 \\
\hline \multicolumn{7}{|c|}{ Average Values for each Load Point per Year } \\
\hline$\overline{\mathrm{ANLC}}$ & & 0.00213 & -10.33 & -15.49 & -1.88 & -8.45 \\
\hline ANVV & & 0.11144 & -0.58 & -0.71 & -0.54 & -0.15 \\
\hline ALC & $(\mathrm{MW})$ & 0.05979 & -13.85 & -22.81 & -9.03 & -4.37 \\
\hline AEC & $(\mathrm{MWh})$ & 0.38177 & -11.51 & -18.57 & -7.56 & -3.61 \\
\hline ANHLC & (Hrs) & 0.01500 & -7.87 & -11.47 & -1.40 & -6.33 \\
\hline
\end{tabular}

flow method. The capture ratio for this system is $94.59 \%$. The differences in the four basic bus adequacy indices are zero for all the buses except bus 3. The percentage differences for the four basic indices are $5.77 \%, 5.77 \% 2.27 \%$ and $2.29 \%$ for bus 3 . The percentage differences in the system indices are very small, and therefore, are not shown separately. The results are shown later in this chapter when combined generation and transmission outages are illustrated. The CPU time is shown in Table 3.1. It can be seen from Table 3.1 that the selection method gives only slightly less accuracy than the reference case and with much less CPU time than ranking. The CPU time with the selection method is 4.11 seconds ( 0.0685 minutes) which is $91.5 \%$ of the reference case CPU time and $74.86 \%$ of the CPU time required with $P I_{M W}$. The ranking method does not provide a good trade-off between CPU times required by ranking and that required by 
the reference case solution, whereas the selection method gives slightly optimistic results with $8.5 \%$ less CPU time than the reference case. Since the CPU time for a small system such as the RBTS is relatively small it is better to examine the contingencies using the decoupled ac load flow method.

\section{The IEEE-RTS}

It can be seen from Table 3.3 through Table 3.5 that the percentage differences in the indices are reduced quite considerably at most of the load points compared to those obtained by the ranking methods and that the proposed method provides results that are closer to the results obtained in the reference case study. The capture ratio with the selection method is $91.8 \%$ which is higher than the capture ratios obtained with ranking methods. It should, however, be noted that the total number of contingencies that were investigated using the fast decoupled ac load flow method is 3754 which is also less than those values obtained with ranking. This outcome resulted in a net saving in the CPU time. The computation time has decreased from 26.58 minutes to 16.67 minutes. The reference case CPU time, is therefore, $60 \%$ more than that required with selection.

It has also been observed that a further improvement in the adequacy indices in the IEEE-RTS can be obtained by including those additional contingencies which cause an angle deviation $|\Delta \delta|$ greater than 30 degrees for any bus. The increase in the computation time is quite nominal but the resulting percentage difference in the indices is less than $1.0 \%$, thus providing results that are quite close to the reference case values.

\subsection{GENERATOR AND GENERATOR + LINE OUTAGES}

Adequacy indices calculated considering only transmission line outages are obviously optimistic due to the fact that the contribution to the adequacy indices from generator and combinations of generator and transmission line outages are very significant. In this study, therefore, the independent outages of generating units up to the fourth contingency level and the independent outages from the combination of transmission lines and generating units up to the third contingency level are also considered in addition to third level independent outages of transmission lines. The 
reasons for considering these levels are discussed in Chapter 2. The number of possible contingencies increases tremendously when these outages are considered in the adequacy evaluation process. For example, the number of possible contingencies of these types are 1551 and 84008 for the RBTS and the IEEE-RTS respectively. The computation time requirement will be very large even for an average size practical power system. In order to reduce the computational burden, the selection approach can be used to limit the number of contingencies studied by the decoupled load flow method. The following selection methods were used for the selection of generating units and generating units combined with transmission line outages.

\subsubsection{Selection Of Generator Outages}

The flow chart for the approximate selection of generating unit outages is shown in Figure 3.4. The main steps of the algorithm are as follows

1. Generator $i$ is in an outage condition.

2. Find the bus number (say $j$ ) of this $i^{\text {th }}$ generator.

3. Calculate the new generation of the jth bus. If new generation is 0.0 then go to step 5, otherwise go to step 4.

4. Calculate the total generation with this ith generator in the outage condition. If the total generation is greater than the total load plus base case system losses, go to step 1 for the next contingency, otherwise go to step 5.

5. Run the contingency by the decoupled ac load flow for further investigation and go to step 1 for the next contingency.

The same algorithm can be used for higher order generator outages.

The number of generator contingencies studied up to the fourth contingency level for the two test systems with and without the selection method are shown in Table 3.6. A significant amount of CPU time can be saved by using the approximate selection method, as can be seen from Table 3.7. This table shows the percentage increase in CPU time and capture ratio of the reference method over those of the selection method using a decoupled ac load flow solution of each contingency. The capture ratio for all contingency levels is $100.0 \%$ for the RBTS. The adequacy indices, therefore, are exactly the same as those of the reference method for this system. The capture ratio for the 


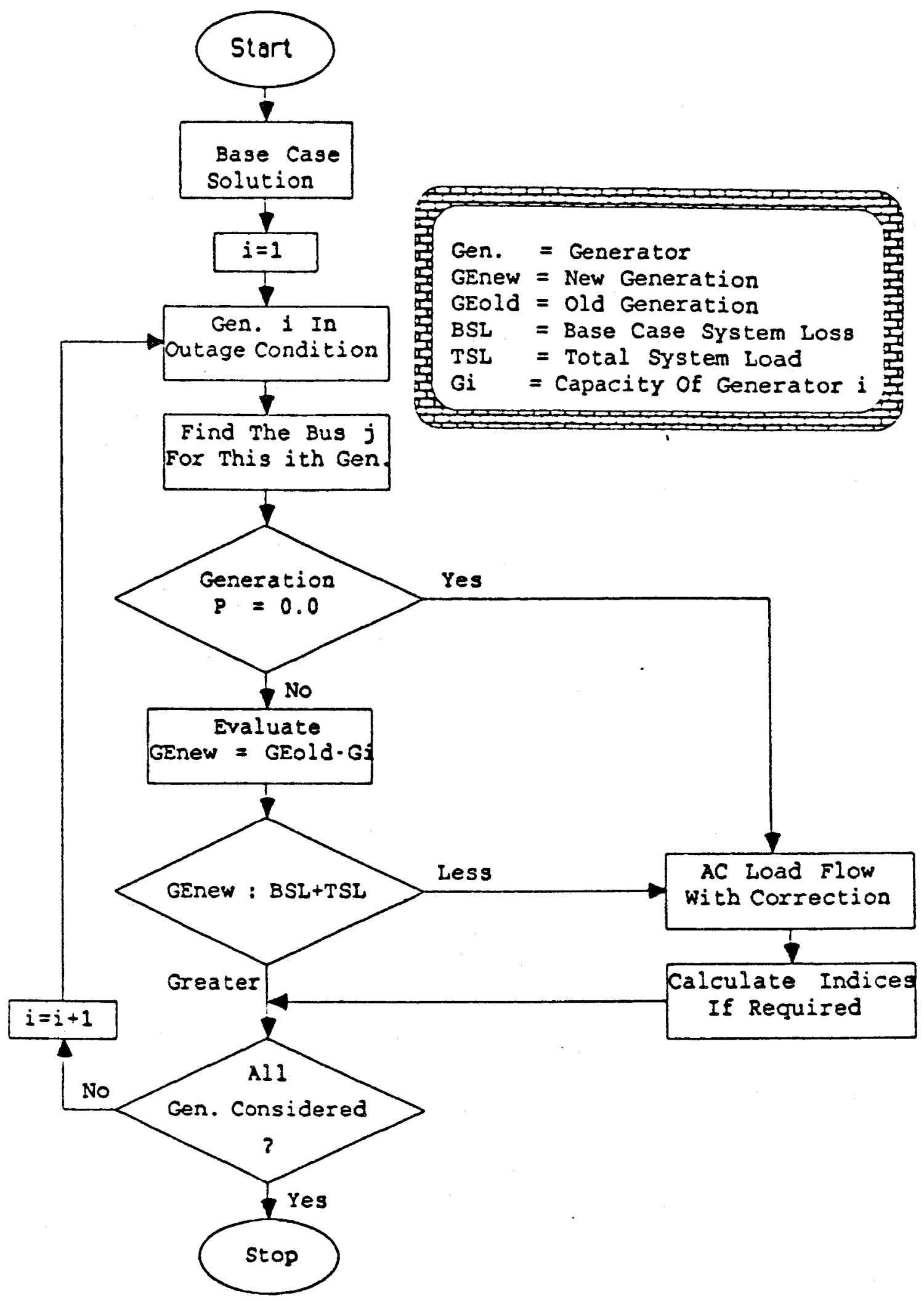

Figure 3.4: Flow chart for the selection of generator contingencies 
Table 3.6: Number of generator contingencies studied by selection

Cont. Contingencies Considered

Levels Using Decoupled ac Load Flow

CPU Time On MicroVAX-3600 Capture

Up To

Reference Selection

Reference Selection

Method Method

Ratio

(\%)

\section{$\underline{\text { RBTS }}$}

First

Second

Third

Fourth

11

66

231

561
2

36

165

490
$0.49 \mathrm{sec}$.

$0.87 \mathrm{sec}$.

$1.24 \mathrm{sec}$.

$2.95 \mathrm{sec}$.
$0.47 \mathrm{sec}$.

$0.75 \mathrm{sec}$.

$0.98 \mathrm{sec}$.

$2.43 \mathrm{sec}$.
100.0

100.0

100.0

100.0

\section{IEEE-RTS}

First

Second

Third

Fourth
32

528

5488

41448
4

150

1844

18048
$1.71 \mathrm{sec}$.

$8.86 \mathrm{sec}$.

$50.76 \mathrm{sec}$.

$5.84 \mathrm{~min}$.
$1.27 \mathrm{sec}$.

$6.39 \mathrm{sec}$.

100.0

$36.23 \mathrm{sec}$.

$4.29 \mathrm{~min}$.

IEEE-RTS is $100.0 \%$ up to the second contingency level. The capture ratio for this system is $99.5 \%$ and $97.8 \%$ for the third and fourth contingency levels respectively. The percentage differences in the adequacy indices are less than $1.0 \%$ at the fourth contingency level for the IEEE-RTS.

\subsubsection{Selection Of Generator And Line Outages}

The same procedure used for the selection of transmission line outages in the previous section can be used in this case by first recognizing the generator unit outages in the study. The generator outage is first considered by calculating a new bus real power injection vector. The new bus phase angle vector is then calculated using the relation

$$
\left[\mathrm{B}^{\prime}\right][\delta]_{\text {new }}=[\mathrm{P}]_{\text {new }}
$$

The $[\mathrm{P}]_{\text {new }}$ matrix is obtained by subtracting those generating units on outage from the original $[\mathrm{P}]$ matrix. The base case lower and upper triangular factors of the system matrix $\left[\mathrm{B}^{\prime}\right]$ are used to find the new angle vector $[\delta]_{\text {new }}$. This $[\delta]_{\text {new }}$ is now considered as 
Table 3.7: Increment in CPU time and capture ratio of the reference method over that of the selection method for generator contingencies

Contingency

Level

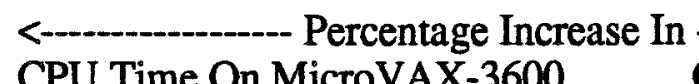

Capture Ratio

RBTS

Up To First

4.26

0.00

Up To Second

16.00

0.00

Up To Third

26.53

0.00

Up To Fourth

21.40

0.00

IEEE-RTS

Up To First

Up To Second

Up To Third

Up To Fourth
34.65

38.65

40.10

36.13
0.00

0.00

0.50

2.20

the base case angle vector and a similar procedure as that used in the transmission line outages selection given in Figure 3.1 is followed in the selection process. The flow chart for this approximate selection is shown in Figure 3.5. The main steps of the algorithm are as follows:

1. Generator $i$ is in an outage condition.

2. Find the bus number (say $\mathrm{j}$ ) of this $\mathrm{i}^{\text {th }}$ generator. If the total new generation is less than the total system load plus base case system losses then investigate this generator outage in combination with all transmission lines outages by the decoupled ac load flow and go to step 1 for the next contingency, otherwise go to step 3.

3. Calculate the new generation of the $j^{\text {th }}$ bus and change the element of real power injection vector [P].

4. Calculate $[\delta]_{\text {new }}$ using Equation 3.8 .

5. Taking this $[\delta]_{\text {new }}$ as $[\delta]_{\text {base }}$ select and run the contingencies using the same flow chart given in Figure 3.1.

The same algorithm can be used for higher order generator and line outages. The number 


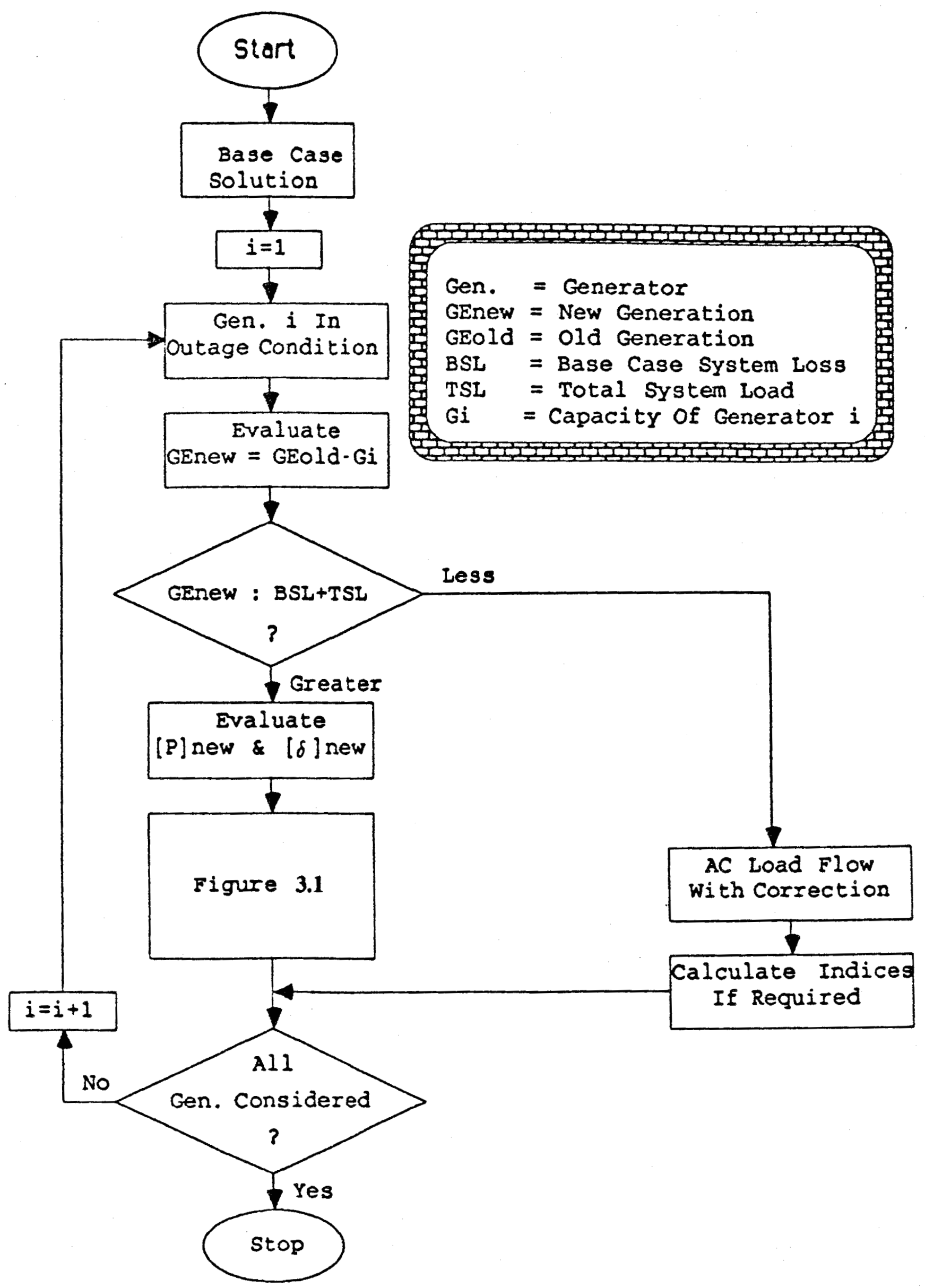

Figure 3.5: Flow chan for the selection of generator+line contingencies 
of combined generator and transmission line contingencies studied by the above procedure is shown in Table 3.8. The CPU times for this study are not shown in the

Table 3.8: Number of generator+line contingencies studied by selection

\begin{tabular}{|c|c|c|c|}
\hline \multirow{2}{*}{$\begin{array}{l}\text { Contingency } \\
\text { Levels Up To }\end{array}$} & $\begin{array}{l}\text { Continge } \\
\text { By Decour }\end{array}$ & $\begin{array}{l}\text { nsidered } \\
\text { oad Flow }\end{array}$ & \multirow{2}{*}{$\begin{array}{l}\% \text { Of Contingencies } \\
\text { Considered By The } \\
\text { Selection Method }\end{array}$} \\
\hline & Reference & Selection & \\
\hline
\end{tabular}

\section{$\underline{\text { RBTS }}$}

First

Second 99

Third

\section{IEEE-RTS}

First

Second

Third
1216

42560
171

12974
14.06

30.48

table, since due to the structure of the program, the independent line and generator contingencies are also considered when combined line and generator contingencies are evaluated. The number of contingencies found by the selection method for further investigation is very small as can be seen from Table 3.8, and hence a significant amount of computer time can be saved with a sacrifice of small percentage errors in the adequacy indices. The adequacy indices are not shown here separately since the indices for the overall study are given in the next subsection.

\subsubsection{Study Results}

In the proposed selection method, contingencies are selected on the basis of line overload and voltage violation. If any of these problems are detected for a particular contingency, that contingency is reexamined using the fast decoupled ac load flow method as noted from the flow charts. During the examination of that particular event, corrective actions such as rescheduling of generation [90] and reactive power injection to 
correct the voltage are first considered before commencing load curtailment. If the corrective actions fail to alleviate the problem(s), load is curtailed using the load curtailment philosophy $[76,93]$. It should be noted that during the examination using a fast decoupled load flow solution, all the failure criteria listed in Table 2.1 are considered. In the selection method, bus isolation is checked before calculating the change in bus angle and voltage magnitude vectors.

The adequacy indices considering all the specified outage combinations were calculated for the two test systems described in Chapter 2. The comparison of CPU times and capture ratios for different contingency levels are shown in Table 3.9 for the two systems. This table also shows percentage comparisons between the two sets of CPU times with and without selection. The quantities inside the brackets are percentage values of CPU time compared to the CPU time of a reference method study without selection. As seen from Table 3.9, the saving in CPU time by selection is more significant for the IEEE-RTS than for the RBTS. The saving in CPU time, for example, is more than 0.3 hour for the IEEE-RTS for the third and fourth contingency levels and only 0.05 minutes for the RBTS at the fourth contingency level. The saving in CPU time is higher for large power networks than for small networks. The numerical values of the different indices together with the percentage variations of the indices are shown in Table 3.10 through Table 3.12 for the RBTS and in Table 3.13 through Table 3.15 for the IEEE-RTS. The percentage differences in all the adequacy indices are very small for both test systems. This can be seen from Table 3.10 through Table 3.15. The percentage difference between the adequacy indices is less than $0.5 \%$ in most cases for both the test systems.

The maximum indices $[2,70]$ obtained by the selection method and the referenced decoupled load flow method are exactly the same for both the test systems. These indices, therefore, are not repeated in this thesis. If the indices of interest are maximum values, then calculation using the selection method is very good even for the small system. All the bus, average and system indices are, however, close to the reference case values and are reasonably accurate. A significant amount of computation time can be saved by using selection with only very small percentage errors in some of the indices. 
Table 3.9: Comparison of contingencies studied for the two test systems

\begin{tabular}{|c|c|c|c|c|c|}
\hline \multirow{2}{*}{$\begin{array}{l}\text { Cont. } \\
\text { Levels } \\
\text { Up To }\end{array}$} & \multicolumn{2}{|c|}{$\begin{array}{l}\text { Contingencies Considered } \\
\text { By Decoupled ac Load Flow }\end{array}$} & \multicolumn{2}{|c|}{$\begin{array}{l}\text { CPU Time On MicroVAX-3600 } \\
\text { In Minutes }\end{array}$} & \multirow{2}{*}{$\begin{array}{l}\text { Capture } \\
\text { Ratio } \\
\text { (\%) }\end{array}$} \\
\hline & Reference & Selection & Reference & Selection & \\
\hline \multicolumn{6}{|l|}{ RBTS } \\
\hline First & 20 & 3 & 0.0115 & $0.0090(78.3 \%)$ & 100.0 \\
\hline Second & 210 & 83 & 0.0405 & $0.0276(68.2 \%)$ & 89.7 \\
\hline Third & 1350 & 807 & 0.2065 & $0.1606(77.8 \%)$ & 94.8 \\
\hline Fourth & 1680 & 1132 & 0.2365 & $0.1833(78.3 \%)$ & 96.4 \\
\hline \multicolumn{6}{|c|}{ IEEE-RTS } \\
\hline First & 70 & 9 & 0.0800 & $0.0444(55.4 \%)$ & 100.0 \\
\hline Second & 2485 & 552 & 2.1675 & $1.1787(54.4 \%)$ & 97.5 \\
\hline Third & 57225 & 18572 & 54.8120 & $36.4410(66.5 \%)$ & 94.5 \\
\hline Fourth & 93185 & 34776 & 59.4052 & $40.1781(67.6 \%)$ & 95.1 \\
\hline
\end{tabular}

Table 3.10: Total probability and frequency of failure - RBTS

\begin{tabular}{|c|c|c|c|c|c|c|}
\hline \multirow[b]{3}{*}{ Bus } & \multicolumn{3}{|c|}{ Probability } & \multicolumn{3}{|c|}{ Frequency } \\
\hline & \multirow{2}{*}{$\begin{array}{l}\text { Reference } \\
\text { Values }\end{array}$} & \multicolumn{2}{|c|}{ With Selection } & \multirow{2}{*}{$\begin{array}{c}\text { Reference } \\
\text { Values }\end{array}$} & \multicolumn{2}{|c|}{ With Selection } \\
\hline & & Values & $\%$ Diff & & Values & $\%$ Diff \\
\hline 2 & 0.0062284 & 0.0062284 & 0.000 & 2.6840122 & 2.6840122 & 0.000 \\
\hline 3 & 0.0087344 & 0.0087276 & -0.078 & 4.2465763 & 4.2341824 & -0.292 \\
\hline 4 & 0.0063303 & 0.0063303 & 0.000 & 2.8416128 & 2.8416128 & 0.000 \\
\hline 5 & 0.0002065 & 0.0002065 & 0.000 & 0.2929301 & 0.2929301 & 0.000 \\
\hline 6 & 0.0011610 & 0.0011610 & 0.000 & 1.1587838 & 1.1587838 & 0.000 \\
\hline
\end{tabular}


Table 3.11: Total expected load and energy curtailment - RBTS

\begin{tabular}{|c|c|c|c|c|c|c|}
\hline \multirow[b]{3}{*}{ Bus } & \multicolumn{3}{|c|}{$\begin{array}{c}\text { Expected Load Curtailed } \\
\text { (MW) }\end{array}$} & \multicolumn{3}{|c|}{$\begin{array}{l}\text { Expected Energy not Supplied } \\
\text { (MWh) }\end{array}$} \\
\hline & \multirow{2}{*}{$\begin{array}{c}\text { Reference } \\
\text { Values }\end{array}$} & \multicolumn{2}{|c|}{ With Selection } & \multirow{2}{*}{$\begin{array}{l}\text { Reference } \\
\text { Values }\end{array}$} & \multicolumn{2}{|c|}{ With Selection } \\
\hline & & Values & $\%$ Diff & & Values & \%Diff \\
\hline 2 & 6.03 & 6.03 & 0.00 & 121.93 & 121.93 & 0.00 \\
\hline 3 & 47.46 & 47.39 & -0.15 & 824.50 & 824.17 & -0.04 \\
\hline 4 & 13.91 & 13.91 & 0.00 & 264.67 & 264.67 & 0.00 \\
\hline 5 & 0.44 & 0.44 & 0.00 & 2.76 & 2.76 & 0.00 \\
\hline 6 & 22.60 & 22.60 & 0.00 & 199.74 & 199.74 & 0.00 \\
\hline
\end{tabular}

\subsection{SUMMARY}

The adequacy indices have been calculated using three different performance indices and are displayed in this chapter. The efficacy and the accuracy of the different ranking methods are compared with the reference case results. It is shown in the case of the RBTS, that the adequacy indices for each of the performance index methods are exactly the same as the reference values. The CPU time, however, is more than the referenced CPU time. Adequacy evaluation using the ranking methods for the IEEERTS, however, requires less computation time than that required without ranking the contingencies. This is to be expected as the number of contingencies examined using the decoupled load flow method are less than the reference values. The accuracy, however, of the adequacy indices is far from being acceptable. This suggests that ranking does not offer a good trade-off between speed and accuracy for the final solution in the IEEERTS.

The inaccuracies in the results for the IEEE-RTS are due to the fact that some of the contingencies which create system problems are ranked low by all the performance indices, whereas some of those which do not produce system problems are ranked high. This chapter also introduced a new approach designated as the selection method which 
Table 3.12: Comparison of system indices - RBTS

\begin{tabular}{llrrr}
\hline & & \multicolumn{2}{c}{$\begin{array}{c}\text { Reference } \\
\text { Values }\end{array}$} & \multicolumn{2}{c}{$\begin{array}{c}\text { Vsing Selection } \\
\text { Values }\end{array}$} & \% Diff \\
\hline IEEE Indices & & & & \\
BPII & (MW/MW-Yr) & 0.48886 & 0.48848 & -0.078 \\
BPECI & (MWh/MW-Yr) & 7.64115 & 7.63932 & -0.024 \\
BPSAMCI & (MW/Dist.) & 15.72272 & 15.74436 & 0.138 \\
SI & (System-Min.) & 458.46900 & 458.359 & -0.024 \\
MBPECI & & 0.00087228 & 0.00087207 & -0.024 \\
Average Values for each Load Point per Year & & \\
ANLC & & 2.24241 & 2.23993 & -0.111 \\
ANVV & & 0.00366 & 0.00366 & 0.000 \\
ALC & (MW) & 18.08772 & 18.07360 & -0.078 \\
AEC & (MWh) & 282.72272 & 282.65503 & -0.024 \\
ANHLC & (Hrs) & 39.69029 & 39.67841 & -0.030 \\
\hline
\end{tabular}

removes the problem of misranking. The selection method selects contingencies on the basis of line overloads and/or voltage violations in a power network.

The selection method for generator, transmission line and combinations of these outages is described in this chapter. It has been shown that the proposed selection method provides better results than the ranking methods. The selection method is faster than the ranking methods and the selection method does not require any extra memory space. Ranking methods, however, need memory locations to store the contingency lists. The memory requirement, depending upon the number of components and the contingency levels up to which the outages are considered, can be quite large for large 
Table 3.13: Total probability and frequency of failure - IEEE-RTS

\begin{tabular}{lccccccc}
\hline & \multicolumn{3}{c}{ Probability } & \multicolumn{3}{c}{ Frequency } \\
Bus & $\begin{array}{c}\text { Reference } \\
\text { Values }\end{array}$ & $\begin{array}{c}\text { With Selection } \\
\text { Values }\end{array}$ & \%Diff & $\begin{array}{c}<-1 \text { Reference } \\
\text { Values }\end{array}$ & $\begin{array}{r}\text { With Selection } \\
\text { Values }\end{array}$ \\
& & & & & & \\
\hline & & & & & & \\
1 & 0.0221474 & 0.0220945 & -0.239 & 16.1407547 & 16.0923023 & -0.300 \\
2 & 0.0407428 & 0.0406371 & -0.259 & 29.3973236 & 29.3004093 & -0.330 \\
3 & 0.0244430 & 0.0243823 & -0.248 & 17.6051254 & 17.5452461 & -0.340 \\
4 & 0.0226639 & 0.0226109 & -0.234 & 16.7103462 & 16.6617451 & -0.291 \\
5 & 0.0223076 & 0.0222541 & -0.240 & 16.2549496 & 16.2055073 & -0.304 \\
6 & 0.0234258 & 0.0233705 & -0.236 & 17.0112495 & 16.9611969 & -0.294 \\
7 & 0.0168814 & 0.0167405 & -0.835 & 12.5238333 & 12.3933992 & -1.041 \\
8 & 0.0173257 & 0.0170136 & -1.801 & 12.8308020 & 12.5562935 & -2.139 \\
9 & 0.0036907 & 0.0036883 & -0.065 & 2.4428589 & 2.4394534 & -0.139 \\
10 & 0.0035938 & 0.0035921 & -0.047 & 2.3835158 & 2.3811319 & -0.100 \\
13 & 0.0699188 & 0.0697588 & -0.229 & 44.6458549 & 44.4970818 & -0.333 \\
14 & 0.0097601 & 0.0097583 & -0.018 & 6.8995028 & 6.8969927 & -0.036 \\
15 & 0.0578149 & 0.0577665 & -0.084 & 36.1420097 & 36.0961838 & -0.127 \\
16 & 0.0255685 & 0.0255200 & -0.190 & 17.9778633 & 17.9319954 & -0.255 \\
18 & 0.0846247 & 0.0846247 & 0.000 & 52.2802353 & 52.2802124 & 0.000 \\
19 & 0.0119094 & 0.0119083 & -0.009 & 8.2971764 & 8.2954683 & -0.021 \\
20 & 0.0452424 & 0.0452151 & -0.060 & 29.1360416 & 29.1082706 & -0.095 \\
& & & & & & \\
\hline
\end{tabular}

power networks. The selection method is simple and flexible and can be used to examine both continuity and quality of power supply for transmission line and combined generator and transmission line outages. It can be easily implemented with any computer program package developed for studying the adequacy of a power network.

The most significant parameters in a bulk power adequacy assessment are the load point and overall system 'load curtailment indices'. Many utilities have difficulty in interpreting the calculated load curtailment indices as the existing models do not always consider realistic and practical operating conditions. Some of these concerns regarding the existing models were discussed in a recent report sponsored by EPRI [69]. This report indicated that actual load curtailment is not the primary concern when justifying the need for bulk power system facilities. The utility survey also indicated that in composite system planning, system indices may be more important than load curtailment 
Table 3.14: Total expected load and energy curtailment - IEEE-RTS

\begin{tabular}{|c|c|c|c|c|c|c|}
\hline \multirow[b]{3}{*}{ Bus } & \multicolumn{3}{|c|}{$\begin{array}{l}\text { Expected Load Curtailed } \\
\text { (MW) }\end{array}$} & \multicolumn{3}{|c|}{$\begin{array}{l}\text { Expected Energy not Supplied } \\
\text { (MWh) }\end{array}$} \\
\hline & \multirow{2}{*}{$\begin{array}{l}\text { Reference } \\
\text { Values }\end{array}$} & \multicolumn{2}{|c|}{ With Selection } & \multirow{2}{*}{$\begin{array}{l}\text { Reference } \\
\text { Values }\end{array}$} & \multicolumn{2}{|c|}{ With Selection } \\
\hline & & Values & \%Diff & & Values & $\%$ Diff \\
\hline 1 & 162.80 & 162.74 & -0.04 & 1998.25 & 1997.65 & -0.03 \\
\hline 2 & 299.16 & 299.03 & -0.04 & 3670.04 & 3668.86 & -0.03 \\
\hline 3 & 364.87 & 364.70 & -0.05 & 4543.25 & 4541.88 & -0.03 \\
\hline 4 & 168.62 & 168.56 & -0.04 & 2086.64 & 2086.14 & -0.02 \\
\hline 5 & 139.90 & 139.84 & -0.04 & 1741.78 & 1741.29 & -0.03 \\
\hline 6 & 309.95 & 309.83 & -0.04 & 3863.04 & 3861.95 & -0.03 \\
\hline 7 & 151.48 & 151.22 & -0.17 & 1818.37 & 1816.17 & -0.12 \\
\hline 8 & 313.44 & 313.00 & -0.14 & 3855.53 & 3852.00 & -0.09 \\
\hline 9 & 51.05 & 50.95 & -0.20 & 619.87 & 619.27 & -0.10 \\
\hline 10 & 51.67 & 51.58 & -0.17 & 639.10 & 638.55 & -0.09 \\
\hline 13 & 1722.03 & 1721.26 & -0.04 & 23258.48 & 23252.25 & -0.03 \\
\hline 14 & 155.31 & 155.00 & -0.20 & 1843.39 & 1841.41 & -0.11 \\
\hline 15 & 1942.06 & 1941.99 & 0.00 & 27895.06 & 27894.41 & 0.00 \\
\hline 16 & 202.04 & 202.02 & -0.01 & 2452.25 & 2452.05 & -0.01 \\
\hline 18 & 3366.20 & 3366.20 & 0.00 & 50921.38 & 50921.36 & 0.00 \\
\hline 19 & 172.32 & 172.28 & -0.02 & 2077.29 & 2077.02 & -0.01 \\
\hline 20 & 837.85 & 837.80 & -0.01 & 11681.20 & 11680.83 & 0.00 \\
\hline
\end{tabular}

indices and that system security is an important issue. In the following chapters, the reliability assessment of composite systems will be examined from a security point of view. The composite generation and transmission system is divided into different operating states specified in terms of the degree to which adequacy and security constraints are satisfied. Probabilistic indices are provided to assess these states. The selection method described in this chapter are an integral part of this technique and is used to detect problem creating contingencies. 
Table 3.15: System indices for the IEEE-RTS

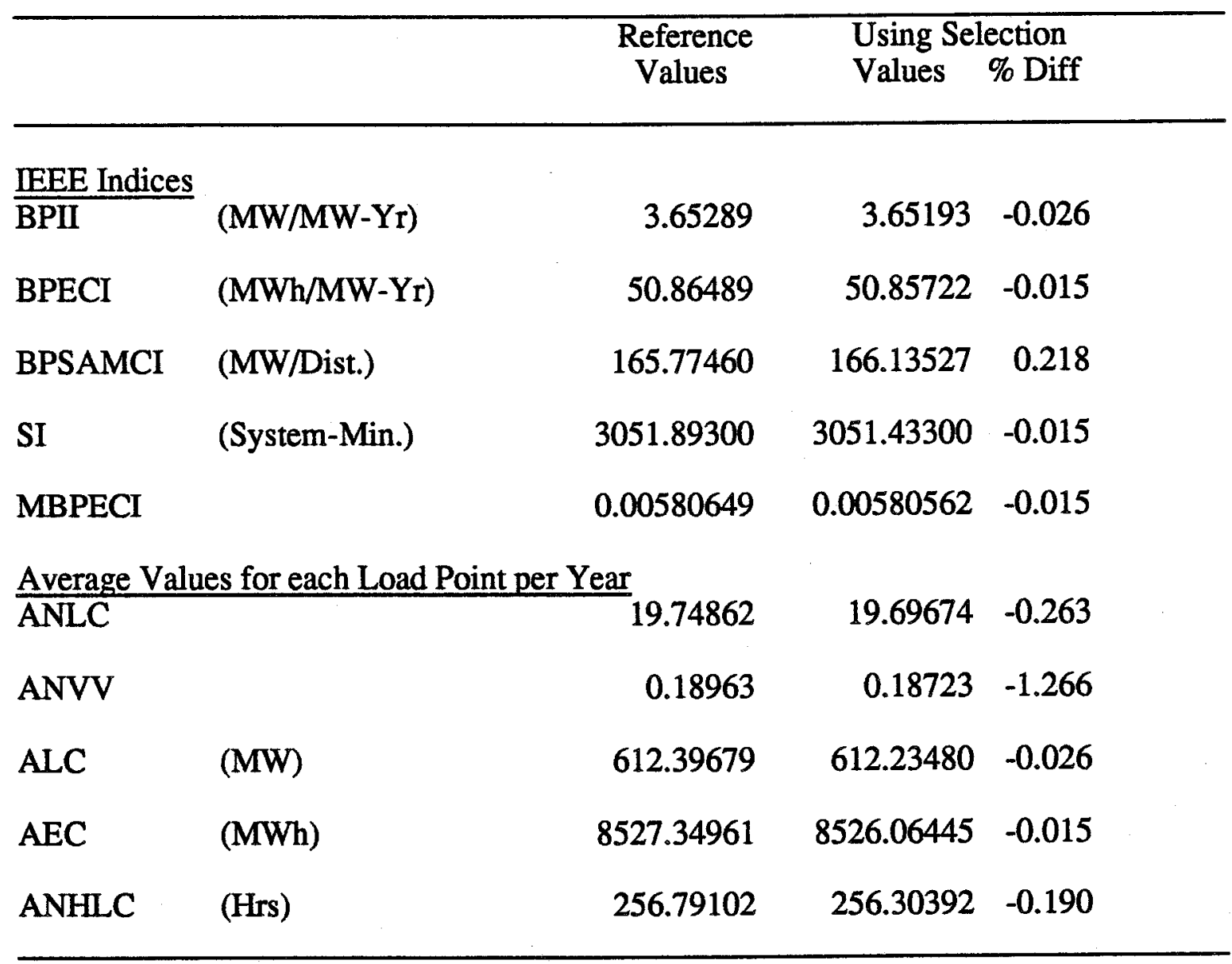




\section{PROBABILISTIC SECURITY ASSESSMENT OF A COMPOSITE POWER SYSTEM}

\subsection{INTRODUCTION}

Reliability evaluation of bulk power systems has been a major system planning concern for many years. The presently available techniques together with important related concerns were recently addressed in an IEEE Power Engineering Society tutorial [67] and were summarized in Reference [65]. Power system reliability can be categorized into the domains of adequacy and security and as noted in Reference [67], virtually all of the available methods for the quantitative reliability evaluation of a bulk power system are in the adequacy domain. System adequacy is defined as the ability of the system to supply its load taking into consideration transmission constraints and scheduled and unscheduled outages of generators and transmission facilities. System security is defined as the ability of the power system to withstand disturbances arising from faults or unscheduled removal of bulk power supply equipment. Adequacy assessment, therefore, is the steady state post outage analysis of the bulk power system while security assessment involving dynamic condition analysis. The most significant quantitative indices in composite system adequacy assessment are those which relate to load curtailment at both the individual bus and overall system levels. These indices can be extended to recognize additional factors such as customer interruption costs and reliability cost/reliability worth considerations $[2,67,97]$. There are a number of different computer programs available for composite system adequacy evaluation. These programs are briefly described in Reference [68], including a list of the calculated indices and the factors involved in the assessment. Many utilities have difficulty in interpreting the expected load curtailment indices as the existing models are based on adequacy analysis and in many cases do not consider realistic operating conditions. These concerns were expressed in response to a survey conducted as part of an EPRI project 
and are summarized in the project report [69]. This survey also indicated that security considerations are important issues in composite system reliability evaluation. In response to the stated utility concerns, a framework for incorporating security considerations was also proposed in the project report.

This framework has been examined and extended in this chapter. A new set of system reliability indices are proposed and developed which include system security considerations. It is believed that these indices will prove to be intuitively appealing to both system planners and operators and will form the basis of new system reliability criteria.

The Sherman-Morrison correction formula is generally used in conventional HLII analysis to modify the base case solution vectors instead of rebuilding and refactorizing the system admittance matrices. Multiple line outages are represented by applying the formula recursively, and updating the solution vector at each step. An alternative efficient approach, in conjunction with the selection method, which avoids the recursive use of the Sherman-Morrison correction formula for multiple lines outages is also presented in this chapter.

\subsection{SECURITY CONSIDERATIONS}

\subsubsection{System Model Including Security Considerations}

In order to recognize security considerations in the evaluation of a composite system, the total power network can be divided into several operating states in terms of the degree to which adequacy and security constraints are satisfied. Figure 4.1 shows a probable classification of a system $[69,72]$. The subsequent quotations are taken from the EPRI report [69].

The normal state is defined as [69]

"In the normal state, all equipment and operation constraints are within limits, including that the generation is adequate to supply the load (total demand), with no equipment overloaded. In the normal 


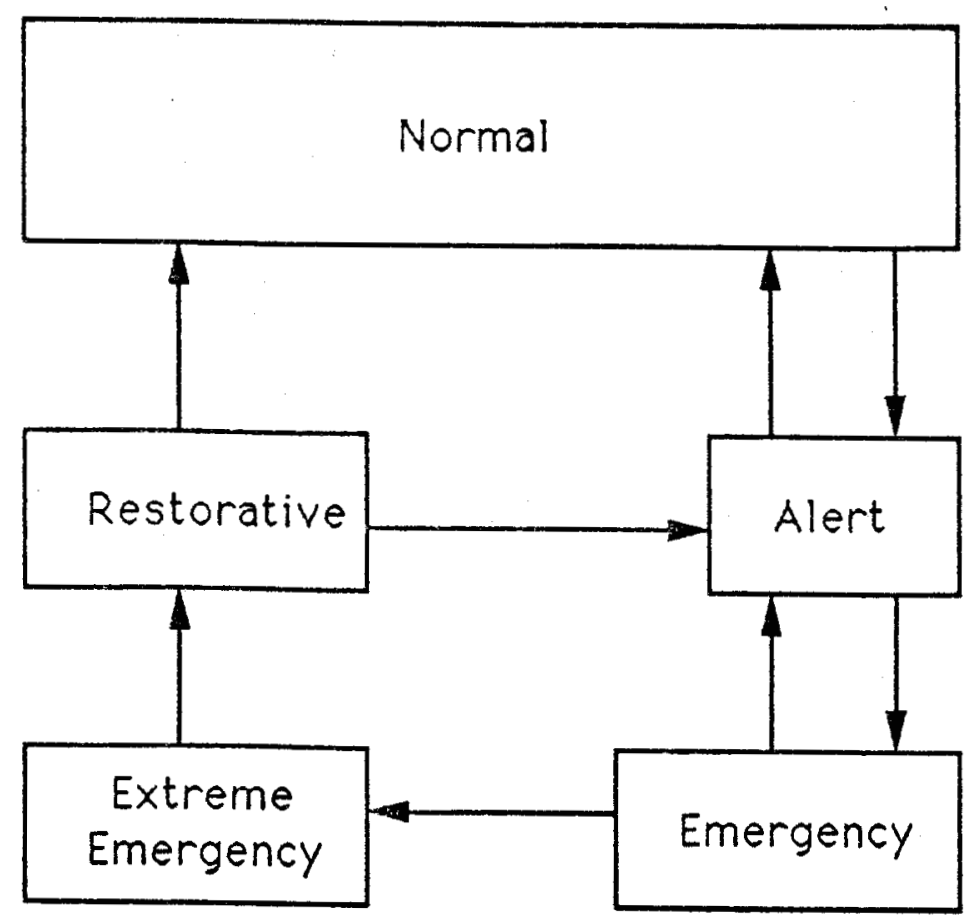

Figure 4.1: System operating states

state, there is sufficient margin such that the loss of any element, specified by some criteria, will not result in a limit being violated. The particular criteria, such as all single elements, will depend on the planning and operating philosophy of a particular utility".

From the definition it is clear that the system is both adequate and secure in the normal state.

The alert state is defined as [69]

"If a system enters a condition where the loss of some element covered by the operating criteria will result in a current or voltage violation, then the system is in the alert state. The alert state is similar to the normal state in that all constraints are satisfied, but there is no longer sufficient margin to withstand an outage 
(disturbance). The system can enter the alert state by the outage of equipment, by a change in generation schedule, or a growth in the system load".

In the alert state the system no longer has sufficient margin to satisfy the security constraints.

The emergency state is defined as [69]

"If a contingency occurs or the generation and load changes before corrective action can be (or is) taken, the system will enter the emergency state. No load is curtailed in the emergency state, but equipment or operating constraints have been violated. If control measures are not taken in time to restore the system to the alert state, the system will transfer from the emergency state to the extreme emergency state".

In this state both adequacy and security constraints are violated. This is a temporary state which requires operator action because equipment operating constraints have been violated. The first objective will be to remove the equipment operating constraints without load curtailment, by such means as phase shifter adjustment, redispatch, or startup of additional generation. If successful, this could lead to the alert state, where further actions would still be necessary to achieve the normal state. Such actions could include voltage reduction. On the other hand, once the alert state is reached, it may be decided to take no further control action as described previously.

The extreme emergency state is defined as follows [69]

"In the extreme emergency state, the equipment and operating constraints are violated and load is not supplied."

In this state, load has to be curtailed in a specific manner in order to return from this state to another state. 


\subsubsection{Modified System Model}

One difficulty with the model shown in Figure 4.1 is that there is no connection shown between the normal and the extreme emergency states. Connection is only through the alert and emergency states. The system state can go directly from the normal to the extreme emergency state depending on the outage and the system. This transition may or may not be possible in certain systems. For example, the test system RBTS [91] defined in Chapter 2 has $185 \mathrm{MW}$ of load at the time of system peak demand. The total generation capacity available for this system is $240 \mathrm{MW}$. If two large generating units $(40 \mathrm{MW}+40 \mathrm{MW})$ are removed from service at the time of system peak demand, the system state will go directly from the normal state to the extreme emergency state presuming that the system was in the normal state prior to the event. If one of the large units is out of service, the system will be in the alert state, the loss of another large unit will cause the system to go to the extreme emergency state. In order to overcome the above difficulty and to keep the definitions given in Reference [69], the system operating states have been modified as shown in Figure 4.2. After defining system failure and specifically identifying the conditions of interest for the study, there are several reliability measures that can be used to provide indices for these conditions. The composite system reliability assessment including security considerations in the present and following chapters involve:

1. identifying events that lead to the alert, emergency, etc. states as shown in Figure 4.2,

2. calculating reliability indices for each of the above states. The basic indices are:
a. state probabilities
b. state frequencies

A direct approach to calculate the reliability indices for the model of Figure 4.2 involves a full ac load flow analysis for each of the contingencies up to a certain outage level or contingencies of interest in order to determine the effect of unscheduled or unexpected disturbances or contingencies on the power system. The main objective of this simulation is to determine: 


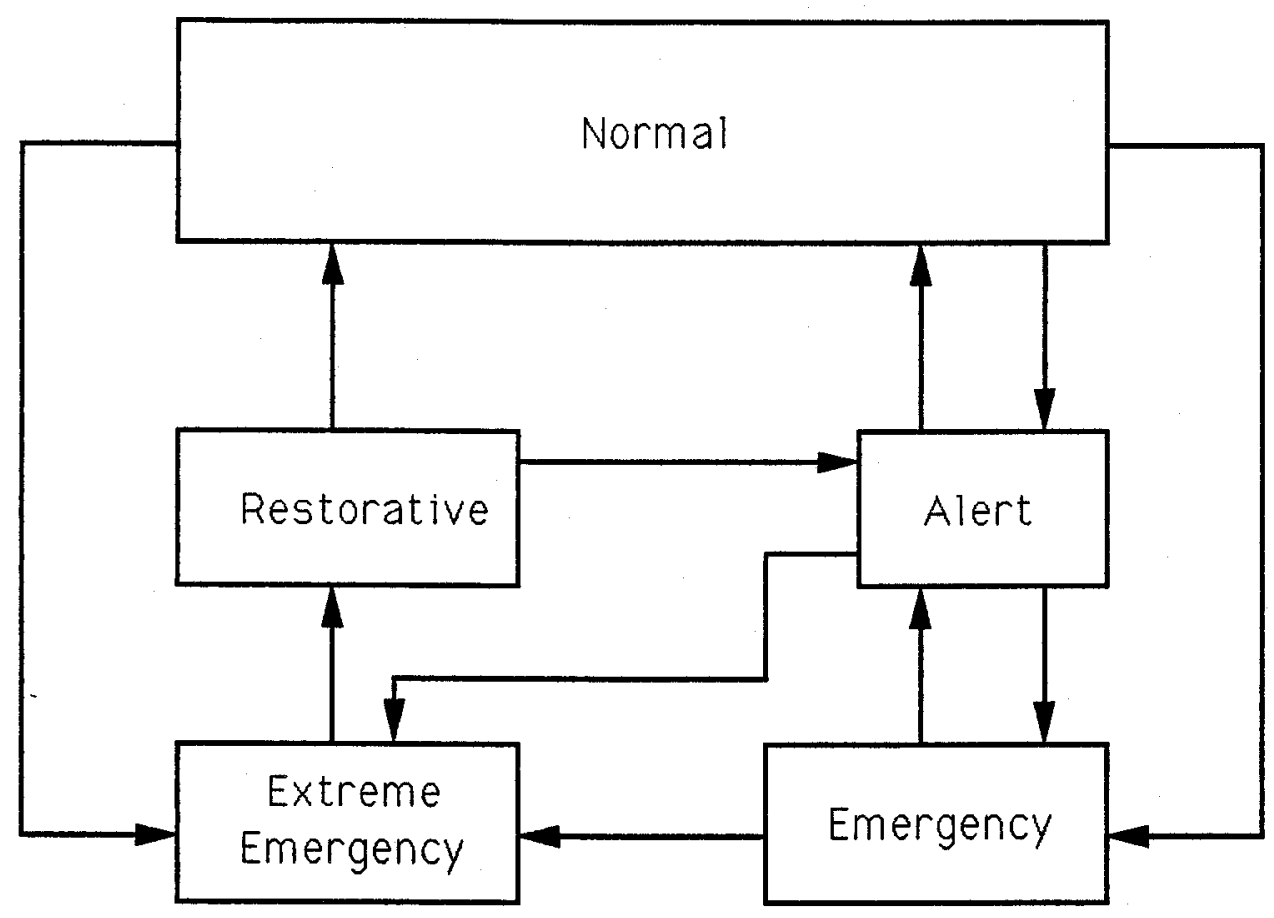

Figure 4.2: Modified diagram for system operating states

1. those contingencies that cause limit violation(s) and

2. the associated limit violation(s).

An exhaustive power flow simulation is not computationally feasible because of the need to consider higher level outages [41] especially for a large power network. In order to reduce the computation time in the security/adequacy analysis, selection or sensitivity based ranking methods with consecutive success cutoff criterion can be used. Sensitivity based ranking methods $[43,44,45,46,48]$ do not identify or solve for specific system conditions. Rather, they quantify the severity of each outage by explicitly calculating a scalar value called a "performance index" by which all contingencies can be ranked as explained in Chapter 3. The methods are not completely reliable since they are prone to 'masking errors'. Specifically, a contingency with a few severe violations can be ranked equally with one with many minor violations or even worse, with one without violations. The screening methods $[54,58,59,62]$, though more demanding in computer resources, permit the identification of actual violations/major shifts and, therefore avoid masking 
errors. The main objective of incorporating security considerations in power system reliability evaluation is to determine which contingencies cause component limit violations and/or major system shifts and to determine the severity of the contingencies. It is reasonable to consider violations of branch flow limits, bus voltage limits and generator var limits. The selection methods $[41,82]$ are therefore used in the studies reported in this thesis to detect the problem created contingencies for line, generator and combined line and generator outages. The corrective actions that have been considered with the selection methods and corresponding mathematical models are presented in the following sections and in Chapter 5.

\subsubsection{Security Constraints}

The security constraints are the operating limits which have to be satisfied for the normal operation of the power network. These constraints depend mainly on the purpose behind the study and the method used in the study. The basic security constraints in order to operate the system within an acceptable security domain are:

\section{Voltage magnitude constraints:}

operating limits are imposed on the voltage magnitude of $P Q$ buses i.e.

$$
\mathrm{V}^{\mathrm{m}} \leq \mathrm{V} \leq \mathrm{V}^{\mathrm{M}}
$$

define the region inside the limits by $R_{v}$ i.e.

$$
\mathrm{R}_{\mathrm{v}}:=\left\{\mathrm{V}: \mathrm{V}^{\mathrm{m}} \leq \mathrm{V} \leq \mathrm{V}^{\mathrm{M}}\right\}
$$

where $\mathrm{V}^{\mathrm{m}}$ and $\mathrm{V}^{\mathrm{M}}$ represent the minimum and the maximum voltage limits.

\section{Line flow constraints:}

the thermal operating limits of the transmission line and transformer limits the amount of current flow through them. These can be expressed as

$$
\left|\delta_{\mathrm{k}}-\delta_{\mathrm{i}}\right| \leq \theta_{\mathrm{j}}
$$

where $\mathrm{j}$ is the branch connecting the buses $\mathrm{k}$ and $\mathrm{i}$. The corresponding region for all lines 
is

$$
R_{\theta}:=\{\delta:-\Theta \leq \delta \leq \Theta\}
$$

Real power generation constraints:

the real power constraints at slack bus or PV buses are

$$
P_{k}{ }^{m} \leq P_{k} \leq P_{k}^{M}
$$

where $P_{k}{ }^{m}$ and $P_{k}{ }^{M}$ represent the minimum and the maximum power generation at bus $k$. The corresponding region is defined by $R_{p}$.

There may be other constraints which are system specific, such as angle spread across the system due to stability limits or reactive power generation constraints. The approach presented provides a basic framework within which any particular constraint can be added to the total constraint set.

\section{Security constraint set}

The security constraint set can be constructed using any one or using a combination of the above constraints. In the results presented in the studies, the security constraint set is formed by combining all of the above three constraints. Therefore, the power system will be considered to be operating within limits when there exists a solution in $(\mathrm{V}, \delta)$-space which satisfies the following relation:

$$
\mathbf{R}:=\mathbf{R}_{\mathbf{v}} \cap \mathbf{R}_{\theta} \cap \mathbf{R}_{\mathbf{p}}
$$

Two other security constraint sets are also formed for the purpose of comparing results. These sets are defined as:

Set 1: $\mathbf{R}_{\mathbf{1}}:=\mathbf{R}_{\boldsymbol{\theta}} \cap \mathbf{R}_{\mathbf{p}}$ overload problem

Set 2: $R_{2}:=R_{v} \quad$ voltage violation 


\subsection{MATHEMATICAL MODEL}

\subsubsection{Selection Method}

The selection methods [82] used for the calculation of the probabilistic indices for different operating states are based on the two decoupled equations [60] given by

$$
\begin{aligned}
{[\mathrm{P}] } & \approx[\mathrm{P} / \mathrm{V}]=\left[\mathrm{B}^{\prime}\right][\delta] \\
\text { and }[\mathrm{Q}] & \approx[\mathrm{Q} / \mathrm{V}]=\left[\mathrm{B}^{\prime \prime}\right][\mathrm{V}]
\end{aligned}
$$

The selection methods as explained in detail in Chapter 3, commences with the the decoupled load flow solution and updates the changes in bus voltage magnitudes and angles using the equations described in the next section, followed by the limits check for the respective constraint set for a contingency without solving the load flow equations. The changes in the vectors can be computed by the recursive use of the ShermanMorrison correction formula [80] for multiple line outages. The recursive use is avoided for multiple line outages with the formula derived below. This will result in a reduction in computation time.

\subsubsection{Method For Calculating Changes In Voltage And Angle Vectors}

Instead of rebuilding and refactorizing the system admittance matrices for each line outage contingency, the Sherman-Morrison correction formula is normally used to adjust the base case solution to effectively represent the lines outages. The algorithm is applicable to both $\mathrm{dc}$ and decoupled load flows. Multiple lines outages are represented by applying the formula recursively, and updating the solution vector at each step. The computation time therefore increases for multiple lines outage cases due to the recursive use of the Sherman-Morrison correction formula. It has been shown in Reference [47] that for third level line outages, rebuilding and refactorization of the system matrices takes less computation time than the recursive use of the Sherman-Morrison correction formula with decoupled load flow equations. The computation time for second level line outages is also comparable. The method described below avoids the recursive use of the Sherman-Morrison correction formula for any level of outages. 
Let either Equation 4.9 or 4.10 be represented in the base case situation by the following equation

$$
[R]=[B][E] \text {. }
$$

It is clear from Equation 4.11 that for a fixed set of active power injections [P] or reactive power injections $[\mathrm{Q}]$, both $[\mathrm{B}]$ matrix and $[\mathrm{E}]$ vector will change from their base-case values if a line or lines are removed. Let the change be represented by $[\Delta \mathrm{B}]$ and $[\Delta \mathrm{E}]$ such that

$$
\begin{aligned}
& {[R] }=\{[B]+[\Delta B]\}\{[E]+[\Delta E]\} \\
&=[B][E]+[B][\Delta E]+[\Delta B]\{[E]+[\Delta E]\} \\
& \text { or, }\{[B]+[\Delta B]\}[\Delta E]=-[\Delta B][E]
\end{aligned}
$$

The changes in the $[E]$ vector i.e. $[\Delta E]$ due to network changes can be calculated from Equation 4.12 using several different methods. One of the widely used approaches is the Sherman-Morrison correction formula [80] noted earlier. In this security analysis, higher level contingencies have to be considered in order to determine which contingency belongs to which operating state according to the definition of different operating states. It is, therefore, considerably faster to use the inverse of $[B]$. This assumes that there is sufficient storage to retain the upper or lower triangular parts of $[B]^{-1}$. Tinney and Powell [95] pointed out that the inverse of a symmetric matrix may be computed very efficiently by back substitution using the triangular factors. The process is of the order $\mathbf{n}^{2}$, whereas conventional inversion is of the order of $n^{3}$. The back substitution method requires about $2 n^{2}$ multiplications.

Given the upper or lower triangular part of $[B]^{-1}$, Equation 4.12 can be written as

$$
\begin{aligned}
\left\{I+B^{-1} \Delta B\right\} \Delta E & =-B^{-1} \Delta B E \\
\text { or, } \quad\{I+X \Delta B\} \Delta E & =-X \Delta B E
\end{aligned}
$$

where $\mathbf{X}=\mathbf{B}^{-1}$ and $\mathbf{I}$ is an identity matrix. Matrices or vectors are written using capital bold letters without matrix notation for simplicity. For the independent outage of lines 1 and 2 connecting buses ' $p$ - $q$ ' and ' $m$ - $n$ ' respectively, the above equation will be 
$\left\{I+X\left(b_{1} M_{1} M_{1}{ }^{t}+b_{2} M_{2} M_{2}{ }^{t}\right)\right\} \Delta E=-X\left\{b_{1} M_{1} M_{1}{ }^{t}+b_{2} M_{2} M_{2}^{t}\right\} E$

or,

$$
\left\{I+b_{1} Z_{1} M_{1}^{t}+b_{2} Z_{2} M_{2}^{t}\right\} \Delta E=-\left\{b_{1} Z_{1} M_{1}{ }^{t}+b_{2} Z_{2} M_{2}{ }^{t}\right\} E
$$

where:
M's $=(\mathrm{Nx} 1)$ dimensional column vector with all zeros except the two elements corresponding to the buses which are 1 and -1 respectively
$\mathbf{Z}=\mathbf{X} * \mathbf{M}$ is a $(\mathrm{Nx} 1)$ dimensional column vector.
b's = the negative value of line susceptances.

The terms of Equation 4.13 can be calculated as follows:

$$
\mathbf{I} \Delta \mathbf{E}=\left[\begin{array}{c}
\Delta \mathrm{e}_{1} \\
\Delta \mathrm{e}_{2} \\
\Delta \mathrm{e}_{3} \\
\vdots \\
\vdots \\
\Delta \mathrm{e}_{n}
\end{array}\right]
$$

and

$$
\begin{aligned}
b_{1} Z_{1} M_{1}{ }^{l} \Delta E & =b_{1}\left(\Delta e_{p}-\Delta e_{q}\right) Z_{1} \\
& =b_{1}\left(\Delta e_{p}-\Delta e_{q}\right)\left[\begin{array}{c}
x_{1 p}-x_{1 q} \\
x_{2 p}-x_{2 q} \\
\vdots \\
\vdots \\
\vdots \\
x_{n p}-x_{n q}
\end{array}\right]
\end{aligned}
$$

Similar expressions for other terms of Equation 4.13 can be derived. From Equation 4.13 through Equation 4.15, it is clear that all rows of Equation 4.13 have five elements at the left hand side except for the rows $p, q, m$ and $n$ corresponding to buses connected by lines 1 and 2. Each of these four rows have four elements on the left hand side. Four equations can be derived for these four rows which in matrix form are as follows: 


$$
\begin{aligned}
& {\left[\begin{array}{cccc}
1+c_{1} & -c_{1} & d_{1} & -d_{1} \\
c_{2} & 1-c_{2} & d_{2} & -d_{2} \\
c_{3} & -c_{3} & 1+d_{3} & -d_{3} \\
c_{4} & -c_{4} & d_{4} & 1-d_{4}
\end{array}\right]\left[\begin{array}{c}
\Delta e_{p} \\
\Delta e_{q} \\
\Delta e_{m} \\
\Delta e_{n}
\end{array}\right]=-\left[\begin{array}{c}
r_{1} \\
r_{2} \\
r_{3} \\
r_{4}
\end{array}\right]} \\
& \text { where: } \\
& \mathrm{c}_{1}=\mathrm{b}_{1}\left(\mathrm{x}_{p p}-\mathrm{x}_{p q}\right) \quad \mathrm{d}_{1}=\mathrm{b}_{2}\left(\mathrm{x}_{p m}-\mathrm{x}_{p n}\right) \quad \mathrm{r}_{1}=\mathrm{k}_{1} \mathrm{c}_{1}+\mathrm{k}_{2} \mathrm{~d}_{1} \\
& \mathrm{c}_{2}=\mathrm{b}_{1}\left(\mathrm{x}_{q p}-\mathrm{x}_{q q}\right) \quad \mathrm{d}_{2}=\mathrm{b}_{2}\left(\mathrm{x}_{q m}-\mathrm{x}_{q n}\right) \quad \mathrm{r}_{2}=\mathrm{k}_{1} \mathrm{c}_{2}+\mathrm{k}_{2} \mathrm{~d}_{2} \\
& c_{3}=b_{1}\left(x_{m p}-x_{m q}\right) \quad d_{3}=b_{2}\left(x_{m m}-x_{m n}\right) \quad r_{3}=k_{1} c_{3}+k_{2} d_{3} \\
& \mathrm{c}_{4}=\mathrm{b}_{1}\left(\mathrm{x}_{n p}-\mathrm{x}_{n q}\right) \quad \mathrm{d}_{4}=\mathrm{b}_{2}\left(\mathrm{x}_{n m}-\mathrm{x}_{n n}\right) \quad \mathrm{r}_{4}=\mathrm{k}_{1} \mathrm{c}_{4}+\mathrm{k}_{2} \mathrm{~d}_{4} \\
& \mathrm{k}_{1}=e_{p}-e_{q} \text { and } \mathrm{k}_{2}=e_{m}-e_{n}
\end{aligned}
$$

The four values, namely $\Delta e_{p}, \Delta e_{q}, \Delta e_{m}$ and $\Delta e_{n}$ can be easily calculated from Equation 4.16. The other values of $\Delta \mathbf{E}$ can be calculated from these known four values using the following relation.

where:

$$
\Delta E=E^{1}-b_{1}\left(\Delta e_{p}-\Delta e_{q}\right) Z_{1}-b_{2}\left(\Delta e_{m}-\Delta e_{n}\right) Z_{2}
$$

$$
E^{1}=-b_{1}\left(e_{p}-e_{q}\right) Z_{1}-b_{2}\left(e_{m}-e_{n}\right) z_{2}
$$

Thus, the above equations become as:

$$
\Delta E=-b_{1}\left(e_{p}-e_{q}+\Delta e_{p}-\Delta e_{q}\right) Z_{1}-b_{2}\left(e_{m}-e_{n}+\Delta e_{m}-\Delta e_{n}\right) Z_{2}
$$

Equation 4.17 gives the correction vector for the second level outages. This equation is simple and straight-forward. In order to use Equation 4.17 it is necessary to invert a matrix of dimension $(4 \times 4)$ given as Equation 4.16. The maximum dimension for $n$th level outages for this matrix is $2 \mathrm{n} \times 2 \mathrm{n}$. The dimension depends on the type of buses connected by the lines on outage. For example, in order to calculate $[\Delta \delta]$ if one of the buses connected by the two lines on outage is the swing bus, then the dimension of the above matrix will be $3 \times 3$. The same procedure can be used for any outage level. 
The contingency analysis is faster with the use of $[B]^{-1}$. The advantage of using upper or lower triangular elements of $[\mathrm{B}]^{-1}$ lies in the smaller number of arithmetic operations required for complete contingency analysis. The main problem with $[\mathrm{B}]^{-1}$ is the large storage requirement to store the elements. Memory requirement is not a big issue with modern computers, but the computation time may, however, limit the size of the power system that can be analyzed. The above method is faster for higher level outages since recursive use is not needed for multiple outages by the above method.

\subsubsection{Linear Programming Model For Load Shedding And Generation Rescheduling}

In the event of constraint(s) violation, the system could be, according to the definitions, in the emergency or the extreme emergency state depending upon the corrective actions required to alleviate the constraint(s) violation and the amount of load curtailment required to alleviate the problem. The main objective, in this regard is, to alleviate that problem using rescheduling of generation without attempting load curtailment. If it is not possible to overcome the difficulty by rescheduling the generation then load will be curtailed from different buses. A linear programming method is used for generation rescheduling and load shedding. This linear programming model is given below:

Objective: Minimize Sum $=\sum_{i=1}^{\text {Nload }} \mathrm{X}_{\mathbf{i}}$

Such that

$$
\begin{aligned}
& \sum_{i=1}^{\text {Nload }} \mathrm{X}_{\mathrm{i}}+\sum_{i=1}^{N l o a d} \mathrm{P}_{\mathrm{Li}}+\sum_{i=1}^{N g b u s} \mathrm{P}_{\mathrm{Gi}}=0 \\
& \mathrm{~T}_{\mathrm{L}} \mathrm{I} \leq \mathrm{T}_{\mathrm{L}}{ }^{\mathrm{M}} \\
& \mathrm{P}_{\mathrm{Gi}}{ }^{\mathrm{m}} \leq \mathrm{P}_{\mathrm{Gi}} \leq \mathrm{P}_{\mathrm{Gi}}{ }^{\mathrm{M}} \\
& 0 \leq \mathrm{X}_{\mathrm{i}} \leq \mathrm{P}_{\mathrm{Li}}
\end{aligned}
$$

Where, Nload is the number of load points and Ngbus is the number of generation buses in the system. In this model, constraint 4.19 reflect active power balance, constraint 4.20 the so-called $\mathrm{N}$ and $\mathrm{N}-1$ security limits of the transmission line flow, constraint 4.21 limits of generation and constraint 4.22 limit the amount of total load curtailment. In this 
model both the generation shift and the load shed are used as variables. The main advantage of this model is that for each of those contingencies which violate the overload constraint, both the generation rescheduling as well as the amount of load shed can be found from a single run.

\subsection{LINEAR PROGRAMMING ALGORITHMS TO SOLVE THE MODEL}

Linear programming is one of the tools of operation research designed to assist in choosing among alternatives where limited resources preclude choosing all alternatives simultaneously. Linear programming deals with the problem of determining feasible plans which are optimal with respect to a linear objective function in particular, it determines a plan which maximizes or minimizes some linear function over all possible feasible plans. The feasible plans are those that satisfy certain restrictions which are usually in the form of a system of linear inequalities. There are a number of methods or algorithms for solving linear programs. The simplex method is a basic technique for solving optimization problems. The simplex method is based on solving a system of linear equations with the Gauss-Jordan [98] procedure. To apply the Gauss-Jordan procedure, the feasible region of the linear programming problem must be converted to a system of equalities by adding slack or artificial variables [98]. The simplex algorithm for solving linear programming is described below.

\section{Simplex Algorithm}

Consider the system of equations after conversion to canonical form is given by

$$
[\mathrm{A}][\mathrm{X}]=[\mathrm{b}]
$$

which maximizes

$$
z=[c][X]
$$

where:

$$
\begin{aligned}
& {[A]=\left(\frac{n x m}{1}\right) \text { coefficients matrix and } n>m} \\
& {[X]=(n x 1) \text { vector of variable } x_{i} \geq 0,}
\end{aligned}
$$


[c] $=(1 \mathrm{xn})$ penalty or cost vector for variables $\mathrm{x}_{\mathrm{i}}$ and

[b] $=$ resource vector and all $b_{i} \geq 0$.

Under the assumption that an initial basic feasible solution has been already found, the iterative steps of the simplex algorithm for the above generalized linear model can be stated as:

1. calculate $\mathrm{z}_{\mathrm{j}}-\mathrm{c}_{\mathrm{j}}=\sum_{i=1}^{m} c_{i} a_{i j}-c_{j}$ for each variable not in the present solution i.e. for each of the nonbasic variables.

a. if, for at least one $j, z_{j}-c_{j}$ is negative and at least one $a_{i j}$ for that $j$ is positive, a better feasible solution is possible. Let the column $\mathrm{k}$ has the most negative $z_{j}-c_{j}$ value.

$b$. if, for one $j, z_{j}-c_{j}$ is negative but all $a_{i j}$ for that $j$ are negative, the objective function is not bounded.

c. if $z_{j}-c_{j}$ is not negative for any $j$, the solution is optimum.

2. calculate $\theta$ using $\theta=\min \left[b_{i} / a_{i k}\right]$ for $a_{i k}>0$ and $i=1$ to $\mathrm{m}$. Let minimum value of $\theta$ occur at row $r$.

3. the element $\mathrm{a}_{\mathrm{rk}}$ is the pivotal element. Divide the $\mathrm{r}^{\text {th }}$ row through by $\mathrm{a}_{\mathrm{rk}}$ to reduce $\mathrm{a}_{\mathrm{rk}}=1$ and perform the row operations that will reduce all $\mathrm{a}_{\mathrm{ik}}$ to zero.

4. repeat step 1,2 and 3 until condition $1(c)$ is obtained.

The above algorithm for the maximization problem can be used to solve a minimization problem by multiplying the objective function by -1 and then multiplying the optimum value by -1 .

In the above simplex algorithm, the variable $x_{j}$ has upper and lower bound limits of infinity and zero respectively. In most power system analysis, all the variable has finite upper and lower bound limits. The above algorithm is not suitable for this type of application because of the amount of computation and the memory requirement. The bounded variable algorithm described below which is a modification of the above simplex algorithm is more efficient for this type of application. 


\subsubsection{Primal Simplex Algorithm With Bounded Variable}

In LP problems, constraints of the following form often occur

$$
\mathbf{l}_{\mathbf{j}} \lesseqgtr \mathbf{x}_{\mathbf{j}} \fallingdotseq \mathbf{u}_{\mathbf{j}}
$$

where $u_{j}$ and $l_{j}$ are the upper and lower bounds of the $x_{j}$ variable. In the simplex algorithm given above $l_{j}=0$ and $u_{j} \rightarrow$ inf. The constraints given by Equation 4.24 can be incorporated into the problem as ordinary constraints in order to use the simplex algorithm noted earlier. The number of constraints in the problem is now $m+2 n$ if every variable has finite upper and lower bounds and $\mathrm{m}$ is the number of constraints originally in $[A]$ and $n$ is the number of variables. The bounded variable algorithm described below keeps $m$ constraints and thus reduces the size of the tableau i.e. the memory requirements and the number of numerical computations.

\section{Upper bound}

Consider the following set of equations

$$
\begin{array}{ll}
z_{0}-c_{1} x_{1}-c_{2} x_{2} & =v \\
a_{11} x_{1}+a_{12} x_{2}+x_{3} & =b_{1} \\
a_{21} x_{1}+a_{22} x_{2}+x_{4} & =b_{2}
\end{array}
$$

where each variable $x_{j}$ has an upper bound $u_{j}$. Consider that in Equation $4.25-c_{1}$ is the most negative element in the objective function. Then according to the simplex algorithm, $x_{1}$ will be increase from 0 to $\min \left\{b_{i} / a_{i 1}\right.$ given $\left.a_{i 1}>0\right\}$. The minimum of $\left\{b_{i} / a_{i 1}\right.$ given $\left.a_{i 1}>0\right\}$ could be greater than $u_{1}$, the upper bound of $x_{1}$. Therefore the maximum value that $x_{1}$ can attain is

$$
\min \left[\min \left(b_{\mathrm{i}} / \mathrm{a}_{\mathrm{i} 1} \text { given } \mathrm{a}_{\mathrm{i} 1}>0\right), \mathrm{u}_{1}\right]
$$

If the minimum value is the $\min \left\{b_{i} / a_{i 1}\right.$ given $\left.a_{i 1}>0\right\}$ for some $i=r$, then the usual simplex method is used with $\mathrm{a}_{\mathrm{r} 1}$ as a pivot to transform Equation 4.25 to a new tableau. On the other hand, if the minimum value is $u_{1}$ then Equation 4.25 has to be modified using $x_{1}$ assumed to be its upper bound. This can be done by replacing $x_{1}=u_{1}-x^{*}{ }_{1}$ where $x^{*}{ }_{1}$ is a slack variable incorporated to guaranteed the inequality $x_{1} \leq u_{1}$. Thus when $\mathrm{x}^{*}{ }_{1}=0, \mathrm{x}_{1}=\mathrm{u}_{1}$ and when $\mathrm{x}^{*}{ }_{1}=\mathrm{u}_{1}$ then $\mathrm{x}_{1}=0$, substituting $\mathrm{x}_{1}=\mathrm{u}_{1}-\mathrm{x}^{*}{ }_{1}$ in Equation 4.25 gives 


$$
\begin{aligned}
& \mathrm{z}_{0}+\mathrm{c}_{1} \mathrm{x}^{*}{ }_{1}-\mathrm{c}_{2} \mathrm{x}_{2} \quad=\mathrm{v}+\mathrm{c}_{1} \mathrm{u}_{1} \\
& -a_{11} x^{*}{ }_{1}+a_{12} x_{2}+x_{3} \quad=b_{1}-a_{11} u_{1} \\
& -a_{21} x^{*}{ }_{1}+a_{22} x_{2}+\quad x_{4}=b_{2}-a_{21} u_{1}
\end{aligned}
$$

The tableau has been changed, therefore, as

1. the sign of all coefficients of $x_{1}$ is changed

2. the right hand side of all constraints is changed by $b_{i}=b_{i}-a_{i 1} u_{1}$ including the objective function which is changed to $v=v+c_{1} u_{1}$

In the above tableau $x^{*}{ }_{1}$ is nonbasic and hence $x^{*}{ }_{1}=0$ giving $x_{1}=u_{1}$. In a later iteration $\mathrm{x}^{*}{ }_{1}$ could become basic and may attain its upper bound $\mathrm{u}_{1}$. If this is the case, similar substitution as mentioned earlier or the above two rules can be followed with $x_{1}$ now a nonbasic variable. In the final solution, if $x_{j}$ is in the tableau, then $x_{j}$ is the the right hand side corresponding to the $x_{j}{ }_{j}$ basic row. If $x_{j}^{*}$ is in the tableau, then $x_{j}^{f_{j}}=u_{1}-x_{j}^{*}$.

In the above procedure, there is a possibility that due to the increase in $x_{1}$, the present basic variables may exceed their upper bounds. For example, with $x_{2}$ as nonbasic, from Equation 4.25,

$$
\begin{aligned}
& x_{3}=b_{1}-a_{11} x_{1} \\
& x_{4}=b_{2}-a_{21} x_{1}
\end{aligned}
$$

Hence the upperbounds of $u_{3}$ or $u_{4}$ can be reached if

$$
\begin{aligned}
& b_{1}-a_{11} x_{1}=u_{3} \text { or } \\
& b_{2}-a_{21} x_{1}=u_{4} .
\end{aligned}
$$

Therefore, $\mathrm{x}_{1}$ can not be increased above

$$
\min \left\{b_{i}-u_{i} / a_{i 1} \text { given } a_{i 1}<0\right\}
$$

where $u_{i}$ is the upper bound of the basic variable in row $i$. Therefore, the maximum value of $x_{1}$ that can attained is

$$
\min \left[\min \left\{b_{\mathrm{i}}-\mathrm{u}_{\mathrm{i}} / \mathrm{a}_{\mathrm{i} 1} \text { given } \mathrm{a}_{\mathrm{i} 1}<0\right\}, \min \left\{\mathrm{b}_{\mathrm{i}} / \mathrm{a}_{\mathrm{i} 1} \text { given } \mathrm{a}_{\mathrm{i} 1}>0\right\}, \mathrm{u}_{1}\right]
$$

Assuming that minimum occurs at $\mathrm{u}_{3}$, the upper bound of $x_{3}$, first $x_{3}$ is set to its upper bound by substituting in Equation $4.25 \mathrm{x}_{3}=\mathrm{u}_{3}-\mathrm{x}_{3}{ }_{3}$ and then the tableau is updated by using transformation with $\mathrm{a}_{11}$ as pivot. 
The rules for modifying the simplex algorithm are summarized as follows:

1. New basic variable: select the column $\mathrm{k}$, for the new basic variable as in the usual simplex method

2. Leaving basic variable: select the row $r$, with $\min \left\{\theta_{1}, \theta_{2}, \theta_{3}\right\}$ where

a. $\theta_{1}=\min \left\{b_{i} / a_{i k}\right.$ given $\left.a_{i k}>0\right\}$

b. $\theta_{2}=\min \left\{b_{\mathrm{i}}-\mathrm{u}_{\mathrm{i}} / \mathrm{a}_{\mathrm{ik}}\right.$ given $\left.\mathrm{a}_{\mathrm{ik}}<0\right\}$

c. $\theta_{3}=u_{k}$

for $i=1$ to $\mathrm{m}$. In the case of equal values, it is better to select $\theta_{3}$ since the computation is relatively easier.

3. Transformation of the tableau:

a. $\theta=\theta_{1}$, normal simplex method with $\mathrm{a}_{\mathrm{rk}}$ as pivot

b. $\theta=\theta_{2}$

i. change the upper bound switch on the vector that will leave the basis

ii. change the right hand side of the $r^{\text {th }}$ row to $b_{r}-u_{r}$

iii. multiply the basic vector which is to leave the basis by -1

iv. transform the tableau with $\mathrm{a}_{\mathrm{rk}}$ as pivot

c. $\theta=\theta_{3}$

i. change the upper bound switch on the $\mathrm{k}^{\text {th }}$ vector

ii. update the right hand side values $b_{i}-a_{i k} u_{k}(i=1$ to $m$ )

iii. multiply all coefficients of the $\mathrm{k}^{\text {th }}$ vector by -1

\section{Lower Bound}

The inclusion of the lower bound is relatively easier than the upper bound problem. Only a substitution is required for those variables which have lower bound constraints. Consider the constraints of the form

$\mathrm{x}_{\mathrm{i}} \geq \mathrm{l}_{\mathrm{i}}$ 
The above constraint can be written as

$$
\begin{aligned}
x_{i}-l_{i} & \geq 0 \\
\text { or, } x^{*} & \geq 0
\end{aligned}
$$

where $\mathrm{x}^{*}{ }_{\mathrm{i}}=\mathrm{x}_{\mathrm{i}} \mathrm{l}_{\mathrm{i}}$ and $\mathrm{x}_{\mathrm{i}}^{*} \geq 0$. Therefore, the usual method as explained earlier can be used with the substitution of the variables $x_{i}$ by $x_{i}^{*}$. The solution of the LP model will satisfy the constraint 4.27 given above because

$$
\text { when } \begin{aligned}
\mathrm{x}_{\mathrm{i}}^{*} \geq 0 \text { then } \mathrm{x}_{\mathrm{i}} & =1_{\mathrm{i}}+\mathrm{x}^{*} \mathrm{i} \\
& \geq 0 .
\end{aligned}
$$

\subsubsection{Dual Simplex Method For Bounded Variables}

The dual simplex method is very efficient when adding constraints to a LP model in which an optimal solution has already been determined or when a new resource vector $b^{*}$ is introduced. In the original simplex algorithm the first tableau starts with a basic feasible solution, but where all $z_{j}-c_{j}$ are not necessarily nonnegative. Necessary changes in the basis are made, one at a time, maintaining nonnegativity of the variables until all $\mathrm{z}_{\mathrm{j}}-\mathrm{c}_{\mathrm{j}}$ components are nonnegative at which point the solution is the optimal feasible solution. The dual simplex method starts with a basic solution that is not feasible but where all $z_{j}-c_{j}$ components are nonnegative. In the primal simplex method, the variable with most negative $z_{j}-c_{j}$ enters the solution and the variable leaves the solution according to the $\theta$ rule which will retain feasibility of the solution. In the dual simplex method, the variable which leaves the solution is selected first according to which variable is most infeasible and then the variable which will enter the solution is selected in such a way that the optimality condition i.e. all $z_{j}-c_{j}$ nonnegativity is retained until a feasible solution is reached at which point the solution is optimal. The dual simplex algorithm is as follows:

1. for those variables which exceeded the upper bound, use the substitution as explained before.

2. If there are negative basic variables in the present solution, select the most negative one to leave the basic solution to determine the pivot row i.e. select the row $\mathrm{r}$ such that

$$
b_{r}=\min \left[\left(b_{i} / a_{i i}\right] \text { for } i=1 \text { to } m \text { and } b_{i} \text { and } a_{i i}\right. \text { have opposite sign. }
$$


3. Select the nonbasic variable to enter the basic solution by taking the cost row coefficient to the pivot row coefficient in each nonbasic vector while ignoring positive and zero denominators. Select the vector associated with the largest algebric ratio i.e. select column $\mathrm{k}$ such that for $j=1$ to $n$.

$$
\theta_{\mathrm{k}}=\max \left[\mathrm{z}_{\mathrm{j}}-\mathrm{c}_{\mathrm{j}} / \mathrm{a}_{\mathrm{rj}}\right] \text { given } \mathrm{a}_{\mathrm{rj}}<0
$$

4. $\mathrm{a}_{\mathrm{rk}}$ is the pivotal element. Perform a normal row operation of the simplex method.

5. Repeat step 1, 2 and 3 until the solution is optimal.

The simplex method of linear programming is described in some detail as it is an integral part of the security evaluation research performed in this project. The dual simplex method is used in the load curtailment and generation rescheduling analysis in this chapter. The primal simplex method is used in the voltage correction analysis conducted in Chapter 5.

\subsection{MODIFIED RBTS (MRBTS)}

The 6-bus RBTS [91] has been modified to a 5 bus system having 8 lines by connecting at bus 5 the load of bus 6 plus the base case power flow loss of line 9 as shown in Figure 4.3. This modification was done to remove the radial line between buses 5 and 6 , as the probability of the normal state will be always zero because the outage of line 9 (single level contingency) will isolate bus 6 and result in the curtailment of $20 \mathrm{MW}$ of load at bus 6 .

\subsection{SPLIT NETWORK SITUATION}

Changes in the network configuration due to the outages of line(s) and/or transformer(s) may result in the splitting of a network into two or more than two smaller networks. Each network may consists of PQ buses and/or PV buses. Under steady state conditions, they can be treated as separate independent networks and reliability indices can be computed by considering each network separately. The most appropriate technique for this purpose is to recompute the system matrices [B'] and [B"] for each of the subnetworks and then use an ac load flow to determine the system problem(s). This technique requires large computation time to recompute and factorizes the system 


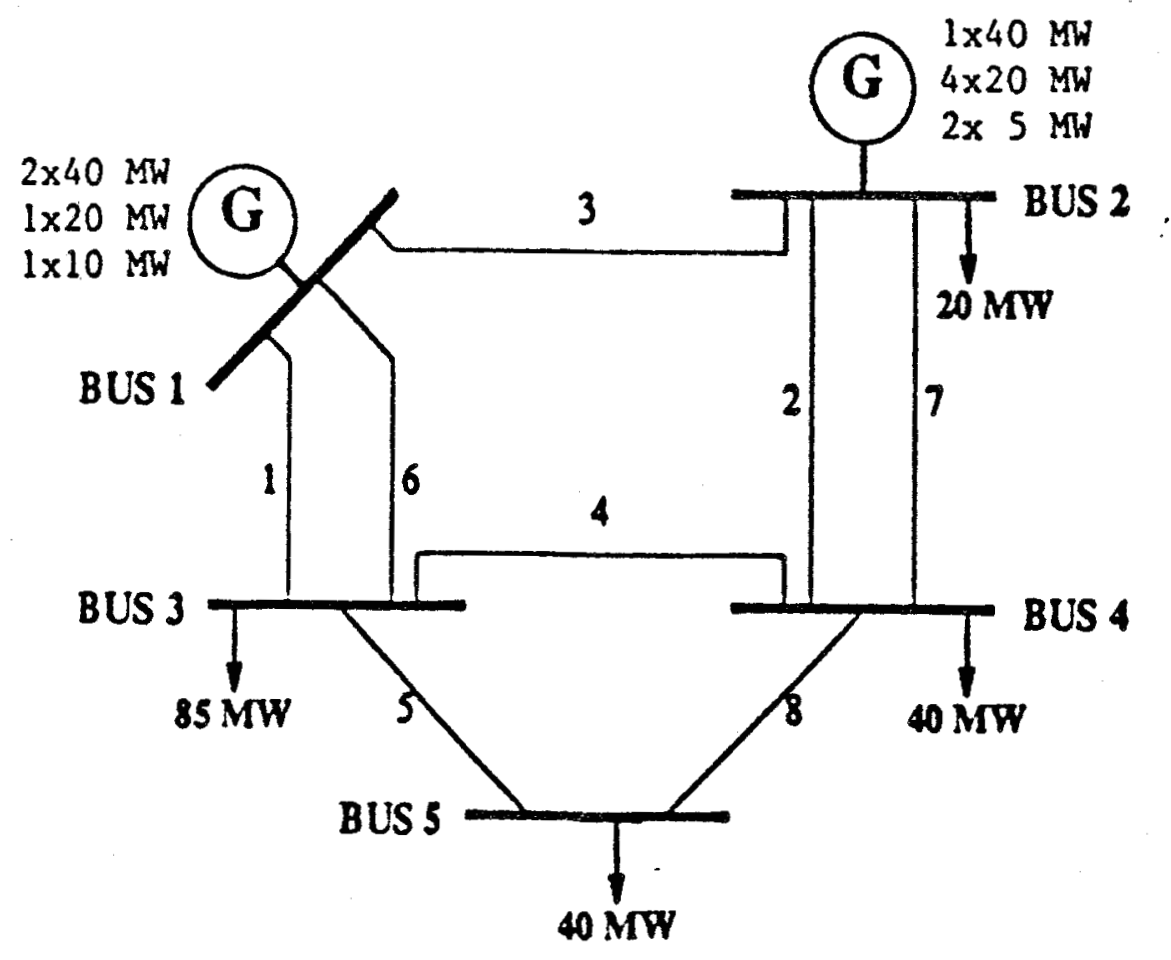

Figure 4.3: Modified RBTS

matrices for each of the networks. This method also needs additional memory to store the above matrices for each of the networks.

The split network situation usually occurs by an outage event involving at least two or more than two transmission lines in a practical system. The probabilities and frequencies of these independent outages are very small and therefore their contribution to the indices are not significant. Table 4.1 shows the total number of split networks caused by transmission lines and/or transformer outages for different levels of line 
contingencies. It can be seen from Table 4.1 that no first level outages cause split networks for both the test systems, only one second level outage creates a split network for the IEEE-RTS and the remainder are due to third level outages. The probabilities and

Table 4.1: Number of split network caused by transmission lines outages

Contingency

Level Number Of Split Networks MRBTS RBTS IEEE-RTS

From First

From Second

From Third
0

0

2

0
1
10

0

1

50

frequencies of these events as noted earlier are very small and hence an approximate method can be used to reduce the computation time. The following approximate method has been used in this research work to solve split network situations.

\subsubsection{Approximate Method For Split Network Situation Solution}

Consider the modified RBTS (MRBTS) configuration. In this case, the system is split into two networks for the outage of lines 3,4 and 5 or for the outage of lines $3,4,8$, for example. One subnetwork consists of buses 1 and 3 and the other subnetwork consists of buses 2,4 and 5 for the outage of lines 3,4 and 5 . In this case both the networks have both $\mathrm{PV}$ and $\mathrm{PQ}$ buses. The resulting configuration can be shown as Figure 4.4 where the split network situation occurs with the outage of three lines. Lines on outage can be ideally represented as lines in service having infinite impedances. It

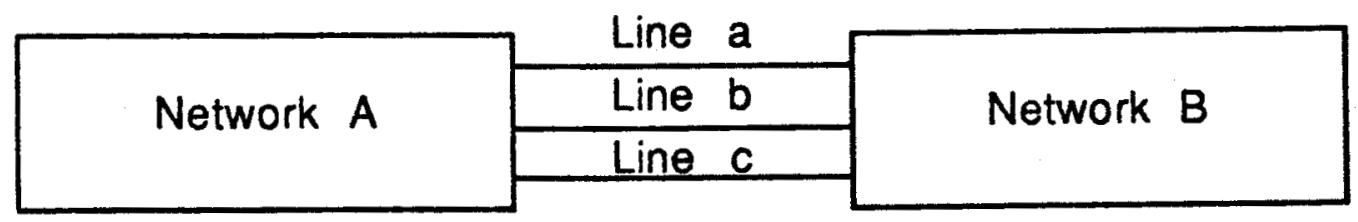

Figure 4.4: Split network situation 
can, therefore, be assumed that networks A and B in Figure 4.4 are connected by 3 lines having very high impedances. The power flow through these lines will be very small due to the high impedances and as essentially these lines are not connecting the networks together. The change to high impedance values can be easily incorporated using Woodbury's formula [99]. In order to further limit the power flows, the capacity of these lines can be assumed to be close to zero in the LP model. Considering the power flows through these lines to be equal to zero dictates that no power will flow through these lines in the LP model and it can be assumed that there is no connection between the networks since no power export/import occurs between them. Considering only the condition of zero power flow in the LP model may give satisfactory results in some cases. In most cases, however, the results will be unsatisfactory because the angle difference between the two ends of the lines must be equal to zero in order to keep the power flows zero. To keep the angles equal between the two buses connected by the lines may cause more load curtailment. The total load curtailments in the two test systems using only the zero power limit condition are shown in Table 4.2 for a few split situations. It can be seen from this table that for the outage of lines 3,4 and 5 in the MRBTS, the total load curtailment is $54.6 \mathrm{MW}$. The zero power limit of line 3 causes $\delta_{1}=\delta_{2}$ and similarly zero power limits of lines 4 and 5 cause $\delta_{3}=\delta_{4}=\delta_{5}$. Therefore, bus 5 experiences a total load curtailment since no power can flow through line 8. Some load is also curtailed from bus 4 due to the above angle restriction giving a total of $54.6 \mathrm{MW}$. It should be noted that with the above lines on outage, both the subnetworks have sufficient reserve and line capacity to supply the total system load. The load curtailments for the IEEE-RTS are excessive in all most all cases as can be found by comparing the results of Table 4.2 with Table 4.3. Table 4.3 shows the load curtailment considering both the conditions noted earlier. Therefore, both the conditions mentioned earlier i.e. high impedance and low power flow capacity have been used for those lines which cause a split network situation during the solution of this outage. The Woodbury formula has been used to update the $\left[\mathrm{B}^{\prime}\right]^{-1}$ matrix using high impedances for the lines which cause a split network. The LP model is developed with the updated matrix and using normal line power capacities for the lines not on outage. The lines on outage which cause a split network are considered to be in service with low power flow capacities and high impedances. Some of the load curtailment results are shown in Table 4.3 for the two test systems. 
Table 4.2: Load curtailment considering zero line capacity only in the LP model

$\begin{array}{lcc}\text { Test } & \text { Split Network } & \text { Load Curtailment } \\ \text { System } & \text { Due To Outage Of } & \text { (MW) }\end{array}$

$\underline{\text { MRBTS }}$

Lines $3,4,5$

54.6

Lines $3,4,8$

$\underline{\text { IEEE-RTS }}$

$\begin{array}{lr}\text { Lines } 12,13 & 60.3 \\ \text { Lines } 7,19,29 & 475.0 \\ \text { Lines } 29,36,37 & 309.0\end{array}$

Table 4.3: LP solution for the split network situations

\begin{tabular}{|c|c|c|c|}
\hline $\begin{array}{l}\text { Test } \\
\text { System }\end{array}$ & $\begin{array}{c}\text { Split Network } \\
\text { Due To Outage Of }\end{array}$ & $\begin{array}{l}\text { Load Curtailment } \\
\text { (MW) }\end{array}$ & $\begin{array}{l}\text { NF Method } \\
\text { (MW) }\end{array}$ \\
\hline
\end{tabular}

MRBTS

$\begin{array}{lrr}\text { Lines } 3,4,5 & 0.0 & 0.0 \\ \text { Lines } 3,4,8 & 15.1 & 15.1\end{array}$

$\underline{\text { IEEE-RTS }}$

$\begin{array}{lrr}\text { Lines } 12,13 & 0.0 & 0.0 \\ \text { Lines } 1,4,10 & 41.0 & 41.0 \\ \text { Lines } 1,8,10 & 115.0 & 115.0 \\ \text { Lines } 7,19,29 & 0.0 & 0.0 \\ \text { Lines } 7,23,29 & 165.0 & 165.0 \\ \text { Lines } 25,26,28 & 212.0 & 212.0 \\ \text { Lines } 29,36,37 & 309.0 & 309.0\end{array}$




\section{$\underline{\text { MRBTS }}$}

Two third level contingencies result in a split network situation for this system. The split network caused by the outage of lines 3, 4 and 5 requires no load curtailment since both the subnetworks have sufficient generation available. The outage of lines 3,4 and 8 , however, requires load curtailment due to the shortage of power in the subnetwork consisting of buses 1,3 and 5 .

\section{IEEE-RTS}

The outage of lines 12 and 13 results in a split network situation. Buses 7 and 8 form a small subnetwork and the remaining buses form a big subnetwork. There is no load curtailment for this outage since both the subnetworks have sufficient generation to supply the load within the line flow constraints. The outage of lines 29,36 and 37 cause two load buses 19 and 20 to form a subnetwork. Since neither of these buses has local generation, each bus experiences a total load curtailment. The other buses in the second subnetwork do not experience any load curtailment because of the $864 \mathrm{MW}$ reserve.

\subsection{FLOW CHART FOR DETECTING THE STATES}

A flow chart for detecting the different operating states is shown in Figure 4.5 for up to second level outages. This concept can be extended to include higher order outages in different states. The "problem" block depends on the purpose of the study as noted earlier. The "decision" block depends on the planners point of view. If the amount of MW curtailment is an important consideration, then all the combinations with the present outage must be solved to determine the actual MW curtailment. On the otherhand if only probability and frequency are required, then the contribution of the higher level outages can be included without solving them explicitly. 


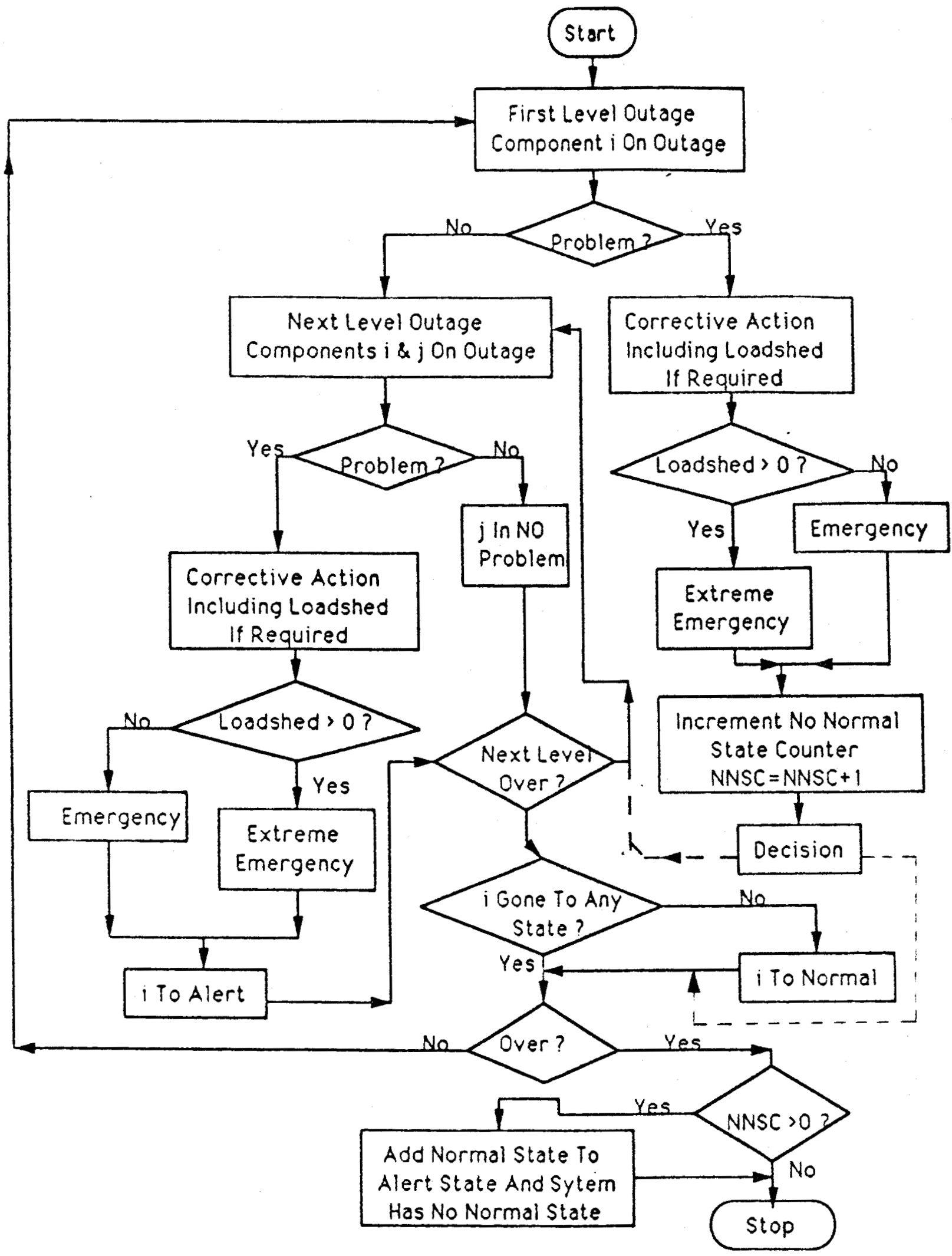

Figure 4.5: Flow chart for detecting different operating states 


\subsection{STUDY RESULTS}

The probabilistic indices for different operating states have been calculated using the two test systems described earlier. The results presented in this chapter consider up to the fourth level of generator outages, third level of line outages and third level of generator and line combination outages. In these studies, $99.998 \%$ of the total probability for the MRBTS and $98.643 \%$ of the total probability for the IEEE-RTS can be captured by considering these outages. The remaining probabilities are contributed by higher level contingencies which are not considered. The results shown here are for the constraint set 1 defined earlier.

\subsubsection{MRBTS Results}

The probabilities and frequencies of different system operating states are shown in Table 4.4. These indices are annualized indices for the this test system as the load is

Table 4.4: Probability and frequency of different operating states - MRBTS

\begin{tabular}{lrr}
\hline System & Probability & Frequency \\
State & & \\
\hline Normal & 0.813090 & 51.976677 \\
Alert & 0.177980 & 56.966892 \\
Emergency & 0.000137 & 0.207265 \\
Ext. Emergency & 0.008589 & 3.988032 \\
No problem & 0.000179 & 0.238960 \\
\hline
\end{tabular}

Total considered

0.999975

considered to be constant at $185 \mathrm{MW}$ throughout the year. It can be seen from this table that the probability of the normal state is greater than zero which means that there are no single level contingencies which create line overload problems for this modified test system. The probability and frequency of the extreme emergency state is higher than the probability and frequency of the emergency state. This is because of the fact that most of 
the problem creating contingencies, especially the higher level generator outages, require load curtailment at this load level to overcome the problem. This may not be true for other load levels. The 'probability of no problem' is the sum of the probabilities of those third or fourth level contingencies which do not create system problems and since a decision can not be taken for these contingencies to which state they belong, they are added to represent the probability of no problem creating contingencies. The probability and frequency of no problem, according to the definition of the system operating states, belongs to either the normal state or the alert state depending upon whether the next associated higher level contingencies create problems or not. The probability of the extreme emergency state is the probability of load curtailment. Therefore, for this test system, the probability of load curtailment is $0.8589 \%$ of the total probability considered. The system operates without violating limits in both the normal and the alert states and therefore, the sum of these probabilities represents the reliability of the system. The complement of this value can be considered as a "Risk" index which is defined in this studies as

$$
\text { Risk }=1.0-P_{n}-P_{a}-P_{n p}
$$

where $P_{n}$ is the probability of the normal state, $P_{a}$ is the probability of the alert state and $P_{n p}$ is the probability of the no problem situations. This risk index can be used in system expansion planning and this application is demonstrated in Chapter 7. The risk index for this system is 0.008751 .

\subsubsection{IEEE-RTS Results}

In order to avoid the situation in which the probability of the normal state is zero, the first level outage of line 11 and associated higher level outages with this line (from line 12 to 38 and generators) for the IEEE-RTS are ignored, as due to this outage one of the generator buses becomes isolated. The isolation of a bus is considered as a system problem and therefore this isolation for a first level outage is ignored in order not to violate the the definition of the normal state. This isolation of a bus will cause the probability of the normal state to be zero even if there is no load curtailment. The annualized indices for this test system are shown in Table 4.5 for the condition in which the load throught out the year is equal to the peak load of $2850 \mathrm{MW}$. The probability of 
Table 4.5: Probability and frequency of different operating states - IEEE-RTS

\begin{tabular}{lrr}
\hline $\begin{array}{llr}\text { System } \\
\text { State }\end{array}$ & Probability & Frequency \\
& & \\
\hline Normal & 0.551151 & 231.093837 \\
Alert & 0.327084 & 166.586846 \\
Emergency & 0.000001 & 0.001412 \\
Ext. Emergency & 0.086144 & 53.915508 \\
No problem & 0.021227 & 21.202090 \\
\hline & & \\
\hline & & \\
\hline
\end{tabular}

the normal state is greater than zero for this system as no single level contingencies create overload problems. The probability and frequency of the extreme emergency state is higher than the probability and frequency of the emergency state. The reason is similar to that described for the MRBTS. The probability of load curtailment is 0.086144 which is 8.74 percent of the total probability considered in the studies. The risk index for this system is 0.100538 .

\subsection{EFFECT OF LOAD VARIATION AND ANNUAL INDICES}

In a practical system, the load does not stay at its peak value throughout the year. An evaluation of the system performance assuming a peak load model may therefore give highly pessimistic values for the reliability indices. These indices, referred to as annualized indices, are useful for comparing the performance of two or more systems but do not convey accurate information about the overall quantitative evaluation of a power system. Modelling the system load as a multistep load provides more accurate results than the single step load model. In order to calculate annual indices, 8736 system hourly loads must be considered in the study. This representation, depending on the size of the system, will require considerable computation time and thus the load models have to be reduced to a smaller number of states. 
A proper selection of the number of load states is primarily dictated by the shape of the load curve, the size of each step, the contribution of the lowest step load to the indices and the period for which the lowest step load exists. This is due to the fact that even if the contribution of the lowest step load is quite low as compared to the highest step load but depending upon the duration for which each load step exists, the contribution of each load step to the annual indices may be comparable. In order to show the effect of a multistep load model and to calculate the annual indices, a 'seven-step' load model for the system peak load curve as shown in Figure 4.6 has been considered. The load steps

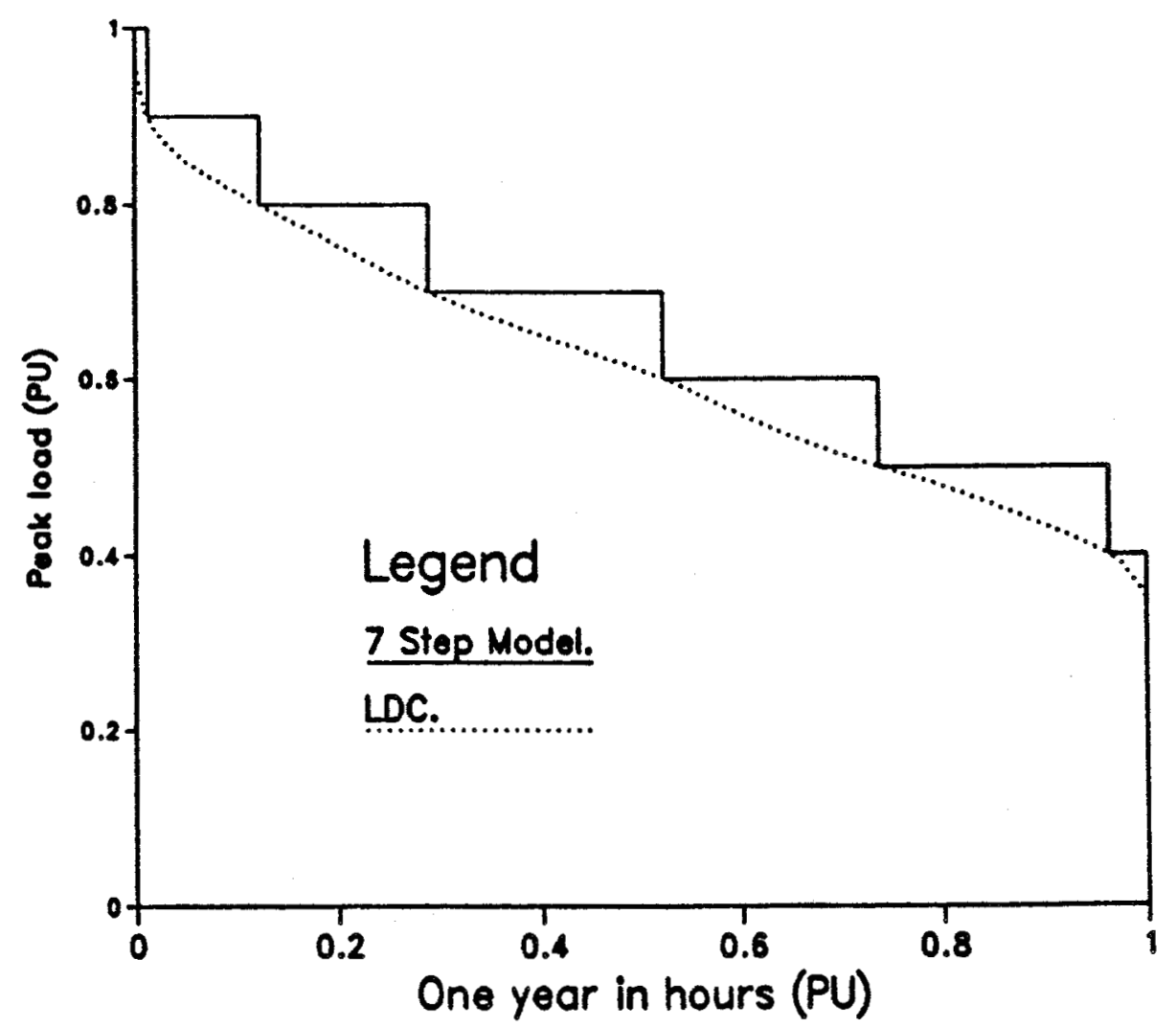

Figure 4.6: Multi-step load model

and the probabilities of each load step are shown in Table 4.6. The effect of varying load on the indices of different operating states is discussed in this section. An attempt has been made to determine the proper number of load steps required to calculate the indices for both systems.

In assigning values of the active and reactive load at each bus for each load step, it has been assumed that the load at each bus varies in proportion to the system load. If the 
Table 4.6: Probability of different load level

\begin{tabular}{ccc}
\hline Load Step & \% Load & Probability \\
\hline 1 & 40 & 0.03651557 \\
2 & 50 & 0.22630495 \\
3 & 60 & 0.21554486 \\
4 & 70 & 0.23202839 \\
5 & 80 & 0.16540751 \\
6 & 90 & 0.11103480 \\
7 & 100 & 0.01316392 \\
\hline
\end{tabular}

system peak load is $X$ and the corresponding peak loads at buses $i$ and $j$ are $X_{i}$ and $X_{j}$ then for a step system load of $Y$, the corresponding real step loads $Y_{i}$ and $Y_{j}$ at buses $i$ and $\mathrm{j}$ are:

$$
\begin{aligned}
& Y_{i}=X_{i}(Y / X) \text { and } \\
& Y_{j}=X_{j}(Y / X) .
\end{aligned}
$$

The reactive loads at buses $i$ and $j$ are:

$$
\begin{aligned}
& R_{i}=Y_{i} \tan \tau_{i} \\
& R_{j}=Y_{j} \tan \tau_{j}
\end{aligned}
$$

where $\tau_{i}$ and $\tau_{j}$ are load power factors angle at buses $i$ and $j$ respectively.

Numerical values of the active load at each bus are shown in Table 4.7 for the MRBTS. In practical situations, complete load correlation may not exist and there could be many ways in which the system load is shared between the system buses. In such cases, if the load pattern at each individual bus is known, indices can be evaluated for the specific values of real and reactive loads.

The effect of the load variations on different indices are shown in Table 4.8 through Table 4.11. It can be seen from these tables that the probability and the frequency for the 
Table 4.7: Bus loads in MW for the seven step load model - MRBTS

\begin{tabular}{|c|c|c|c|c|c|c|c|}
\hline \multirow[t]{2}{*}{ Bus } & \multirow[b]{2}{*}{$1 s t$} & \multirow[b]{2}{*}{$2 \mathrm{nd}$} & \multicolumn{2}{|c|}{ Step Number } & \multirow[b]{2}{*}{5 th } & \multirow[b]{2}{*}{ 6th } & \multirow[b]{2}{*}{ 7th } \\
\hline & & & 3rd & 4 th & & & \\
\hline 2 & 8.0 & 10.0 & 12.0 & 14.0 & 16.0 & 18.0 & 20.0 \\
\hline 3 & 34.0 & 42.5 & 51.0 & 59.5 & 68.0 & 76.5 & 85.0 \\
\hline 4 & 16.0 & 20.0 & 24.0 & 28.0 & 32.0 & 36.0 & 40.0 \\
\hline 5 & 16.0 & 20.1 & 24.1 & 28.1 & 32.1 & 36.1 & 40.1 \\
\hline Total & 74.0 & 92.6 & 111.1 & 129.6 & 148.1 & 166.6 & 185.1 \\
\hline
\end{tabular}

Table 4.8: Effect of load variation on the probability of different states - MRBTS

\begin{tabular}{rccccc}
\hline $\begin{array}{l}\text { Load } \\
\text { Step }\end{array}$ & Normal & Alert & $\begin{array}{c}\text { Probability of } \\
\text { Emergency }\end{array}$ & $\begin{array}{c}\text { Ext. } \\
\text { Emergency }\end{array}$ & $\begin{array}{c}\text { No } \\
\text { Problem }\end{array}$ \\
\hline & & & & & \\
\hline $40 \%$ & 0.982089 & 0.017379 & 0.000097 & 0.000001 & 0.000408 \\
$50 \%$ & 0.982142 & 0.017331 & 0.000091 & 0.000001 & 0.000409 \\
$60 \%$ & 0.982061 & 0.017412 & 0.000091 & 0.000003 & 0.000408 \\
$70 \%$ & 0.981151 & 0.018306 & 0.000091 & 0.000024 & 0.000402 \\
$80 \%$ & 0.977488 & 0.021828 & 0.000081 & 0.000186 & 0.000393 \\
$90 \%$ & 0.903998 & 0.093238 & 0.000076 & 0.002339 & 0.000324 \\
$100 \%$ & 0.813090 & 0.177980 & 0.000137 & 0.008589 & 0.000179 \\
annual & 0.970221 & 0.028863 & 0.000089 & 0.000410 & 0.000392 \\
\hline
\end{tabular}

Total probability considered in all cases $=0.999975$.

emergency state is higher than the corresponding values of the extreme emergency state for lower load levels. This is because the number of contingencies that require load curtailment at lower load levels is less than that at the system peak load. As can be seen 
Table 4.9: Effect of load variation on the frequency of different states - MRBTS

\begin{tabular}{rccccc}
\hline $\begin{array}{l}\text { Load } \\
\text { Step }\end{array}$ & $\begin{array}{c}\text { Normal } \\
\end{array}$ & Alert & $\begin{array}{c}\text { Frequency of } \\
\text { Emergency }\end{array}$ & $\begin{array}{c}\text { Ext. } \\
\text { Emergency }\end{array}$ & $\begin{array}{c}\text { No } \\
\text { Problem }\end{array}$ \\
\hline & & & & & \\
\hline $50 \%$ & 96.133540 & 16.542751 & 0.178764 & 0.002703 & 0.520069 \\
$50 \%$ & 96.188902 & 16.496736 & 0.166909 & 0.002774 & 0.522506 \\
$70 \%$ & 96.140157 & 16.545481 & 0.166672 & 0.004249 & 0.521268 \\
$80 \%$ & 95.731747 & 16.944926 & 0.166280 & 0.017278 & 0.517596 \\
$90 \%$ & 75.620309 & 17.986042 & 0.148587 & 0.112103 & 0.510786 \\
$100 \%$ & 51.976677 & 56.966892 & 0.139045 & 1.139277 & 0.424540 \\
annual & 92.941094 & 19.567528 & 0.161552 & 0.203192 & 0.504462 \\
\hline
\end{tabular}

from these tables, the annual indices for the normal state are much higher than the corresponding indices of the annualized peak load case. The extreme emergency state probability in both test systems is much lower than the corresponding annualized values. The annual indices provides a more realistic evaluation of the system than the annualized peak load indices.

\subsection{CONTINGENCY SORTING}

In a practical power system, there are many generators or lines which are identical in terms of their contribution to the system. For example, two or more identical lines may be connected between the same buses, i.e. parallel lines. The computation time for state evaluation can be reduced greatly by sorting out the identical contingencies. This effect is shown in Table 4.12. The system states considered without sorting for the MRBTS are 1489. The number of states considered up to the same contingency level with sorting of generating units only are 566. The number of contingency cases that have to be examined with sorting of both generators and lines remains the same because of the numbering of the lines in the MRBTS. The total system states considered without sorting 
Table 4.10: Effect of load variation on the probability of different states - IEEERTS

\begin{tabular}{rlllll}
\hline $\begin{array}{l}\text { Load } \\
\text { Step }\end{array}$ & Normal & Alert & $\begin{array}{c}\text { Probability of } \\
\text { Emergency }\end{array}$ & $\begin{array}{c}\text { Ext. } \\
\text { Emergency }\end{array}$ & $\begin{array}{c}\text { No } \\
\text { Problem }\end{array}$ \\
\hline $40 \%$ & 0.939258 & 0.005385 & 0.000000 & 0.000003 & 0.040960 \\
$50 \%$ & 0.939258 & 0.005385 & 0.000000 & 0.000003 & 0.040960 \\
$60 \%$ & 0.934976 & 0.009667 & 0.000004 & 0.000003 & 0.040957 \\
$70 \%$ & 0.934976 & 0.009667 & 0.000004 & 0.000003 & 0.040957 \\
$80 \%$ & 0.927453 & 0.016818 & 0.000004 & 0.000993 & 0.040339 \\
$90 \%$ & 0.870083 & 0.069197 & 0.000004 & 0.011278 & 0.035045 \\
$100 \%$ & 0.551151 & 0.327084 & 0.000001 & 0.086144 & 0.021227 \\
annual & 0.922599 & 0.020513 & 0.000003 & 0.002553 & 0.039939 \\
\hline
\end{tabular}

Total probability considered in all cases $=0.985606$

The outage for line 11 is neglected.

for the IEEE-RTS are 91560 . The number of states up to the same contingency level with sorting of generating units only are 24604. The number of contingency cases that have to be examined for the IEEE-RTS with generator and line contingency sorting is 19967. The comparison between the CPU times for sorting and without sorting can also be seen from Table 4.12. In the case of the IEEE-RTS, total saving in CPU time is quite significant. The CPU time with line and generator contingency sorting is less than $50 \%$ of that without sorting.

\subsection{SUMMARY}

In this chapter, a composite generation and transmission system has been classified by different operating states which provide a framework for incorporating security considerations in system reliability assessment. A flow chart for detecting these operating states is also presented. Annualized indices obtained by considering the system load to be constant throughout the year and the annual indices by considering a seven- 
Table 4.11: Effect of load variation on the frequency of different states - IEEERTS

\begin{tabular}{rrrrrr}
\hline $\begin{array}{l}\text { Load } \\
\text { Step }\end{array}$ & $\begin{array}{c}\text { Normal } \\
\end{array}$ & Alert & $\begin{array}{r}\text { Frequency of } \\
\text { Emergency }\end{array}$ & $\begin{array}{c}\text { Ext. } \\
\text { Emergency }\end{array}$ & $\begin{array}{c}\text { No } \\
\text { Problem }\end{array}$ \\
\hline $40 \%$ & 429.936623 & 5.046882 & 0.000310 & 0.004611 & 37.811265 \\
$50 \%$ & 429.936623 & 5.046882 & 0.000310 & 0.004611 & 37.811265 \\
$60 \%$ & 428.392369 & 6.591136 & 0.004539 & 0.004611 & 37.807037 \\
$70 \%$ & 428.392212 & 6.591293 & 0.004523 & 0.004627 & 37.807037 \\
$80 \%$ & 424.413609 & 10.405730 & 0.004714 & 0.574050 & 37.401589 \\
$90 \%$ & 390.523121 & 41.435443 & 0.004908 & 7.492615 & 33.343604 \\
$100 \%$ & 231.093837 & 166.586846 & 0.001412 & 53.915508 & 21.202090 \\
annual & 421.338051 & 12.791375 & 0.003453 & 1.639912 & 37.026901 \\
& & & & & \\
\hline
\end{tabular}

The outage for line 11 is neglected.

Table 4.12: Comparison of contingency considered and computation time with and without sorting

Sorting

Sorteng

For MRBTS

No

Generator

Generator + Line

Contingency

CPU Time

For IEEE-RTS

No

Generator

Generator + Line
1489

566

566
1.82 Seconds

1.04 Seconds

1.04 Seconds

.


step load model are presented. A basic electric power utility objective should be to operate in the normal state with a high probability. A system may be capable of operating within constraints but may not be able to withstand outages, even single level. A LP model for generation rescheduling and load curtailment as well as a solution technique for split network situations are also presented.

The computation time can be reduced significantly by sorting identical contingencies. The effect of generation sorting and both line and generator sorting are illustrated in this chapter. The saving in computation time using sorting can be quite significant and as illustrated is more than 50\% for the IEEE-RTS.

The Sherman-Morrison correction formula is generally used to obtain power system network solutions under line outage conditions. A simple correction formula is used to adjust the base solution to effectively represent the line outages instead of rebuilding and refactorizing the system admittance matrices for each of the line contingencies. Multiple line outages are represented by applying the formula recursively, and updating the solution vector at each step. An efficient method which avoids the recursive use of the Sherman-Morrison correction formula for multiple line outages is also discussed in this chapter. This method, however, requires the storage of the upper or lower triangular elements of the inverse system susceptance matrices. Memory requirements which earlier played an important role in the practical application of adequacy analysis techniques, are no longer a major issue. Practical limits on computation time may, however, limit the size of the power system that can be analyzed. The speed of the analysis increases using $[\mathrm{B}]^{-1}$.

The framework proposed in Reference [69] has been extended. A procedure has been developed to quantify this framework in terms of the probabilities and frequencies of finding the system in the defined states. The security constraint set 1 used in the studies reported in this chapter does not include voltage problems. Proper voltage magnitude is an important factor in reliability studies. The operating states are examined by considering constraint set 2 and by considering the total constraint set in the next chapter. 


\section{VOLTAGE PROBLEM CONSIDERATIONS IN THE RELIABILITY ASSESSMENT OF COMPOSITE POWER SYSTEMS}

\subsection{INTRODUCTION}

The indices for different operating states have been calculated considering the overload problem in the previous chapter. The changes in the voltage magnitudes from the base case values were neglected. The occurrence of certain contingencies, mainly transmission line outages, may result in deviation of voltage magnitudes from acceptable limits at some of the load buses in the system. Good quality of service at a load point does not involve deviations in the voltage level at that bus beyond permissible limits. The acceptable voltage level at a bus, therefore, is an important factor in a reliability analysis. The effects on different operating states of considering bus voltage magnitudes outside the acceptable limits to be a system problem are presented in this chapter.

Many power utilities use dc load flow for reliability studies because they do not view voltage deviation as a failure but only as a minor problem which is normally rectified by transformer tap-settings, phase shifter adjustments and/or local reactive power generation. This gives an optimistic assessment of the system and does not permit a quantitative evaluation of the outage contingencies using voltage as a reliability criterion. It is considered desirable to use voltage as a reliability criterion and suitable corrective action(s) should be taken when a voltage problem is encountered. A LP model for voltage correction by changing the appropriate generation bus voltages and Q-load curtailment at different load buses (if required) has been developed and is presented in the following sections. 


\subsection{CONSIDERATION OF VOLTAGE PROBLEMS}

The decoupled load flow equation for the reactive load at load buses can be written as [100]

$$
[\mathrm{B}][\mathrm{V}]+\left[\mathrm{B}^{0}\right]\left[\mathrm{V}^{0}\right]=[\mathrm{Q} / \mathrm{V}]
$$

where $\left[\mathrm{V}^{0}\right]$ and $[\mathrm{V}]$ are the generator bus and load bus voltage magnitude vectors respectively. Assume that due to a contingency, the change in the load bus voltage vector required to bring the voltage violating buses within the voltage limits is $[\Delta \mathrm{V}]$ and the corresponding change required in the generator bus voltage vector is $\left[\Delta \mathrm{V}^{0}\right]$. Substituting these values in Equation 5.1 gives

$$
[\mathrm{B}][\mathrm{V}+\Delta \mathrm{V}]+\left[\mathrm{B}^{0}\right]\left[\mathrm{V}^{0}+\Delta \mathrm{V}^{0}\right]=[\mathrm{Q} / \mathrm{V}]
$$

After simplification and with the assumption that $[Q / V] \approx[Q][101,102]$, the above equation can be written as

$$
[\mathrm{B}][\Delta \mathrm{V}]+\left[\mathrm{B}^{0}\right]\left[\Delta \mathrm{V}^{0}\right]=0
$$

or

$$
\left[\mathrm{B}^{0}\right]\left[\Delta \mathrm{V}^{0}\right]=-[\mathrm{B}][\Delta \mathrm{V}]
$$

where:

[B] is a matrix of dimension $\left(\mathrm{N}_{\mathrm{q}} \mathrm{xN}_{\mathrm{g}}\right)$

[B] is a matrix of dimension $\left(\mathrm{N}_{\mathrm{q}}^{\mathrm{x}} \mathrm{NN}_{\mathrm{q}}^{\mathrm{g}}\right)$

$\left[\Delta \mathrm{V}^{0}\right]$ is a vector of dimension $\left(\mathrm{N}_{\mathrm{g}} \times 1\right)$

$[\Delta \mathrm{V}]$ is a vector of dimension $\left(\mathrm{N}_{\mathrm{q}}^{\mathrm{X}} \mathrm{x} 1\right)$

$\mathrm{N}_{\mathrm{g}} \quad$ is the total number of generator or PV buses and

$\mathrm{N}_{\mathrm{q}} \quad$ is the total number of load or PQ buses.

Since $\left[\mathrm{B}^{0}\right],[\mathrm{B}]$ and $[\Delta \mathrm{V}]$ are known, the above equation can be solved using the linear model given below as

$$
\text { Minimize } c^{t} \Delta V^{0}
$$

subject to

$$
\begin{aligned}
& {\left[\mathrm{B}^{0}\right]\left[\Delta \mathrm{V}^{0}\right]=-[\mathrm{B}][\Delta \mathrm{V}]} \\
& \Delta \mathrm{V}^{0} \leq \text { Limit }
\end{aligned}
$$

where $c^{t}$ is the transpose of the cost function vector and Limit is equal to $\left(V_{\max }-V^{0}\right)$ or 
$\left(\mathrm{V}_{0}-\mathrm{V}_{\text {min }}\right)$ depending on the voltage problem and $\mathrm{V}^{0}$ is the base case load flow voltage magnitudes.

The MRBTS can be used as an example to illustrate the model. This system has 3 load buses and 2 generator buses i.e. for this system

$$
\begin{array}{ll}
\mathrm{N}_{\mathrm{g}}=2 & \text { bus } 1 \text { and } 2 \\
\mathrm{~N}_{\mathrm{q}}=3 & \text { bus } 3,4 \text { and } 5 .
\end{array}
$$

Let the cost function be defined as

$$
c^{t}=\left[\begin{array}{ll}
1 & 1
\end{array}\right] \text {. }
$$

The model for this system is therefore,

$$
\text { Minimize } c^{t} \Delta V^{0}
$$

subject to

$$
\begin{aligned}
& \mathrm{B}_{11}{ }^{0} \Delta \mathrm{V}_{1}{ }^{0}+\mathrm{B}_{12}{ }^{0} \Delta \mathrm{V}_{2}{ }^{0}=\mathrm{B}_{11} \Delta \mathrm{V}_{3}+\mathrm{B}_{12} \Delta \mathrm{V}_{4}+\mathrm{B}_{13} \Delta \mathrm{V}_{5} \\
& \mathrm{~B}_{21}{ }^{0} \Delta \mathrm{V}_{1}{ }^{0}+\mathrm{B}_{22}{ }^{0} \Delta \mathrm{V}_{2}{ }^{0}=\mathrm{B}_{21} \Delta \mathrm{V}_{3}+\mathrm{B}_{22} \Delta \mathrm{V}_{4}+\mathrm{B}_{23} \Delta \mathrm{V}_{5} \\
& \mathrm{~B}_{31}{ }^{0} \Delta \mathrm{V}_{1}{ }^{0}+\mathrm{B}_{32}{ }^{0} \Delta \mathrm{V}_{2}{ }^{0}=\mathrm{B}_{31} \Delta \mathrm{V}_{3}+\mathrm{B}_{32} \Delta \mathrm{V}_{4}+\mathrm{B}_{33} \Delta \mathrm{V}_{5} \\
& \Delta \mathrm{V}_{1}{ }^{0} \leq \text { Limit }_{1} \\
& \Delta \mathrm{V}_{2}{ }^{0} \leq \text { Limit }
\end{aligned}
$$

In order to check the effectiveness of the model, the MRBTS load data has been modified as shown in Table 5.1 with all the other values unchanged as shown in Appendix A. The

Table 5.1: Modified load and voltage limits data for MRBTS

\begin{tabular}{ccccc}
\hline Bus & $\mathrm{P}_{\mathrm{L}}$ & $\mathrm{Q}_{\mathrm{L}}$ & $\mathrm{V}_{\min }$ & $\mathrm{V}_{\max }$ \\
\hline & 0.00 & 0.00 & 0.95 & 1.05 \\
2 & 0.20 & 0.07 & 0.95 & 1.05 \\
3 & 0.85 & 0.28 & 0.95 & 1.05 \\
4 & 0.40 & 0.13 & 0.95 & 1.05 \\
5 & 0.401 & 0.13 & 0.95 & 1.05 \\
\hline
\end{tabular}


decoupled load flow result with this data set is shown in Table 5.2. It can be noted from Table 5.2 that bus voltage magnitudes at buses 3 and 4 are within acceptable limits but the voltage magnitude at bus 5 is below the lower specified limit. The change in load bus

Table 5.2: Decoupled load flow results

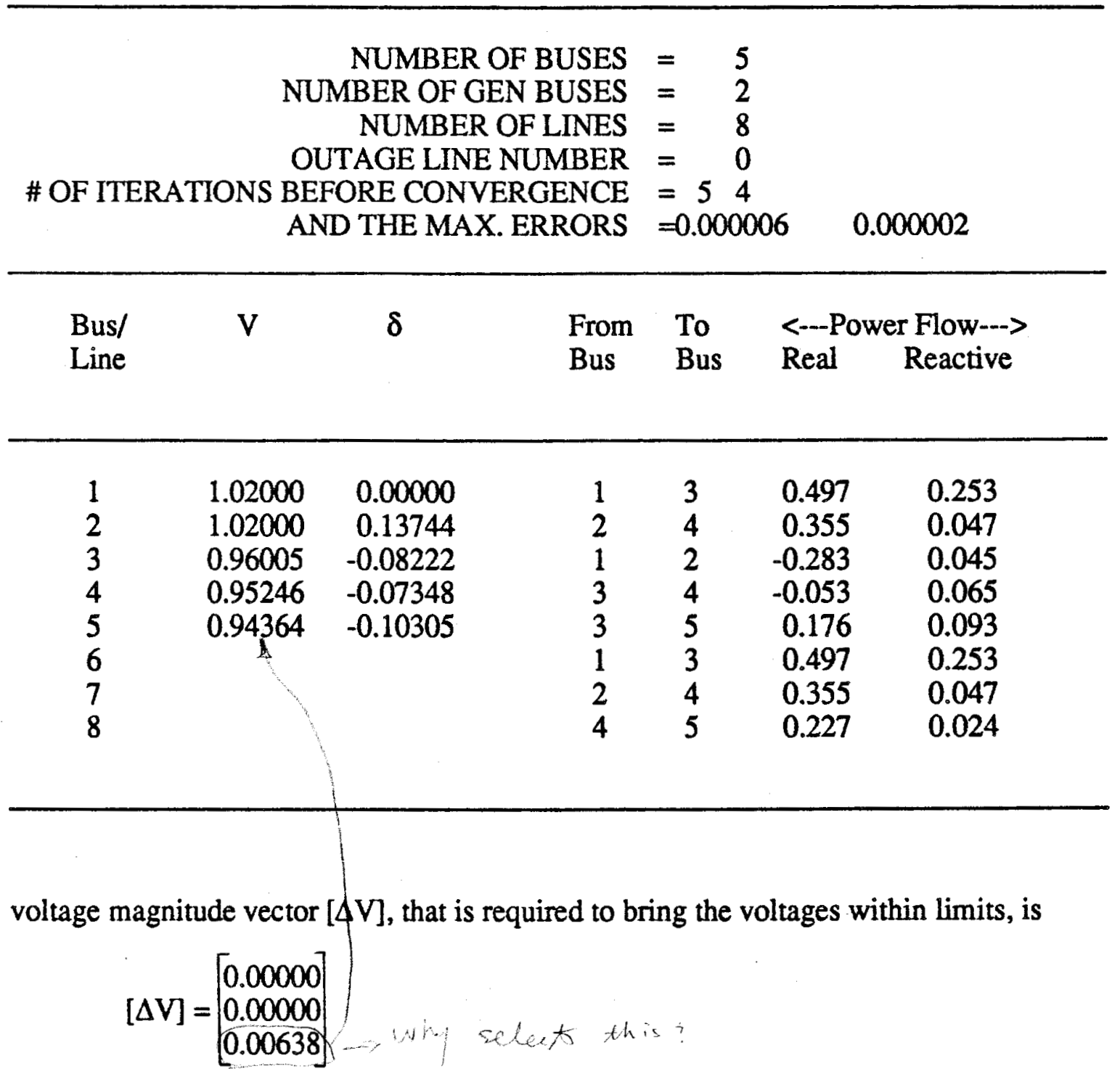

for buses 3,4 and 5 respectively. Substituting the values of $[\Delta V]$ for $V_{3}, V_{4}$ and $V_{5}$ and using other numerical values in Equation 5.4, the model can be given as 
subject to

$$
\text { Minimize } \Delta \mathrm{V}_{1}{ }^{0}+\Delta \mathrm{V}_{2}{ }^{0}
$$

$$
\begin{aligned}
& 11.111 \Delta \mathrm{V}_{1}{ }^{0}+0.000 \Delta \mathrm{V}_{2}{ }^{0}=0.053 \\
& 0.000 \Delta \mathrm{V}_{1}{ }^{0}+3.333 \Delta \mathrm{V}_{2}{ }^{0}=0.053 \\
& 0.000 \Delta \mathrm{V}_{1}{ }^{0}+0.000 \Delta \mathrm{V}_{2}{ }^{0}=0.000 \\
& \Delta \mathrm{V}_{1}{ }^{0} \leq 0.03 \\
& \Delta \mathrm{V}_{2}{ }^{0} \leq 0.03
\end{aligned}
$$

Solving the above model with the upper bounding algorithm explained in Chapter 4, the change in generator bus voltage vector, $\left[\Delta \mathrm{V}^{0}\right]$, that is required to correct the voltage magnitude deviation of bus 5 is

$$
\left[\Delta \mathrm{V}^{0}\right]=[0.004770 .01590]^{\mathrm{T}}
$$

This result indicates that, the voltage magnitudes of buses 1 and 2 have to be increased by 0.00477 and 0.01590 p.u. respectively to bring the voltage magnitude of bus 5 within limits. The decoupled load flow results with this new $\left[\mathrm{V}^{0}\right]$ are shown in Table 5.3. It can be seen that by increasing only the voltage magnitude of bus 1 by $\Delta \mathrm{V}_{1}^{0}=0.0047$, it is not possible to bring the voltage magnitude of bus 5 within the lower limit of 0.95 p.u.. When both voltage magnitudes of buses 1 and 2 are increased by $\Delta \mathrm{V}_{1}{ }^{0}$ and $\Delta \mathrm{V}_{2}{ }^{0}$ respectively, the voltage magnitude of bus 5 becomes greater than the lower acceptable limit.

The model explained above causes difficulties when there are more than one bus violating voltage magnitude limits. Using the above model might therefore give unsatisfactory results for such situations. The MRBTS load data is modified again as shown in Table 5.4 to consider this effect. The corresponding load flow result is shown in Table 5.5. In this case all the load bus voltage magnitudes i.e. at buses 3, 4 and 5 exceeded their limits of 1.05 p.u.. The change in generator bus voltage magnitudes, $\left[\Delta \mathrm{V}^{0}\right]$, obtained using the model explained before is

$$
\left[\Delta \mathrm{V}^{0}\right]=\left[\begin{array}{ll}
0.00958 & 0.02023
\end{array}\right]^{\mathrm{T}} \text {. }
$$

With voltages $\mathrm{V}_{1}{ }^{0}$ and $\mathrm{V}_{2}{ }^{0}$ decreased by $\Delta \mathrm{V}_{1}{ }^{0}$ and $\Delta \mathrm{V}_{2}{ }^{0}$ respectively, the decoupled load flow result is shown in Table 5.6. It can be seen that the result is unsatisfactory as all the 
Table 5.3: Decoupled load flow results with new voltages

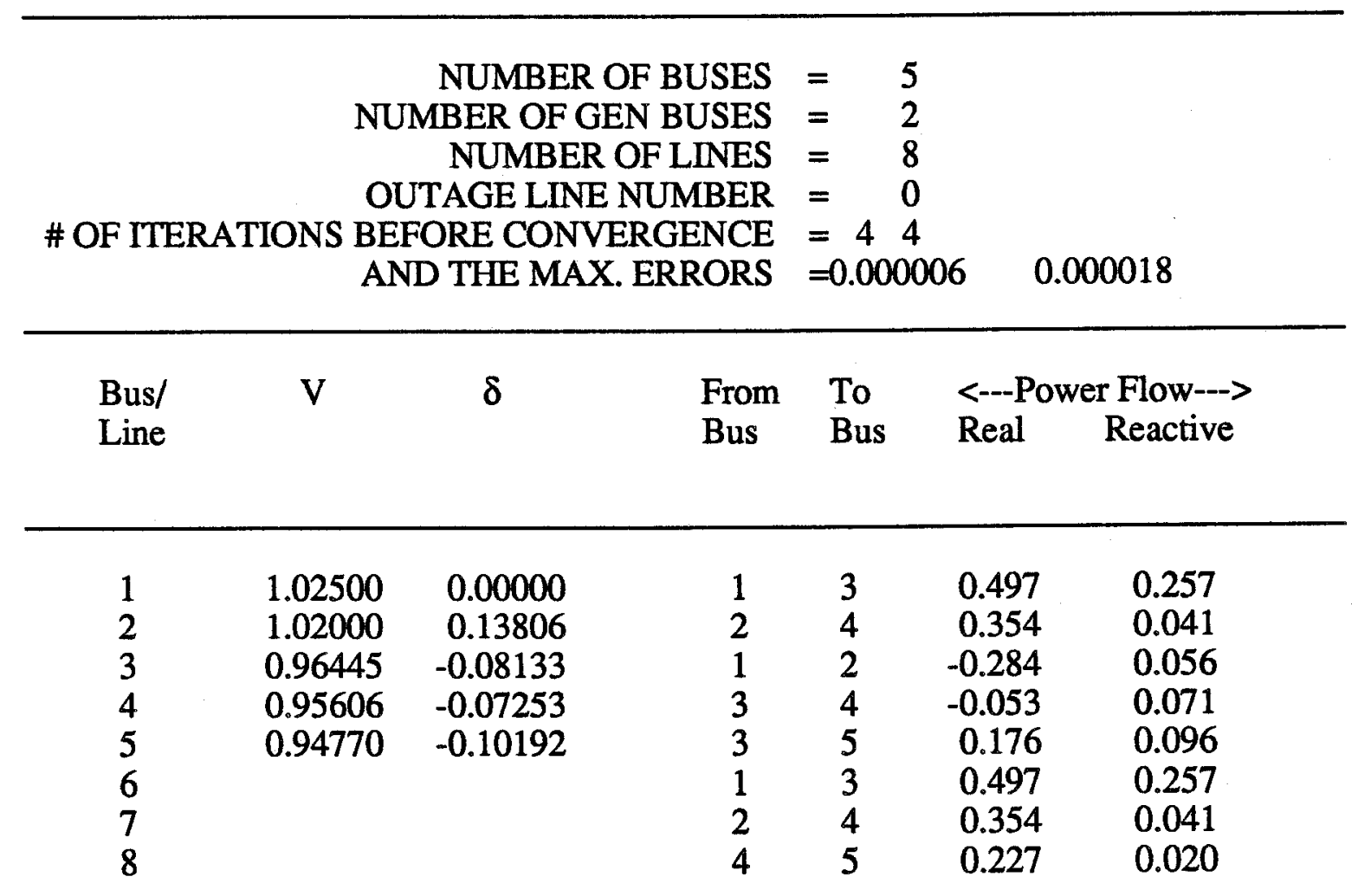

Result with both voltage changed

\# OF ITERATIONS BEFORE CONVERGENCE $=44$

AND THE MAX. ERRORS $=0.000003 \quad 0.000012$

$\begin{array}{rrrrrrr}1 & 1.02500 & 0.00000 & 1 & 3 & 0.495 & 0.237 \\ 2 & 1.03600 & 0.13176 & 2 & 4 & 0.356 & 0.056 \\ 3 & 0.96803 & -0.08137 & 1 & 2 & -0.281 & 0.020 \\ 4 & 0.96261 & -0.07301 & 3 & 4 & -0.055 & 0.048 \\ 5 & 0.95284 & -0.10191 & 3 & 5 & 0.175 & 0.084 \\ 6 & & & 1 & 3 & 0.495 & 0.237 \\ 7 & & & 2 & 4 & 0.356 & 0.056 \\ 8 & & & 4 & 5 & 0.228 & 0.032\end{array}$


Table 5.4: Modified load and voltage limit data for MRBTS

\begin{tabular}{ccccc}
\hline Bus & $\mathrm{P}_{\mathrm{L}}$ & $\mathrm{Q}_{\mathrm{L}}$ & $\mathrm{V}_{\min }$ & $\mathrm{V}_{\max }$ \\
\hline 1 & 0.00 & 0.00 & 0.95 & 1.05 \\
2 & 0.20 & -0.07 & 0.95 & 1.05 \\
3 & 0.85 & -0.28 & 0.95 & 1.05 \\
4 & 0.40 & -0.13 & 0.95 & 1.05 \\
5 & 0.401 & -0.13 & 0.95 & 1.05 \\
\hline
\end{tabular}

Table 5.5: Decoupled load flow results for above data

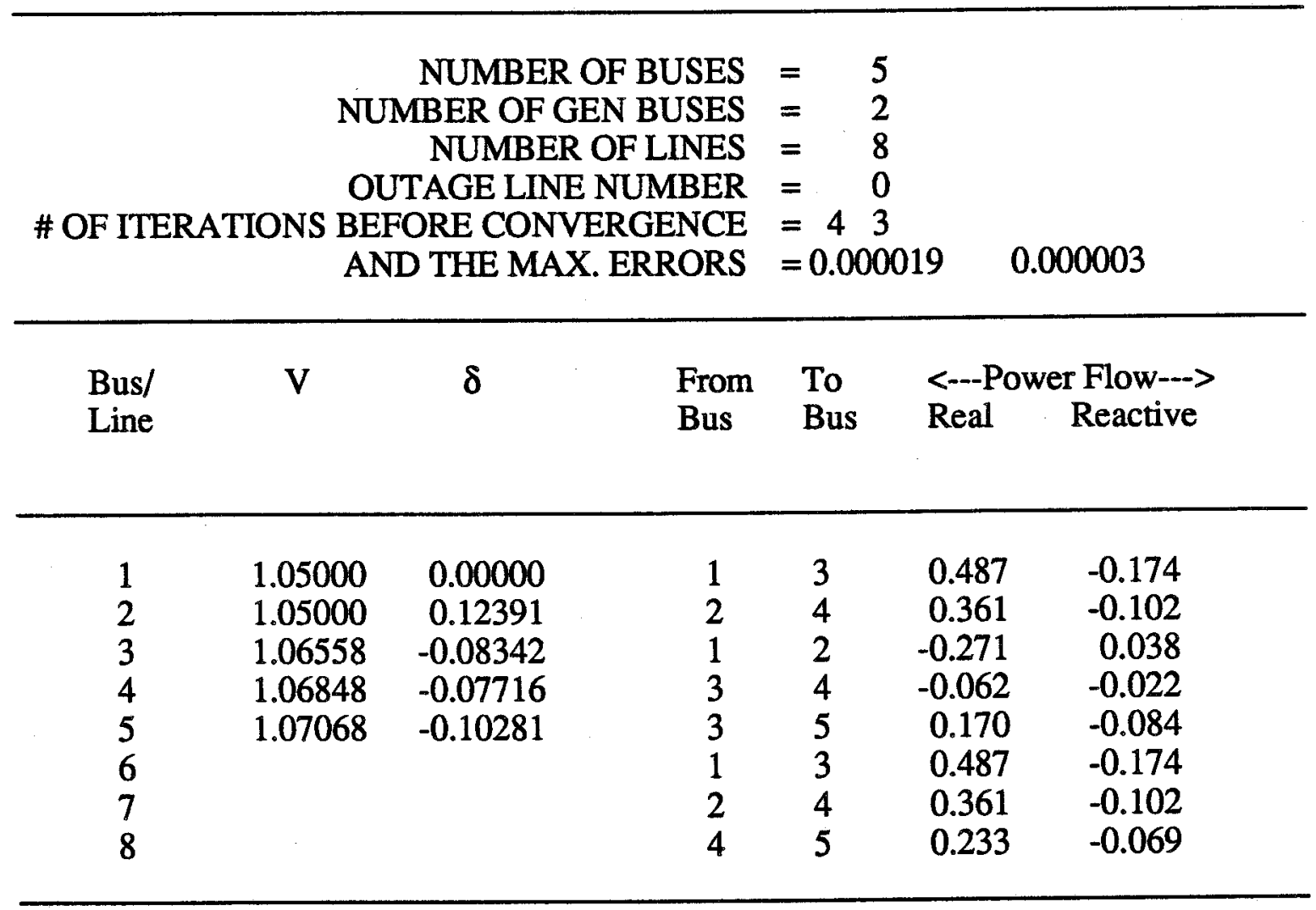

load bus voltages are still greater than the upper limits. This drawback of the above model can be removed by: 
Table 5.6: Decoupled load flow results with modified voltages

\begin{tabular}{|c|c|c|c|c|c|c|}
\hline \multicolumn{4}{|c|}{$\begin{array}{c}\text { NUMBER OF BUSES } \\
\text { NUMBER OF GEN BUSES } \\
\text { NUMBER OF LINES } \\
\text { OUTAGE LINE NUMBER } \\
\text { \# OF ITERATIONS BEFORE CONVERGENCE } \\
\text { AND THE MAX. ERRORS }\end{array}$} & \multicolumn{2}{|c|}{$\begin{array}{l}=5 \\
=\quad 2 \\
=\quad 8 \\
=\quad 0 \\
=43 \\
=0.000013\end{array}$} & 000001 \\
\hline $\begin{array}{l}\text { Bus/ } \\
\text { Line }\end{array}$ & V & $\delta$ & $\begin{array}{l}\text { From } \\
\text { Bus }\end{array}$ & $\begin{array}{l}\text { To } \\
\text { Bus }\end{array}$ & $\begin{array}{l}<-- \text { Po } \\
\text { Real }\end{array}$ & $\begin{array}{c}\text { er Flow---> } \\
\text { Reactive }\end{array}$ \\
\hline $\begin{array}{l}1 \\
2 \\
3 \\
4 \\
5 \\
6 \\
7 \\
8\end{array}$ & $\begin{array}{l}1.04000 \\
1.03000 \\
1.05341 \\
1.05439 \\
1.05756\end{array}$ & $\begin{array}{r}0.00000 \\
0.12989 \\
-0.08499 \\
-0.07841 \\
-0.10478\end{array}$ & $\begin{array}{l}1 \\
2 \\
1 \\
3 \\
3 \\
1 \\
2 \\
4\end{array}$ & $\begin{array}{l}3 \\
4 \\
2 \\
4 \\
5 \\
3 \\
4 \\
5\end{array}$ & $\begin{array}{r}0.489 \\
0.361 \\
-0.272 \\
-0.060 \\
0.171 \\
0.489 \\
0.361 \\
0.232\end{array}$ & $\begin{array}{r}-0.160 \\
-0.109 \\
0.062 \\
-0.005 \\
-0.075 \\
-0.160 \\
-0.109 \\
-0.077\end{array}$ \\
\hline
\end{tabular}

1. selecting $\Delta \mathrm{V}$ for all buses equal to the $\Delta \mathrm{V}$ of that bus which requires maximum voltage change or

2. using both generator and load buses voltage changes $\Delta \mathrm{V}$ as variables.

The results with method one i.e. using the deviations required for all the load buses equal to the $\max \left[\Delta \mathrm{V}_{\mathrm{i}}, \mathrm{i}=1\right.$ to number of load buses] are shown in Tables 5.7 and 5.8. This method has the difficulty that the maximum $\Delta \mathrm{V}$ might be too large for which the solution could be infeasible whereas there may exist a feasible solution. In some cases it might happen that due to the change in maximum $\Delta \mathrm{V}$, some load bus voltages might exceed their limits. These difficulties can be avoided by using the changes in load bus voltage magnitudes as bounded variables in the model similar to the generator bus voltage magnitude changes. The new model for this second method is 
Table 5.7: Voltage magnitude decreases obtained from LP model

\begin{tabular}{cc}
\hline Bus & $\Delta \mathrm{V}$ \\
\hline 1 & 0.02068 \\
2 & 0.02068 \\
\hline
\end{tabular}

Table 5.8: Decoupled load flow results with modified voltages

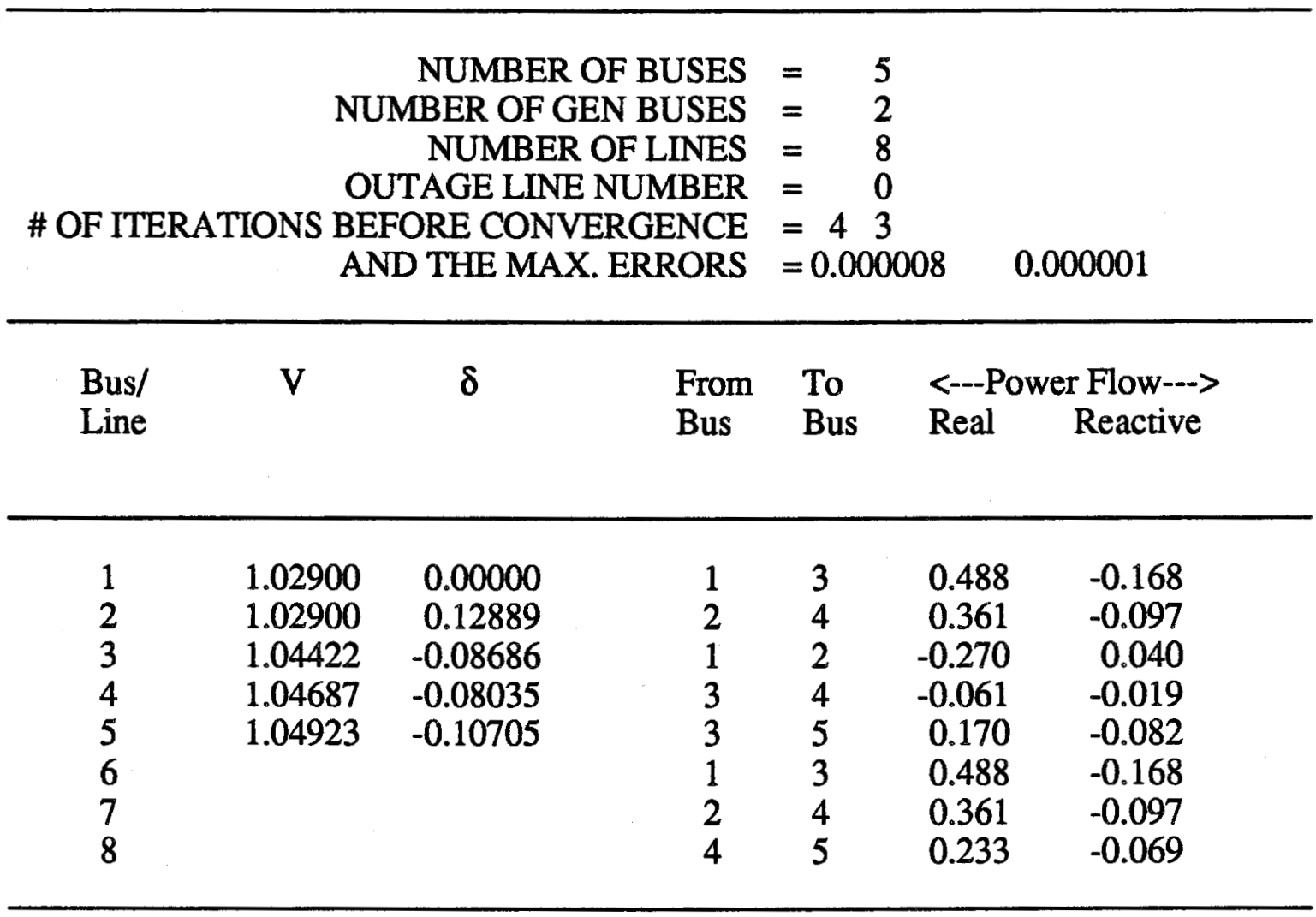


Minimize $c_{1}{ }^{t} \Delta V^{0}$

subject to

$\left[\mathrm{B}^{0}\right]\left[\Delta \mathrm{V}^{0}\right]+[\mathrm{B}][\Delta \mathrm{V}]=0$

$\Delta \mathrm{V}^{0} \leq$ Limit

Lower Limit $\leq \Delta \mathrm{V} \leq$ Upper Limit

The lower limit for the load buses are the required voltage changes to bring the voltages within the limits. The cost vector associated with $[\Delta V]$ is considered equal to zero in this research work. By considering the above model and the data shown in Table 5.4, the required change in generator bus voltage magnitudes, $\left[\Delta \mathrm{V}^{0}\right]$, are

$$
\left[\Delta V^{0}\right]=\left[\begin{array}{lll}
0.02894 & 0.00000
\end{array}\right]^{\mathrm{T}}
$$

The decoupled load flow results have been shown in Table 5.9 after decreasing the generator bus voltage magnitudes. It can be seen that the second alternative requires the change in one bus voltage magnitude which is less than that required in the first method where a total change of $(0.02068+.02068)$ is needed. Similarly for the data set shown in Table 5.1, the required changes with the second alternative are

$$
\left[\Delta \mathrm{V}^{0}\right]=\left[\begin{array}{ll}
0.0088 & 0.0000
\end{array}\right]^{\mathrm{T}}
$$

The decoupled load flow result after increasing the generator bus voltage magnitude at bus 1 is shown in Table 5.10. The second alternative requires less voltage change compared to the model expressed by Equation 5.3 where a total change of $(0.00477+.01590)$ is required. The second alternative, therefore, requires smaller changes in generator bus voltage magnitudes to correct the load bus voltage magnitudes by a desired amount and is used in further studies.

\subsection{CONSIDERATION OF GENERATOR MVAR LIMITS}

The consideration of generator MVAR limits was not included in the constraint set described in Chapter 4. These cosiderations can however be included in the LP model solution for voltage correction. Due to the change in voltage profile, the generator reactive power $\mathrm{Q}$ may exceed its limits. This can be avoided by including constraints for the generator buses in the LP model discussed earlier. The constraints can be developed by considering Equation 5.1 for the generator buses as 
Table 5.9: Decoupled load flow results with modified voltages

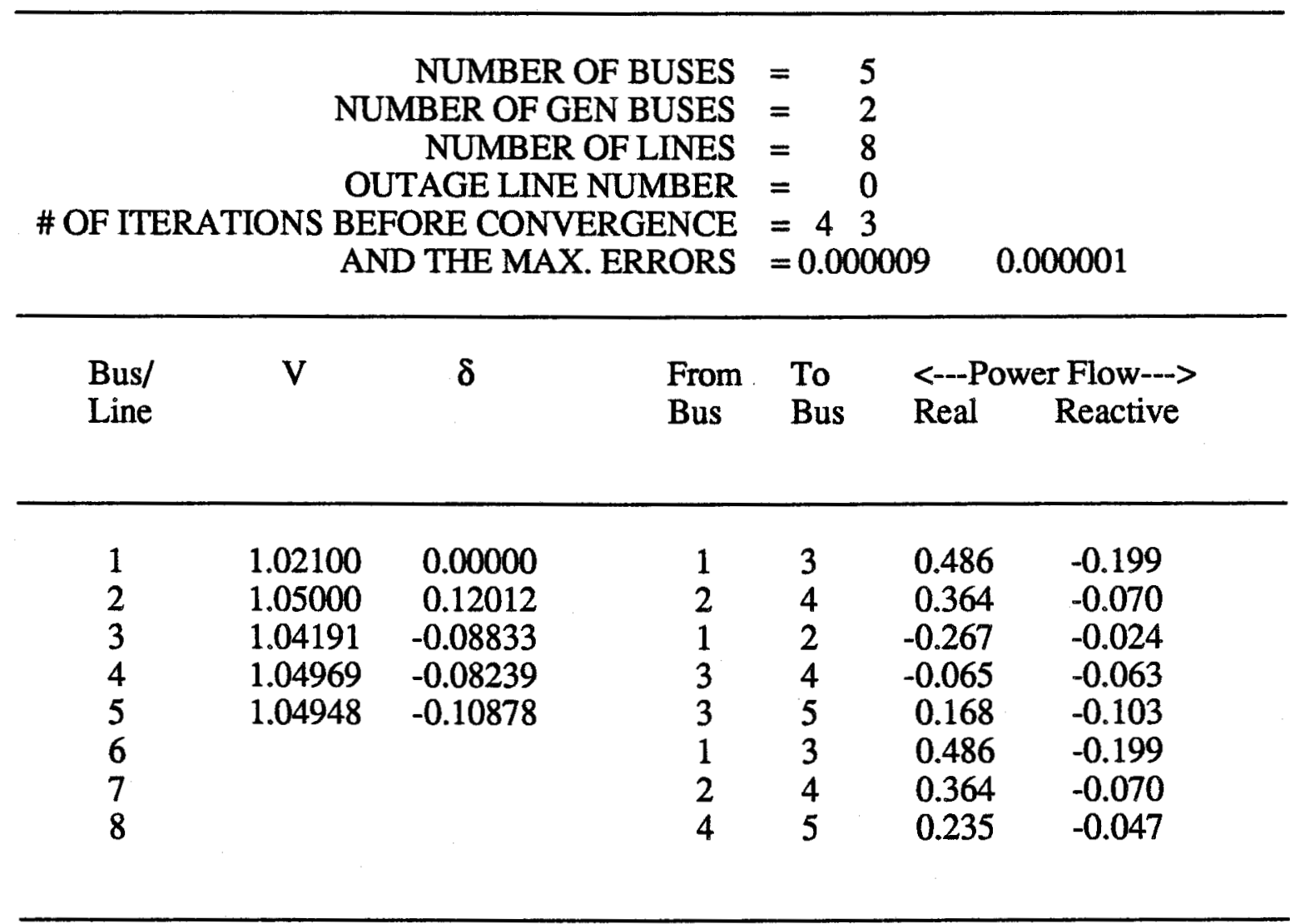

$$
[\mathrm{B}][\mathrm{V}]+\left[\mathrm{B}^{0}\right]\left[\mathrm{V}^{0}\right]=-\left[\mathrm{Q}_{\mathrm{g}} / \mathrm{V}\right]
$$

For changes in voltage magnitudes, let the $\left[\mathrm{Q}_{\mathbf{g}}\right]$ vector be changed to

$$
\left[\mathrm{Q}_{\mathrm{g}}{ }^{\prime}\right]=\left[\mathrm{Q}_{\mathrm{g}}\right]+\left[\Delta \mathrm{Q}_{\mathrm{g}}\right]
$$

Therefore, Equation 5.5 can be rewritten as

$$
\begin{aligned}
{[\mathrm{B}][\mathrm{V}+\Delta \mathrm{V}]+\left[\mathrm{B}^{0}\right]\left[\mathrm{V}^{0}+\Delta \mathrm{V}^{0}\right] } & =-\left[\mathrm{Q}_{\mathrm{g}}{ }^{\prime} / \mathrm{V}\right] \\
& =-\left[\left(\mathrm{Q}_{\mathrm{g}}+\Delta \mathrm{Q}_{\mathrm{g}}\right) / \mathrm{V}\right] \\
& \approx-\left[\mathrm{Q}_{\mathrm{g}} / \mathrm{V}+\Delta \mathrm{Q}_{\mathrm{g}}\right]
\end{aligned}
$$

Simplification with the assumption that $\left[\mathrm{Q}_{\mathrm{g}} / \mathrm{V}\right]$ remains constant gives

$$
[B][\Delta V]+\left[B^{0}\right]\left[\Delta V^{0}\right]=-\left[\Delta Q_{g}\right]
$$


Table 5.10: Decoupled load flow results with modified voltages

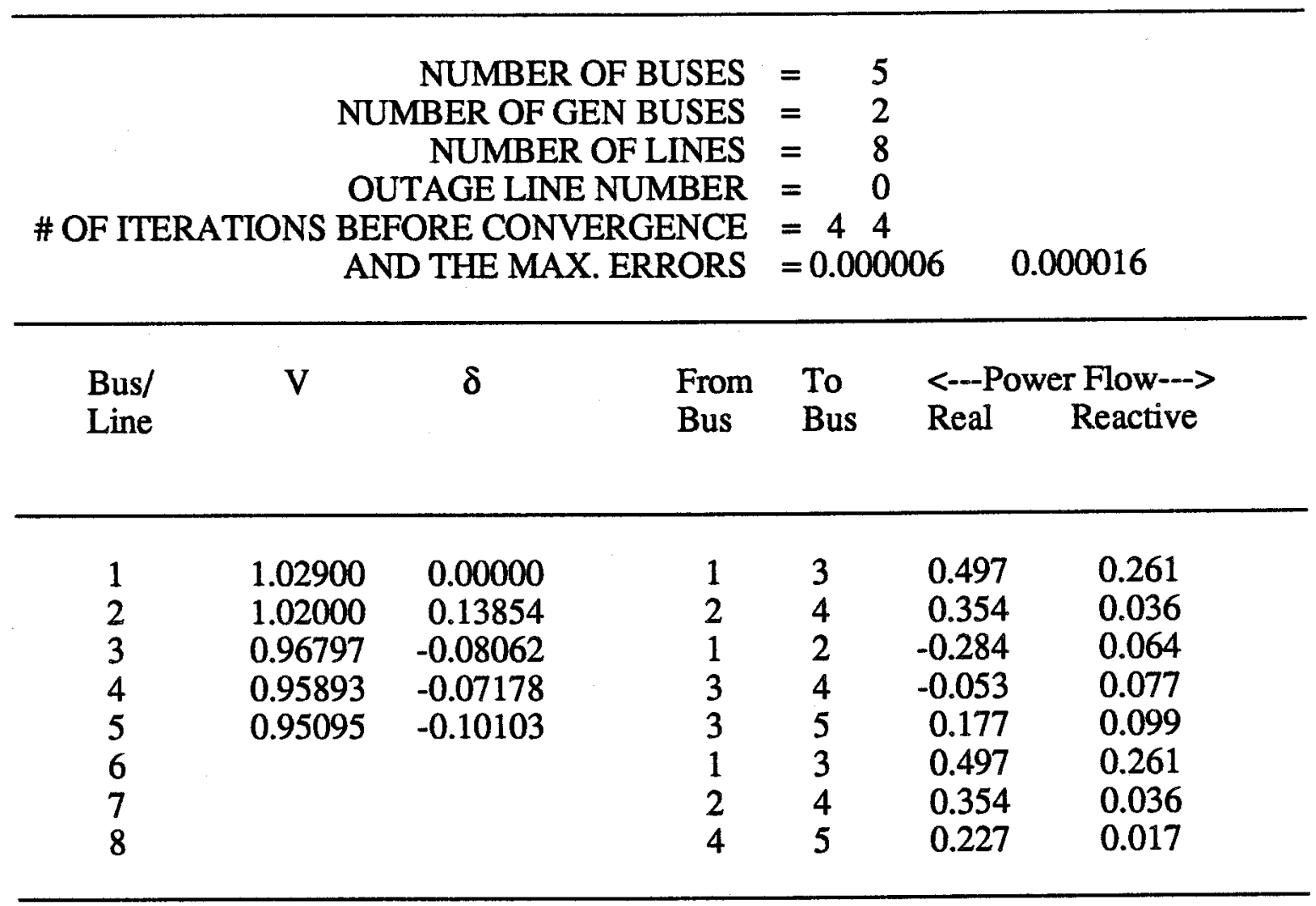

In the above model $\Delta \mathrm{Q}_{\mathrm{g}}$ can be considered as a variable which has a limit that can be found as

$$
\begin{aligned}
& \mathrm{Q}_{\mathrm{g}}^{\min } \leq \mathrm{Q}_{\mathrm{g}}{ }^{\prime} \leq \mathrm{Q}_{\mathrm{g}}^{\max } \\
& \text { or, } \mathrm{Q}_{\mathrm{g}}{ }^{\min } \leq \mathrm{Q}_{\mathrm{g}}+\Delta \mathrm{Q}_{\mathrm{g}} \leq \mathrm{Q}_{\mathrm{g}} \max \\
& \text { or, } \mathrm{Q}_{\mathrm{g}}{ }^{\min } \mathrm{Q}_{\mathrm{g}} \leq \Delta \mathrm{Q}_{\mathrm{g}} \leq \mathrm{Q}_{\mathrm{g}}{ }^{\text {max }}-\mathrm{Q}_{\mathrm{g}}
\end{aligned}
$$

where $Q_{g}$ is known from the base case load flow solution or can be found from the contingency cases in which the updated voltages were found by the approximate selection method. Several studies similar to those conducted for the second alternative method were conducted and the results were found to be similar to those reported earlier since the generator $\mathrm{Q}$ limits do not exceed the values before voltage changes. 


\subsection{REACTIVE LOAD CURTAILMENT BY THE LP MODEL}

Due to a contingency, it might not be possible in some situations to bring the load bus voltage magnitudes within limits by changing only the generator bus voltage magnitudes. The load bus voltage magnitudes, however, can be brought within limits by improving the power factors at appropriate load buses. The improvement in power factors can be considered to be accomplished by cutting the reactive loads at the load buses. The solution will therefore be infeasible in the above model, when there is a requirement for Q-load curtailment. The original LP model can be structured such that the artificial variables added to each constraint will be the amount of reactive load curtailed.

Consider the case where the Q-load curtailment vector of the load buses is $[\Delta \mathrm{Q}]$ which is required in addition to generator bus voltage magnitude changes, Equation 5.1 can be written with the assumption noted earlier as

$$
[\mathrm{B}][\mathrm{V}+\Delta \mathrm{V}]+\left[\mathrm{B}^{0}\right]\left[\mathrm{V}^{0}+\Delta \mathrm{V}^{0}\right]=[\mathrm{Q}-\Delta \mathrm{Q}]
$$

or,

$$
[\mathrm{B}][\Delta \mathrm{V}]+\left[\mathrm{B}^{0}\right]\left[\Delta \mathrm{V}^{0}\right]+[\Delta \mathrm{Q}]=0
$$

This equation is similar to the equation of the LP model noted earlier with the $\Delta \mathrm{Q}$ term added. The coefficient of $\Delta \mathrm{Q}$ is unity and hence the $\mathrm{Q}$-load shed $\Delta \mathrm{Q}$, can be represented by the artificial variables added to the above LP model by putting a high penalty cost for $\Delta \mathrm{Q}$. These variables will represent the amount of Q-load that has to be shed in order to bring the voltages within the limits. The load model shown in Table 5.11 for the MRBTS has been used to illustrate the amount of reactive power curtailment. The decoupled load flow results are shown in Table 5.12. The corresponding results from the LP model are

$$
\begin{aligned}
& {\left[\Delta \mathrm{V}^{0}\right]=\left[\begin{array}{ll}
0.03 & 0.03
\end{array}\right]^{\mathrm{T}} \text { and }} \\
& {[\mathrm{Q} \text {-shed }]=\left[\begin{array}{lll}
0.00 & 0.00 & 0.16
\end{array}\right]^{\mathrm{T}}}
\end{aligned}
$$

The decoupled load flow results with [ $\left.\mathrm{V}^{0}\right]$ changes and without cutting reactive load are shown in Table 5.13 and considering voltage magnitude changes as well as reactive load shedding are shown in Table 5.14. It can be seen from these tables that changes only in 
Table 5.11: Modified load and voltage limit data for MRBTS

\begin{tabular}{ccccccc}
\hline Bus & $\mathrm{P}_{\mathrm{L}}$ & $\mathrm{Q}_{\mathrm{L}}$ & $\mathrm{V}_{\min }$ & $\mathrm{V}_{\max }$ & $\mathrm{Q}_{\min }$ & $\mathrm{Q}_{\max }$ \\
\hline 1 & 0.00 & 0.00 & 0.97 & 1.05 & -0.8 & 0.8 \\
2 & 0.20 & 0.10 & 0.97 & 1.05 & -0.4 & 0.4 \\
3 & 0.85 & 0.41 & 0.97 & 1.05 & - & - \\
4 & 0.40 & 0.19 & 0.97 & 1.05 & - & - \\
5 & 0.401 & 0.19 & 0.97 & 1.05 & - & - \\
\hline
\end{tabular}

Table 5.12: Decoupled load flow results with modified voltages

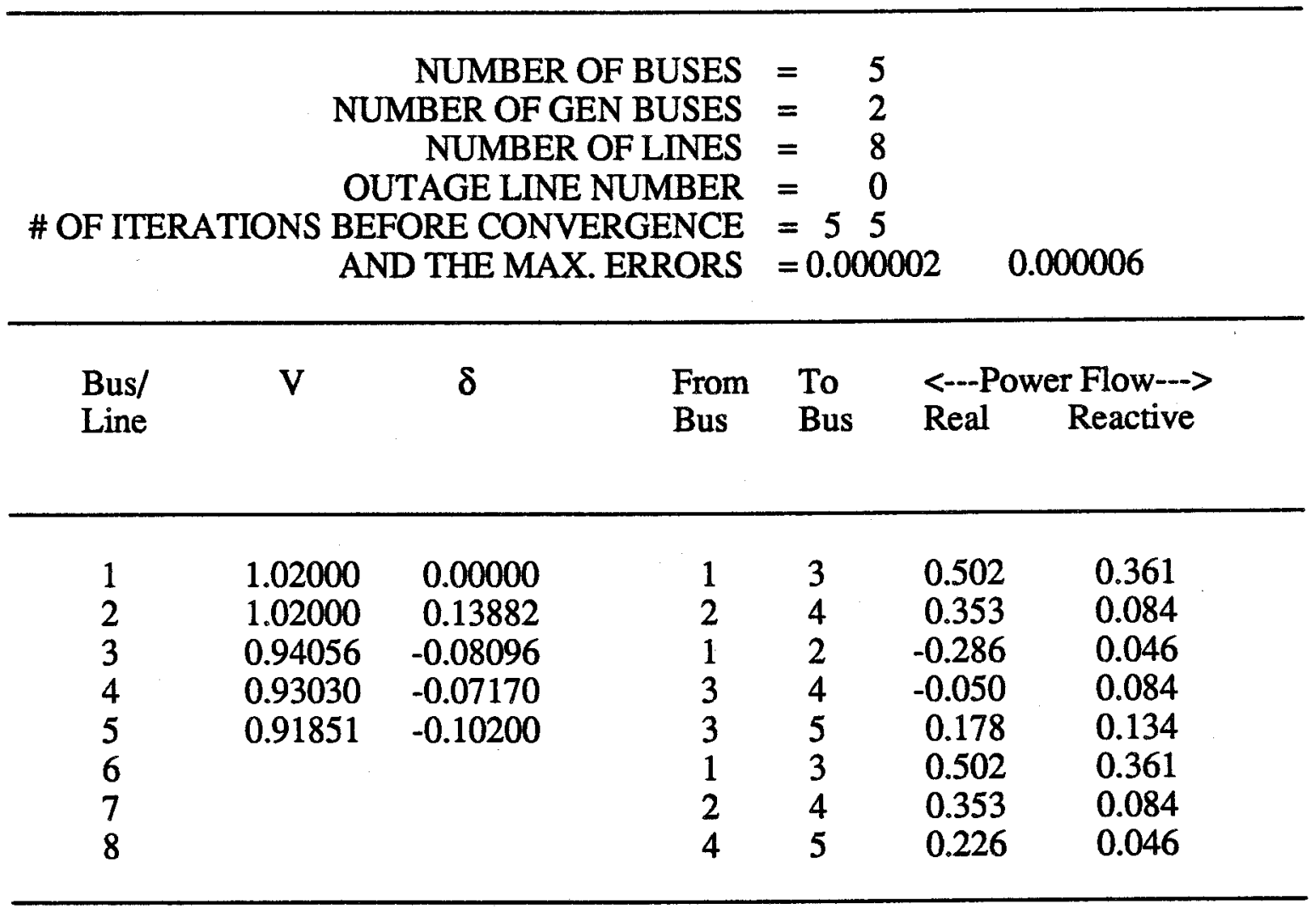

generator bus voltage magnitudes is not sufficient to bring all the load bus voltage magnitudes within the specified limits. The $Q$ of load bus 5 has to be reduced by 0.16 p.u. to bring the voltages back to the acceptable limits. 
Table 5.13: Decoupled load flow results with modified voltages

\begin{tabular}{|c|c|c|c|c|c|c|}
\hline \multicolumn{4}{|c|}{$\begin{array}{r}\text { NUMBER OF BUSES } \\
\text { NUMBER OF GEN BUSES } \\
\text { NUMBER OF LINES } \\
\text { OUTAGE LINE NUMBER } \\
\text { \# OF ITERATIONS BEFORE CONVERGENCE } \\
\text { AND THE MAX. ERRORS }\end{array}$} & \multicolumn{2}{|c|}{ 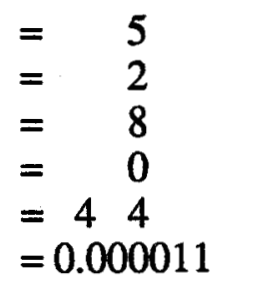 } & 00011 \\
\hline $\begin{array}{l}\text { Bus/ } \\
\text { Line }\end{array}$ & V & $\delta$ & $\begin{array}{l}\text { From } \\
\text { Bus }\end{array}$ & $\begin{array}{l}\text { To } \\
\text { Bus }\end{array}$ & \multicolumn{2}{|c|}{ <---Power Flow---> } \\
\hline $\begin{array}{l}1 \\
2 \\
3 \\
4 \\
5 \\
6 \\
7 \\
8\end{array}$ & $\begin{array}{l}1.05000 \\
1.05000 \\
0.97439 \\
0.96499 \\
0.95346\end{array}$ & $\begin{array}{r}0.00000 \\
0.13077 \\
-0.07580 \\
-0.06706 \\
-0.09530\end{array}$ & $\begin{array}{l}1 \\
2 \\
1 \\
3 \\
3 \\
1 \\
2 \\
4\end{array}$ & $\begin{array}{l}3 \\
4 \\
2 \\
4 \\
5 \\
3 \\
4 \\
5\end{array}$ & $\begin{array}{r}0.499 \\
0.354 \\
-0.285 \\
-0.052 \\
0.177 \\
0.499 \\
0.354 \\
0.226\end{array}$ & $\begin{array}{l}0.351 \\
0.076 \\
0.043 \\
0.080 \\
0.131 \\
0.351 \\
0.076 \\
0.046\end{array}$ \\
\hline
\end{tabular}

\subsection{STUDY RESULTS}

The probabilistic indices for different operating states have been calculated using the two test systems described earlier. The results presented in this section considered up to the 4th level of generator outages, 3rd level of line outages and the 3rd level of generator and line combination outages. In these studies, the Q-load curtailment is considered to have the same penalty as the real load curtailment. This means that whenever there is a curtailment of Q-load to bring the voltages within the limits, the system state is in the extreme emergency state as in the case of real load curtailment. 
Table 5.14: Decoupled load flow results with modified voltages and $Q$

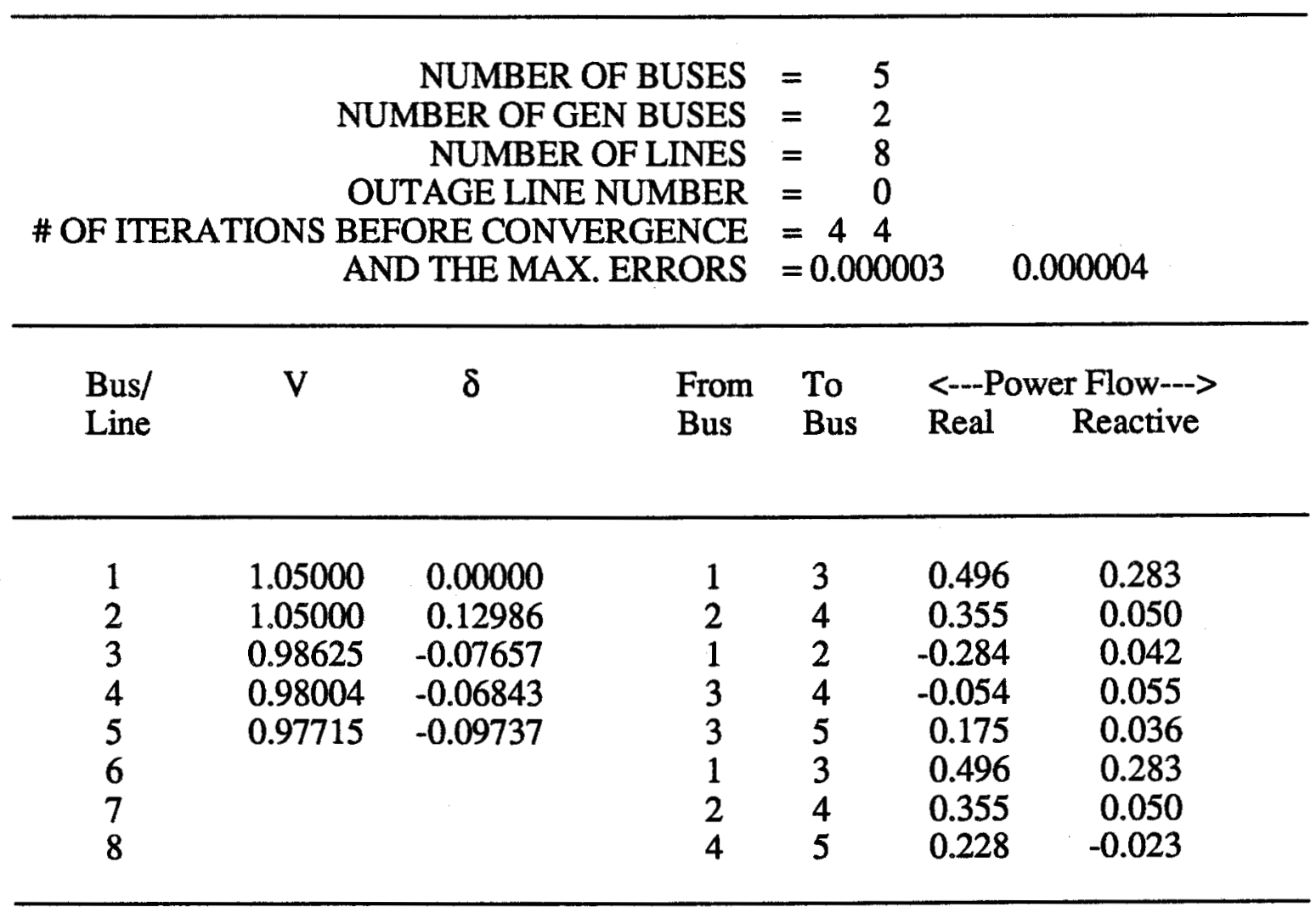

\subsubsection{Results For The MRBTS}

The annualized indices for this system considering only the voltage problem is shown in Table 5.15. It can be seen from this table that the probability and frequency of the normal state is greater than zero for this test system which means that there is no single level contingency which creates a voltage problem. The probability and frequency of the extreme emergency state are higher than the probability and frequency of the emergency state because in most cases, especially high level generator outages, load curtailments are required at the time of peak load to overcome the problem. 
Table 5.15: Probability and frequency of different operating states - MRBTS, considering only voltage problems

\begin{tabular}{llr}
\hline $\begin{array}{llr}\text { System } \\
\text { State }\end{array}$ & Probability & Frequency \\
& & \\
\hline Normal & 0.845140 & 70.721707 \\
Alert & 0.146305 & 38.700713 \\
Emergency & 0.000000 & 0.000000 \\
Ext. Emergency & 0.008306 & 3.643461 \\
No problem & 0.000224 & 0.311945 \\
\hline Total considered & 0.999975 & \\
\hline
\end{tabular}

\subsubsection{Results For The IEEE-RTS}

The annualized indices for this test system at a system load level of $2850 \mathrm{MW}$ are shown in Table 5.16. The first level outage of line 11 and its associated higher level outages (combined line 11 and higher numbered lines plus line 11 and generators) are ignored. The reason for this is explained in Chapter 4. As can be seen from Table 5.16, the probability of the normal state is zero for the IEEE-RTS when a voltage problem is considered as a system problem. This is because at this peak load when the bus voltages for the " $P-V$ "' buses are set equal to 1.02 p.u. there are 4 single level outages (lines 4, 7, 10 and 27) which create voltage problems and require some var injection or the "P-V" bus voltages raised to overcome this difficulty. If only line overload is considered as the system problem, then the probability of the normal state is greater than zero as seen before which means that no single level outages create line overload problems. The effect of adjusting the "P-V" bus voltages on the results will be discussed later. The probability and frequency of the extreme emergency state are higher than those of the emergency state. This is because of the fact that most of the problem creating contingencies, especially the higher level generator outages, require load curtailment at the peak load to overcome the problem. This may not be true for other load levels. The 
Table 5.16: Probability and frequency of different operating states - IEEE-RTS, considering only voltage problems

\begin{tabular}{lrr} 
System & Probability & Frequency \\
State & & \\
& & \\
Normal & 0.000000 & 0.000000 \\
Alert & 0.876821 & 396.686142 \\
Emergency & 0.001259 & 0.849392 \\
Ext. Emergency & 0.087197 & 54.855049 \\
No problem & 0.020329 & 20.409108 \\
& & \\
& & \\
Total considered & 0.985606 & \\
* Outage of line 11 ignored. & \\
\hline
\end{tabular}

'probability of no problem' is the sum of the probabilities of those third or fourth level contingencies which do not create voltage problems and since a decision can not be taken for these contingencies as to which state they belong, they are added up to represent the probability of no problem creating contingencies as before. In this case the system has no normal state due to the four single level line outages and therefore the 'probability of no problem' could be added to the alert state.

The annual indices for the two test systems are shown in Tables 5.17 to 5.20 considering only the voltage problem.

\subsection{TOTAL CONSTRAINT SET AND ANNUAL INDICES}

The reliability indices obtained by considering the overall constraint set discussed earlier are presented in Tables 5.21 and 5.22 for the two test systems respectively. These indices were calculated at a system load equal to the peak load throughout the year. The results shown in Tables 5.21 and 5.22, therefore, are pessimistic as the load is not equal to the peak load throughout the year. The annual indices were calculated using a seven step load model. The load steps and the probabilities of each load step are presented in Chapter 4. The annual indices are shown in Tables 5.23 through 5.26 for the two test 
Table 5.17: Effect of Load variation on the probability of different states MRBTS, considering only voltage problems

\begin{tabular}{rllllll}
\hline $\begin{array}{l}\text { Load } \\
\text { Step }\end{array}$ & Normal & Alert & $\begin{array}{c}\text { Probability of } \\
\text { Emergency }\end{array}$ & $\begin{array}{c}\text { Ext. } \\
\text { Emergency }\end{array}$ & $\begin{array}{c}\text { No } \\
\text { Problem }\end{array}$ \\
\hline & & & & & & \\
\hline $40 \%$ & 0.997500 & 0.002050 & 0.000000 & 0.000001 & 0.000424 \\
$50 \%$ & 0.996382 & 0.003167 & 0.000001 & 0.000001 & 0.000423 \\
$60 \%$ & 0.997409 & 0.002140 & 0.000000 & 0.000003 & 0.000422 \\
$70 \%$ & 0.996479 & 0.003055 & 0.000000 & 0.000024 & 0.000417 \\
$80 \%$ & 0.991949 & 0.007433 & 0.000000 & 0.000186 & 0.000406 \\
$90 \%$ & 0.918070 & 0.079286 & 0.000000 & 0.002263 & 0.000355 \\
$100 \%$ & 0.845140 & 0.146305 & 0.000000 & 0.008306 & 0.000223 \\
annual & 0.985247 & 0.013921 & 0.000000 & 0.000398 & 0.000408 \\
\hline
\end{tabular}

Total probability considered in all cases $=0.999975$.

Table 5.18: Effect of load variation on the frequency of different states - MRBTS, considering only voltage problems

\begin{tabular}{crrrrr}
\hline $\begin{array}{l}\text { Load } \\
\text { Step }\end{array}$ & $\begin{array}{c}\text { Normal } \\
\end{array}$ & Alert & $\begin{array}{c}\text { Frequency of } \\
\text { Emergency }\end{array}$ & $\begin{array}{c}\text { Ext. } \\
\text { Emergency }\end{array}$ & $\begin{array}{c}\text { No } \\
\text { Problem }\end{array}$ \\
\hline $40 \%$ & 110.839036 & 1.984604 & 0.000000 & 0.002703 & 0.551484 \\
$50 \%$ & 109.744775 & 3.076992 & 0.002356 & 0.002774 & 0.550929 \\
$60 \%$ & 110.772076 & 2.051563 & 0.000000 & 0.004012 & 0.550175 \\
$70 \%$ & 110.339883 & 2.474791 & 0.000000 & 0.017024 & 0.546128 \\
$80 \%$ & 108.413930 & 4.313600 & 0.000000 & 0.111856 & 0.538440 \\
$90 \%$ & 87.914728 & 23.937328 & 0.000000 & 1.048018 & 0.477753 \\
$100 \%$ & 70.721707 & 38.700713 & 0.000000 & 3.643461 & 0.311945 \\
annual & 106.986521 & 5.666064 & 0.000533 & 0.188372 & 0.536336 \\
\hline
\end{tabular}


Table 5.19: Eriect of load variation on the probability of different states - IEEERTS, considering only voltage problems

\begin{tabular}{cccccc}
\hline $\begin{array}{l}\text { Load } \\
\text { Step }\end{array}$ & $\begin{array}{c}\text { Normal } \\
\end{array}$ & Alert & $\begin{array}{c}\text { Probability of } \\
\text { Emergency }\end{array}$ & $\begin{array}{c}\text { Ext. } \\
\text { Emergency }\end{array}$ & $\begin{array}{c}\text { No } \\
\text { Problem }\end{array}$ \\
\hline & & & & & \\
\hline $50 \%$ & 0.937949 & 0.006691 & 0.000009 & 0.000003 & 0.040955 \\
$60 \%$ & 0.935922 & 0.008717 & 0.000010 & 0.000003 & 0.040954 \\
$70 \%$ & 0.000000 & 0.944460 & 0.000317 & 0.000004 & 0.040826 \\
$80 \%$ & 0.000000 & 0.943998 & 0.001112 & 0.000004 & 0.040493 \\
$90 \%$ & 0.0000000 & 0.943471 & 0.001066 & 0.001305 & 0.039765 \\
$100 \%$ & 0.000000 & 0.938479 & 0.001067 & 0.011590 & 0.034470 \\
annual & 0.246054 & 0.676821 & 0.001259 & 0.087197 & 0.020329 \\
& & 0.69628 & 0.000640 & 0.002653 & 0.039631 \\
\hline
\end{tabular}

Total probability considered in all cases $=0.985606$.

* Outage of line 11 ignored.

systems. It can be seen from these tables that the probability and the frequency for the emergency state is higher than the corresponding values of the extreme emergency state for lower load levels. The probability of the normal state is zero for the IEEE-RTS as can be seen from Table 5.25, for a load level higher than $50 \%$ of the system peak load. The reason for this was explained earlier.

Several studies have been conducted using different ' $\mathrm{P}-\mathrm{V}$ ' bus voltages in order to demonstrate the effect of the 'P-V' bus voltage settings on the probabilistic indices. These results are given in Tables 5.27 and 5.28 for different load levels. In the case of the $80 \%$ load level, for example, if the 'P-V' bus voltages are set at 1.02 p.u., then there are 3 single level cases (lines 4,10 and 27) found by the approximate selection method, which create a system voltage problem. Therefore, the probability of normal state becomes zero since it cannot withstand single level outage(s) without violation. In the results shown in Tables 5.27 and 5.28, the voltages for all 'P-V' buses are set at 1.03 p.u. except for bus $6(=1.02$ p.u.) and buses 3 and $7(=1.05$ p.u.) for the $80 \%, 90 \%$ and $100 \%$ 
Table 5.20: Effect of load variation on the frequency of different states - IEEERTS, considering only voltage problems

\begin{tabular}{rrrrrr}
\hline $\begin{array}{l}\text { Load } \\
\text { Step }\end{array}$ & \multicolumn{1}{c}{ Normal } & Alert & $\begin{array}{r}\text { Frequency of } \\
\text { Emergency }\end{array}$ & $\begin{array}{c}\text { Ext. } \\
\text { Emergency }\end{array}$ & $\begin{array}{c}\text { No } \\
\text { Problem }\end{array}$ \\
\hline & & & & & \\
$40 \%$ & 429.581364 & 5.399080 & 0.008285 & 0.005229 & 37.805733 \\
$50 \%$ & 428.840265 & 6.139740 & 0.009491 & 0.005229 & 37.804967 \\
$60 \%$ & 0.000000 & 434.762293 & 0.403412 & 0.005779 & 37.628208 \\
$70 \%$ & 0.000000 & 434.452375 & 0.991172 & 0.006389 & 37.349754 \\
$80 \%$ & 0.000000 & 434.088586 & 0.952872 & 0.974026 & 36.784207 \\
$90 \%$ & 0.000000 & 431.227585 & 0.953259 & 7.893155 & 32.725693 \\
$100 \%$ & 0.000000 & 396.686142 & 0.849392 & 54.855049 & 20.409108 \\
annual & 112.735083 & 321.007392 & 0.594022 & 1.763736 & 36.699458 \\
\hline
\end{tabular}

* Outage of line 11 ignored.

Table 5.21: Probability and frequency of different operating states - MRBTS, considering total constraint set

\begin{tabular}{llr}
\hline System & Probability & Frequency \\
State & & \\
& & \\
Normal & 0.813090 & 51.976677 \\
Alert & 0.177980 & 56.966892 \\
Emergency & 0.000137 & 0.207265 \\
Ext. Emergency & 0.008589 & 3.988032 \\
No problem & 0.000179 & 0.238960 \\
& & \\
\hline & & \\
\hline
\end{tabular}

load levels. The probability of the normal state, for these voltage settings are greater than zero since no single level contingency is found to violate the constraint set. The 
Table 5.22: Probability and frequency of different operating states - IEEE-RTS, considering total constraint set

\begin{tabular}{lrr}
\hline System & Probability & Frequency \\
State & & \\
& & \\
Normal & 0.000000 & 0.000000 \\
Alert & 0.876821 & 396.685555 \\
Emergency & 0.001260 & 0.850784 \\
Ext. Emergency & 0.087197 & 54.855049 \\
No problem & 0.020329 & 20.408278 \\
\hline & & \\
Total considered & 0.985606 & \\
* Outage of line 11 ignored. & \\
\hline
\end{tabular}

Table 5.23: Effect of load variation on the probability of different states - MRBTS, considering total constraint set

\begin{tabular}{|c|c|c|c|c|c|}
\hline \multirow{2}{*}{$\begin{array}{l}\text { Load } \\
\text { Step }\end{array}$} & \multicolumn{2}{|c|}{$<---1-$} & \multirow{2}{*}{$\begin{array}{c}\text { Probability of } \\
\text { Emergency }\end{array}$} & \multirow{2}{*}{ Ext. } & \multirow[b]{2}{*}{$\begin{array}{c}\text { No } \\
\text { Problem }\end{array}$} \\
\hline & Normal & Alert & & & \\
\hline $40 \%$ & 0.982089 & 0.017379 & 0.000097 & 0.000001 & 0.000408 \\
\hline $50 \%$ & 0.982068 & 0.017404 & 0.000092 & 0.000001 & 0.000409 \\
\hline $60 \%$ & 0.982061 & 0.017412 & 0.000091 & 0.000003 & 0.000408 \\
\hline $70 \%$ & 0.981151 & 0.018306 & 0.000091 & 0.000024 & 0.000402 \\
\hline $80 \%$ & 0.977488 & 0.021828 & 0.000081 & 0.000186 & 0.000393 \\
\hline $90 \%$ & 0.901276 & 0.095957 & 0.000076 & 0.002341 & 0.000324 \\
\hline $100 \%$ & 0.813090 & 0.177980 & 0.000137 & 0.008589 & 0.000179 \\
\hline annual & 0.969902 & 0.029182 & 0.000089 & 0.000410 & 0.000392 \\
\hline
\end{tabular}

Total probability considered in all cases $=0.999975$.

probabilistic indices indicate that the system states are very dependent on the initial voltage settings. Acceptable operating bus voltage settings are therefore very important 
Table 5.24: Effect of load variation on the frequency of different states - MRBTS, considering total constraint set

\begin{tabular}{rccccc}
\hline $\begin{array}{l}\text { Load } \\
\text { Step }\end{array}$ & $\begin{array}{c}\text { Normal } \\
\end{array}$ & Alert & $\begin{array}{c}\text { Frequency of } \\
\text { Emergency }\end{array}$ & $\begin{array}{c}\text { Ext. } \\
\text { Emergency }\end{array}$ & $\begin{array}{c}\text { No } \\
\text { Problem }\end{array}$ \\
\hline & & & & & \\
\hline $50 \%$ & 96.133540 & 16.542751 & 0.178764 & 0.002703 & 0.520069 \\
$50 \%$ & 96.109281 & 16.574485 & 0.169252 & 0.002774 & 0.522035 \\
$60 \%$ & 96.140157 & 16.545481 & 0.166672 & 0.004249 & 0.521268 \\
$70 \%$ & 95.731747 & 16.944926 & 0.166280 & 0.017278 & 0.517596 \\
$80 \%$ & 94.620309 & 17.986042 & 0.148587 & 0.112103 & 0.510786 \\
$90 \%$ & 73.048313 & 38.622442 & 0.139045 & 1.143518 & 0.424509 \\
$100 \%$ & 51.976677 & 56.966892 & 0.207265 & 3.988032 & 0.238960 \\
annual & 92.641408 & 19.866322 & 0.162082 & 0.203662 & 0.504352 \\
\hline
\end{tabular}

Table 5.25: Effect of load variation on the probability of different states - IEEERTS, considering total constraint set

\begin{tabular}{rccccc}
\hline $\begin{array}{l}\text { Load } \\
\text { Step }\end{array}$ & Normal & Alert & $\begin{array}{c}\text { Probability of } \\
\text { Emergency }\end{array}$ & $\begin{array}{c}\text { Ext. } \\
\text { Emergency }\end{array}$ & $\begin{array}{c}\text { No } \\
\text { Problem }\end{array}$ \\
\hline & & & & & \\
\hline $50 \%$ & 0.937659 & 0.006980 & 0.000009 & 0.000003 & 0.040955 \\
$50 \%$ & 0.935633 & 0.009006 & 0.000010 & 0.000003 & 0.040954 \\
$60 \%$ & 0.000000 & 0.944460 & 0.000321 & 0.000004 & 0.040822 \\
$70 \%$ & 0.000000 & 0.943998 & 0.001115 & 0.000004 & 0.040489 \\
$80 \%$ & 0.000000 & 0.943471 & 0.001070 & 0.001305 & 0.039761 \\
$90 \%$ & 0.000000 & 0.938479 & 0.001071 & 0.011590 & 0.034466 \\
$100 \%$ & 0.000000 & 0.876821 & 0.001260 & 0.087197 & 0.020329 \\
annual & 0.245978 & 0.696704 & 0.000643 & 0.002653 & 0.039629 \\
\hline
\end{tabular}

Total probability considered in all cases $=0.985606$.

* Outage of line 11 ignored. 
Table 5.26: Effect of load variation on the frequency of different states - IEEERTS, considering total constraint set

\begin{tabular}{rrrrrr}
\hline $\begin{array}{l}\text { Load } \\
\text { Step }\end{array}$ & \multicolumn{1}{c}{$\begin{array}{c}\text { Normal } \\
\end{array}$} & Alert & $\begin{array}{r}\text { Frequency of } \\
\text { Emergency }\end{array}$ & $\begin{array}{c}\text { Ext. } \\
\text { Emergency }\end{array}$ & $\begin{array}{c}\text { No } \\
\text { Problem }\end{array}$ \\
\hline & & & & & \\
\hline $50 \%$ & 429.225654 & 5.754674 & 0.008594 & 0.005229 & 37.805541 \\
$50 \%$ & 428.484339 & 6.495549 & 0.009800 & 0.005229 & 37.804775 \\
$60 \%$ & 0.000000 & 434.762176 & 0.407950 & 0.005779 & 37.623786 \\
$70 \%$ & 0.000000 & 434.452258 & 0.995694 & 0.006405 & 37.345333 \\
$80 \%$ & 0.000000 & 434.088383 & 0.957585 & 0.974042 & 36.779681 \\
$90 \%$ & 0.000000 & 431.227304 & 0.958156 & 7.893171 & 32.721060 \\
$100 \%$ & 0.000000 & 396.685555 & 0.850784 & 54.855049 & 20.408278 \\
annual & 112.641546 & 321.100773 & 0.597472 & 1.763745 & 36.696155 \\
\hline
\end{tabular}

* Outage of line 11 ignored.

in order to operate a power system with a high normal state probability for a particular load level.

\subsection{SORTING}

The effect on the overall computation time of sorting is presented in Table 5.29 for the two test systems. In the case of the IEEE-RTS system, the total saving in CPU time is quite significant. The CPU time with line and generator contingency sorting is less than $50 \%$ of that without sorting.

\subsection{SUMMARY}

The assessment of the quality of power supplied to the load buses is considered in this thesis in terms of the voltage level at these load points. A good quality of service at a load bus implies that the voltage level at the bus does not deviate beyond permissible limits. Certain outages, mostly line outages, may result in voltage deviations beyond the limits at some of the load buses. A quantitative treatment of these events can be easily 
Table 5.27: Effect of voltage setting on the probability of different states - IEEERTS, considering total constraint set

\begin{tabular}{rccccc}
\hline $\begin{array}{l}\text { Load } \\
\text { Step }\end{array}$ & Normal & Alert & $\begin{array}{c}\text { Probability of } \\
\text { Emergency }\end{array}$ & $\begin{array}{c}\text { Ext. } \\
\text { Emergency }\end{array}$ & $\begin{array}{c}\text { No } \\
\text { Problem }\end{array}$ \\
& & & & & \\
\hline & & & & & \\
\hline $50 \%$ & 0.937659 & 0.006980 & 0.000009 & 0.000003 & 0.040955 \\
$60 \%$ & 0.935633 & 0.009006 & 0.000010 & 0.000003 & 0.040954 \\
$70 \%$ & 0.935052 & 0.009585 & 0.000014 & 0.000004 & 0.040952 \\
$80 \%$ & 0.932977 & 0.011663 & 0.000012 & 0.000003 & 0.040952 \\
$90 \%$ & 0.868487 & 0.070789 & 0.000011 & 0.011279 & 0.035040 \\
$100 \%$ & 0.549567 & 0.328664 & 0.000008 & 0.086145 & 0.021222 \\
annual & 0.920811 & 0.022297 & 0.000012 & 0.002553 & 0.039934 \\
\hline
\end{tabular}

Total probability considered in all cases $=0.985606$.

* Outage of line 11 ignored.

done by simply treating them as system problems without taking any further corrective action. Such a treatment, however, cannot recognize the actual severity associated with the outage event and quantitative indices cannot be produced for different operating states. A LP model for voltage correction and Q-load curtailment has been developed and is presented in this chapter.

The probabilistic indices for different operating states have been calculated considering voltage problems. A basic electric power utility objective should be to operate in the normal state with a high probability. A system may be capable of operating within constraints but may not be able to withstand outages, even single level events at some initial voltage settings. Annualized indices and annual indices are presented in this chapter recognizing the voltage problem as well as for the total constraint set. A reduction in the computation time for the solution of generator contingencies and both generator and line contingencies can be obtained by sorting the identical contingencies. This approach can result in a significant saving in the computation time for large systems. 
Table 5.28: Effect of voltage setting on the frequency of different states - IEEERTS, considering total constraint set

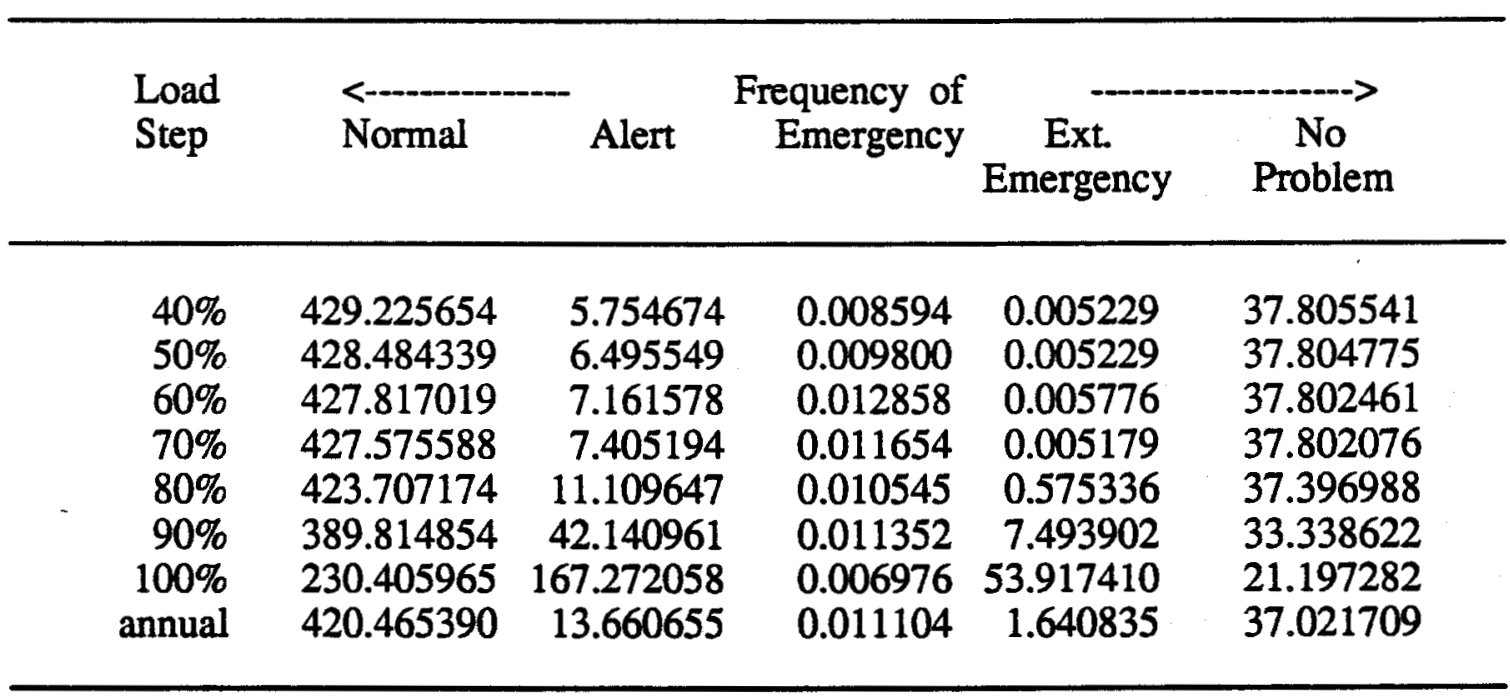

* Outage of line 11 ignored.

Table 5.29: Comparison of contingency considered and computation time with and without sorting

Sorting Contingency CPU Time

For MRBTS

No

Generator

Generator + Line
1489

566

566
1.99 Seconds

1.08 Seconds

1.08 Seconds

For IEEE-RTS

No

Generator

Generator + Line
91560

24604

19967
14 Min. 5.45 Sec.

7 Min. 39.43 Sec.

6 Min. 19.41 Sec. 
The probabilistic indices for different operating states displayed up to this point in this thesis have been calculated using the enumeration technique. It is also possible to utilize the simulation method together with the enumeration approach in a hybrid form to calculate these indices. The Monte Carlo simulation method can be used to determine the system states and then by using the enumeration technique, the sampled states can be assigned to an appropriate system operating state. The total sampled probability or a fraction of the sampled probability can be assigned to different operating states. Two hybrid approaches are presented for calculating the indices associated with different operating states in the next chapter. 


\section{A HYBRID MODEL FOR RELIABILITY EVALUATION OF COMPOSITE POWER SYSTEMS}

\subsection{INTRODUCTION}

The two most popular approaches in the computation of composite system reliability indices are the contingency enumeration method and the Monte Carlo simulation technique. The state or contingency enumeration approach [71] includes the systematic selection and evaluation of contingencies, the classification of each contingency according to specified failure criteria and the accumulation of reliability indices. In order to compute annual composite system reliability indices, it is necessary to repeat the calculations for several conditions representing different seasons, major maintenance periods, periods with different transfer conditions and different load levels, etc.. Computation time requirements tend to limit the number of system conditions for large scale systems. The use of the Monte Carlo simulation method allows detailed modelling of precontingency conditions, generation and transmission outages and operating practices $[17,71]$. Parameters that define a system operating state, such as load and the state of generation and transmission components, are selected by random sampling in accordance with the probability distributions of the parameters. The selected operating state is tested and the outcomes evaluated according to one or more failure criteria. A key feature of the Monte Carlo simulation method is the ability to sample events obeying any probability distribution.

In this chapter, the indices for different system operating states have been calculated using two hybrid approaches. The system situation is selected by random sampling and the sampled situation is assigned using the enumeration approach to an appropriate system operating state. Two hybrid approaches have been investigated and are reported in this chapter. 
The system situation i.e. load, generators on forced outage, transmission lines on forced outage, etc. can be determined by random sampling. Random number generator, therefore, is an essential element of a basic Monte Carlo simulation method. A brief description of random number generation is given in Appendix C.

\subsection{SIMULATION METHODS FOR POWER SYSTEM RELIABILITY EVALUATION}

A simulation $[103,104]$ is an imitation of the operation of a system over a period of time. It involves the generation of an artificial history of a model for the system and the observation of that artificial history to draw inferences concerning the operating characteristics of the real system. This method requires a large amount of computing time and storage to develop a good model for the system. It is not, therefore, used extensively if alternate analytical methods are available. The simulation technique, however, is easy to apply and can include system effects which may have to be approximated in an analytical method.

There are two basically different simulation approaches available for power system reliability evaluation, namely

1. sequential state transition sampling and

2. system state sampling.

The state transition sampling approach is based on sampling the probability distribution of the state duration. System state sampling is based on sampling the state probability. The former requires a large amount of computer time and storage to create an artificial chronological component state transition process. The latter directly samples the system state and is fast and is therefore used in this research work. The main steps in the system state sampling method are:

\section{1. $i=0$}

2. set $i=i+1$ for the next component

3. select a random number $X_{i}$ distributed uniformly between $[0,1]$ using a random number generator

4. the state of the $\mathrm{i}^{\text {th }}$ component 


$$
S_{i}= \begin{cases}1 & \text { normal state if } X_{i}>F \\ 0 & \text { outage state if } 0 \leq X_{i} \leq \text { FOR }_{i}\end{cases}
$$

5. if $\mathrm{i}<\mathrm{n}$, number of component, go to step 2 otherwise step 6

6. system situation, containing $\mathrm{n}$ component, is expressed by the vector $S=\left(S_{1}, S_{2}, S_{3}, \ldots \ldots . ., S_{n}\right)$

After determining the system state, an appropriate method can be used to analytically solve the sampled situation. The hybrid models discussed in the next section are utilized to determine the different state probabilities from the sampled situation determined above.

\subsection{HYBRID MODELS}

After determining the system situation by random sampling, the indices can be calculated by assigning the total sampled probability or a fraction of the sampled probability to different operating states. A brief description of the two hybrid methods investigated in this research work is given below.

\subsubsection{Method 1}

In this method, the system situation is determined by random sampling as noted earlier. Let the system situation be denoted by the vector $S_{1}$. The system situation $S_{1}$ is examined by the approximate selection method and if $S_{1}$ does not create a system problem, then one more level outages are considered with the present sampled situation using the enumeration method. Consider that the sampled situation $S_{1}$ represents generator 1 on an outage condition and all other components in the operating state and that this system situation does not create any system problem. The situation with generator 1 on outage, considered with all other component single level outages is shown in Table 6.1. The fraction of the probability of the sampled outage of generator 1 is calculated using the relation

$$
\mathrm{P}_{1}+\mathrm{P}_{1} \mathrm{P}_{\mathrm{g} 2}+\mathrm{P}_{1} \mathrm{P}_{\mathrm{g} 3}+\ldots+\mathrm{P}_{1} \mathrm{P}_{11}+\mathrm{P}_{1} \mathrm{P}_{12}+\ldots=1 / \mathrm{Numb}
$$

or,

$$
P_{1}=\left[1.0 /\left(1.0+P_{g 2}+P_{g 3}+\ldots+P_{11}+P_{12}+\ldots\right)\right]^{*}[1 / \text { Numb }]
$$


where:

$\mathrm{Numb}=$ number of samples considered in the study and

$\underbrace{}_{\mathrm{g} 1}=($ Probability of all component in $) *\left(\lambda_{\mathrm{g} 1} / \mu_{\mathrm{g} 1}\right)$ and so on.

Table 6.1: Outages considered using enumeration with generator 1 outage by sampling

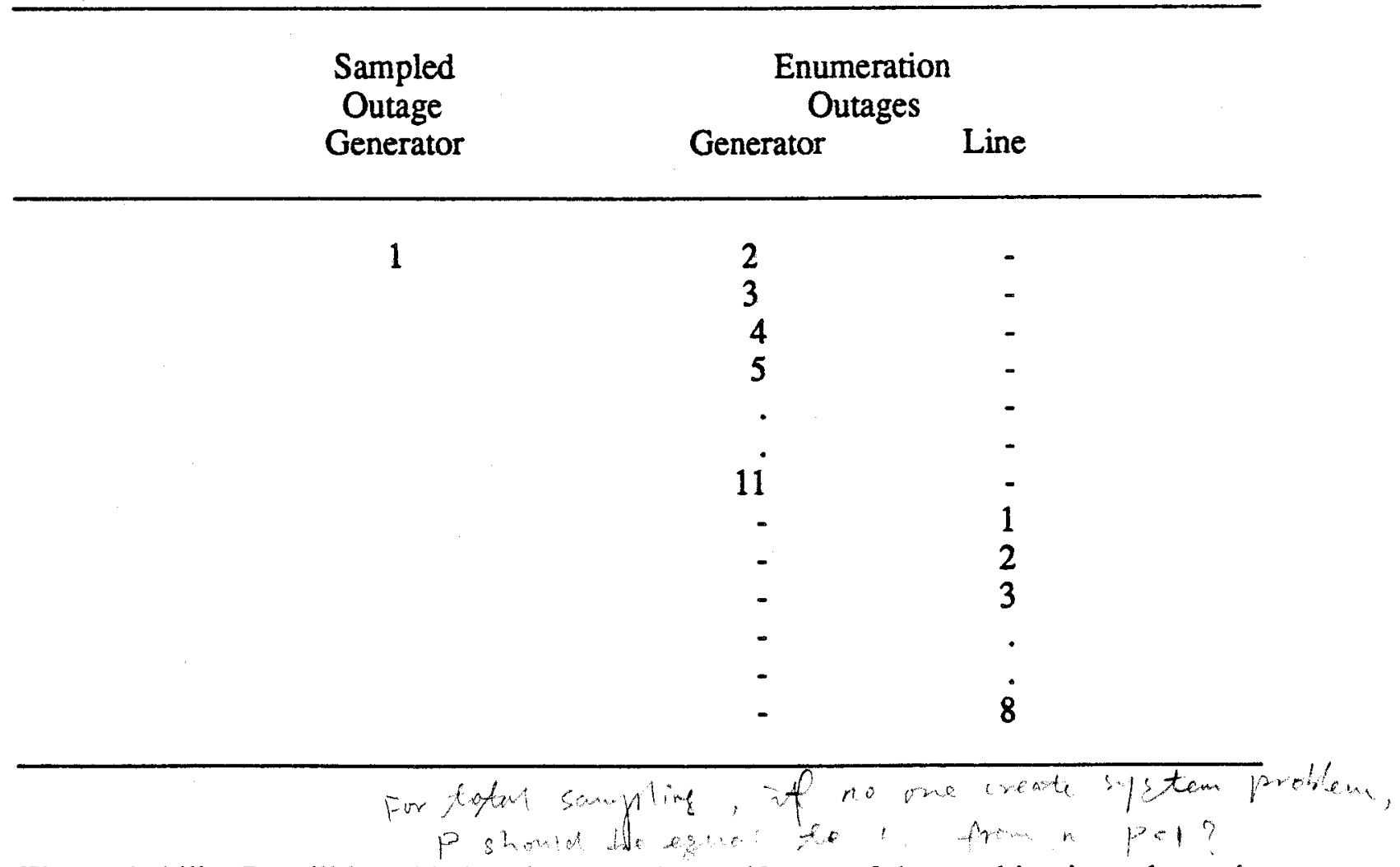

The probability $P_{1}$ will be added to the normal state if none of the combinations shown in Table 6.1 create a system problem, otherwise $P_{1}$ will be added to the alert state. The combinational probabilities will go to other system states depending upon the system problem and the corrective actions. For example, if the generator 1 and line 1 combined outage creates a system problem and no load curtailment is required to alleviate the problem, then the probability $P_{1}{ }^{*} P_{11}$ will be added to the emergency state. If load curtailment is required to alleviate the system problem then this probability will be added to the extreme emergency state. If the above combination does not create any system problem, then this probability, $\mathrm{P}_{1}{ }^{*} \mathrm{P}_{11}$ will be added to the no problem state since a decision cannot be taken on whether it belongs to the normal or the alert state. On the 
other hand, if $S_{1}$ itself creates a system problem then the fraction of the probability, $P_{1}$, of occurrence of this system situation is added to the probability of the emergency or the extreme emergency state depending upon the amount of load curtailment. Then one more level outages are considered with the $S_{1}$ sampling situation using the enumeration method as described earlier.

In the case of an extreme sampling situation i.e. for the following sampling conditions

1. three lines out,

2. two generators and one line out,

3. one generator and two lines out or

4. four generators out

no additional outages are considered. The full sampling probability $(=1 / \mathrm{Numb})$ of this sampling situation is added to the appropriate system state after checking this sampling status. A brief flow chart for Method 1 is shown in Figure 6.1.

\subsubsection{Discussion Of The Results - Method 1}

The results obtained using this method are shown in Figures 6.2 to 6.6 and Figures 6.7 through 6.11 for the MRBTS and IEEE-RTS respectively. It can be seen from these figures that the results converge to a value which is quite different from the actual analytical value. The probability of the extreme emergency state, for example, is more than double that of the analytical value in the simulation case for the MRBTS. The reason for this is that for those sample situations which require load curtailment, the probabilities are being assigned directly to this state and some of the probabilities from those sample situations which do not create a system problem but with enumeration combination outages do create a system problem and require load curtailment are also being added to this state. The probability and frequency of the extreme emergency state for the IEEE-RTS is also very large compared to the analytical value. In this case, the probability and frequency of the emergency state are more than double that of the analytical value. The reason could be that for this system some of the sampled situations which create system problems and require no load curtailment as well as many of the 


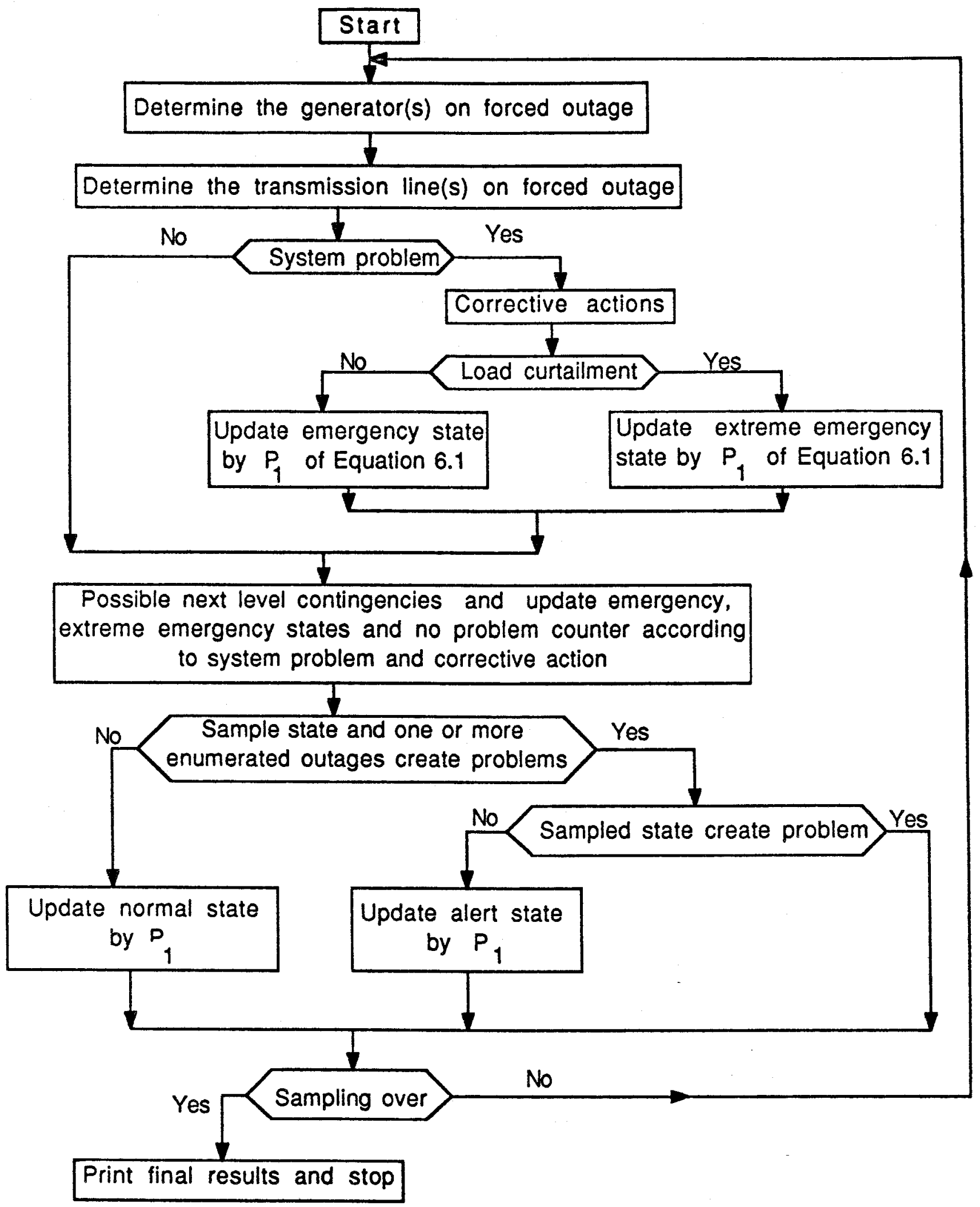

Figure 6.1: Flow chart for hybrid Method 1 
enumerated outages which do create system problem and do not require load curtailment are added to this system state and therefore inflate the final values. In Figure 6.7, the probability and frequency of the normal state drops from a relatively high value to zero on the 1256th sample, because for this sample, the sample situation is a single level outage (line 27) which creates a voltage problem. Therefore, according to the definition of the normal state, this system has no normal state since it cannot withstand all the first level outages and the probability and frequency reduces to zero. The jump in the probability and frequency of the alert state as seen in Figure 6.8 can be explained in a similar way. The indices obtained by this method are not considered to be satisfactory as compared to the results obtained using the analytical approach. In order to avoid the difficulty noted above, the following method (Method 2) was utilized to select and analyze the different system states using both simulation and enumeration.

\subsubsection{Method 2}

In this approach, the sampling situation is determined using the random sampling procedure described earlier. If the sampled situation creates a system problem, the probability and frequency of the emergency or the extreme emergency state are enhanced depending upon the amount of load curtailment. If the sampled situation $S_{i}$ does not create a system problem, then the enumeration method is utilized to determine whether the sampling situation belongs to the normal or to the alert state. As soon as any system problem is encountered with the enumeration outages, no more outages using the enumeration method are considered and the indices for the alert state are enhanced. If none of the enumerated states create any system problem, the indices for the normal state are enhanced. In all the cases instead of adding a fraction of the sampling situation probability, the full sampling probability $(=1.0 / \mathrm{Numb})$ is added to the appropriate system state. In the case of extreme sampling situations noted earlier, if $S_{i}$ does not create a system problem then the 'no problem' counter is enhanced since no further enumerated outages are considered with this sampling situation. A brief flow chart for this method is shown in Figure 6.12. The results obtained with this method are shown in Figures 6.13 to 6.22 for the two test systems. The number of contingencies for each outage level are shown in Table 6.2 and 6.3 for different numbers of samples. As can be seen from Figures 6.13 to 6.22 , the indices converge to the corresponding analytical values as the 

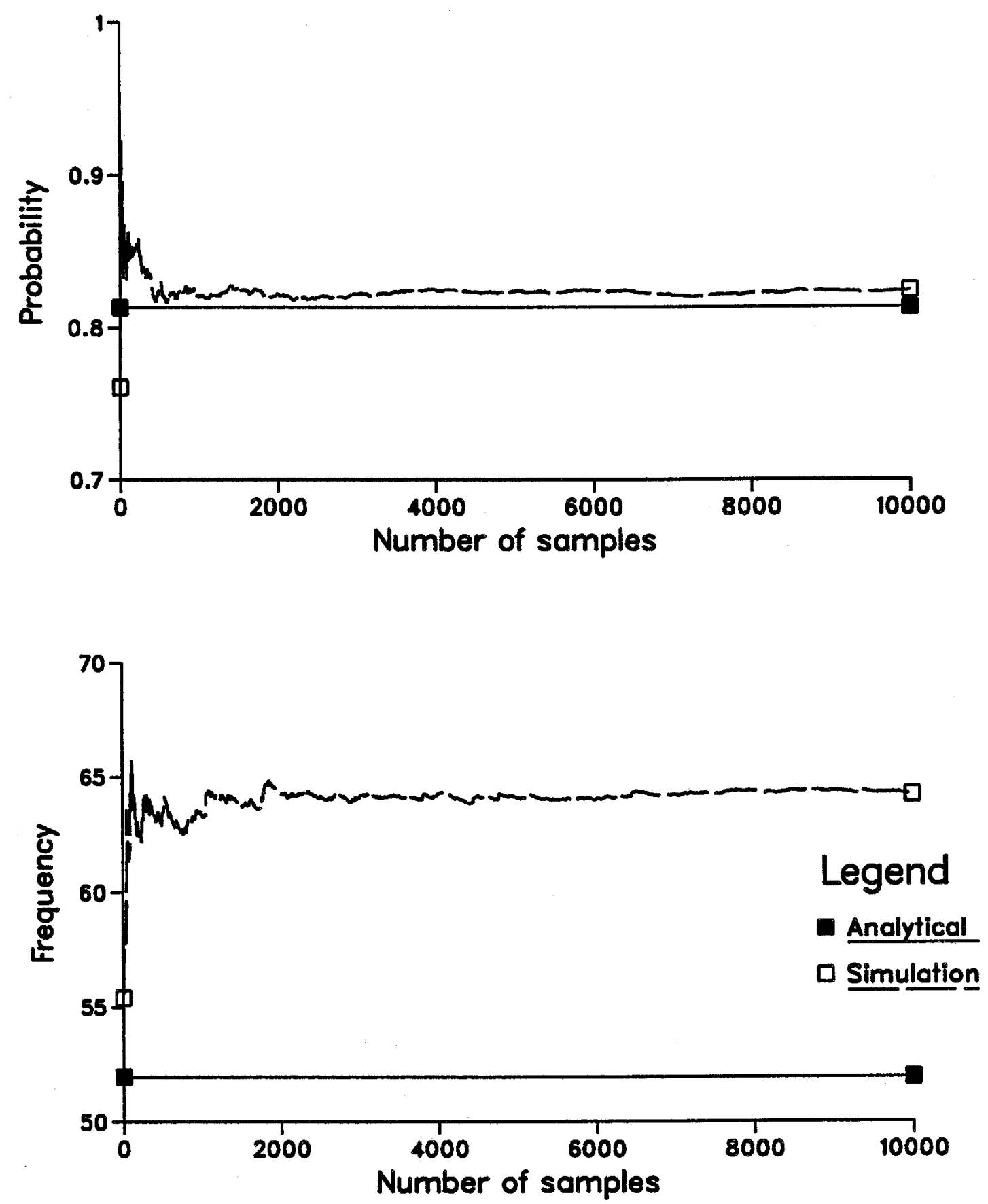

Figure 6.2: Probability and frequency of the normal state with number of samples using Method 1 - MRBTS 

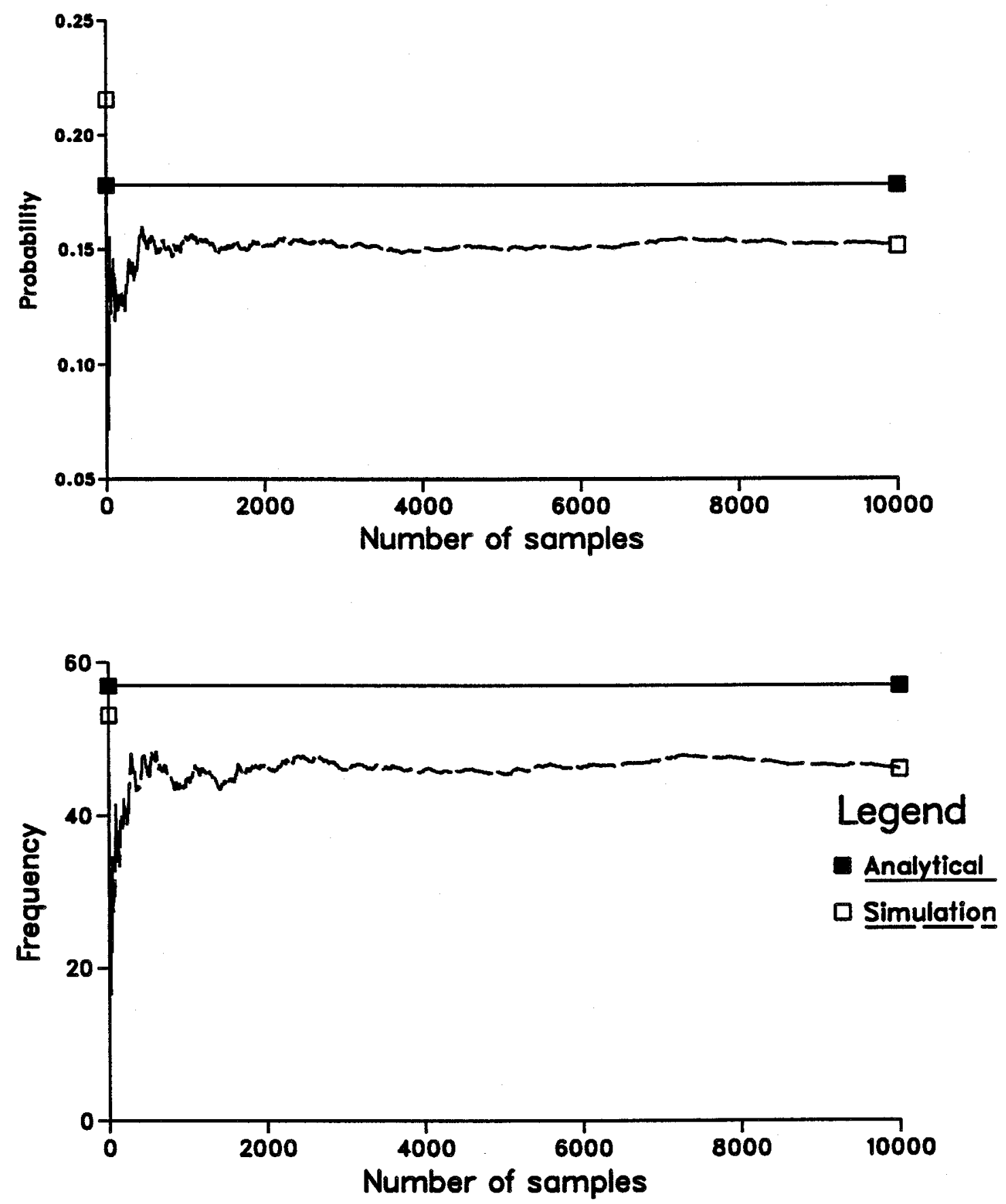

Figure 6.3: Probability and frequency of the alert state with number of samples using Method 1 - MRBTS 


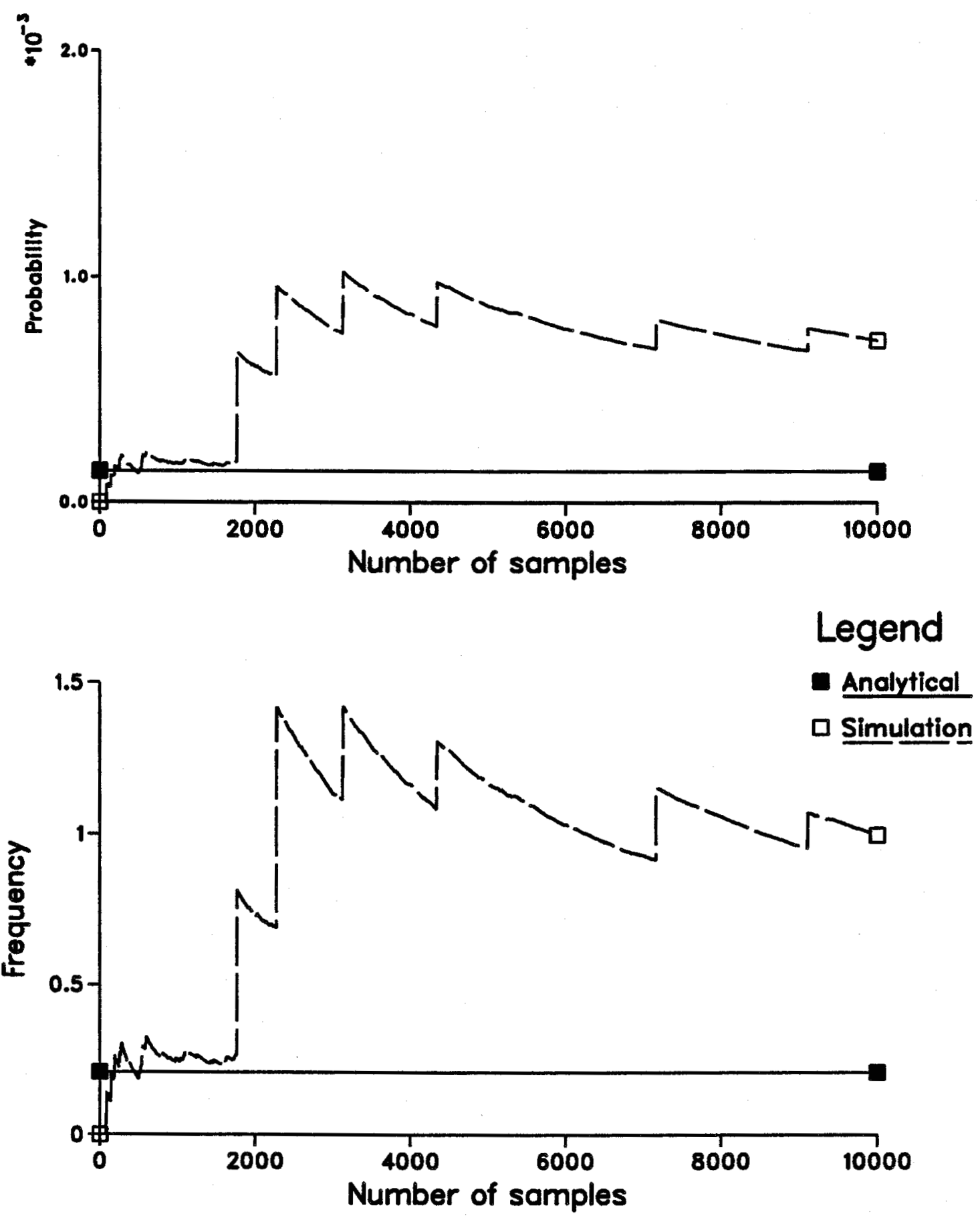

Figure 6.4: Probability and frequency of the emergency state with number of samples using Method 1 - MRBTS 

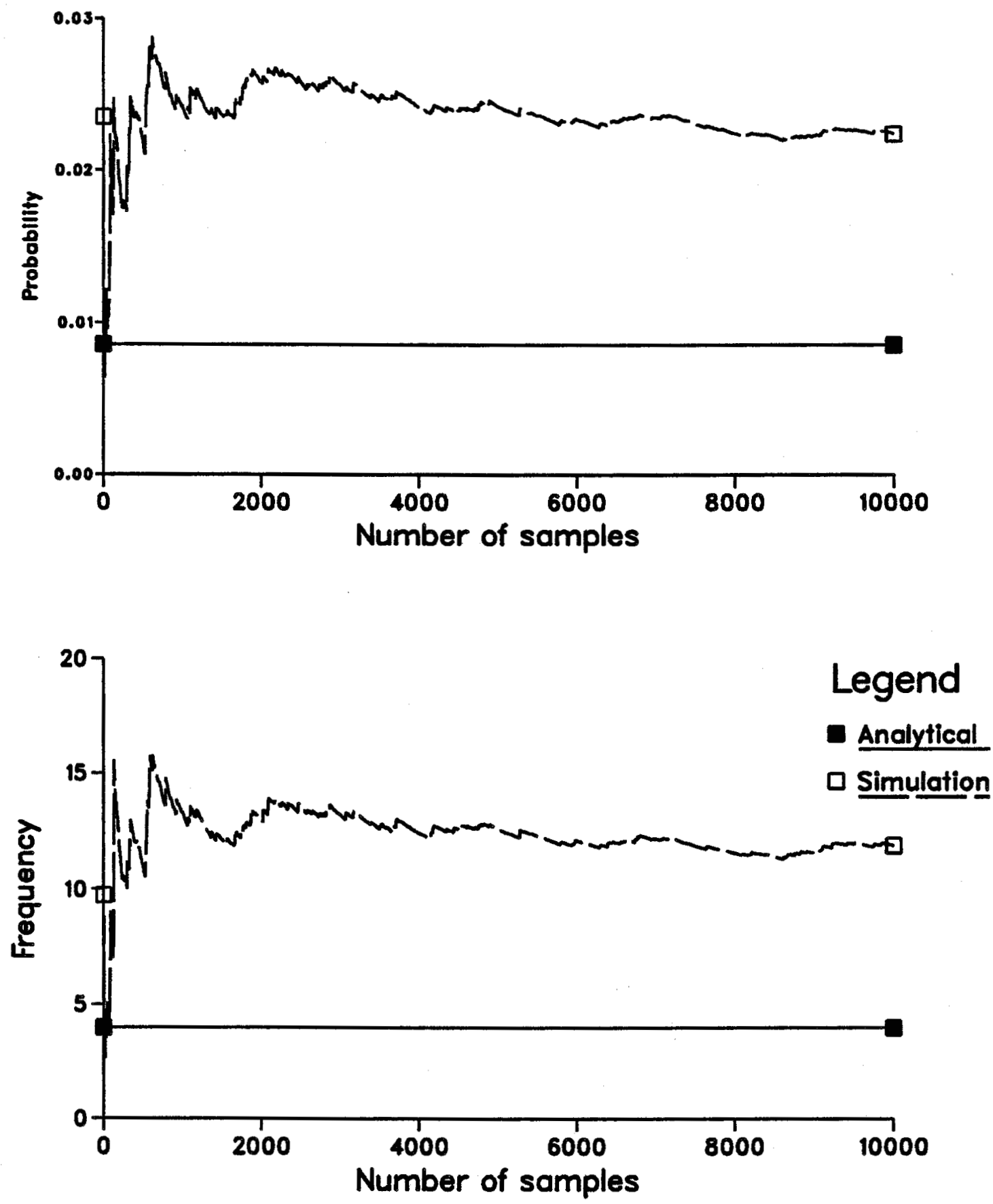

Figure 6.5: Probability and frequency of the extreme emergency state with number of samples using Method 1 - MRBTS 

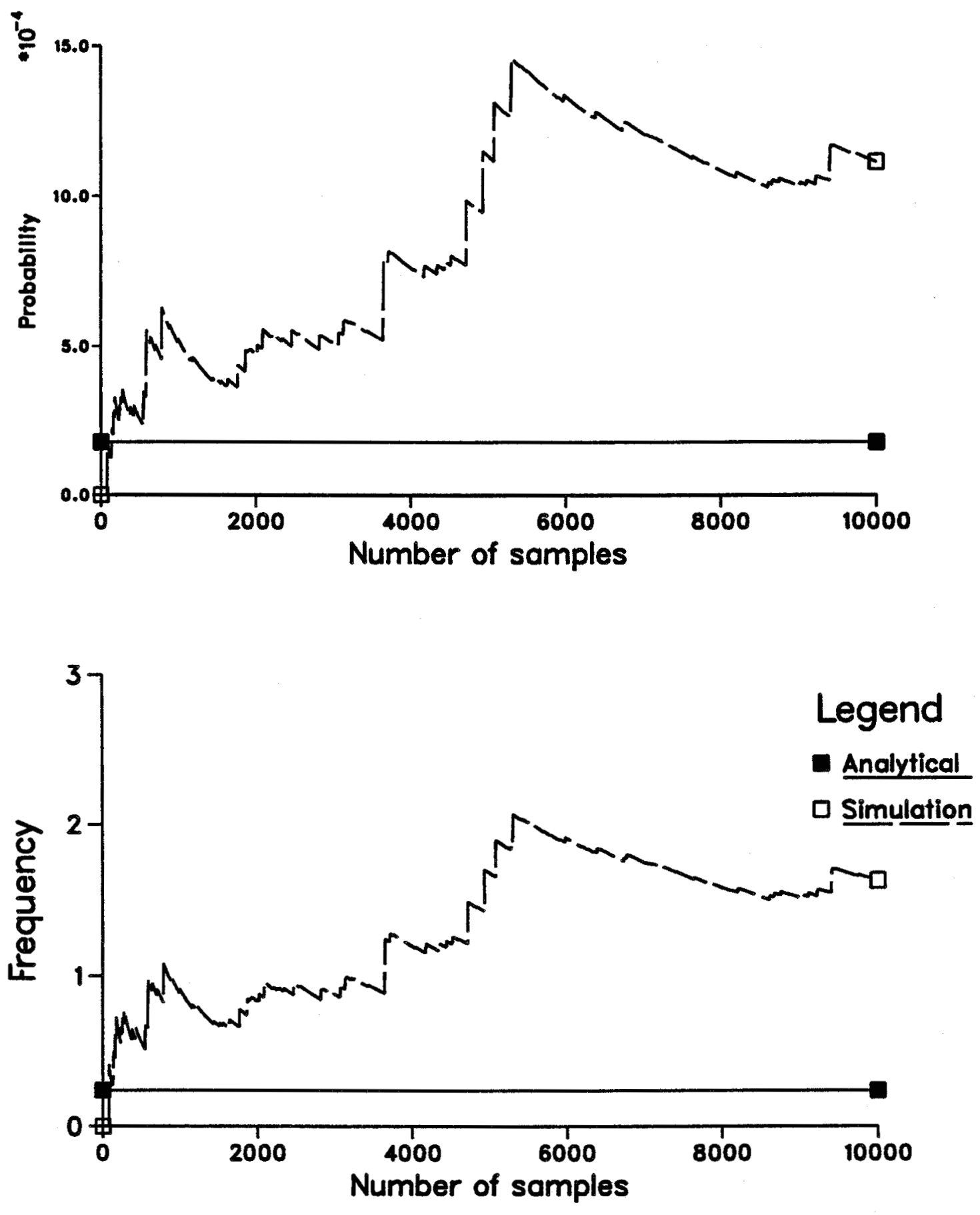

Figure 6.6: Probability and frequency of the no problem with number of samples using Method 1 - MRBTS 

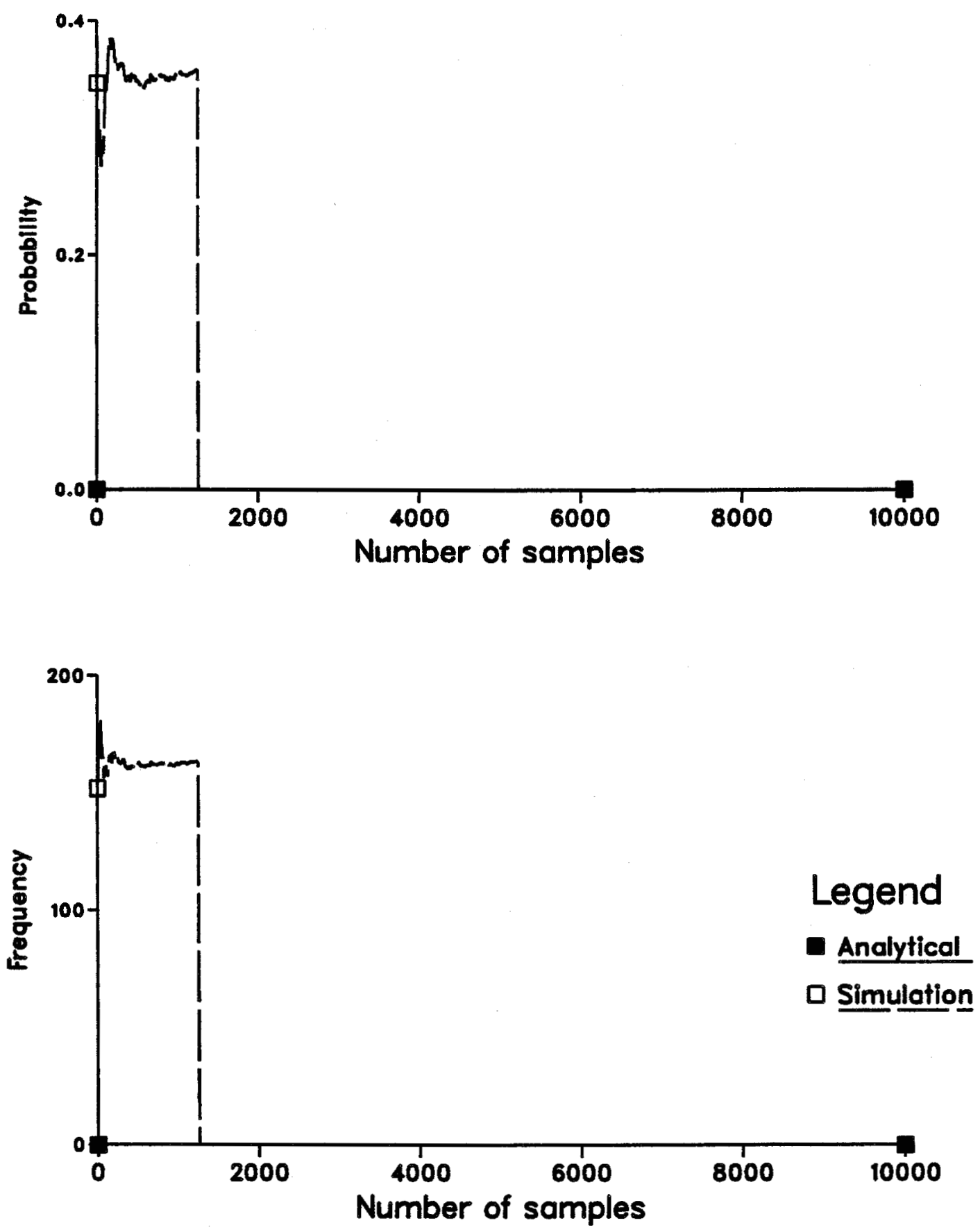

Figure 6.7: Probability and frequency of the normal state with number of samples using Method 1 - IEEE-RTS 

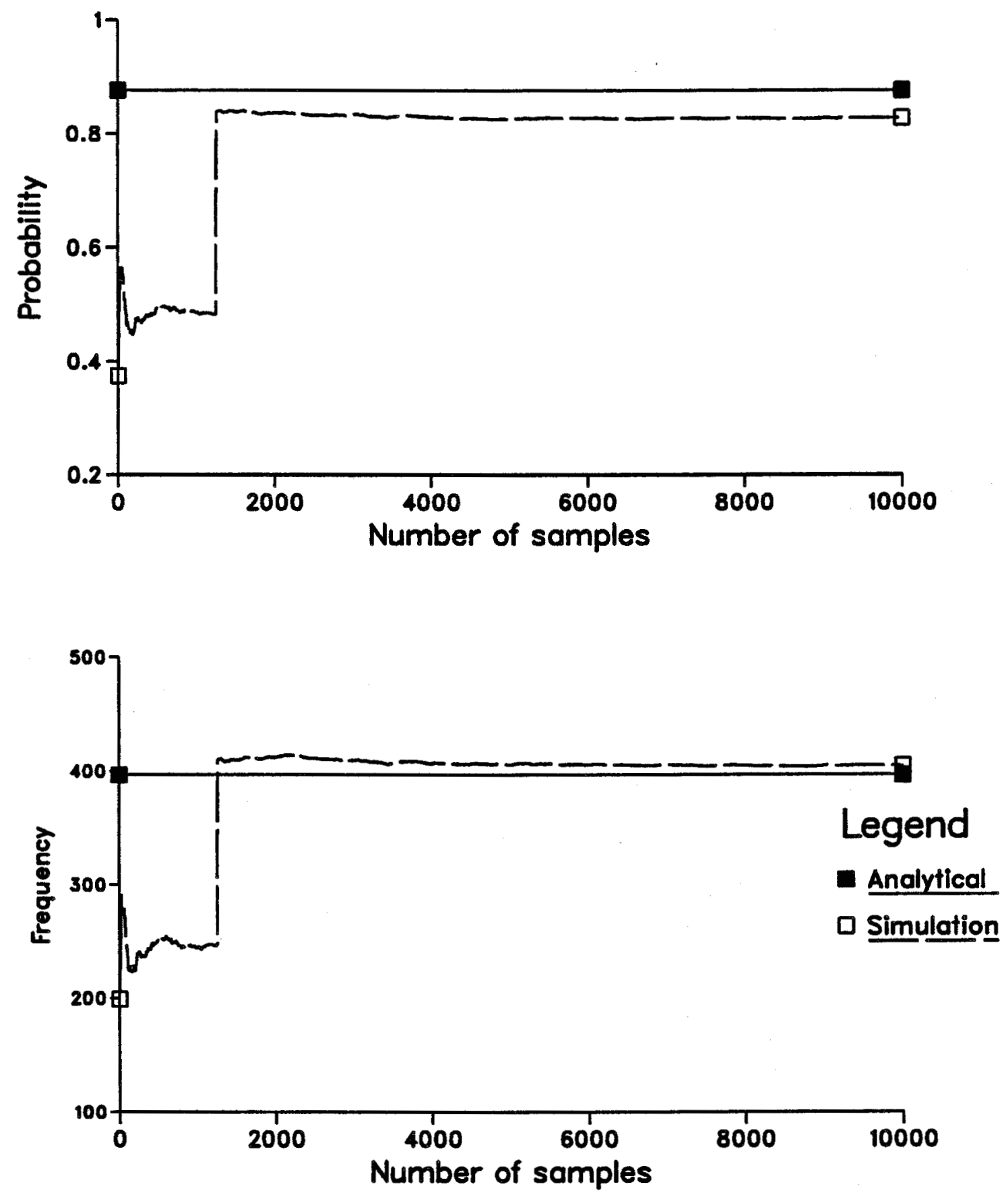

Figure 6.8: Probability and frequency of the alert state with number of samples using Method 1 - IEEE-RTS 

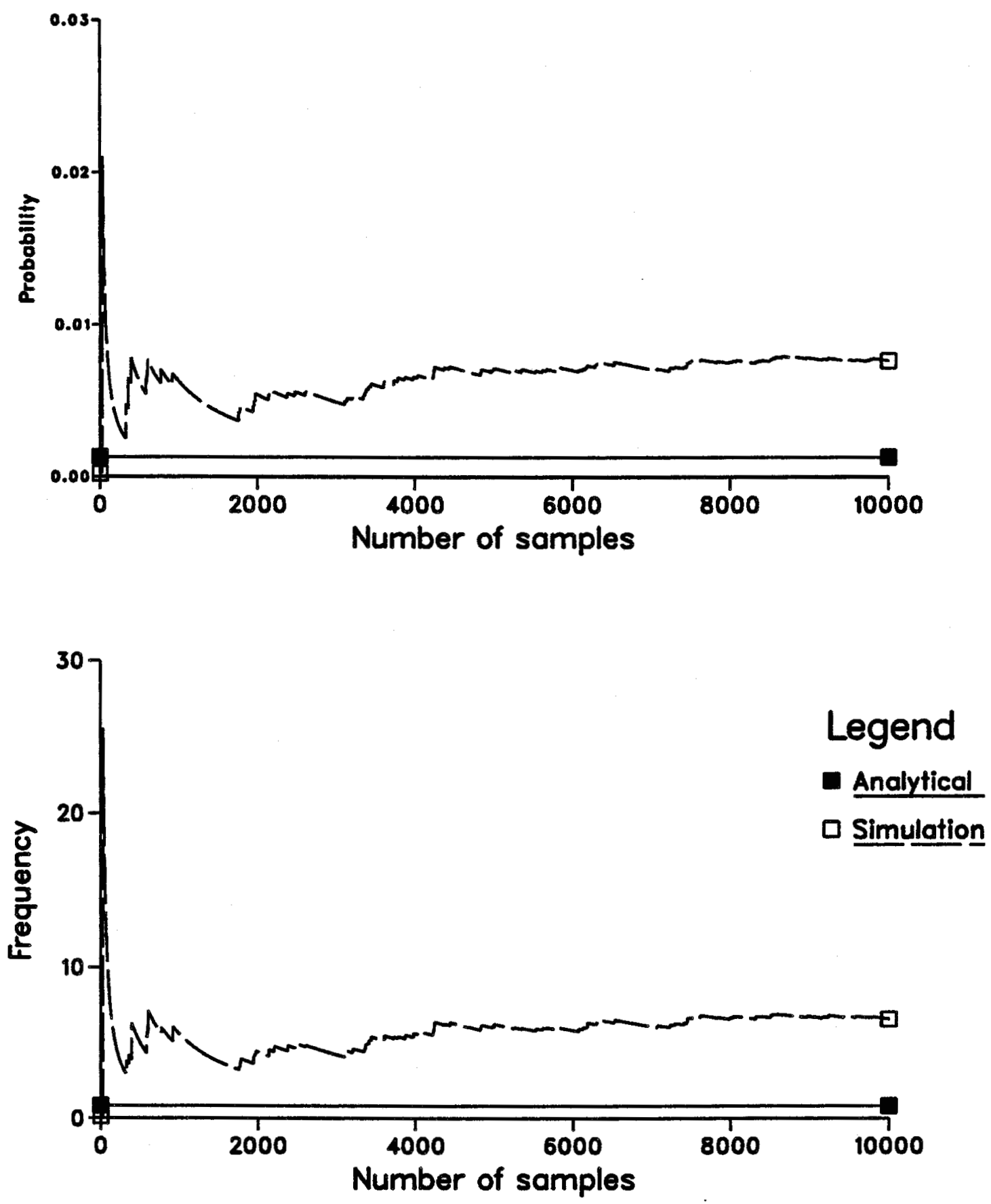

Figure 6.9: Probability and frequency of the emergency state with number of samples using Method 1 - IEEE-RTS 

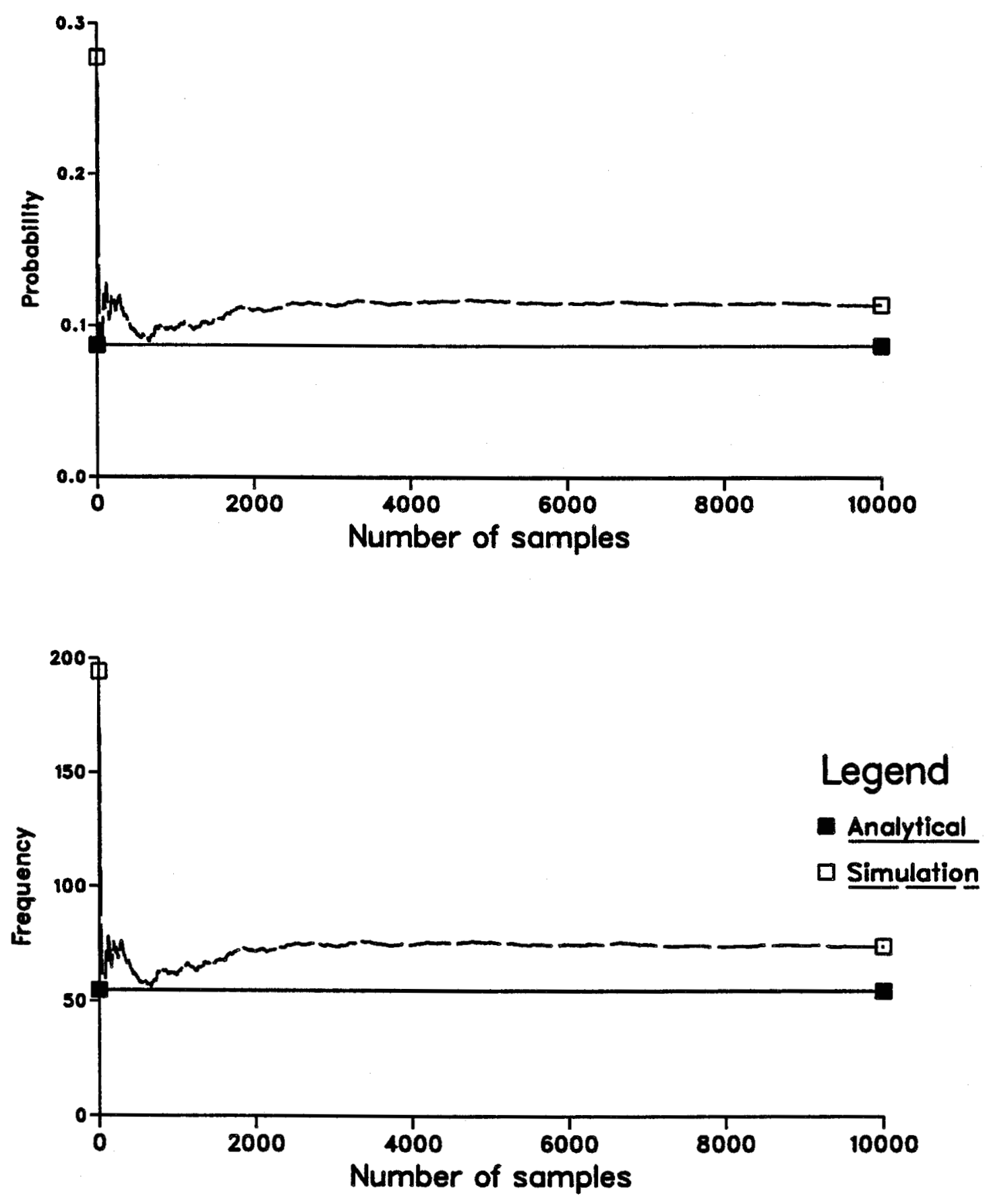

Figure 6.10: Probability and frequency of the extreme emergency state with number of samples using Method 1 - IEEE-RTS 

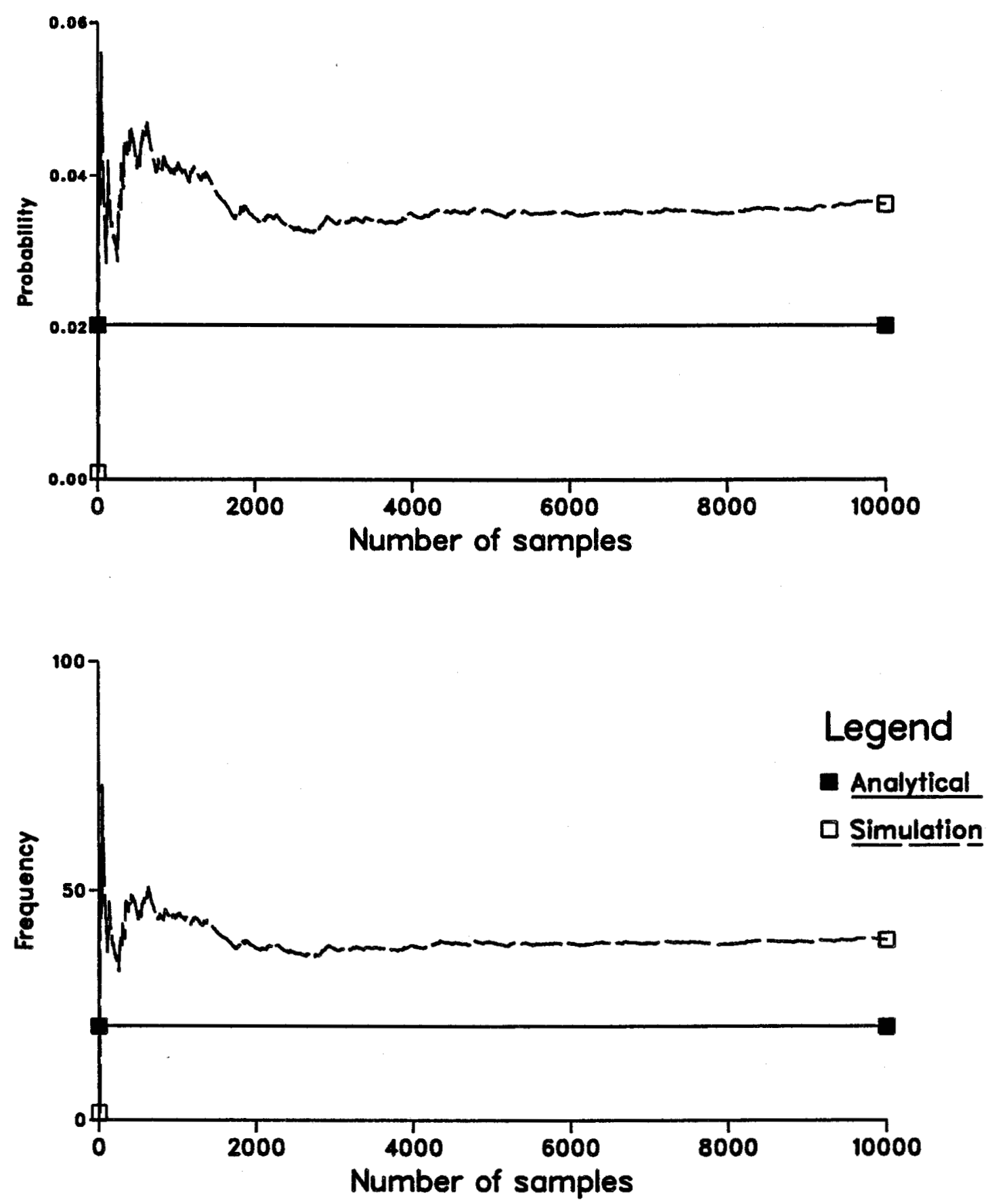

Figure 6.11: Probability and frequency of the no problem with number of samples using Method 1 - IEEE-RTS 


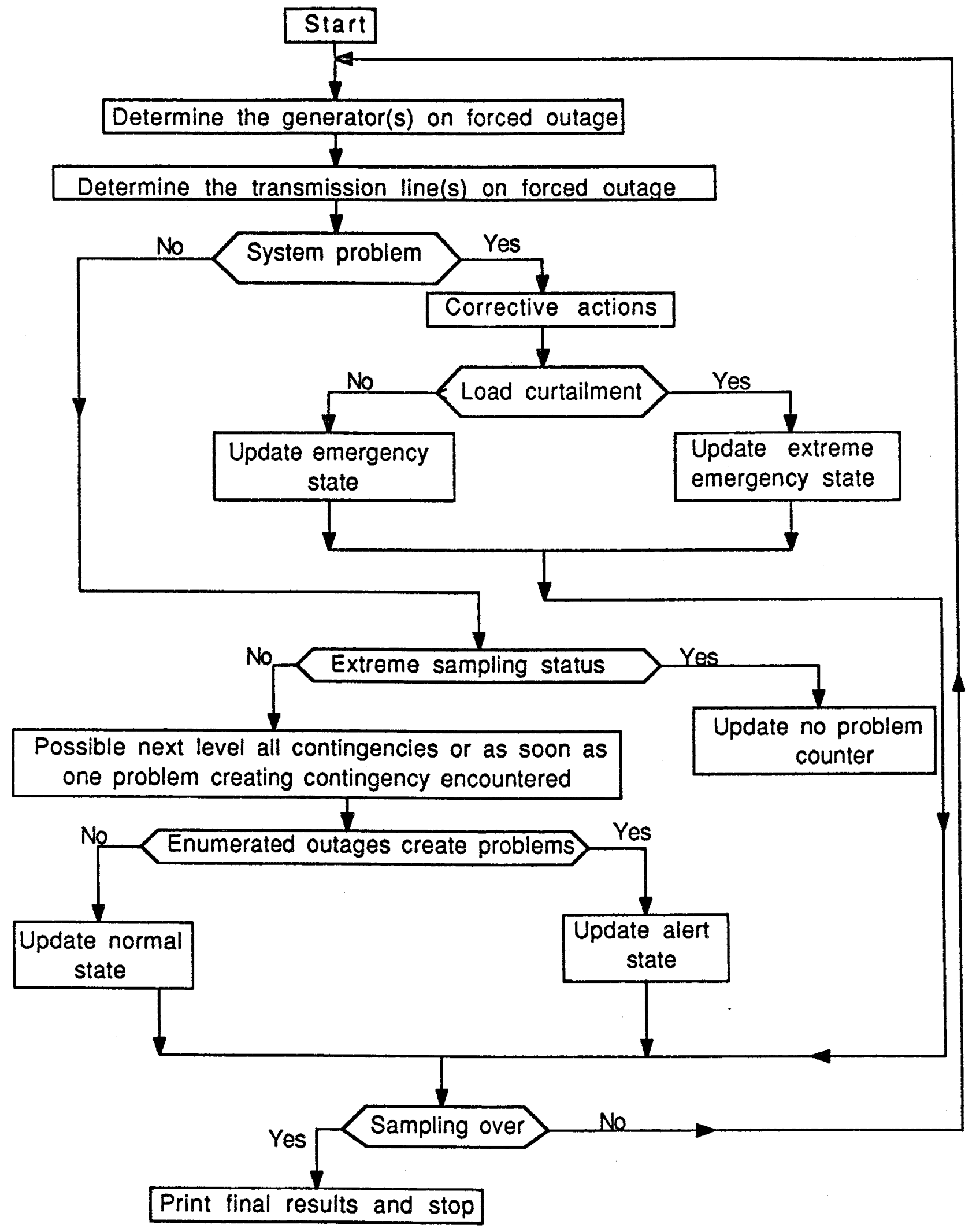

Figure 6.12: Flow chart for hybrid Method 2 
number of samples increases. The perturbations in the probabilities and frequencies of the normal and the alert states can be appreciated as stated in the discussion of Method 1. The probabilities and frequencies of the emergency state are zero for both the test systems for about 2000 samples since the sample situations up to this sampling number creates system problems which require load curtailment. Therefore, the probabilities and frequencies of the extreme emergency state for both the test systems have a value greater than zero at a very lower sampling number. This method provides results which are quite close to the analytical values and is therefore used throughout the remainder of the studies conducted in this chapter.

\subsection{STOPPING RULE}

The confidence interval for the expected results can be used as a stopping rule to terminate the simulation. The confidence interval for simulation is statistically based and can be expressed in terms of distribution free ratios of moments or for a large number of samples, expressed in terms of a confidence level using the " $t$ " distribution [69]. The ratio of the standard error of the estimate to the average value has been suggested in Reference [105]. The relative variance coefficient, $\alpha$, can be expressed as

$$
\alpha=\frac{S_{I}}{E(X)}
$$

where:

$$
\begin{aligned}
& \mathrm{X} \quad=\text { outcome of the sample system state } \\
& \mathrm{E}(\mathrm{X}) \quad=\text { expected value }=\frac{1}{N} \sum_{i=1}^{N} X_{i} \\
& \mathrm{~N} \quad=\text { number of trials or samples and } \\
& \mathrm{S}_{\mathrm{I}} \quad=\text { standard error of estimator }=\left[\frac{1}{N} \frac{1}{N-1} \sum_{i=1}^{N}\left[X_{i}-E(X)\right\}^{2}\right]^{0.5}
\end{aligned}
$$

The relative variance coefficient for the extreme emergency state has been utilized as a stopping rule. The convergence of the extreme emergency state is slower than the convergence of other states. On the other hand, only this state requires load curtailment whereas the other three states do not involve load curtailment. Therefore, the existance 

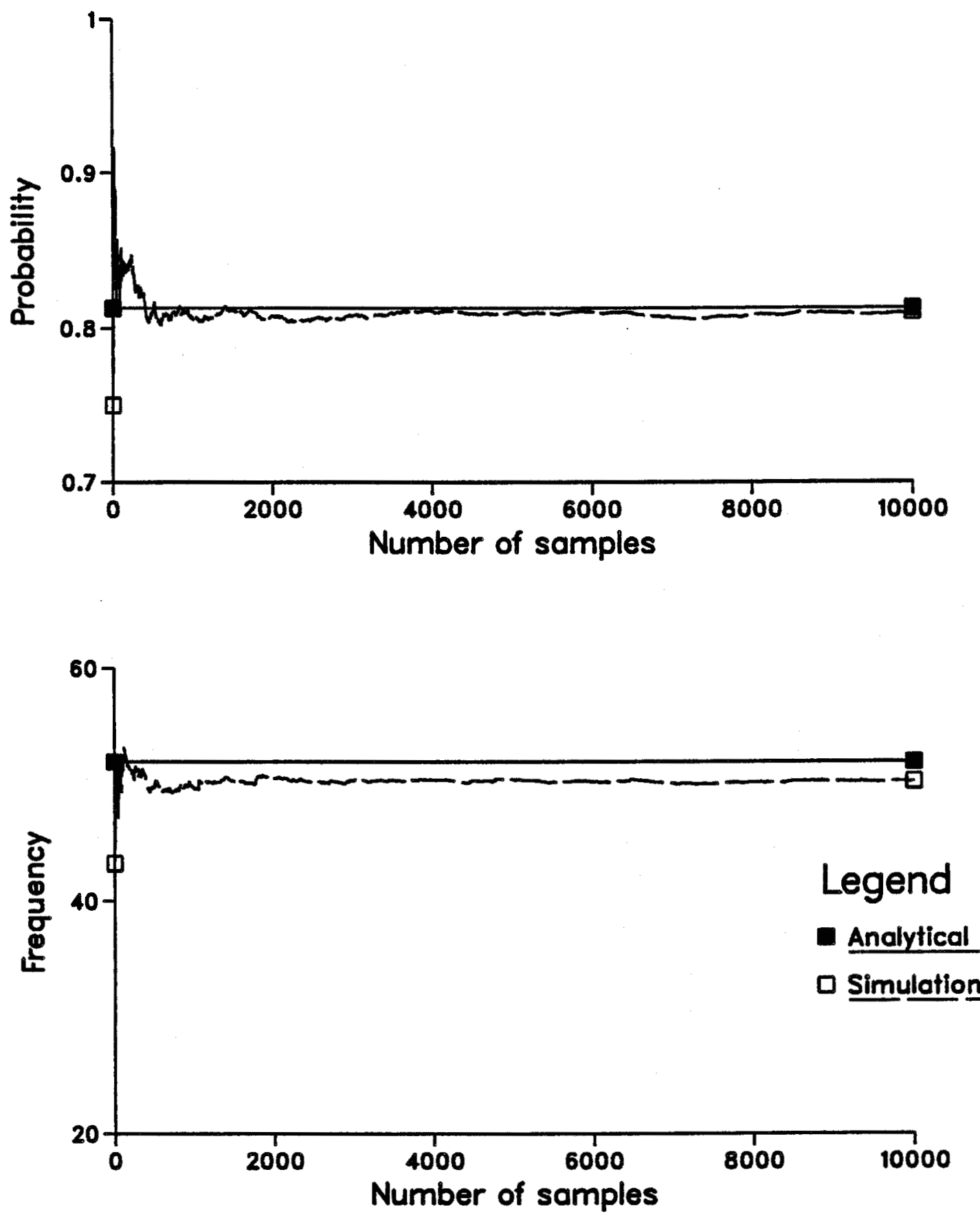

Figure 6.13: Probability and frequency of the normal state with number of samples using Method 2 - MRBTS 

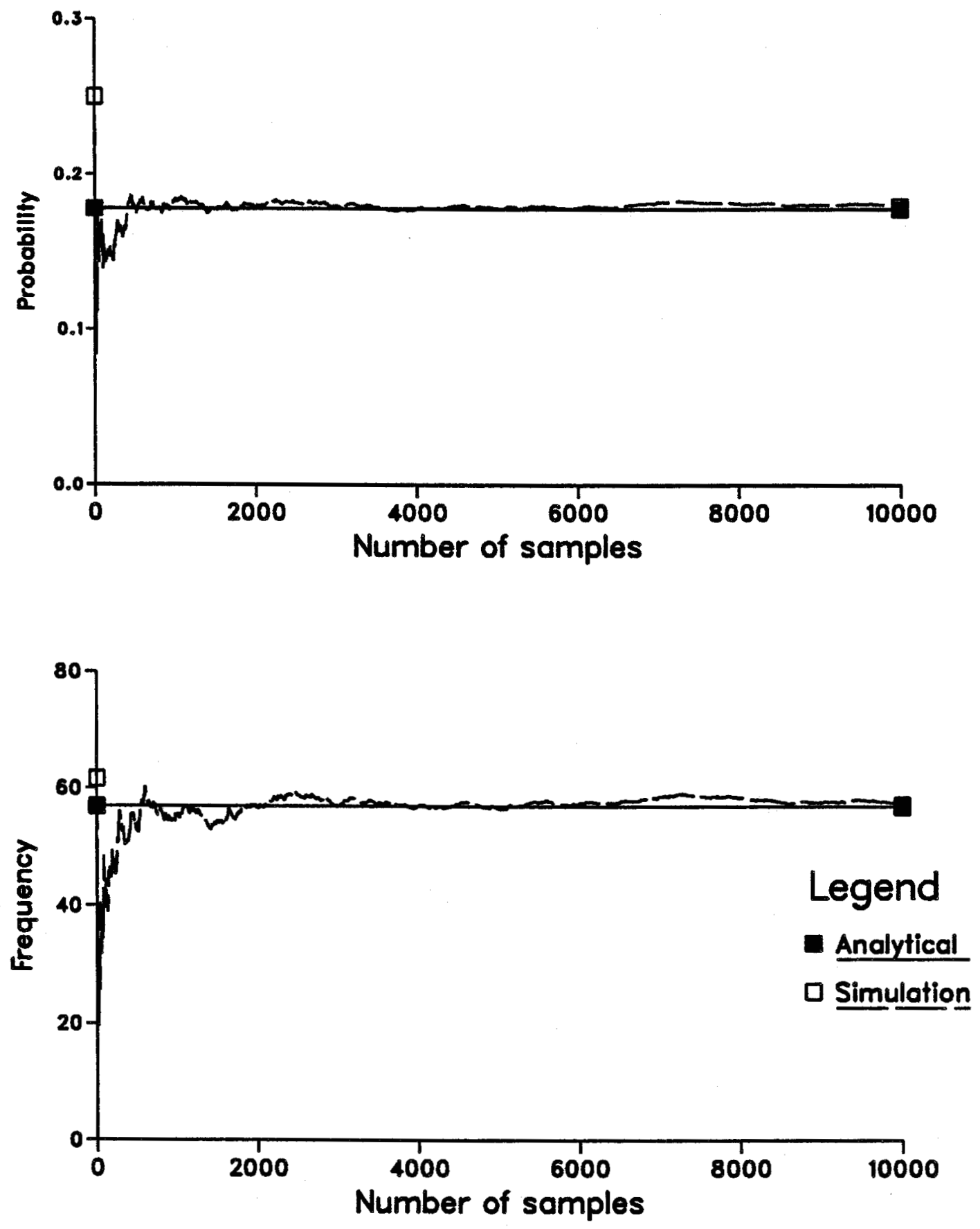

Figure 6.14: Probability and frequency of the alert state with number of samples using Method 2 - MRBTS 

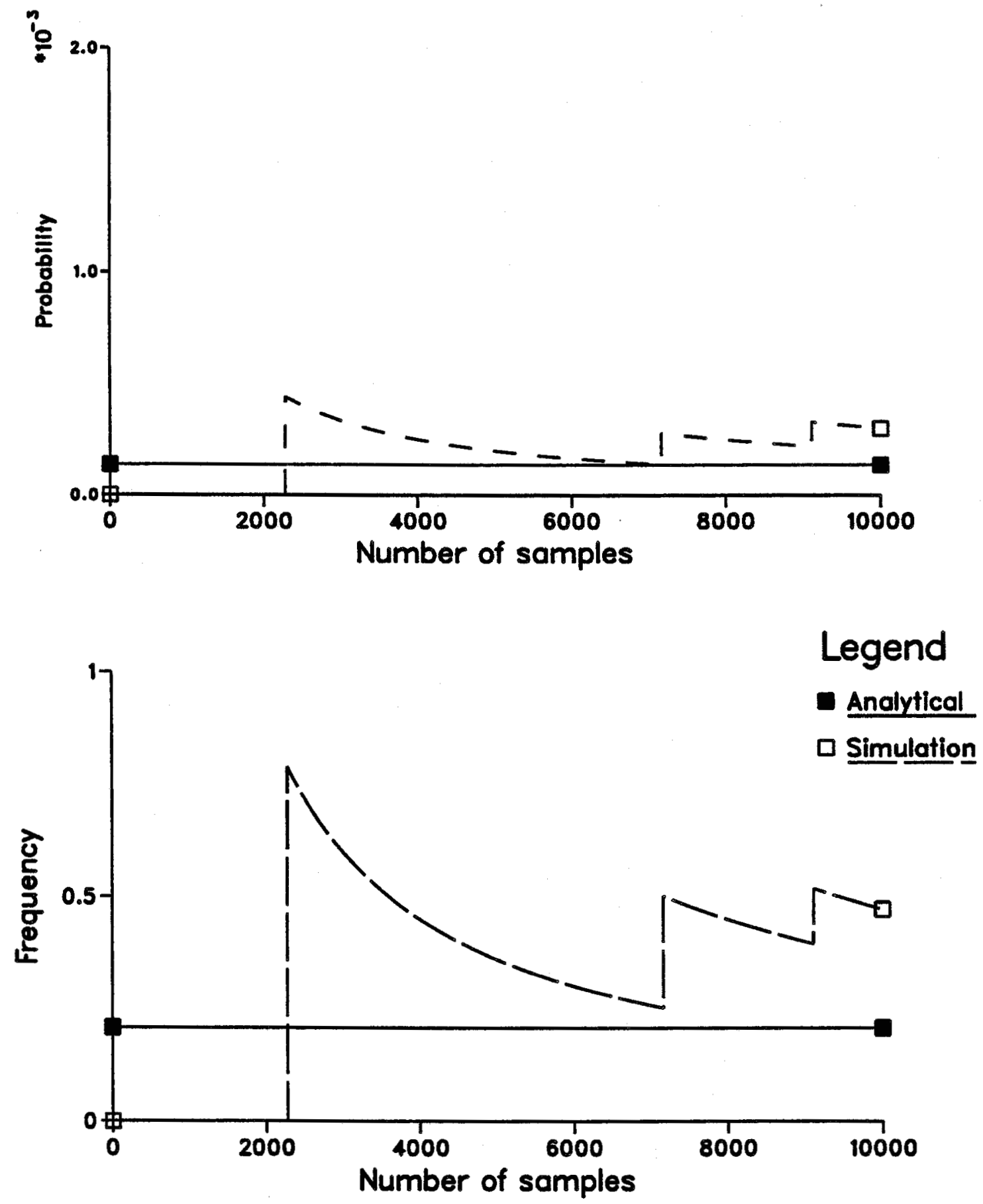

Figure 6.15: Probability and frequency of the emergency state with number of samples using Method 2 - MRBTS 

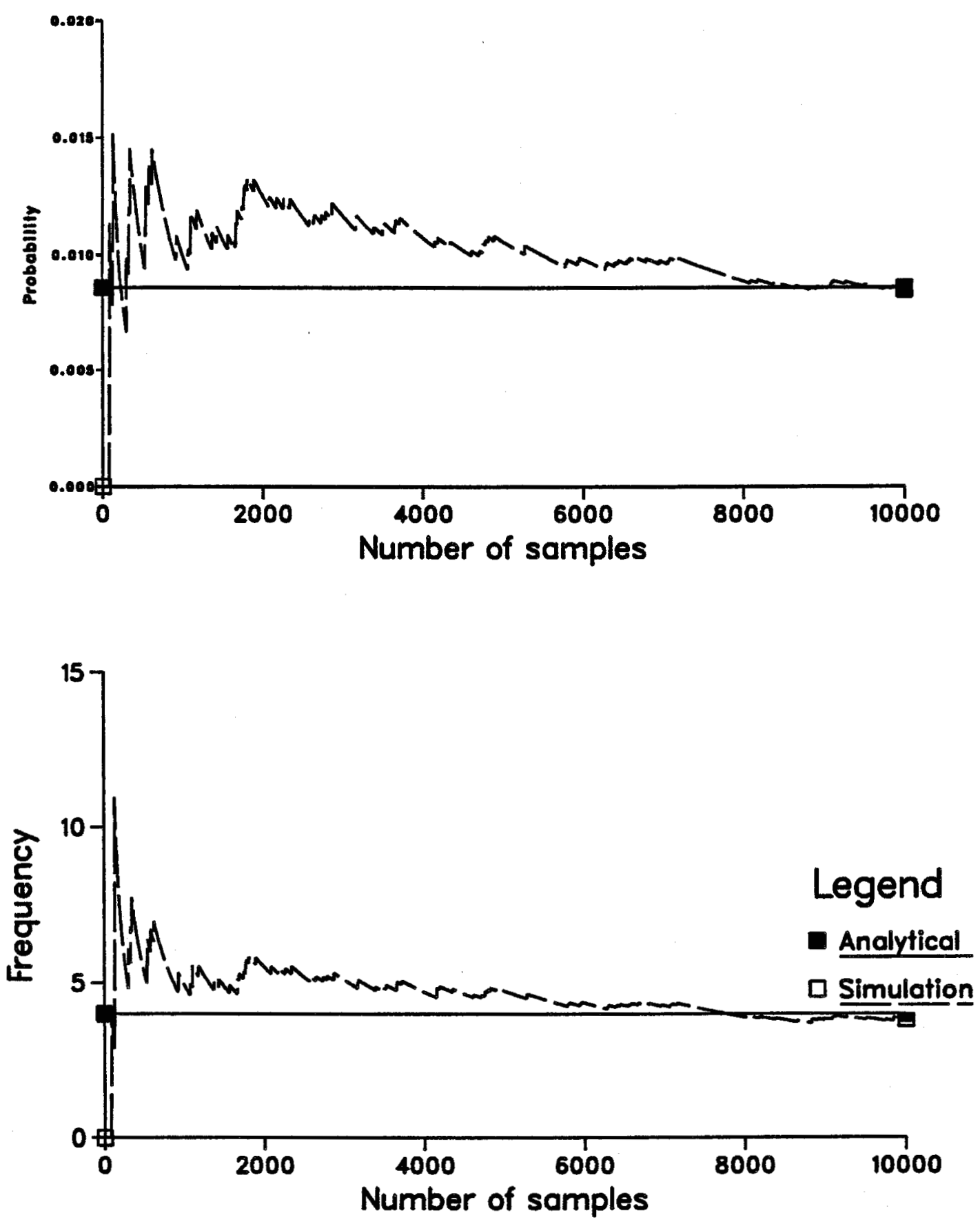

Figure 6.16: Probability and frequency of the extreme emergency state with number of samples using Method 2 - MRBTS 

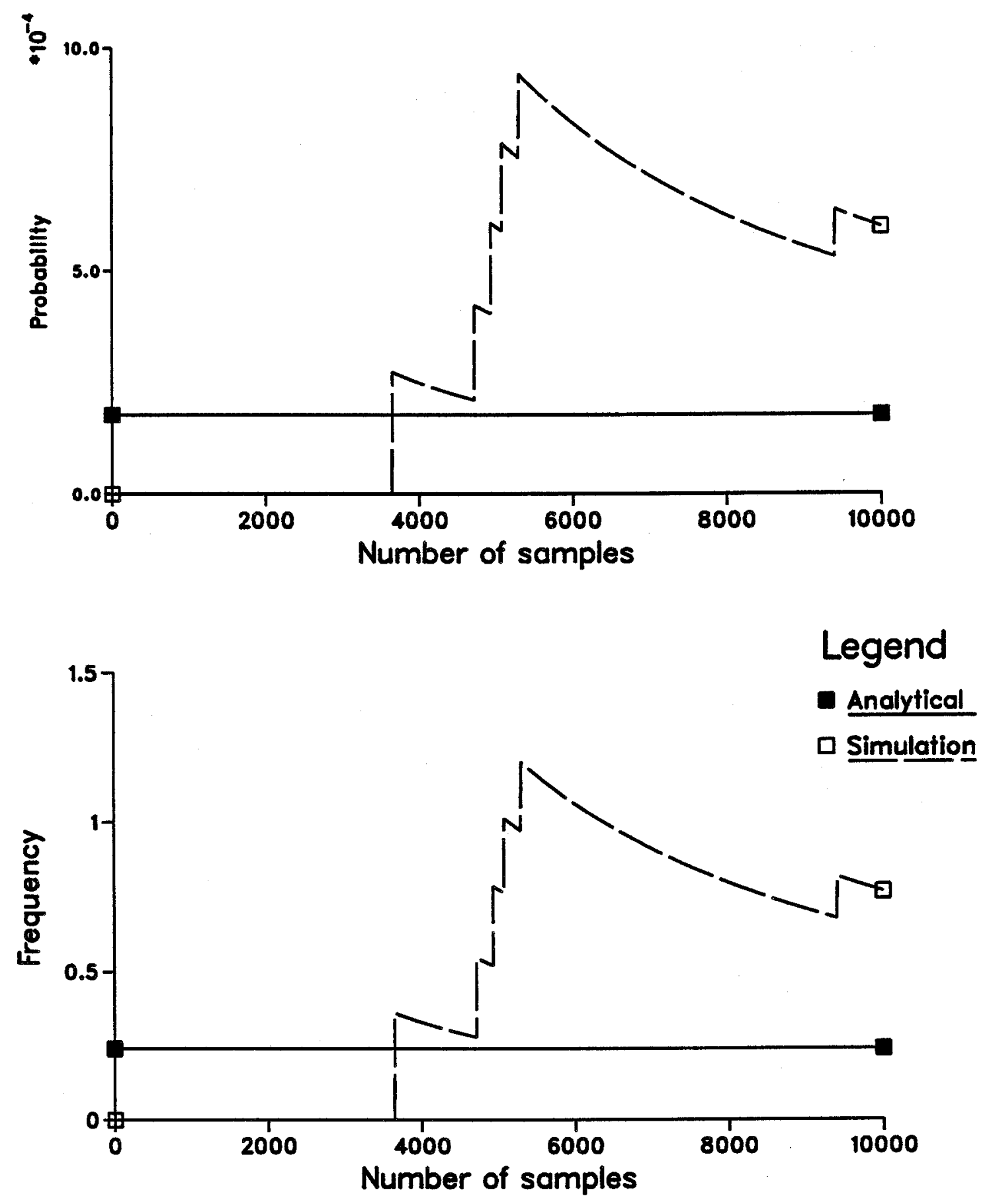

Figure 6.17: Probability and frequency of the no problem with number of samples using Method 2 - MRBTS 

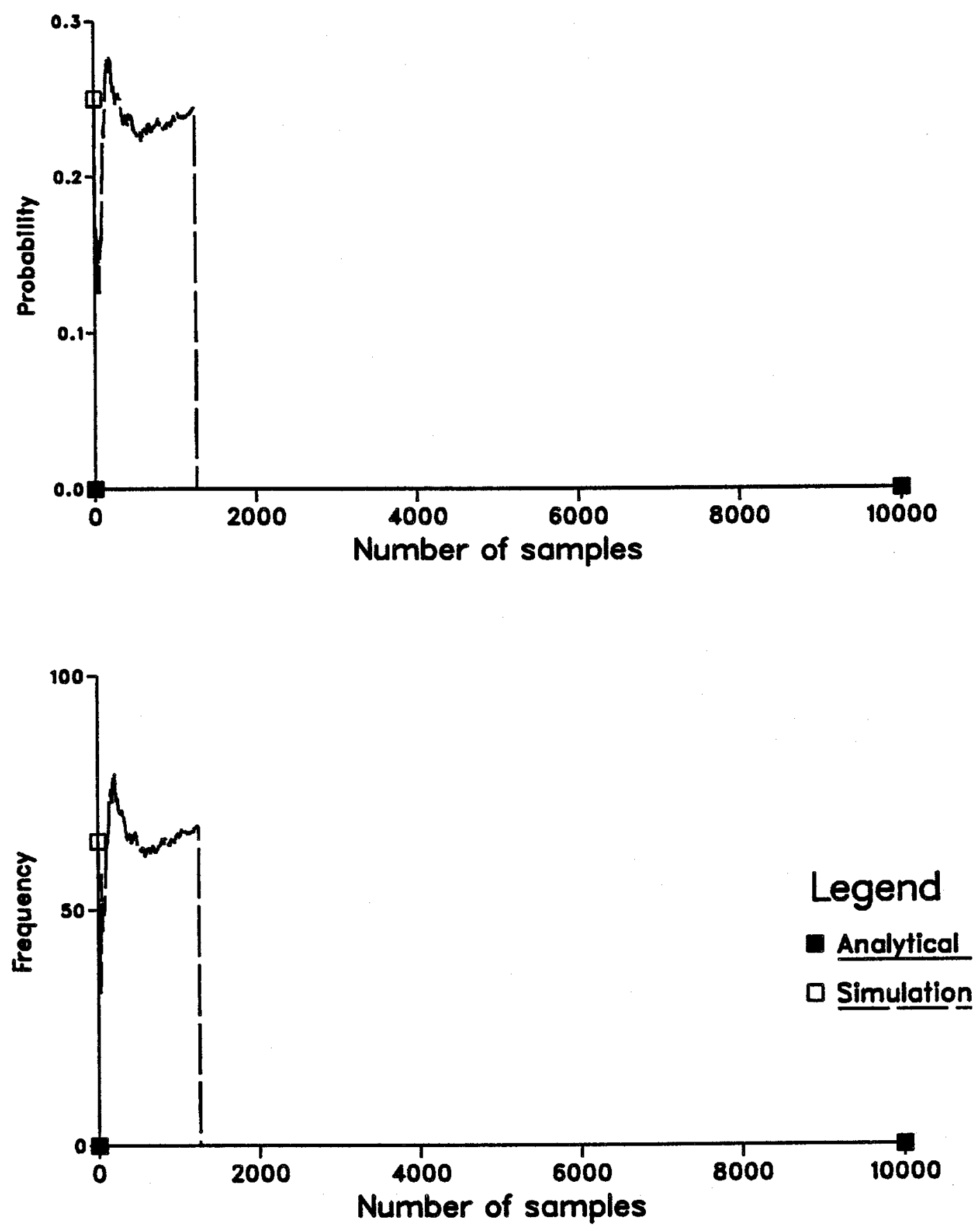

Figure 6.18: Probability and frequency of the normal state with number of samples using Method 2 - IEEE-RTS 

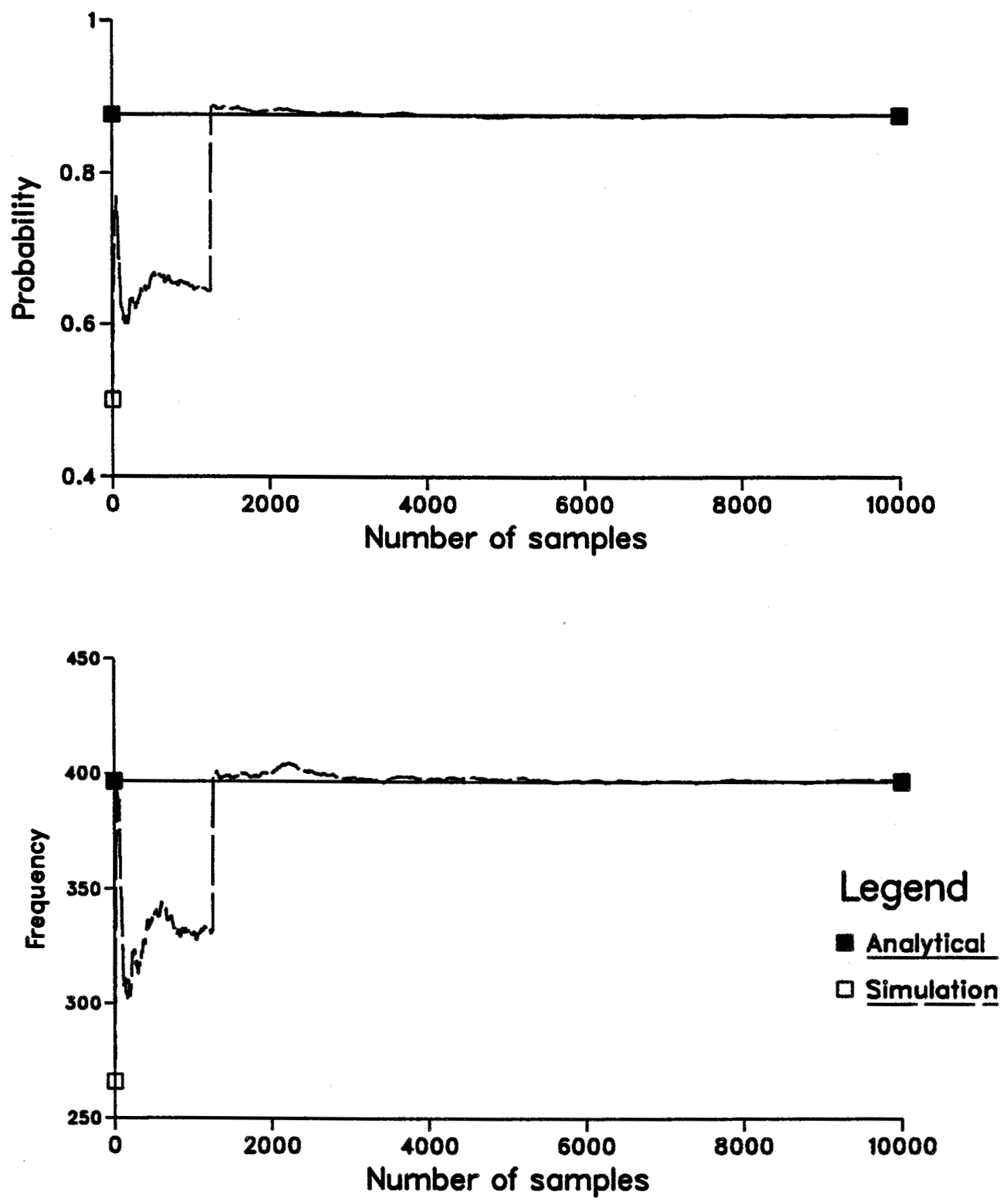

Figure 6.19: Probability and frequency of the alert state with number of samples using Method 2 - IEEE-RTS 

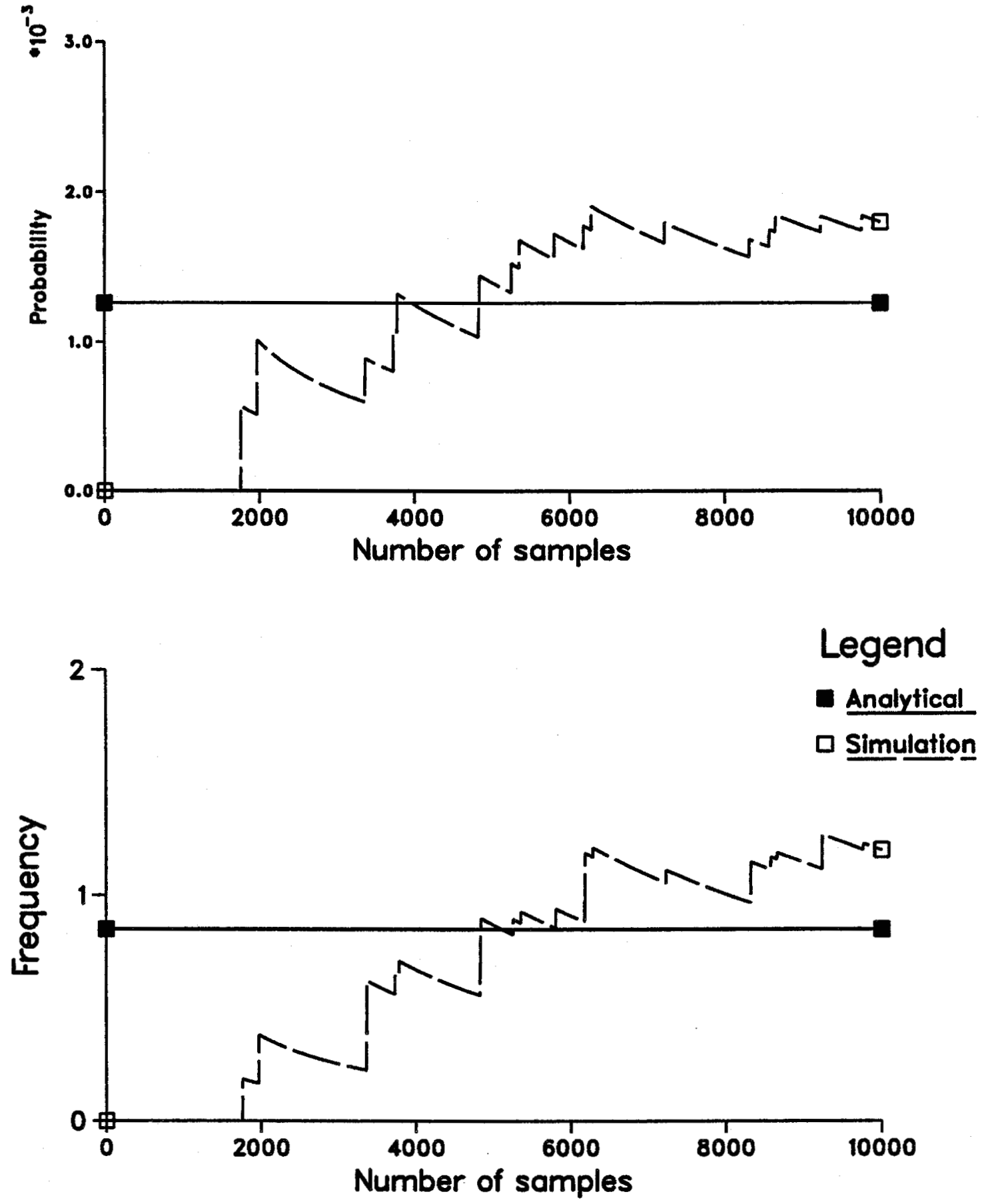

Figure 6.20: Probability and frequency of the emergency state with number of samples using Method 2 - IEEE-RTS 

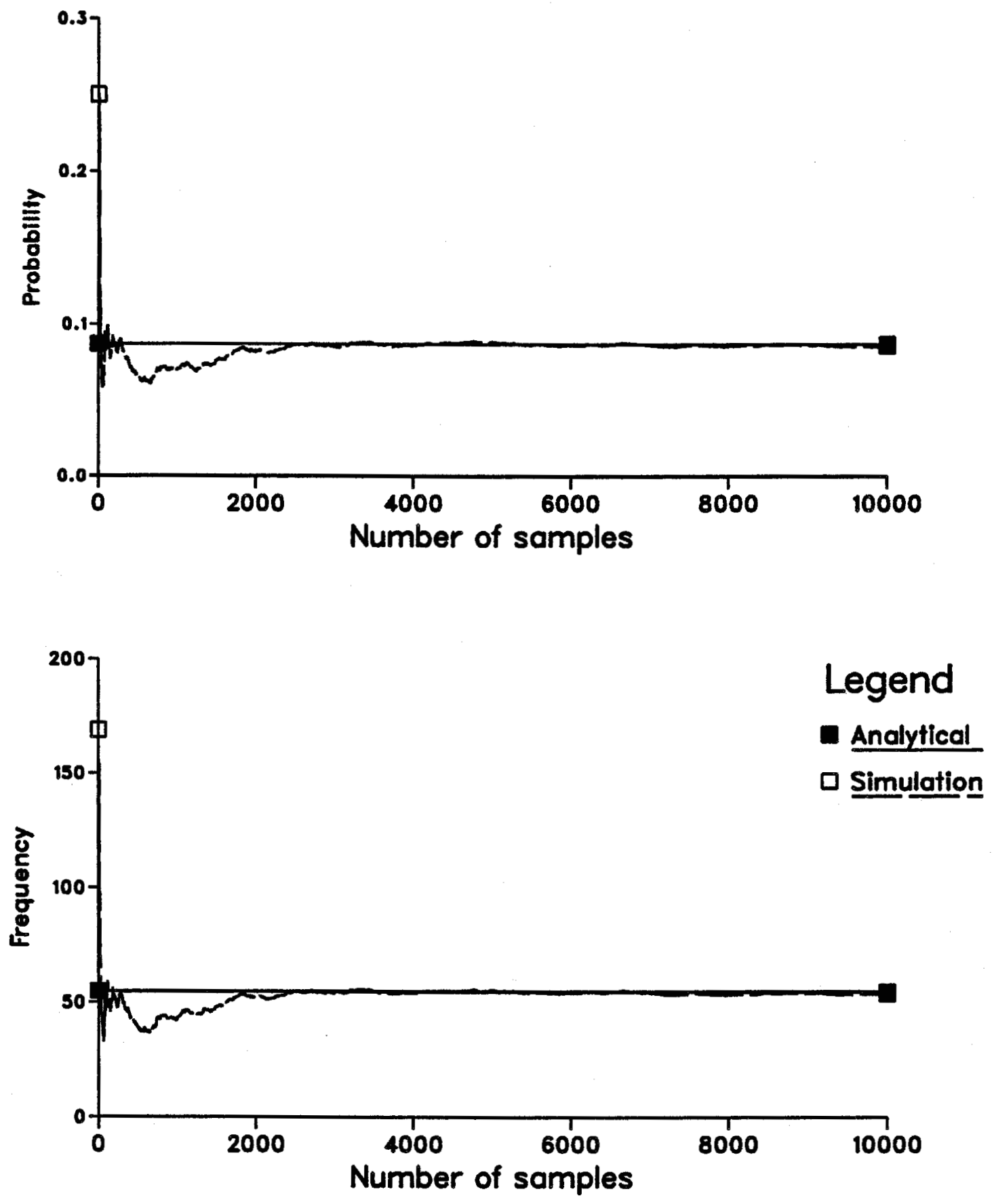

Figure 6.21: Probability and frequency of the extreme emergency state with number of samples using Method 2 - IEEE-RTS 

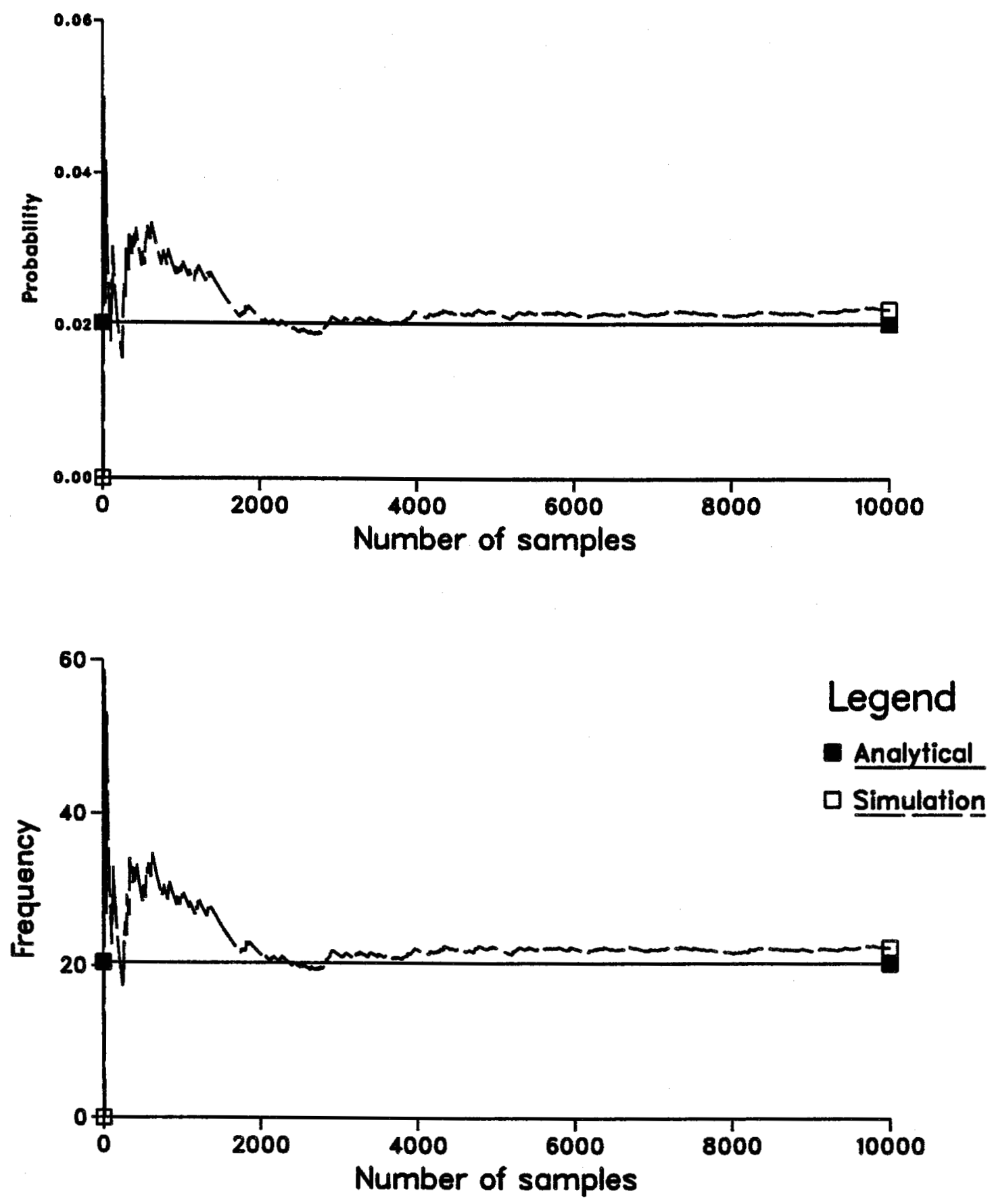

Figure 6.22: Probability and frequency of the no problem with number of samples using Method 2 - IEEE-RTS 
Table 6.2: Number of samples for different outage levels - MRBTS

\begin{tabular}{|c|c|c|c|c|c|c|}
\hline & Outage & $<-$ & & mber $\mathrm{O}$ & Samples & $-\cdots$ \\
\hline & $\begin{array}{l}0 \\
1 \\
2 \\
3 \\
4\end{array}$ & $\begin{array}{r}398 \\
88 \\
14 \\
0 \\
0\end{array}$ & $\begin{array}{r}789 \\
185 \\
25 \\
1 \\
0\end{array}$ & $\begin{array}{r}3969 \\
909 \\
113 \\
9 \\
0\end{array}$ & $\begin{array}{r}7941 \\
1845 \\
200 \\
14 \\
0\end{array}$ & $\begin{array}{r}39759 \\
9199 \\
977 \\
41 \\
4\end{array}$ \\
\hline Total & & 500 & 1000 & 5000 & 10000 & 50000 \\
\hline
\end{tabular}

Table 6.3: Number of samples for different outage levels - IEEE-RTS

\begin{tabular}{|c|c|c|c|c|c|c|}
\hline \multicolumn{2}{|c|}{$\begin{array}{l}\text { Outage } \\
\text { Level }\end{array}$} & $<----$ & \multicolumn{3}{|c|}{ Number Of Samples } & --...-.>> \\
\hline & $\begin{array}{l}0 \\
1 \\
2 \\
3 \\
4 \\
5 \\
6 \\
7\end{array}$ & $\begin{array}{r}111 \\
190 \\
125 \\
50 \\
18 \\
6 \\
0 \\
0\end{array}$ & $\begin{array}{r}229 \\
366 \\
248 \\
108 \\
38 \\
11 \\
0 \\
0\end{array}$ & $\begin{array}{r}1115 \\
1785 \\
1285 \\
566 \\
191 \\
51 \\
7 \\
0\end{array}$ & $\begin{array}{r}2288 \\
3543 \\
2496 \\
1182 \\
385 \\
90 \\
16 \\
0\end{array}$ & $\begin{array}{r}11495 \\
17645 \\
12583 \\
5873 \\
1843 \\
475 \\
81 \\
5\end{array}$ \\
\hline Total & & 500 & 1000 & 5000 & 10000 & 50000 \\
\hline
\end{tabular}

or lack of load curtailment can be used to determine whether the system is in the extreme emergency state or in one of the other three states. The above equation, therefore, can be simplified using the concept that the outcome $X_{i}$ of system situation $S_{i}$ will be zero or 1 as follows: 


$$
X_{i}=\left\{\begin{array}{ll}
0 & \text { if no load curtailment } \\
1 & \text { if load curtailment }
\end{array} \longrightarrow \frac{177}{N} \sum_{i=1}^{N} X_{i}^{2}=U\right.
$$

If $U$ is the expected value of $X_{i}$, then the variance $V(X)$ can be written as:

$$
\begin{aligned}
V(X) & =\frac{1}{N-1} \sum_{i=1}^{N}\left(X_{i}-U\right)^{2} \\
& =\frac{N}{N-1} U(1-U) .
\end{aligned}
$$

The relative variance coefficient can be therefore expressed as

$$
\begin{aligned}
\alpha & =\frac{S_{I}}{E(X)} \\
& =\sqrt{\frac{1-U}{(N-1) U}}
\end{aligned}
$$

The probabilities and frequencies of different system states for different values of $\alpha$ are presented in Table 6.4 for the MRBTS and in Table 6.5 for the IEEE-RTS. The number of samples required to obtain the results within $10 \%$ variability is 11376 and that for $5 \%$ variability is 45132 for the MRBTS. The CPU times for these two values of $\alpha$ are 9.21 seconds and 36.92 seconds on a Micro-VAX 3600 computer. For a sample size of 100000 , the variability is $3.46 \%$ and the corresponding CPU time is 83.14 seconds. There is no straight forward rule to select $\alpha$ which will provide satisfactory results within a reasonable number of samples and CPU time. The $\alpha$ equal to 0.05 gives a good compromise between results and CPU time for the MRBTS. The required sample for $\alpha \leq$ 0.05 is 45132 . In the following studies therefore, 50000 samples are used to obtain a reasonable estimate of the indices for the MRBTS. The CPU time increment for the IEEE-RTS is quite significant as the number of samples increases. The 10000 sampling for this system gives $\alpha \leq 0.0326$ and the required CPU time is also close to the analytical CPU time. This sampling number is therefore used for the IEEE-RTS studies described in the following sections. 
Table 6.4: Probability and frequency of different states for various $\alpha$-MRBTS

\begin{tabular}{|c|c|c|c|c|}
\hline & $\begin{array}{l}\text { System } \\
\text { States }\end{array}$ & $\begin{array}{c}\alpha \\
0.10\end{array}$ & $\begin{array}{c}\alpha \\
0.05\end{array}$ & $\begin{array}{c}\alpha \\
0.035\end{array}$ \\
\hline \multicolumn{5}{|l|}{ Probability } \\
\hline & Normal & 0.809951 & 0.813126 & 0.813210 \\
\hline & Alert & 0.180468 & 0.177479 & 0.177860 \\
\hline & Emergency & 0.000264 & 0.000199 & 0.000170 \\
\hline & Ext Emrg. & 0.008790 & 0.008796 & 0.008280 \\
\hline & No Prob & 0.000527 & 0.000355 & 0.000430 \\
\hline \multicolumn{5}{|l|}{ Frequency } \\
\hline & Normal & 50.241977 & 50.874490 & 50.700479 \\
\hline & Alert & 58.314636 & 57.624564 & 57.598640 \\
\hline & Emergency & 0.415257 & 0.303304 & 0.253872 \\
\hline & Ext Emrg. & 3.909449 & 4.052126 & 3.746726 \\
\hline & No Prob & 0.674957 & 0.470441 & 0.579084 \\
\hline & $\begin{array}{l}\text { Samples } \\
\text { CPU Time (Sec }\end{array}$ & $\begin{array}{r}11376 \\
9.21\end{array}$ & $\begin{array}{r}45132 \\
36.92\end{array}$ & $\begin{array}{r}100000 \\
83.14\end{array}$ \\
\hline
\end{tabular}

\subsection{VARIANCE REDUCTION TECHNIQUES}

The variance indicates the variability of the estimator around the expected value. Therefore, high variance essentially implies poor accuracy. A variance reduction technique (VRT) can be used to reduce the variance of the estimator by replacing the original sampling procedure by a new procedure that yields the same expected value but with a smaller variance. With proper application, a VRT can result in a greater precision, e.g., a smaller confidence interval for the same number of samples or, alternatively, achieve a prespecified precision with a smaller number of samples. Some VRT, such as importance sampling, changes the original sampling process completely. Other VRT, such as stratification after sampling, use the same sampling process as in the crude sampling, but after the sampling is over, they do not use the simple average but a more sophisticated estimator. 
Table 6.5: Probability and frequency of different states for various $\alpha$ - IEEE-RTS

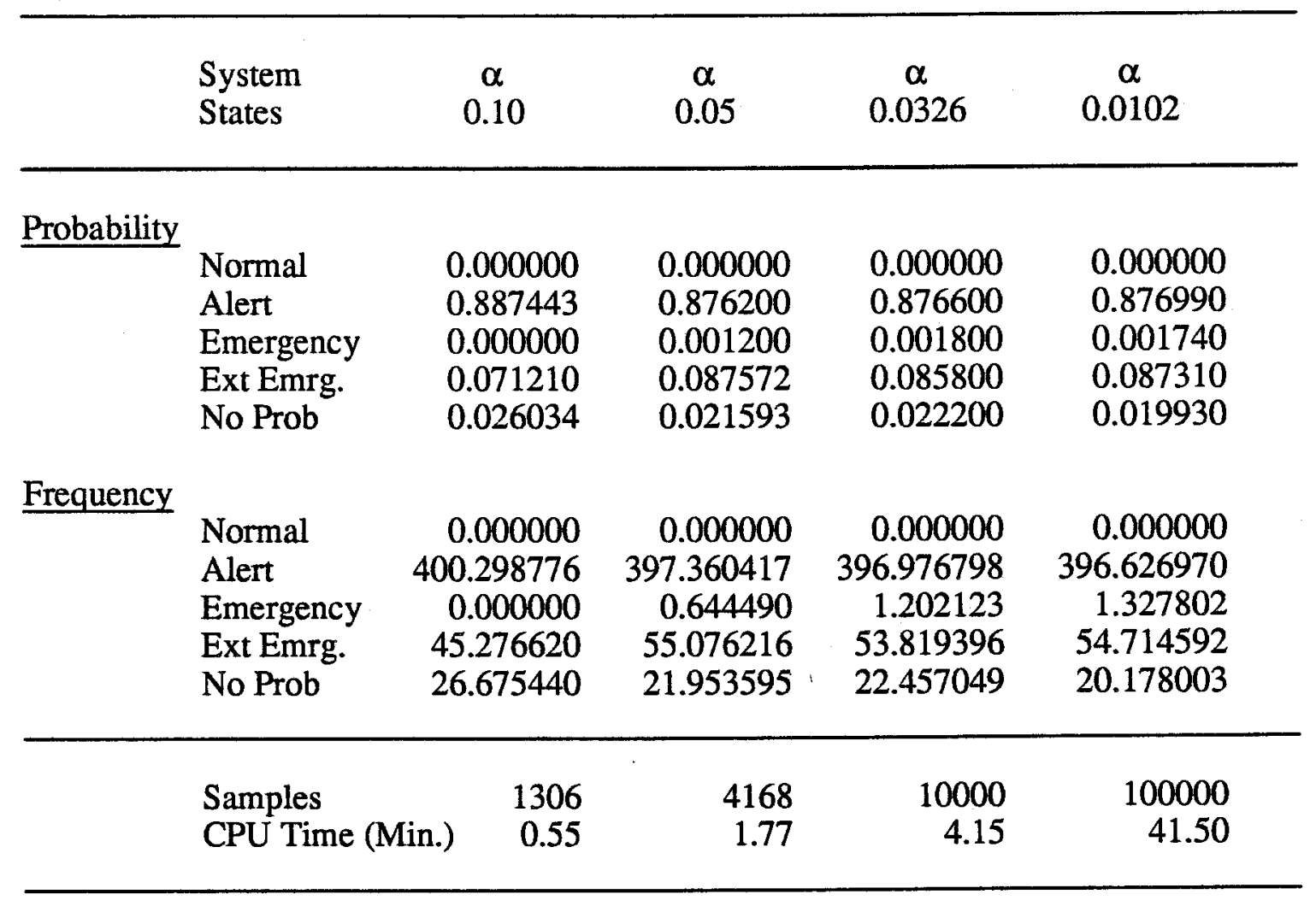

The implementation of a variance reduction technique may in itself require extra computing time and therefore should be considered carefully. The antithetic and stratification after sampling methods do not require much extra computation time and therefore have been investigated to study the effect of VRT on the simulation results.

\subsubsection{Antithetic Variates}

The antithetic VRT creates negative correlation between observations, generating one observation from the random number $r$ and the other observation from its 'antithetic' partner (1-r). For $X$, the mean value of the observation to be estimated, is given by

$$
X=\frac{1}{2}\left(X_{1}+X_{2}\right)
$$

where $X_{1}$ and $X_{2}$ are the response with random numbers $r_{i}$ and (1- $\left.r_{i}\right)$ respectively. The 
variance of the estimator is given by

$$
\operatorname{Var}(X)=\frac{1}{4}\left\{\operatorname{Var}\left(X_{1}\right)+\operatorname{Var}\left(X_{2}\right)+2 \operatorname{Cov}\left(X_{1}, X_{2}\right)\right\}
$$

Hence the variance of $X$ decreases since $\operatorname{Cov}\left(X_{1}, X_{2}\right)$ is a negative number. This method has been used and the results are presented in a later section.

\subsubsection{Stratification After Sampling}

This method is similar to the stratified sampling method except that in this case the number of observations in each stratum is not prefixed. It has been shown [106] that stratification after sampling is nearly as precise as proportionally stratified sampling provided that the samples per stratum are reasonably large, say $>20$. Stratification after sampling is attractive if it is impossible or difficult to fix the number of observations per stratum; therefore the stratum to which an observation belongs, is known only after the actual sampling.

This method consists of taking a simple, i.e. a nonstratified, sample of $\mathbf{n}$ observations. The new estimator $X$ is calculated using the formula given below instead of taking the simple average.

$$
\mathrm{X}=\sum_{k=1}^{J} \mathrm{P}_{\mathbf{k}} \mathrm{K}_{\mathrm{K}}
$$

where:

$$
\begin{aligned}
& \mathrm{Z}_{\mathrm{K}}=\sum_{i=1}^{n_{k}} \mathrm{X}_{\mathrm{ki}} / \mathrm{n}_{\mathrm{k}}, \\
& \mathrm{P}_{\mathrm{k}}=\text { probability of the } \mathrm{k}^{\text {th }} \text { stratum, } \\
& \mathrm{n}=\sum_{k=1}^{J} \mathrm{n}_{\mathrm{k}} \text { and } \\
& \mathrm{J}=\text { number of stratum. }
\end{aligned}
$$

In these studies, outages of generators, transmission lines and combined generator and transmission line outages are considered by random sampling. Therefore, five different stratum can be considered to represent the different component outages. These stratum are

1. no outage

2. only line outages 
3. only generator outages

4. generator and line outages

5. outages beyond the level considered

The probability of the above five stratum, $P_{k}$, can be calculated from the failure and repair rates of the components. The probabilities of different stratum for the two test systems are shown in Table 6.6. The variance of the estimator can be obtained using the

Table 6.6: Probabilities of different stratum for the two test systems

\begin{tabular}{ccc}
\hline Stratum & MRBTS & IEEE-RTS \\
\hline 1 & 0.79468942 & 0.23045127 \\
2 & 0.01831349 & 0.00593809 \\
3 & 0.18278383 & 0.73429596 \\
4 & 0.00418802 & 0.01512677 \\
5 & 0.00002524 & 0.01418791 \\
\hline Total & 1.0 & 1.0 \\
\hline
\end{tabular}

relations

$$
\begin{aligned}
& \operatorname{Var}(X)=\sum_{k=1}^{J} P_{k}{ }^{2} \frac{S_{k}^{2}}{n_{k}} \\
& S_{k}{ }^{2}=\frac{1}{n_{k}-1} \sum_{i=1}^{n_{k}}\left(X_{k i}-Z_{k}\right)^{2}
\end{aligned}
$$

\subsubsection{Results With The VRT}

The indices for different operating states have been calculated using the above two VRT. The indices for the MRBTS using the two methods are given in Table 6.7. The number of samples belonging to each stratum for the stratification after sampling method are shown in Table 6.8. The variances of the estimator with both the methods are less than that with out VRT for the same number of samples. It is very difficult to state which 
Table 6.7: Probability and frequency of different states without and with VRT MRBTS

$\begin{array}{llcc}\begin{array}{l}\text { System } \\ \text { States }\end{array} & \text { No VRT } & \text { Antithetic } & \begin{array}{c}\text { Stratified } \\ \text { After Sampling }\end{array}\end{array}$

Probability

$\begin{array}{llll}\text { Normal } & 0.812880 & 0.811100 & 0.812430 \\ \text { Alert } & 0.177720 & 0.179840 & 0.178170 \\ \text { Emergency } & 0.000180 & 0.000180 & 0.000179 \\ \text { Ext Emrg. } & 0.008820 & 0.008560 & 0.008813 \\ \text { No Prob } & 0.000340 & 0.000300 & 0.000325\end{array}$

Frequency

$\begin{array}{lrrr}\text { Normal } & 50.839961 & 50.674853 & 50.839961 \\ \text { Alert } & 57.503811 & 58.684511 & 57.503811 \\ \text { Emergency } & 0.273775 & 0.260258 & 0.273775 \\ \text { Ext Emrg. } & 4.015106 & 3.802385 & 4.015106 \\ \text { No Prob } & 0.450222 & 0.394321 & 0.450222\end{array}$

Table 6.8: Number of samples in different system states from different stratum MRBTS

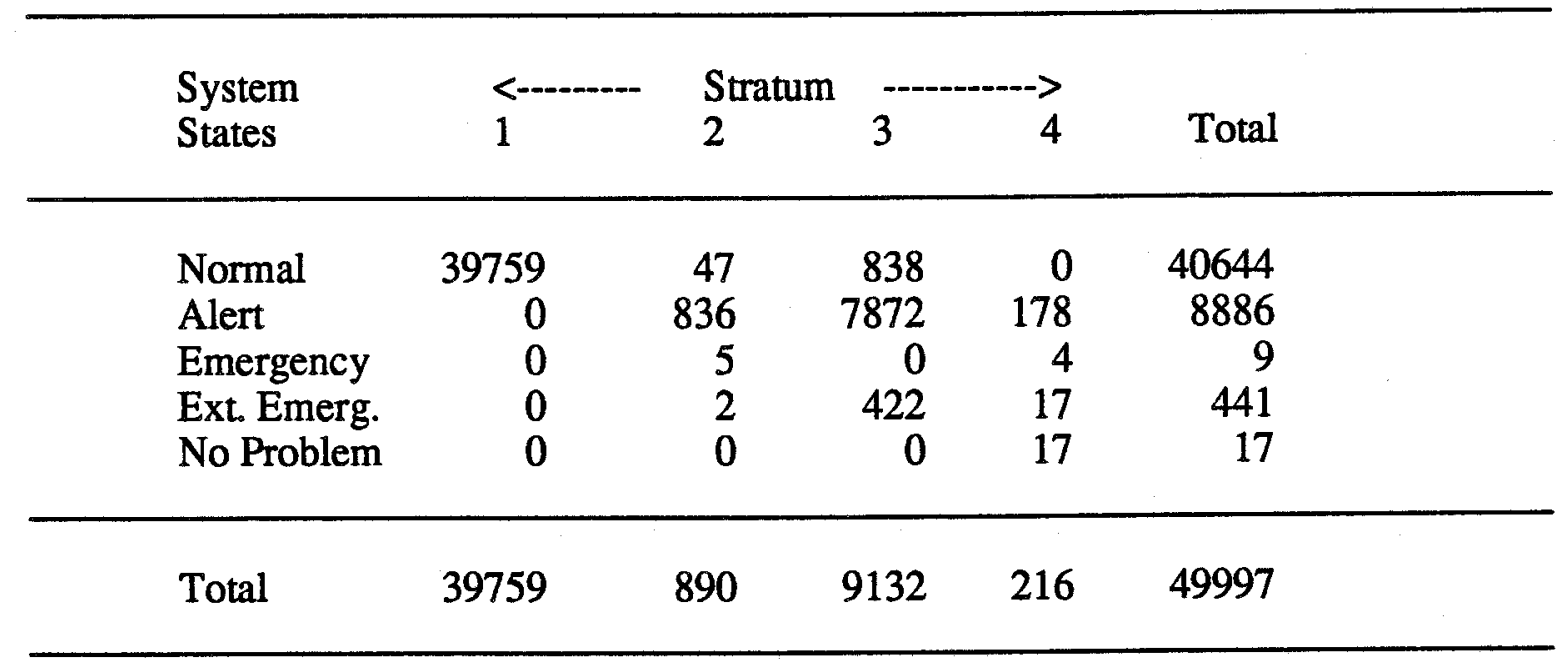

method is better than the other. The justification of whether to use VRT or not can be measured by the efficiency of the simulation method. The efficiency can be measured using 


$$
\eta=\frac{t_{1} \operatorname{Var}\left(X_{1}\right)}{t_{2} \operatorname{Var}\left(X_{2}\right)}
$$

where $X_{1}$ and $X_{2}$ are the estimates produced by the methods and $t_{1}$ and $t_{2}$ are the computation times required for evaluating $X_{1}$ and $X_{2}$ respectively. The first method is more efficient than the second method when $\eta>1$. The CPU times and the variances of the estimates are shown in Table 6.9. In the case of the MRBTS, the efficiency of the antithetic method is 2.1186 and that of the stratification after sampling is 1.0563 over the simple method. This suggests that the antithetic variance reduction technique is more suitable than the stratification after sampling method with respect to the calculation of indices for the extreme emergency state. It should be noted that the efficiency of the methods with respect to other system states may lead to different conclusions. Similar

Table 6.9: Variance of estimation and CPU time for the two test systems

\begin{tabular}{|c|c|c|c|}
\hline & Method & Variance & $\begin{array}{c}\text { CPU Time On } \\
\text { Micro-VAX } 3600\end{array}$ \\
\hline \multicolumn{4}{|l|}{ MRBTS } \\
\hline & With out VRT & $1.75 \mathrm{E}-7$ & 38.86 Seconds \\
\hline & Antithetic & $8.50 \mathrm{E}-8$ & 37.74 Seconds \\
\hline & Stratification & $1.68 \mathrm{E}-7$ & 38.32 Seconds \\
\hline \multicolumn{4}{|c|}{ IEEE-RTS } \\
\hline & With out VRT & $7.84 \mathrm{E}-6$ & 4.15 Minutes \\
\hline & Antithetic & $3.69 \mathrm{E}-6$ & 4.13 Minutes \\
\hline & Stratification & $7.34 \mathrm{E}-6$ & 4.15 Minutes \\
\hline
\end{tabular}

indices for the IEEE-RTS are shown in Table 6.10 and Table 6.11. The efficiency of the antithetic VRT is 2.1349 and that of the stratification after sampling is 1.0668 over the simple method. In this case the antithetic VRT is also more efficient than the stratification after sampling method with respect to the extreme emergency state. 
Table 6.10: Probability and frequency of different states without and with VRT IEEE-RTS

$\begin{array}{lllc}\begin{array}{l}\text { System } \\ \text { States }\end{array} & \text { No VRT } & \text { Antithetic } & \begin{array}{c}\text { Stratified } \\ \text { After Sampling }\end{array}\end{array}$

Probability

$\begin{array}{llll}\text { Normal } & 0.000000 & 0.000000 & 0.000000 \\ \text { Alert } & 0.876600 & 0.875700 & 0.866372 \\ \text { Emergency } & 0.001800 & 0.001900 & 0.001628 \\ \text { Ext Emrg. } & 0.085800 & 0.084700 & 0.084255 \\ \text { No Prob. } & 0.022200 & 0.020700 & 0.020906\end{array}$

Frequency

$\begin{array}{lrrr}\text { Normal } & 0.000000 & 0.000000 & 0.000000 \\ \text { Alert } & 396.976798 & 398.371209 & 396.976798 \\ \text { Emergency } & 1.202123 & 0.998096 & 1.202123 \\ \text { Ext Emrg. } & 53.819396 & 53.025271 & 53.819396 \\ \text { No Prob. } & 22.457049 & 21.104319 & 22.457049\end{array}$

Table 6.11: Number of samples in different system states from different stratum IEEE-RTS

\begin{tabular}{|c|c|c|c|c|c|}
\hline $\begin{array}{l}\text { System } \\
\text { States }\end{array}$ & 1 & $\begin{array}{l}\text { Stratum } \\
2\end{array}$ & n 3 & $->$ & Total \\
\hline Normal & 2288 & 1 & 132 & 1 & 2422 \\
\hline Alert & 0 & 42 & 6225 & 77 & 6344 \\
\hline Emergency & 0 & 5 & 0 & 13 & 18 \\
\hline Ext. Emerg. & 0 & 3 & 849 & 6 & 858 \\
\hline No Problem & 0 & 0 & 171 & 51 & 222 \\
\hline Total & 2288 & 51 & 7377 & 148 & 9864 \\
\hline
\end{tabular}




\subsection{ANNUAL INDICES}

As noted earlier, the annualized indices give a pessimistic apprisal since the load does not stay at the peak level throughout a year. The annual indices provide a more representative picture of the system. In order to calculate a complete set of annual indices, the 8760 hourly peak load model should be used in the studies. The examples presented in this section however, were conducted using the seven step load model described in Chapter 4 in order to illustrate the simulation process. The load is selected randomly from the seven step load model. A flow chart similar to that of Method 2 was utilized with the appropriate modification to randomly select the load levels. The probability of different load levels for the two test systems for 10000 and 50000 samples are shown in Table 6.12. The annual indices for the two test systems and the contribution

Table 6.12: Probability of different load levels using simulation

\begin{tabular}{rlr}
\hline Load & \multicolumn{2}{c}{ Samples $--->$} \\
Step & 10000 & 50000 \\
& & \\
\hline $40 \%$ & 0.037700 & 0.036740 \\
$50 \%$ & 0.227400 & 0.226260 \\
$60 \%$ & 0.216500 & 0.217280 \\
$70 \%$ & 0.230700 & 0.232820 \\
$80 \%$ & 0.163900 & 0.164540 \\
$90 \%$ & 0.109900 & 0.109600 \\
$100 \%$ & 0.013900 & 0.012760 \\
\hline
\end{tabular}

to the annual indices from each load level are shown in Tables 6.13 to 6.16. It can be seen from these tables that both the probabilities and frequencies of the normal state for the two test systems are much higher than the corresponding values of the annualized indices calculated using the peak load demand. The indices for the extreme emergency state are lower than those obtained in the annualized case. The reason for this is that at lower load levels, both the systems have enough generation and in most cases require no load curtailments to alleviate a system problem due to contingencies. It can also be seen from these tables that the contributions to the annual indices from the middle range of the load levels are very significant compared to that of the peak load level. 
Table 6.13: Contribution of different load levels to state probability - MRBTS

\begin{tabular}{cccccc}
\hline $\begin{array}{c}\text { Load } \\
\text { Step }\end{array}$ & Normal & Alert & $\begin{array}{c}\text { Contribution to } \\
\text { Emergency }\end{array}$ & $\begin{array}{c}\text { Ext. } \\
\text { Emergency }\end{array}$ & $\begin{array}{c}\text { No } \\
\text { Problem }\end{array}$ \\
\hline & & & & & \\
$40 \%$ & 0.036057 & 0.000660 & 0.000004 & 0.000000 & 0.000016 \\
$50 \%$ & 0.222043 & 0.004077 & 0.000027 & 0.000000 & 0.000100 \\
$60 \%$ & 0.213204 & 0.003941 & 0.000026 & 0.000000 & 0.000096 \\
$70 \%$ & 0.228001 & 0.004675 & 0.000028 & 0.000000 & 0.000102 \\
$80 \%$ & 0.160236 & 0.004173 & 0.000020 & 0.000033 & 0.000069 \\
$90 \%$ & 0.098618 & 0.010671 & 0.000011 & 0.000252 & 0.000042 \\
$100 \%$ & 0.010372 & 0.002268 & 0.000002 & 0.000113 & 0.000004 \\
Annual & 0.968530 & 0.030465 & 0.000119 & 0.000398 & 0.000429 \\
\hline
\end{tabular}

Table 6.14: Contribution of different load levels to state frequency - MRBTS

\begin{tabular}{|c|c|c|c|c|c|}
\hline \multirow{2}{*}{$\begin{array}{l}\text { Load } \\
\text { Step }\end{array}$} & \multirow{2}{*}{ Normal } & \multirow[b]{2}{*}{ Alert } & \multirow{2}{*}{$\begin{array}{l}\text { Contribution to } \\
\text { Emergency }\end{array}$} & \multirow{2}{*}{$\begin{array}{l}\text { Ext. } \\
\text { Emergency }\end{array}$} & \multirow[b]{2}{*}{$\begin{array}{c}\text { No } \\
\text { Problem }\end{array}$} \\
\hline & & & & & \\
\hline $40 \%$ & 3.490253 & 0.634956 & 0.008075 & 0.000000 & 0.021383 \\
\hline $50 \%$ & 21.479807 & 3.924922 & 0.049729 & 0.000000 & 0.131686 \\
\hline $60 \%$ & 20.620898 & 3.775546 & 0.047755 & 0.000000 & 0.126459 \\
\hline $70 \%$ & 21.909053 & 4.232239 & 0.051170 & 0.000000 & 0.135504 \\
\hline $80 \%$ & 15.180685 & 3.276910 & 0.036163 & 0.019675 & 0.093231 \\
\hline $90 \%$ & 7.850264 & 4.344046 & 0.019730 & 0.124818 & 0.055033 \\
\hline $100 \%$ & 0.648718 & 0.733749 & 0.003493 & 0.051233 & 0.005745 \\
\hline Annual & 91.179678 & 20.922368 & 0.216116 & 0.195725 & 0.569040 \\
\hline
\end{tabular}


Table 6.15: Contribution of different load levels to state probability - IEEE-RTS

\begin{tabular}{|c|c|c|c|c|c|}
\hline \multirow{2}{*}{$\begin{array}{l}\text { Load } \\
\text { Step }\end{array}$} & \multicolumn{2}{|c|}{$<-$} & Contribution to & \\
\hline & Normal & Alert & Emergency & $\begin{array}{c}\text { Ext. } \\
\text { Emergency }\end{array}$ & $\begin{array}{c}\text { No } \\
\text { Problem }\end{array}$ \\
\hline $40 \%$ & 0.035106 & 0.000532 & 0.000004 & 0.000000 & 0.001546 \\
\hline $50 \%$ & 0.211755 & 0.003206 & 0.000023 & 0.000000 & 0.009323 \\
\hline $60 \%$ & 0.201605 & 0.003053 & 0.000022 & 0.000000 & 0.008877 \\
\hline $70 \%$ & 0.213859 & 0.004222 & 0.000023 & 0.000000 & 0.009459 \\
\hline $80 \%$ & 0.148739 & 0.006146 & 0.000016 & 0.000148 & 0.006622 \\
\hline $90 \%$ & 0.089569 & 0.013650 & 0.000011 & 0.001242 & 0.003934 \\
\hline $100 \%$ & 0.006944 & 0.005271 & 0.000001 & 0.001180 & 0.000314 \\
\hline Annual & 0.907577 & 0.036079 & 0.000100 & 0.002569 & 0.040074 \\
\hline
\end{tabular}

Table 6.16: Contribution of different load levels to state frequency - IEEE-RTS

\begin{tabular}{|c|c|c|c|c|c|}
\hline \multirow{2}{*}{$\begin{array}{l}\text { Load } \\
\text { Step }\end{array}$} & \multirow{2}{*}{ Normal } & \multirow[b]{2}{*}{ Alert } & \multirow{2}{*}{$\begin{array}{l}\text { Contribution to } \\
\text { Emergency }\end{array}$} & \multirow{2}{*}{$\begin{array}{l}\text { D } \\
\text { Ext. } \\
\text { Emergency }\end{array}$} & \multirow[b]{2}{*}{$\begin{array}{c}\text { No } \\
\text { Problem }\end{array}$} \\
\hline & & & & & \\
\hline $40 \%$ & 15.991117 & 0.443551 & 0.004625 & 0.000000 & 1.441458 \\
\hline $50 \%$ & 96.455705 & 2.675425 & 0.027897 & 0.000000 & 8.694631 \\
\hline $60 \%$ & 91.832278 & 2.547184 & 0.026559 & 0.000000 & 8.277870 \\
\hline $70 \%$ & 97.506058 & 3.063650 & 0.028301 & 0.000000 & 8.820806 \\
\hline $80 \%$ & 67.584006 & 3.843725 & 0.020107 & 0.085550 & 6.202824 \\
\hline $90 \%$ & 39.058184 & 8.474767 & 0.013482 & 0.807487 & 3.770605 \\
\hline $100 \%$ & 2.730228 & 2.803293 & 0.001705 & 0.739973 & 0.317439 \\
\hline Annual & 411.157576 & 23.851594 & 0.122676 & 1.633010 & 37.525632 \\
\hline
\end{tabular}




\subsection{SUMMARY}

The two most popular methods for composite system reliability evaluation are the enumeration method and the Monte Carlo simulation approach. These two methods have been combined to form a hybrid technique to evaluate the different system operating states. Two approaches have been investigated and it has been found that Method 1 does not provide satisfactory results. Method 2, however, provides basically similar indices to those obtained by the analytical approach when a suitable number of samples are used. The accuracy of the estimation of a reliability index using simulation depends upon the number of samples and the frequency of the occurrence of the events which affect the index. For example, a good estimate of the probability of the extreme emergency state will require a large number of samples if events which cause load curtailment occur very infrequently. There is no definite rule to determine the number of samples that should be specified as being sufficient for all systems. Usually, smaller systems require much higher sampling than larger systems. The number of samples required also depends on the reliability of the system. A reliable system requires more trials than an unreliable system of the same size. The relative variance coefficient has been used to limit the number of samples for the systems analyzed. It was found that the convergence for the MRBTS is slower than that for the IEEE-RTS with respect to the extreme emergency state. For the MRBTS, 50000 samples give reasonable results whereas 10000 samples are required for the IEEE-RTS.

The accuracy in estimating a reliability index is proportional to the square root of the number of samples. Therefore, four times as many samples are required to reduce the random error by one half. This is the main drawback of the Monte Carlo simulation method. The precision of estimation for the same number of samples or, conversely the number of samples for the same precision can be reduced by using variance reduction techniques. Stratification after sampling and antithetic VRTs have been considered in this chapter. It has been found that antithetic VRT gives more satisfactory results in terms of efficiency for the extreme emergency state. The conclusion, however, could be different for the other system states. Both annualized and annual indices have been calculated and presented in this chapter. 
The indices for different operating states can be utilized in planning and expansion analysis of the system. A risk index can be calculated for this purpose and compared with a given acceptable system risk in order to justify the addition of generation and transmission facilities and the location within the system of these additional facilities. This concept is discussed in the next chapter. 


\section{UTILIZATION OF THE RELIABILITY ASSESSMENT CONCEPTS FOR SYSTEM PLANNING}

\subsection{INTRODUCTION}

The operating domain of a composite power system has been divided into different states in terms of the degree with which these states satisfy both adequacy and security constraints. Mathematical models were developed and discussed in the previous chapters to quantify these operating states. In the normal and alert states, the system does not violate any operating constraints. A system operating objective, therefore, is to operate with a high probability within these two states. The complement of the sum of these two state probabilities can be defined as a risk index and utilized as a criterion in planning the expansion of the system to meet future load growth.

An electric power system planner is concerned both with the level of predicted reliability and the investment and operating alternatives associated with meeting the desired level. In this thesis, these alternatives consist of generation and transmission facilities. Distribution elements are not considered. A generation expansion plan must provide the electric utility with the capability of meeting customer needs for a reasonably priced, reliable and quality electric energy supply. Selecting a particular generation system expansion plan from the many available to an electric utility is complicated, especially since all utilities strive for the best strategy in an environment of uncertainty. This chapter will first illustrate a technique which can be used to determine whether a generation expansion plan satisfies a desired level of reliability defined by the risk index determined from the probabilities of the normal and the alert states. The objective of this chapter is to illustrate the utilization of this new risk index in conventional generating and transmission system planning. 
Transmission system planning usually follows generation planning because the construction time for new transmission facilities is usually much shorter than that required for generation facilities and also because transmission planning depends on a specific knowledge of the location and capacity of both generation and load centers. When new load centers develop or new generation facilities are added, they are in effect connected to the existing system through zero capacity elements, creating an overload situation resolved only by adding lines of appropriate capacity to interconnect the new elements to the system or to reinforce the existing system. The main problem is to determine the capacities and locations of the new lines or capacity additions to the existing lines, so that no overload lines or voltage problems will be produced under normal steady-state conditions for some future forecast load levels. In order to maintain a specified risk index for a given power system with increasing system load, it is necessary to add generating units and/or transmission lines to the existing system. This chapter will illustrate using the two test systems how the defined risk indices can be utilized in composite system expansion planning

\subsection{EXPANSION PLANNING IN THE MRBTS}

System adequacy depends upon many factors such as the amount of installed capacity, sizes of the various generating units and their availabilities, system load, transmission line facilities etc.. In an HLI study, system expansion is performed by adding appropriate generation facilities to satisfy the load without violating the accepted risk. Both deterministic and probabilistic methods have been applied to determine the required reserve capacity in a system. The most common deterministic criterion relates the reserve margin to the size of the largest generating unit or to some percentage of the peak demand [2]. The most popular probabilistic criterion is the LOLE index [2]. Transmission facilities are not normally included in this assessment. The objective of this section is to demonstrate the use of the normal and alert state probabilities by considering the generation and major transmission facilities required to satisfy a certain risk level defined for the system. The risk factor utilized in this research is calculated using the formula given below:

$$
\text { Risk }=1.0-P_{n}-P_{a}-P_{n p}
$$


This risk factor has been designated as the Composite System Operating State Risk (CSOSR). As noted earlier, a system operates without violating any constraints in both the normal and the alert states. A system planner, therefore, may wish to have the system remains in these states with a high probability. The probabilities of 'no problem' are the probabilities of those extreme outages which do not create system problems and therefore these outages belong to either the normal or the alert states. The sum of the three probabilities of the normal, alert and no problem states therefore, represent an assessment of the favourable condition associated with the system. The complement of the sum of these three probabilities represents the unfavourable condition and hence constitutes the risk level for the system. This risk index is utilized in this chapter to justify the addition of generation and transmission facilities with system load growth. Other possibilities for defining the unfavourable condition also exist. The system risk could be simply defined as the probability of the system residing in the extreme emergency state as this state is the only state involving load curtailment. A CSOSR level less than or equal to 0.01 is used for the MRBTS studies.

\subsubsection{Generation Capacity Addition}

The objective behind this study is to determine when additional generation facilities must be added as the system load grows. The additional generation unit parameters [91] are given in Table 7.1. The base system peak load for this system is $185 \mathrm{MW}$. The variation of the CSOSR with percentage load increments is shown in Figure 7.1. It can be seen from this figure that for up to $2 \%$ increase in the base peak load, the system operates within the operating CSOSR of 0.01 . When the load increment is more than $2 \%$ of the base peak load, the system risk becomes more than 0.01 which indicates that additional generation is required to satisfy the increased load within the specified CSOSR. The actual location of the generation facilities is not a factor in an HLI assessment. Remote facilities can be modelled including their associated radial transmission if required [2]. In the case of HLII i.e. composite system assessment, the location of the generation facilities becomes important together with the ability of the associated transmission lines to move the generated energy to the load points. It is 
Table 7.1: Additional generating units for the MRBTS

\begin{tabular}{cccc}
\hline $\begin{array}{c}\text { Capacity } \\
(\mathrm{MW})\end{array}$ & FOR & $\begin{array}{c}\text { MTTF } \\
\text { (Hours) }\end{array}$ & $\begin{array}{c}\text { MTTR } \\
\text { (Hours) }\end{array}$ \\
\hline 10 & 0.12 & 550 & 75 \\
\hline
\end{tabular}

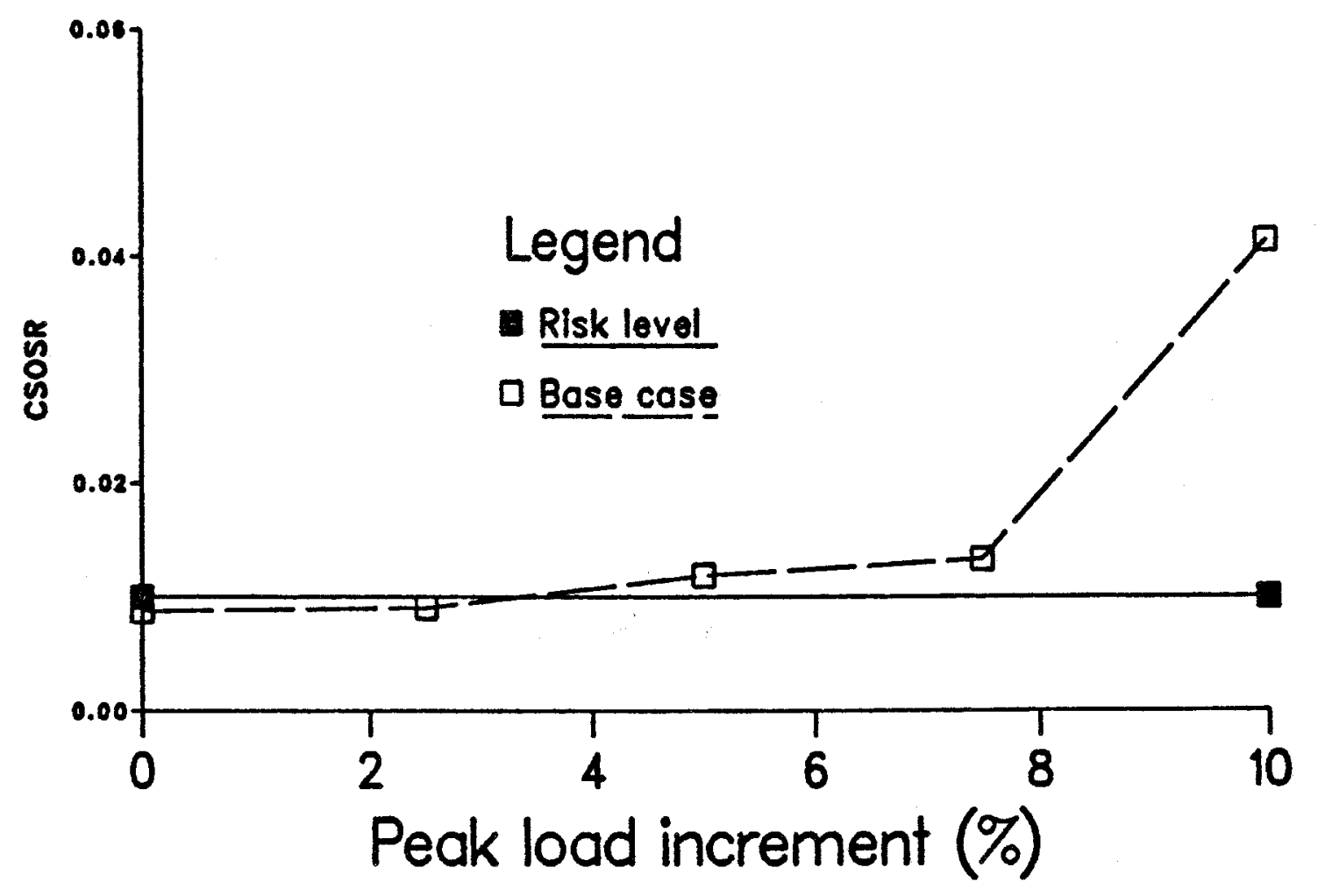

Figure 7.1: CSOSR variation with peak load increment - MRBTS

assumed that additional generation can be added to any generator bus in the system. This system has two generator buses, namely bus 1 and bus 2 . In order to find which bus is the most suitable for addition, generators are added to each bus one at a time and studies conducted for a load increment of up to $10 \%$ of the base peak load. Figure 7.2 shows the generation addition at bus 1 and Figure 7.3 shows the effect of adding generation at bus 2. It can be seen from these figures that with the addition of the first generator, an 


\section{Why Figme and 3 are completely saine}

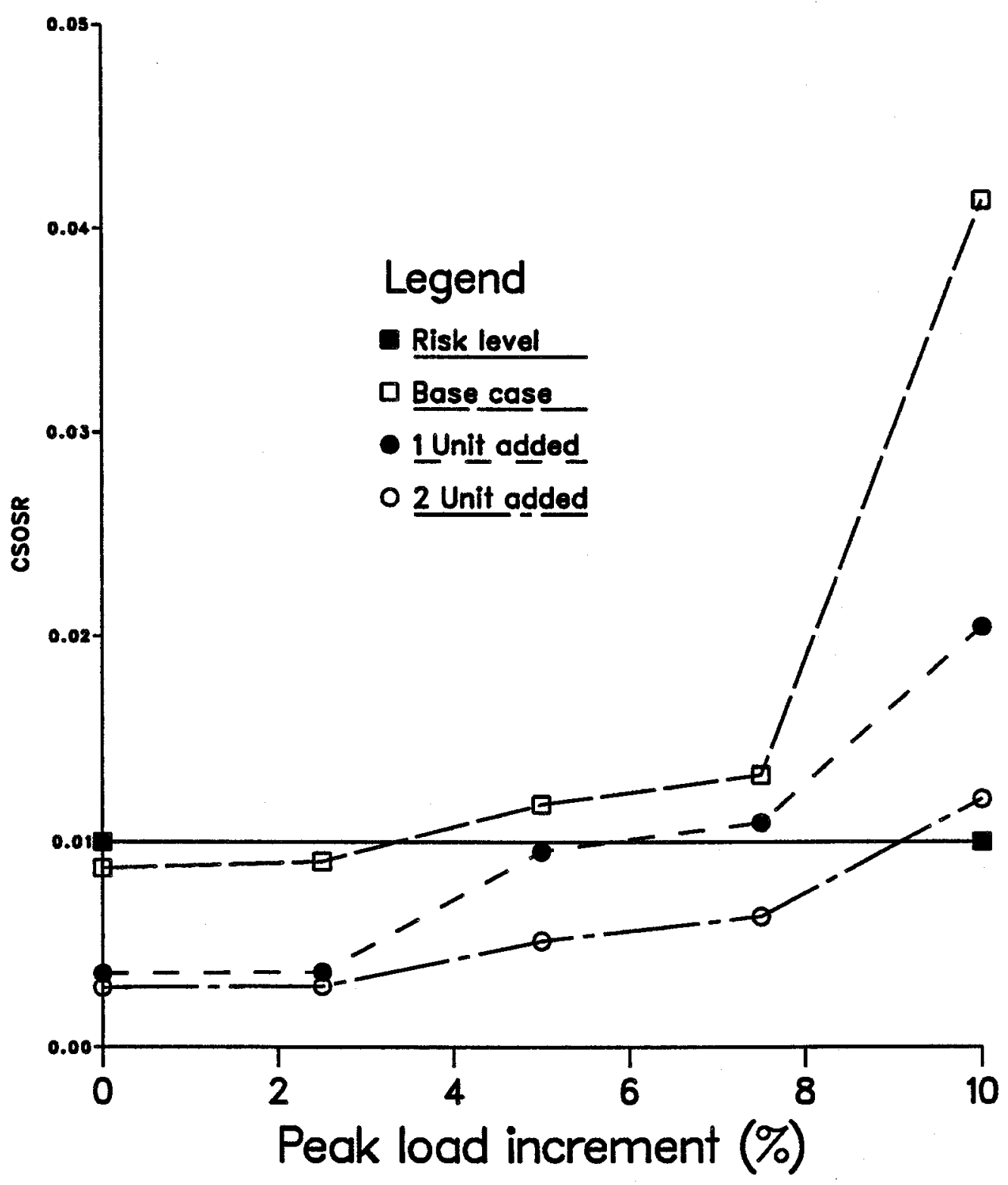

Figure 7.2: CSOSR variation with addition of generation at Bus 1 - MRBTS 


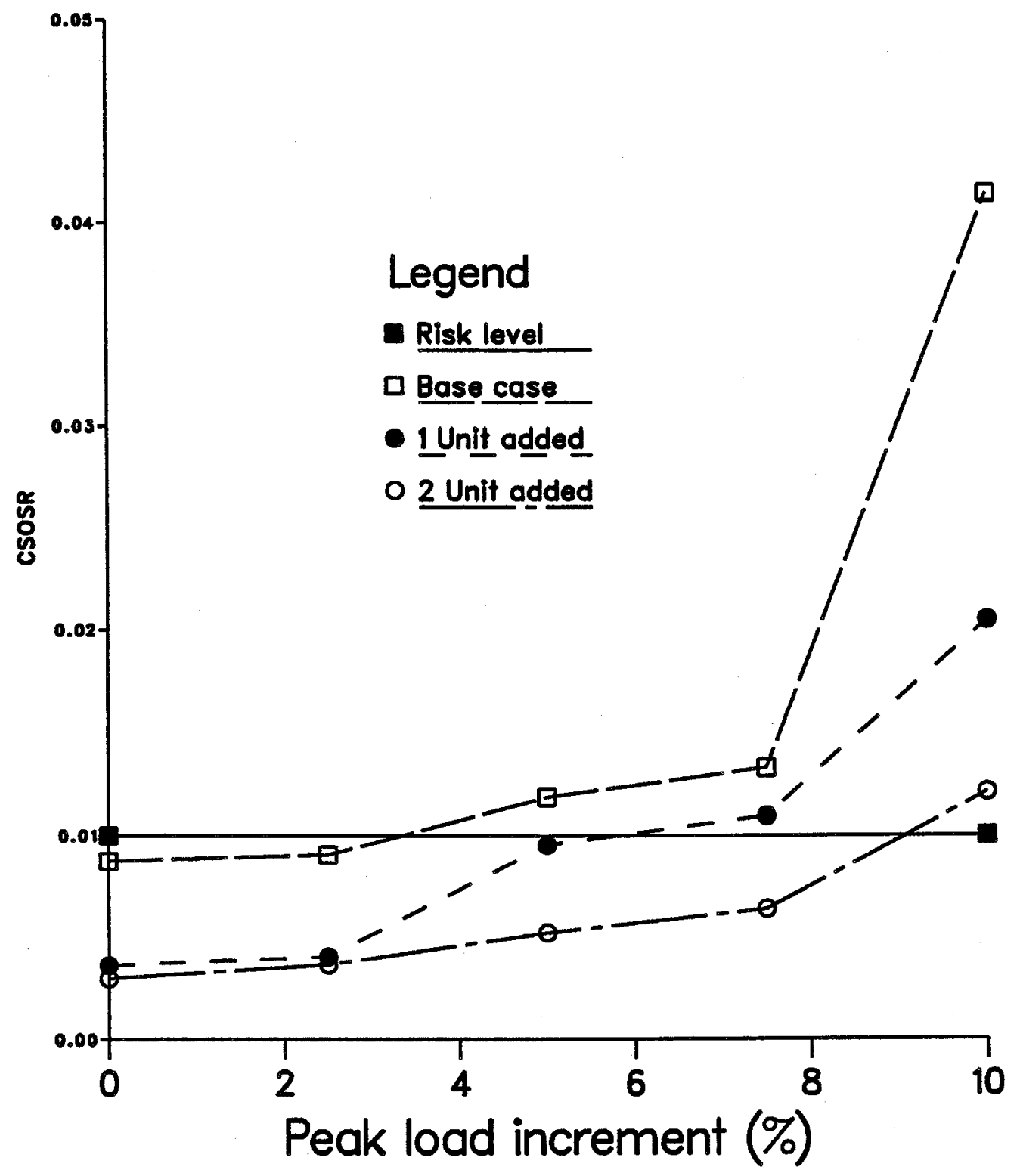

Figure 7.3: CSOSR variation with addition of generation at Bus 2 - MRBTS 
increase of up to 5\% in peak load can be served within the CSOSR level of 0.01 . Another generating unit addition is required when the peak load increment is approximately more than 5\%. The comparisons for additional units at bus 1 and bus 2 are shown in Figure 7.4. Another addition of two generating units is required at the peak load level increment of $10 \%$. The CSOSR is slightly lower in the case of adding generation facilities at bus 1 . Bus 1 , therefore, has been chosen as the location for the additional generating units in subsequent studies.

\subsubsection{Transmission Line Additions}

In the previous section, reduction in CSOSR was achieved by the addition of only generating units. It can be seen for this test system that due to capacity limitations of the transmission lines, some of the contingencies require load curtailment even when there is sufficient generation available. In this section, transmission line(s) are added to the system in order to demonstrate the effect of this addition on the CSOSR. In order to add transmission lines, it is assumed that lines can only be added to buses which have at least one line terminating at that bus in the MRBTS diagram shown in Figure 4.3. Two different approaches were employed to examine the effect on the CSOSR of transmission lines. These are

1. the addition of an extra transmission line at fixed line ratings and

2. variable transmission line ratings for the existing lines.

The first study involved the addition of extra lines to the base system. The line rating and other required data for the additional lines are assumed to be the same as the original line parallel to which the new line is connected. The CSOSR was evaluated for the new system as the load increases considering the generation data to be the same as that in the base case. The results are presented in Figures 7.5 and 7.6. The numerical values of CSOSR for different line additions are shown in Table 7.2. The results presented in these figures and table indicate that the added transmission lines can be ranked approximately as follows

1. line between buses 1 and 3

2. line between buses 2 and 4 


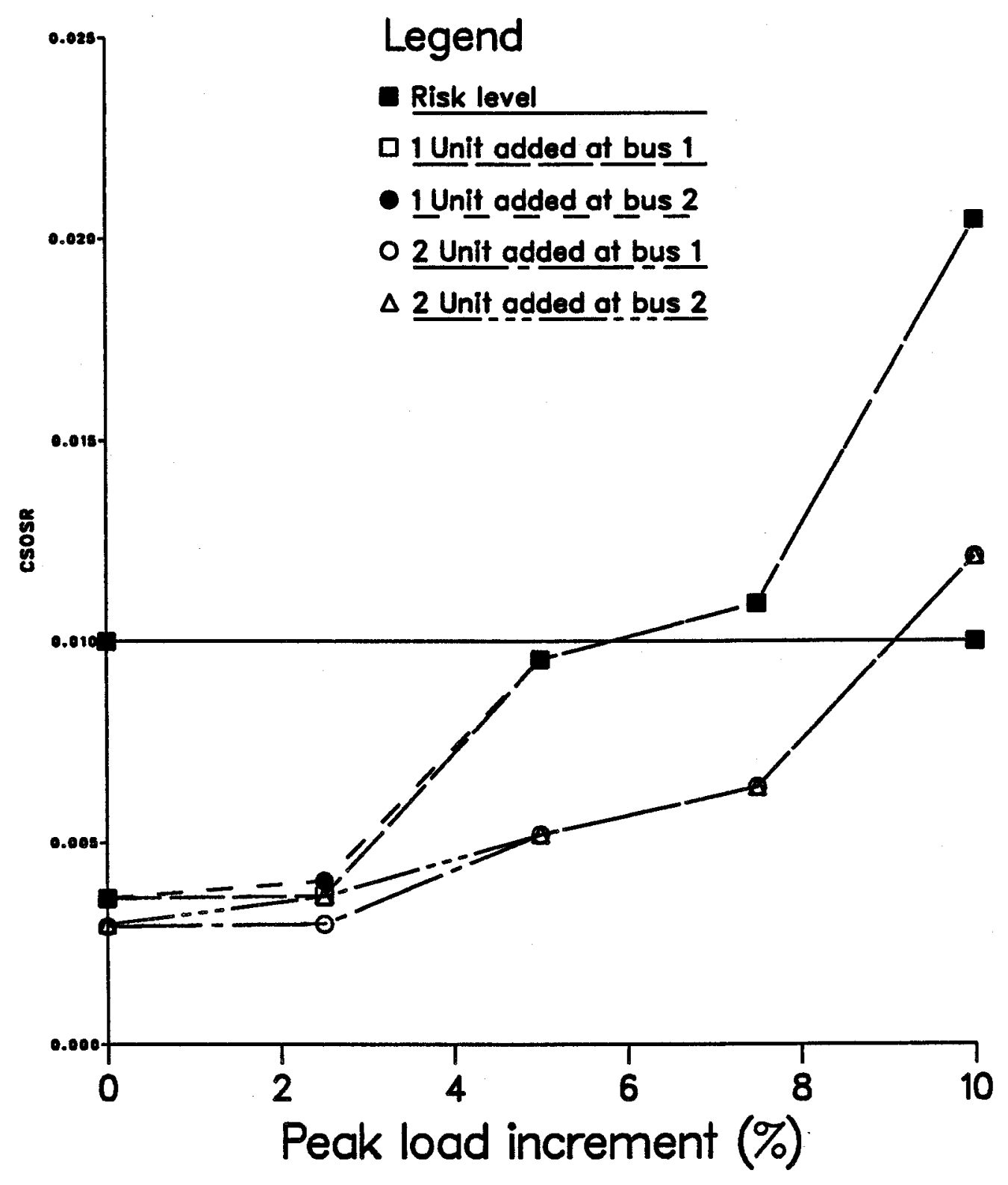

Figure 7.4: Comparison of addition of generation at Bus 1 and 2 - MRBTS 


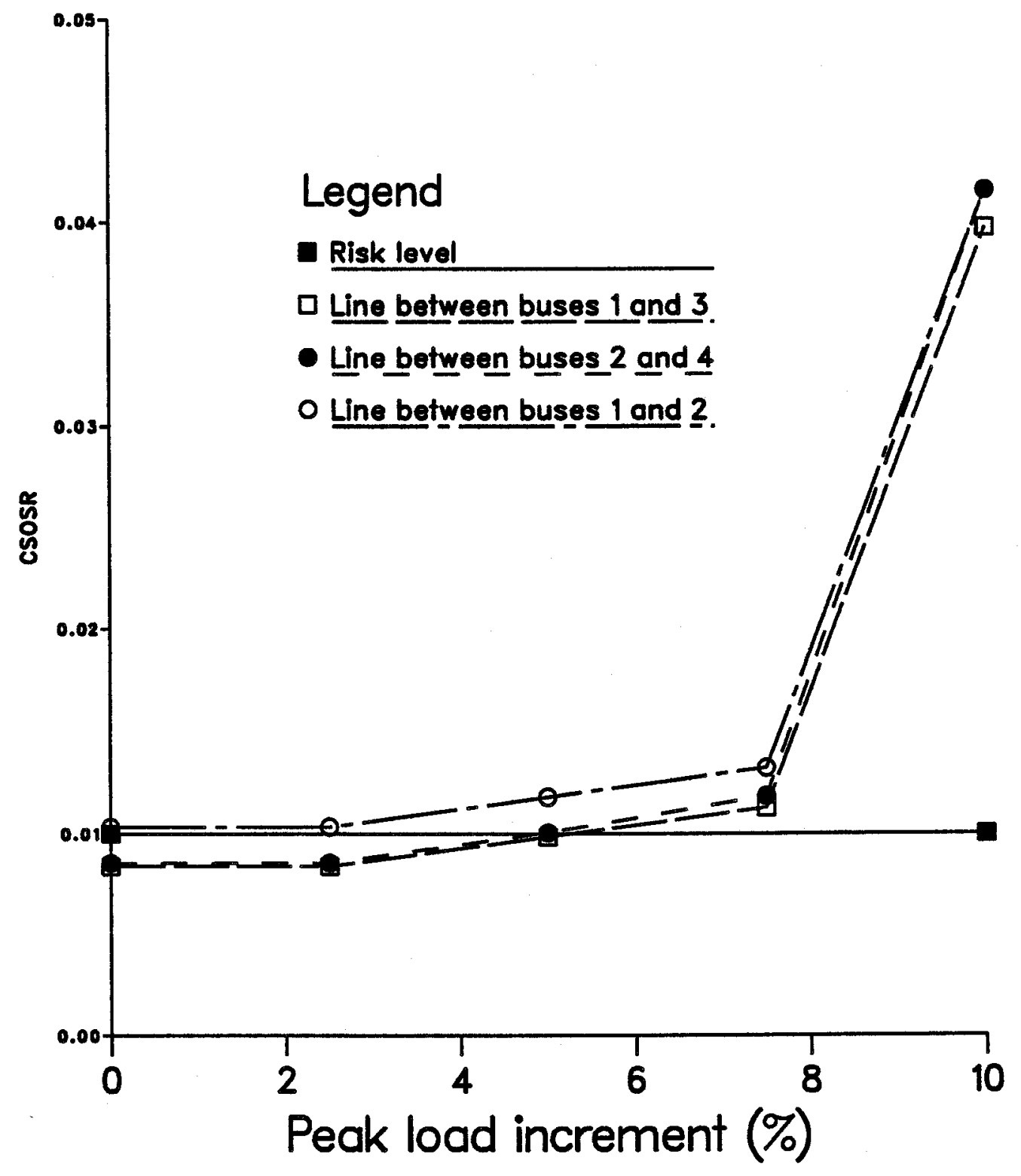

Figure 7.5: CSOSR variation with addition of transmission line - MRBTS 


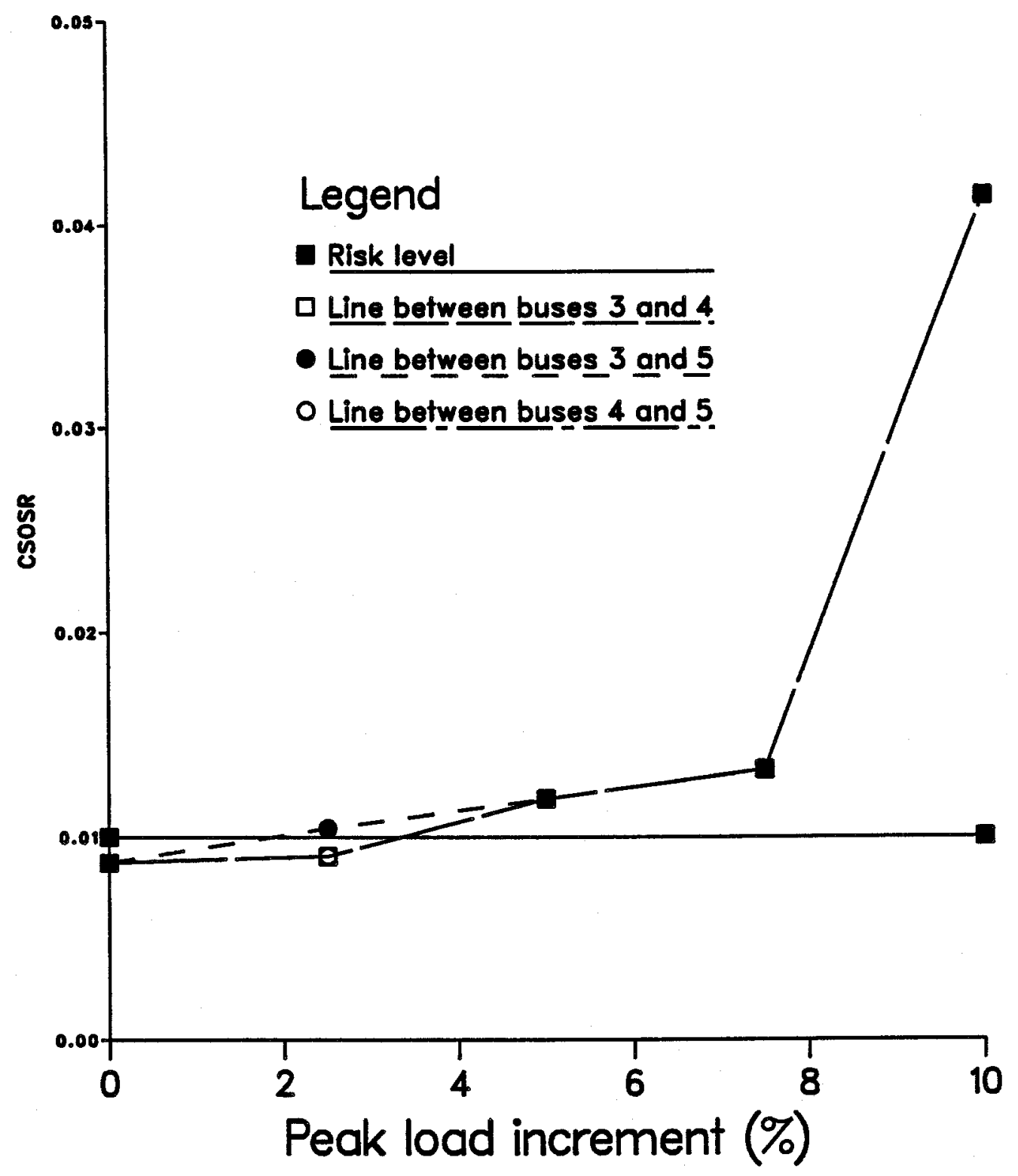

Figure 7.6: CSOSR variation with addition of transmission line - MRBTS 
3. line between buses 3 and 4

4. line between buses 1 and 2

5. line between buses 4 and 5

6. line between buses 3 and 5

Table 7.2: CSOSR for different line additions

\begin{tabular}{ccccccc}
\hline Line Added & $<---$ & \multicolumn{3}{c}{ CSOSR For Peak Load Increment Of } & -.--> \\
Between Buses & $0 \%$ & $2.5 \%$ & $5 \%$ & $7.5 \%$ & $10 \%$ \\
\hline & & & & & \\
\hline 1 and 3 & 0.008411 & 0.008411 & 0.009846 & 0.011266 & 0.039696 \\
2 and 4 & 0.008572 & 0.008578 & 0.010055 & 0.011831 & 0.041546 \\
1 and 2 & 0.010332 & 0.010338 & 0.011785 & 0.013205 & 0.041544 \\
3 and 4 & 0.008750 & 0.009053 & 0.011845 & 0.013261 & 0.041433 \\
3 and 5 & 0.008750 & 0.010415 & 0.011844 & 0.013260 & 0.041432 \\
4 and 5 & 0.008749 & 0.009052 & 0.011844 & 0.013260 & 0.041432 \\
\hline
\end{tabular}

This study indicates that the CSOSR can be reduced by the addition of a line in an appropriate location. The CSOSR increases from the base case values at a particular load level for some line additions. The CSOSRs for the base system configuration is 0.008751 and 0.009057 for load levels $0 \%$ and $2.5 \%$ increment respectively whereas the CSOSRs for the system with the addition of a line between buses 1 and 2 are 0.010332 and 0.010338 respectively for the same load level increments. The addition of a line between buses 1 and 3 , as the results indicate is the best choice among the six alternatives. Line addition between buses 1 and 3 is suitable up to a peak load increment of $5 \%$ and the line addition between buses 2 and 4 is suitable up to a peak load increment of approximately $4 \%$.

Line overload can lead to load curtailment if corrective action fails to alleviate the overload problem. Increasing the current ratings of the transmission lines may cause a reduction in overload problems and hence in the CSOSR level. A second study examined the effect on the CSOSR of varying the line ratings. The base configuration of the MRBTS i.e. without any extra generating units and transmission lines was utilized in this 


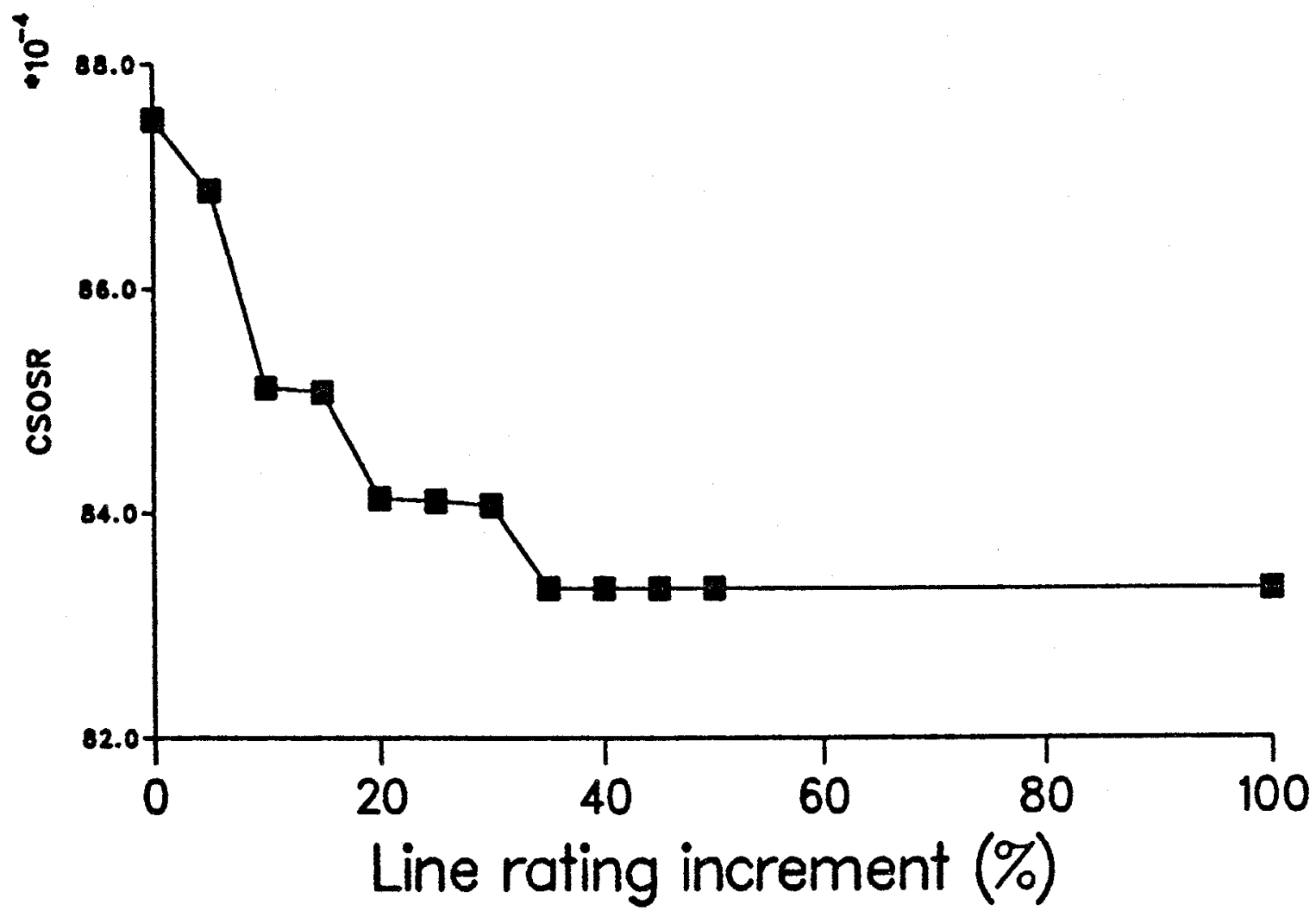

Figure 7.7: CSOSR variation with transmission line rating - MRBTS

study. The results for a fixed system peak load of $185 \mathrm{MW}$ are shown in Figure 7.7. This figure indicates that the CSOSR does not vary significantly as the line ratings increase and for a line rating of $35 \%$ or more the CSOSR is constant. A further study was performed in which the line ratings of the original MRBTS were fixed at 1.35 times their base values. The generation data was as in the base case. Figure 7.8 shows the variation in CSOSR with load increment. These results can be compared with those of Figures 7.2 and 7.3 and can be used in an assessment of the benefits associated with increasing the power flow ratings of the transmission lines.

\subsubsection{Composite Generation And Transmission System Reinforcement}

The addition of either generating units or transmission lines to the MRBTS reduces the CSOSR level of the entire system as shown earlier. The addition of only generating units to the existing system can result in a situation where the additional units may not lead to a significant decrease in the CSOSR of the system because the existing transmission lines may not be capable of transporting the generated energy to the major 


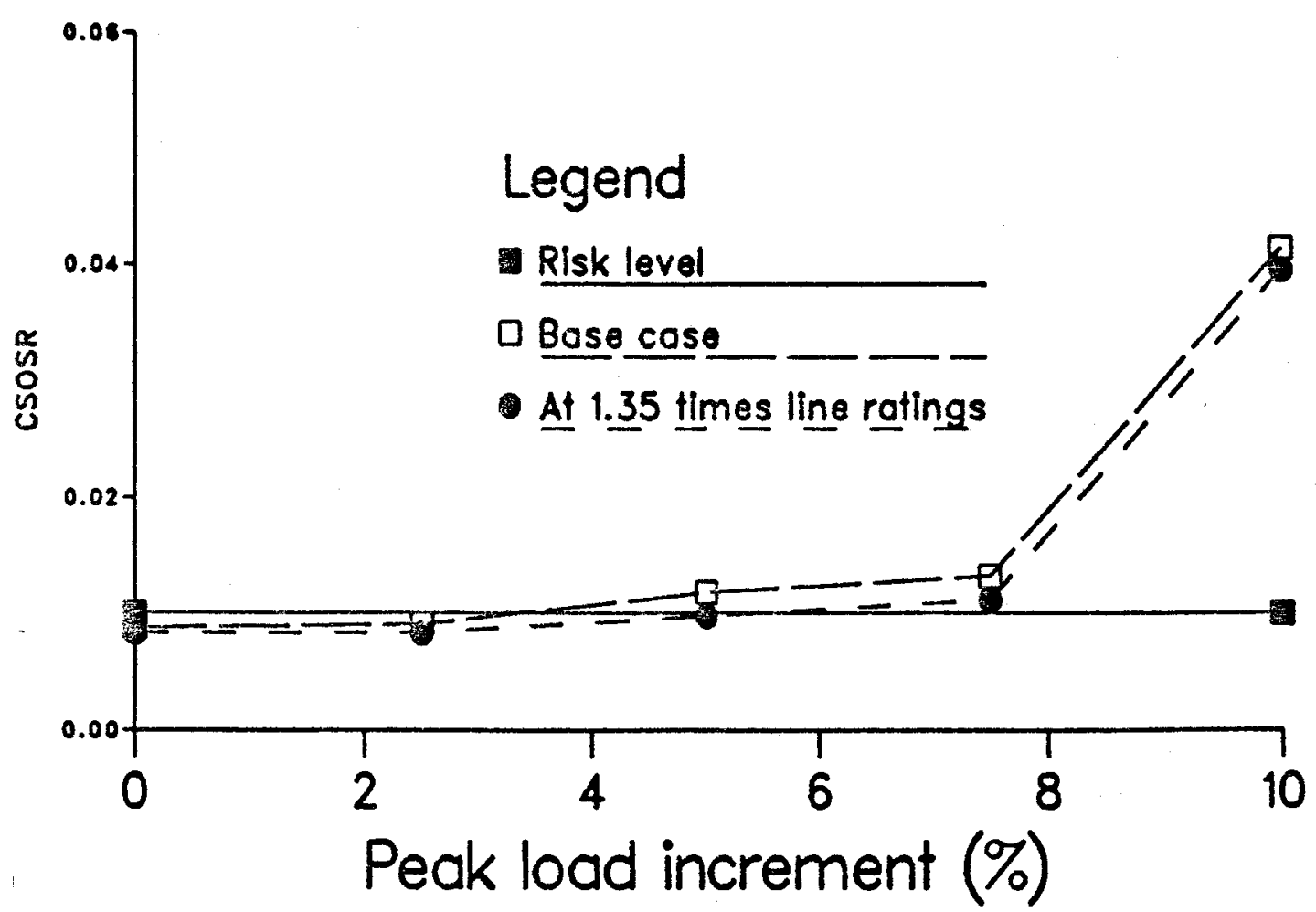

Figure 7.8: CSOSR variation with load increment at 1.35 times of transmission line rating - MRBTS

load points. The addition of only transmission lines to the existing system may also not be a good planning decision if the existing lines can carry easily the generated energy from the generator buses to the major load points. The objective of this example is to illustrate the addition of both generating units and transmission facilities concurrently to the system so that none of the situations noted earlier occurs. The addition of facilities may be done by considering

1. economy or

2. reliability or

3. both economy and reliability.

The decision to add new lines or generators, therefore, depends on the planner's viewpoint and priorities. Since this research work deals with the development of reliability methodology, therefore, reliability of the system will be considered to be the most important issue. 
As noted earlier, as the load grows the addition of generation at bus 1 or the addition of a line between buses 1 and 3 for the MRBTS are the best choices among the considered alternatives. A comparison of these two additions to the base MRBTS is shown in Table 7.3. It can be seen from Table 7.3 that one line addition and one generator unit addition is capable of carrying up to $5 \%$ load increment for the MRBTS within the specified CSOSR level. The improvement in CSOSR is $19.39 \%$ in the case of

Table 7.3: Comparison of facilities addition

\begin{tabular}{|c|c|c|c|c|c|}
\hline $\begin{array}{l}\text { Facility } \\
\text { Addition }\end{array}$ & <\% & $\begin{array}{c}\text { CSOSR For } \\
2.5 \%\end{array}$ & $\begin{array}{l}\text { Peak Load } \\
5 \%\end{array}$ & $\begin{array}{c}\text { ncrement of } \\
7.5 \%\end{array}$ & $--->>$ \\
\hline \multirow{3}{*}{$\begin{array}{l}\text { Base case } \\
\text { One line between } \\
\text { buses } 1 \text { and } 3 \\
\text { One generator } \\
\text { at bus } 1\end{array}$} & 0.008751 & 0.009057 & 0.011843 & 0.013260 & 0.041384 \\
\hline & 0.008411 & 0.008411 & 0.009846 & 0.011266 & 0.039696 \\
\hline & 0.003618 & 0.004036 & 0.009546 & 0.010939 & 0.020460 \\
\hline
\end{tabular}

generator addition at bus 1 compared to $16.86 \%$ for the addition of a line between buses 1 and 3. The improvement in CSOSR is calculated by

\% CSOSR improvement $=\frac{\text { New CSOSR }- \text { Base Case CSOSR }}{\text { Base Case CSOSR }} \times 100 \%$

Assume that a $10 \mathrm{MW}$ generating unit is added to bus 1 and the peak load level is increased by $5 \%$. This is considered to be the base configuration and is examined in regard to additional required facilities with increased system load, at a CSOSR level of 0.01 .

The additional generating unit at bus 1 is capable of carrying a peak load increment of below $7.5 \%$. At this level, another generating unit is required at bus 1 . At $10 \%$ or more load increment, the CSOSR again exceeds the specified risk level and additional generating units are required to bring the CSOSR within the specified CSOSR level of 0.01. It is also possible to bring the CSOSR below the limit by adding one line at this 
particular load level. Therefore one transmission line was added to the new base system at this load level $(+10 \%)$. Based on the study where only a transmission line is added, a new line between buses 1 and 3 is the best option in terms of CSOSR level reduction. The new line was, therefore, added between these buses at the $10 \%$ peak load increment and the corresponding CSOSR calculated. As the load continues to increase, further additions can be considered in order to keep the CSOSR below the specified level. At the $12.5 \%$ peak load level increment, another generating unit was added at bus 1 and the CSOSR evaluated. Another one generatng unit at bus 2 and one line between buses 2 and 4 were added at the $15 \%$ peak load level increment. The results associated with this particular expansion scheme are shown in Figure 7.9. It can be seen that composite generation and transmission facilities reinforcement can be used to maintain the required CSOSR level for the MRBTS. This procedure can be used to assess any particular pattern of generating unit and/or transmission line reinforcement for a given system.

\subsection{EXPANSION PLANNING IN THE IEEE-RTS}

This section of the chapter applies the CSOSR index defined earlier to generation and transmission expansion planning in the IEEE-RTS. The base case generation and transmission system data are provided in Appendix B. It can be seen from an HLI study that the generation system for the IEEE-RTS is unreliable and has an LOLE of 9.39418 hours/year and an LOEE of $1176 \mathrm{MWh} /$ year when the system peak load is $2850 \mathrm{MW}$ [107]. The CSOSR of this system is 0.10285 for a peak load of $2850 \mathrm{MW}$. In order to provide some ability for load growth in the existing system and a datum for expansion, a CSOSR of 0.17 has been considered as the specified acceptable risk level.

\subsubsection{Generation Capacity Addition}

The purpose of this study is to demonstrate how the CSOSR can be utilized to justify the addition of new generating units to the system as the system peak load increases with time. The additional generating unit data used in these studies are provided in Table 7.4 [107]. In this test system there are 32 generating units connected to 10 different PV buses. The location of generating unit additions is an important factor. There are, therefore, many alternatives that could be considered for these studies. The buses in the system can be classified as follows: 


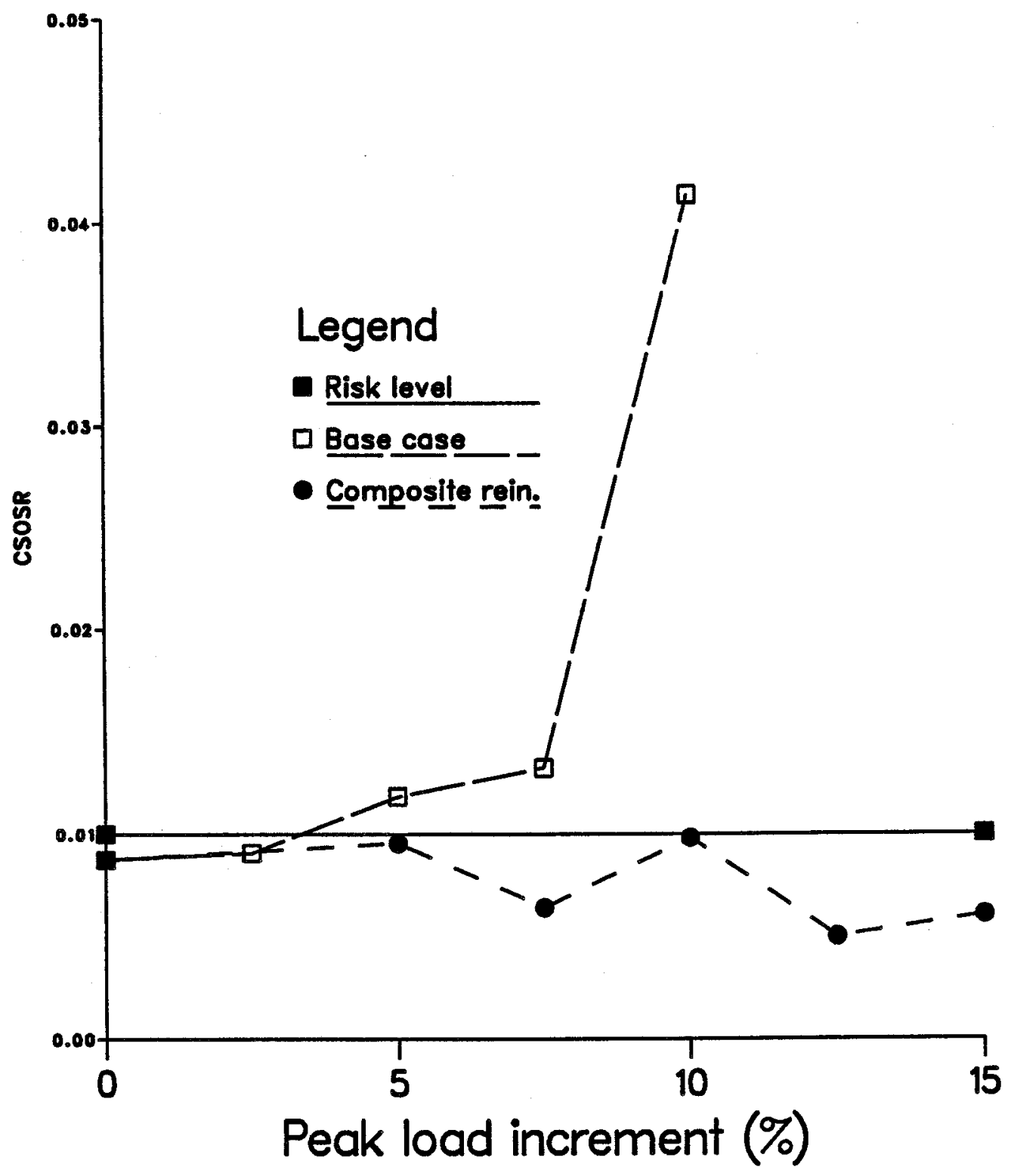

Figure 7.9: CSOSR variation for composite system - MRBTS 
Table 7.4: Additional generating units for the IEEE-RTS

\begin{tabular}{cccc}
\hline $\begin{array}{c}\text { Capacity } \\
(\mathrm{MW})\end{array}$ & FOR & $\begin{array}{c}\text { MTTF } \\
\text { (Hours) }\end{array}$ & $\begin{array}{c}\text { MTTR } \\
\text { (Hours) }\end{array}$ \\
\hline 25 & 0.12 & 550 & 75 \\
\hline
\end{tabular}

1. south region $138 \mathrm{KV}$ buses: buses 1 to 10 and

2. north region $230 \mathrm{KV}$ buses: buses 11 to 24 .

The total generation available in the south region is $684 \mathrm{MW}$ and that in the north region is $2721 \mathrm{MW}$. The load connected in the south region is $1332 \mathrm{MW}$ and in the north region is $1518 \mathrm{MW}$ at the system peak of $2850 \mathrm{MW}$. Generation can be added to any generator bus(es) in the two regions. Generation expansion is therefore limited by considering addition at three buses in the north region and three buses in the south region. It is assumed that the generating unit(s) could be connected to the following buses

\section{1. bus 1 \\ 2. bus 2 \\ 3. bus 7 \\ 4. bus 15 \\ 5. bus 16 \\ 6. bus 23}

The results for the addition of generating units at different buses are shown in Figures 7.10 to 7.15. The CSOSR reduces as generation is added to the system, but the reduction in the CSOSR at higher load levels for each additional generating unit is small compared to the case of MRBTS, as can be seen from these figures. The reason for this is that a $1 \%$ load increment for this test system is equal to $28.5 \mathrm{MW}$ whereas each additional generating unit that is being added to the system has a capacity of $25.0 \mathrm{MW}$. 


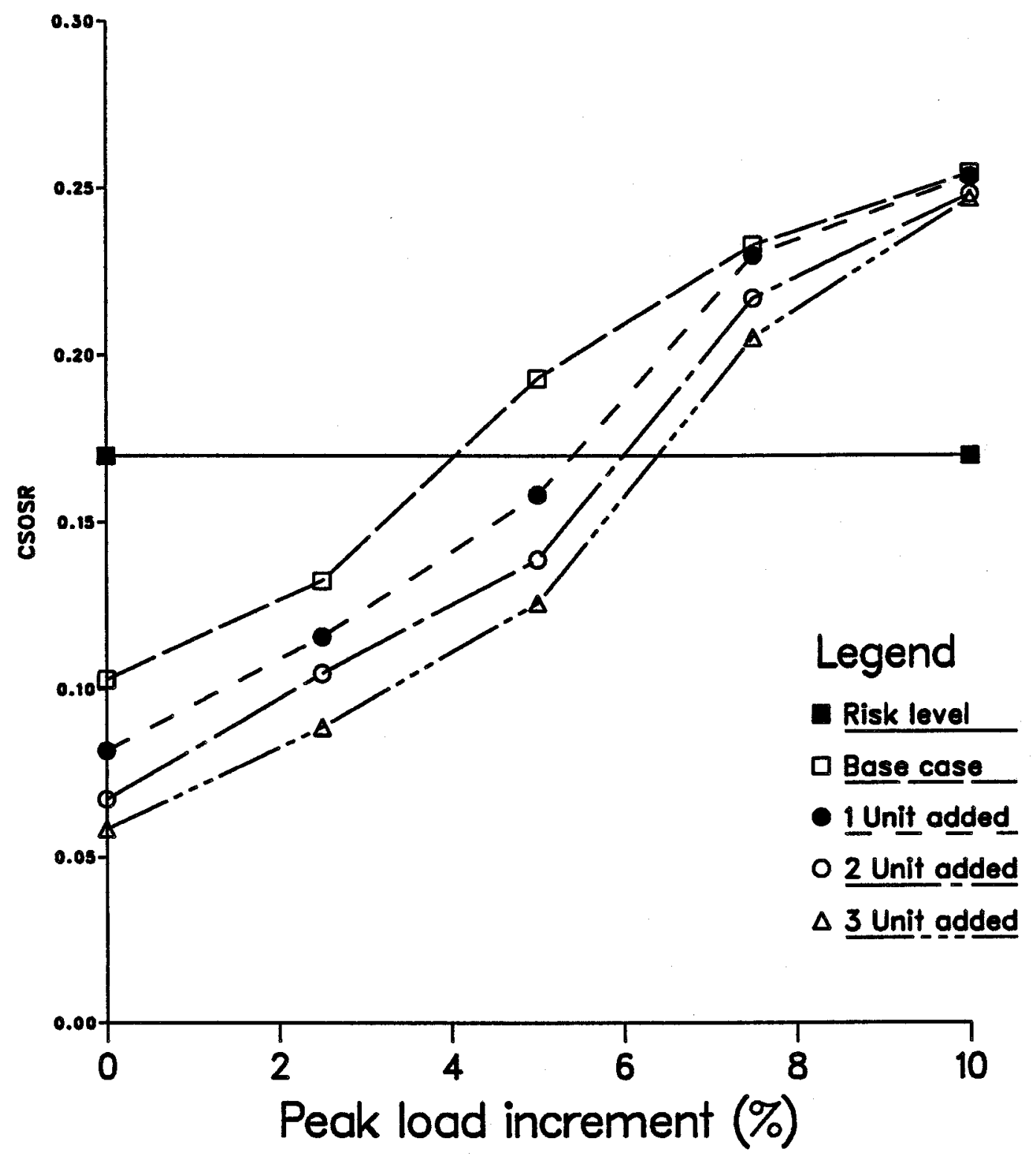

Figure 7.10: CSOSR variation with addition of generation at Bus 1 - IEEE-RTS 


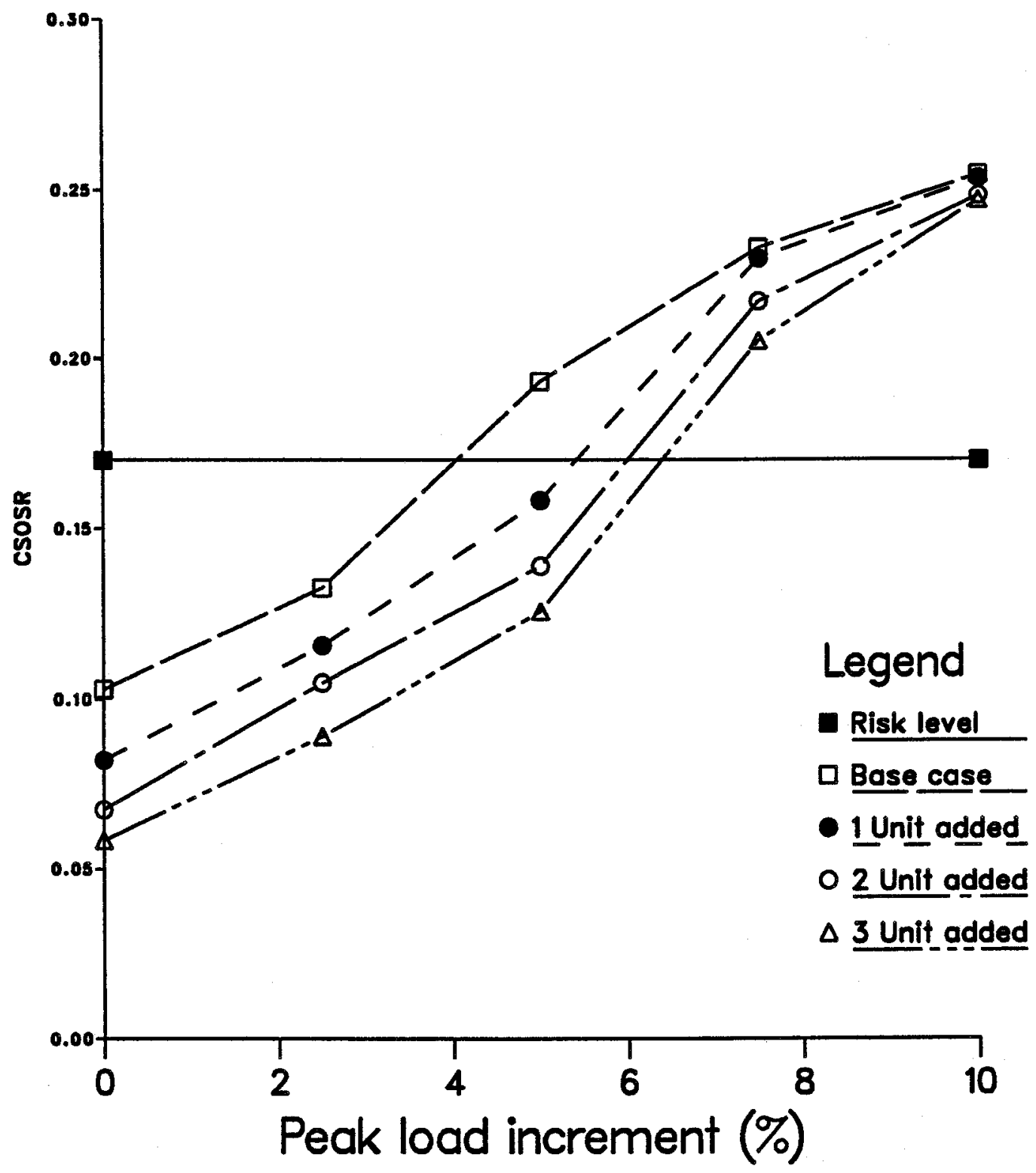

Figure 7.11: CSOSR variation with addition of generation at Bus 2 - IEEE-RTS 


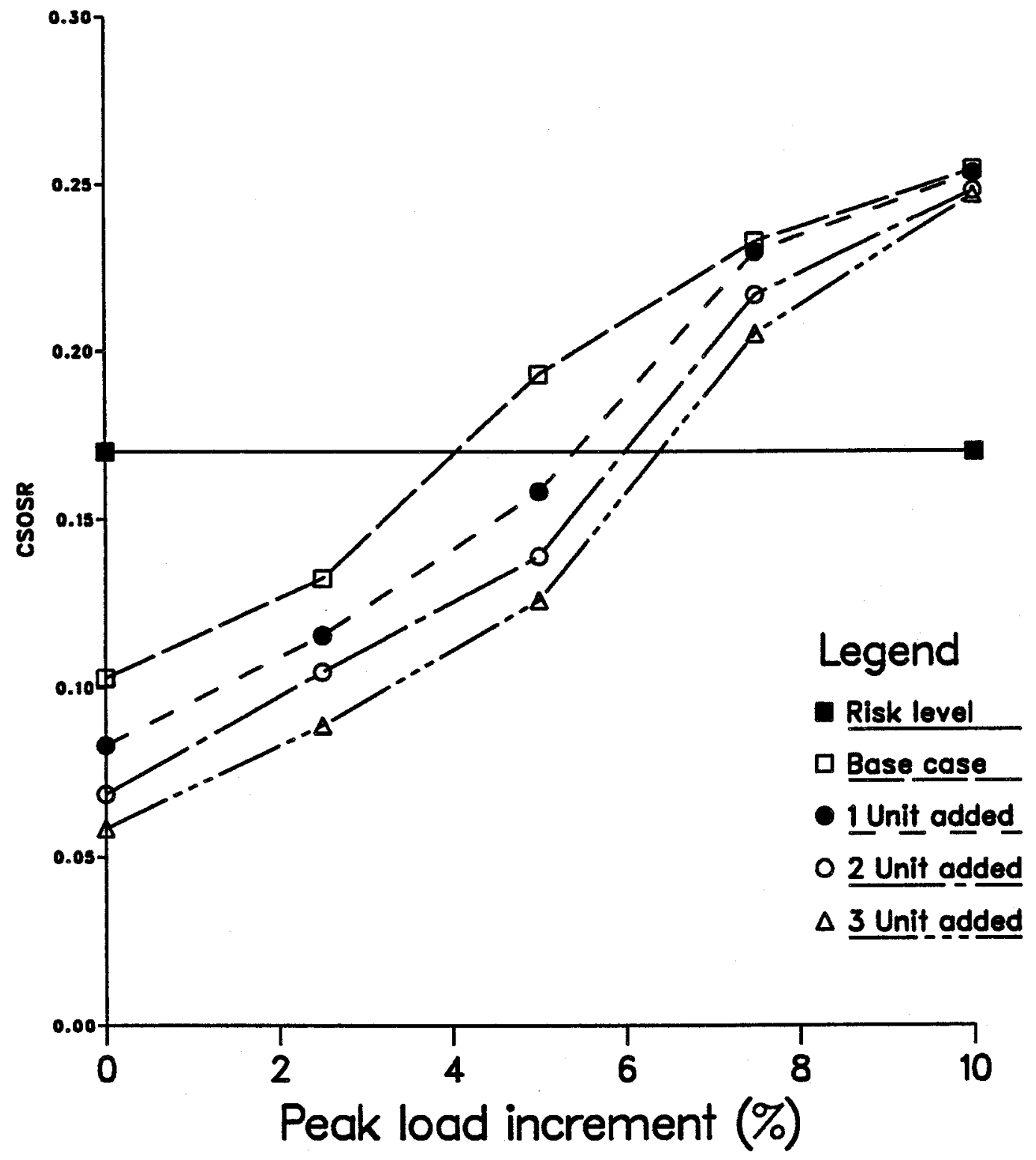

Figure 7.12: CSOSR variation with addition of generation at Bus 7 - IEEE-RTS 


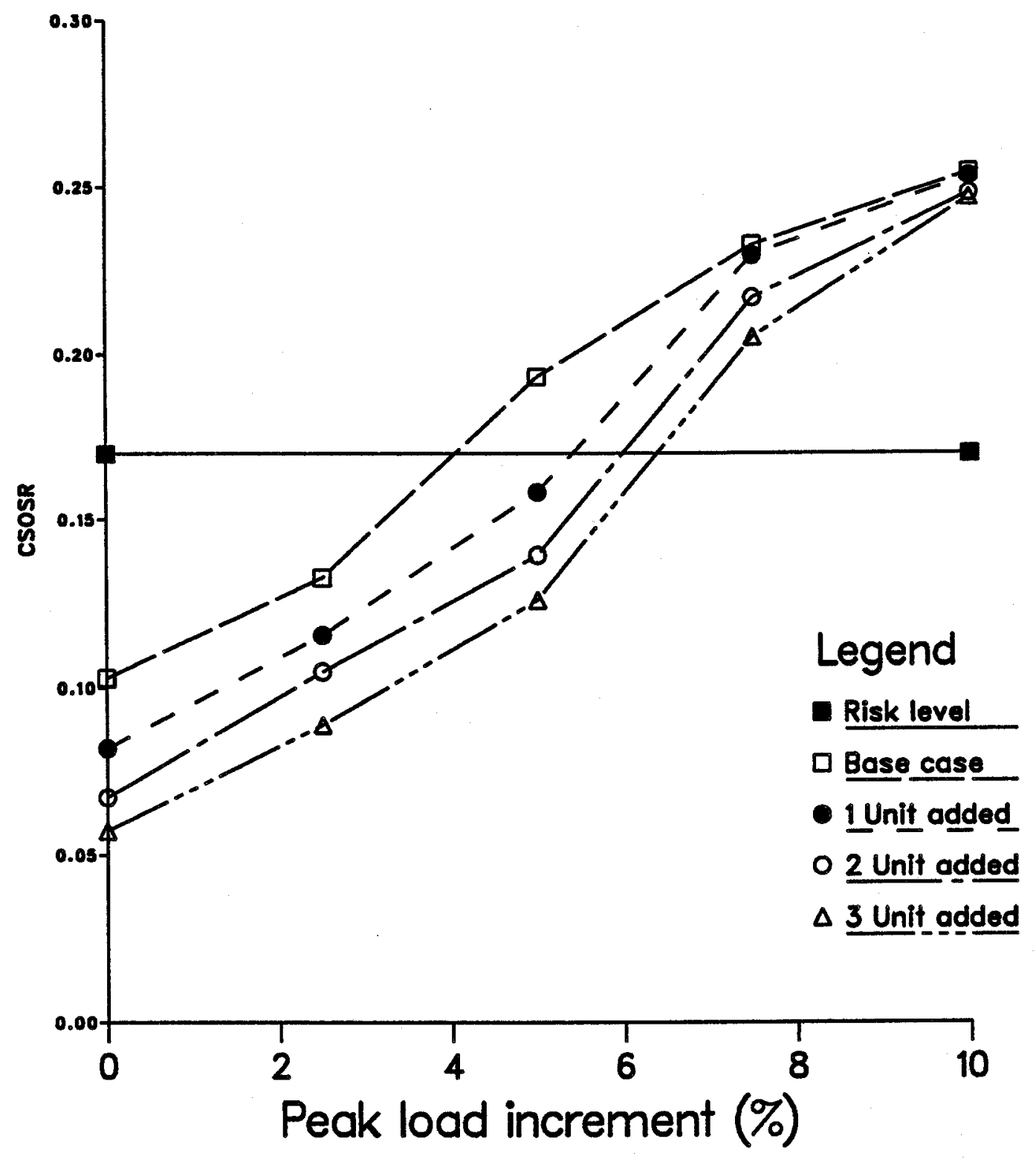

Figure 7.13: CSOSR variation with addition of generation at Bus 15 - IEEE-RTS 


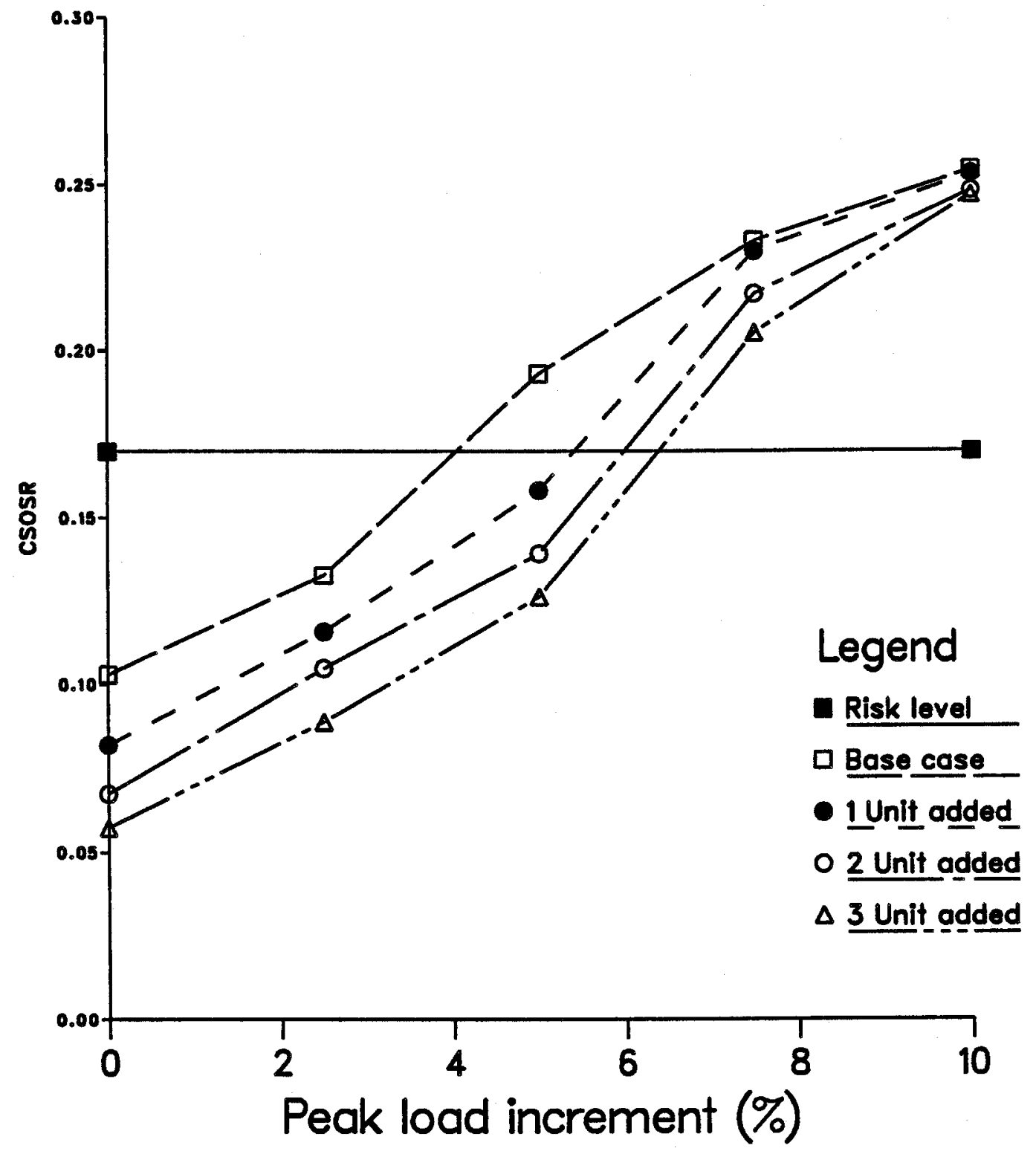

Figure 7.14: CSOSR variation with addition of generation at Bus 16 - IEEE-RTS 


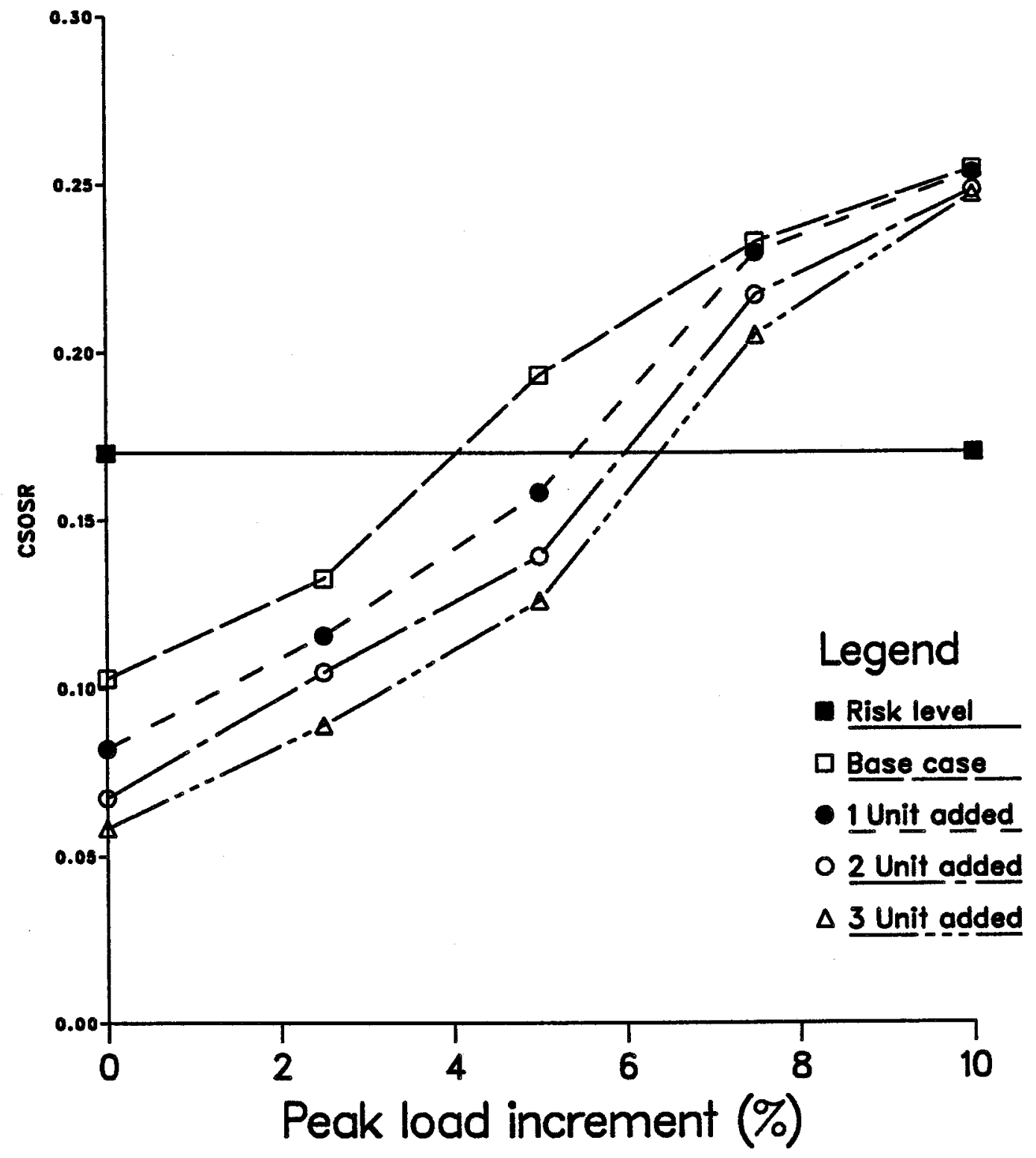

Figure 7.15: CSOSR variation with addition of generation at Bus 23 - IEEE-RTS 
The capacity of each generation addition is lower than the load increment and therefore has a relatively lower impact on the CSOSR compared to the MRBTS case. Additional generation is required at the $4 \%$ load increment for the assumed acceptable CSOSR level of 0.17 . This additional generation when added at bus 1 , can carry a load level of up to 5.5\% load increment. An additional generator was therefore added at the 5\% load increment and the augmented system was found to be capable of carrying a peak load increment of up to $6 \%$. At the $6 \%$ load increment, additional generating units have to be added to bring the CSOSR below the acceptable limit.

$$
\text { Hows? }
$$

It can be seen from Figures 7.10 to 7.15 that the addition of generation at different buses creates only slightly different effects on the CSOSR. This is due to the relatively oversized transmission facilities in the IEEE-RTS. This point is illustrated in the next section. Addition of generation at bus 1 results in a lower CSOSR than that obtained in the other five cases considered. Therefore, generation additions at bus 1 are utilized for subsequent composite system reinforcement.

\subsubsection{Transmission Line Additions}

The CSOSR decreases as generation is added to the system. The addition of only generating units to the existing system may not be sufficient to avoid load curtailment(s) due to transmission system deficiencies. The effect of considering only transmission line additions to the existing system is illustrated in this section. The IEEE-RTS is a comparatively large system as noted earlier and has 38 transmission lines in the basic configuration. There are many alternatives that could be considered in these studies. In order to limit the number of cases to be investigated and to show the effect on the risk index of transmission line addition(s), it has been assumed that the new transmission lines are connected as follows:

1. between buses 1 and 3 ,

2 . between buses 2 and 6 ,

3. between buses 13 and 23 and

4. between buses 15 and 24 .

In the above four cases, two lines are in the south and the remaining two are in the north 
regions. The parameters for a new line are identical to the existing line parallel to which the line is connected. The risk indices for the above transmission line additions to the existing system are shown in Figures 7.16 and 7.17.

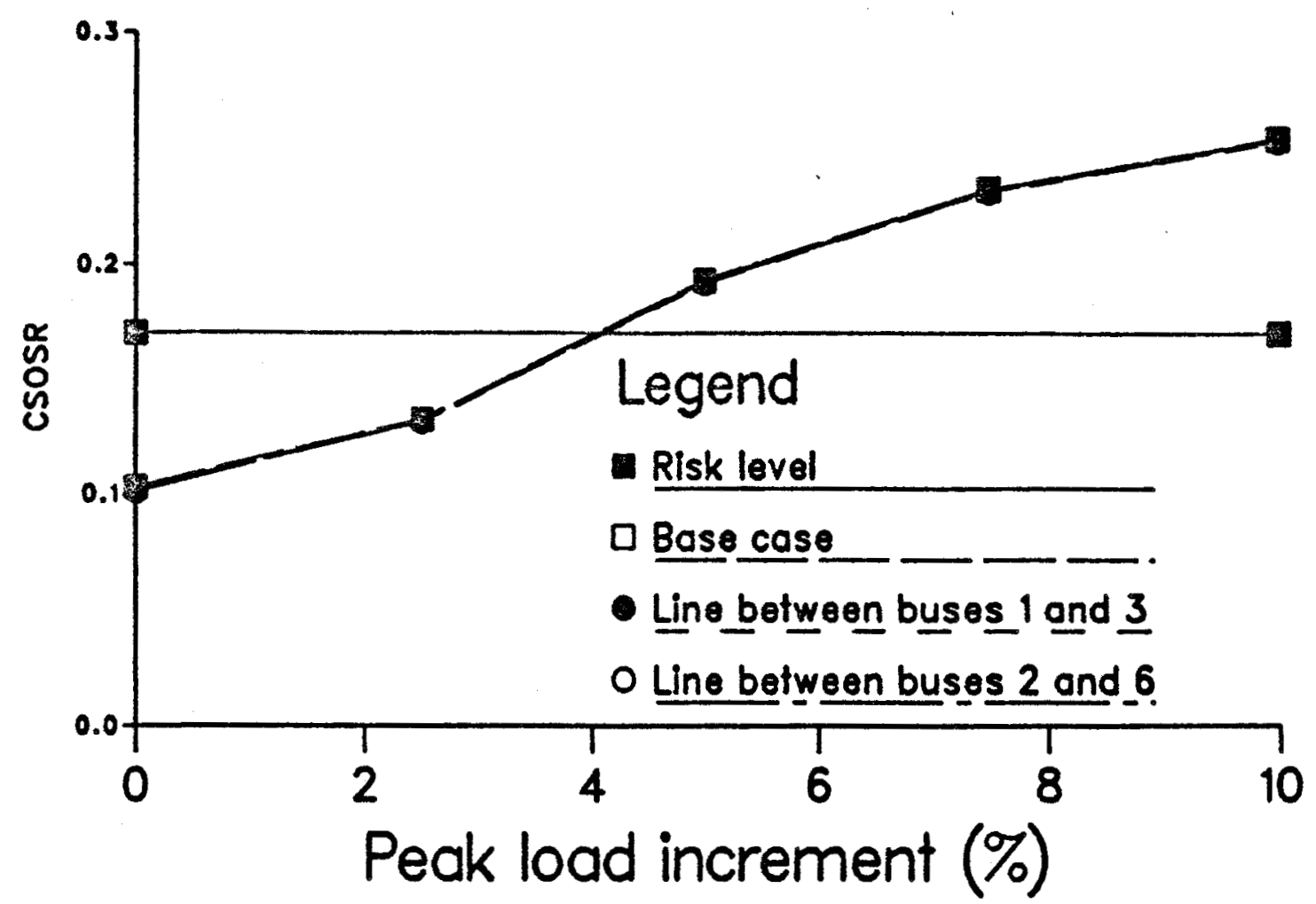

Figure 7.16: CSOSR variation with addition of transmission line in the south region - IEEE-RTS

It can be seen from these figures that the transmission line additions have very little effect on the CSOSR. The reason for this is that the IEEE-RTS has an over sized transmission system and the base system transmission is quite sufficient to carry the increased load demand. The risk index increases beyond the acceptable CSOSR level mainly due to the generation system unreliability. Therefore, the additional transmission line(s) have a very small effect on the CSOSR for the peak load increments shown in the figures. Further study was performed by varying the line ratings for the fixed peak load level of $2850 \mathrm{MW}$. The variation of the CSOSR with line rating increment is shown in Figure 7.18. It can be seen from this figure that the CSOSR does not vary with the line rating which also indicates that the IEEE-RTS has an over sized transmission system. 


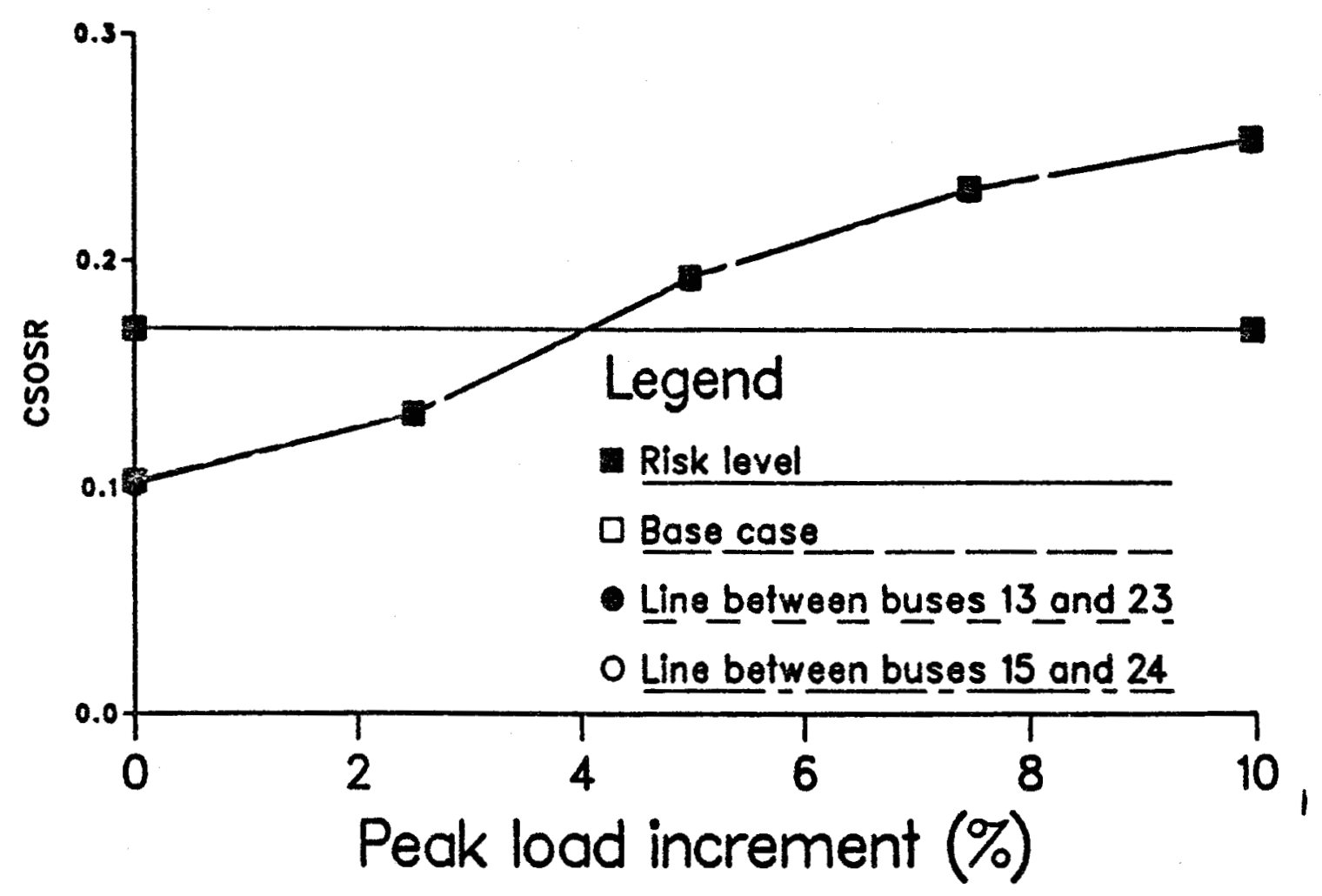

Figure 7.17: CSOSR variation with addition of transmission line in the north region - IEEE-RTS

\subsubsection{Composite Generation And Transmission System Reinforcement}

The addition of only generating units or transmission lines may not be sufficient to provide an acceptable risk in a composite generation and transmission system and the simultaneous addition of both facilities may be required. The effect of on the CSOSR of adding both generating units and transmission lines for increasing system peak load is examined in this section. As noted earlier, the IEEE-RTS is a relatively large system and many alternatives can be considered in this study. It is shown in an earlier section that adding generating units at bus 1 is more beneficial than adding them at other points and the location of additional transmission lines is not critical. It has therefore been assumed that all the additional generating units are located at bus 1 and the new transmission lines are connected as follows:

1. line between buses 1 and 3 and

2. line between buses 2 and 4. 


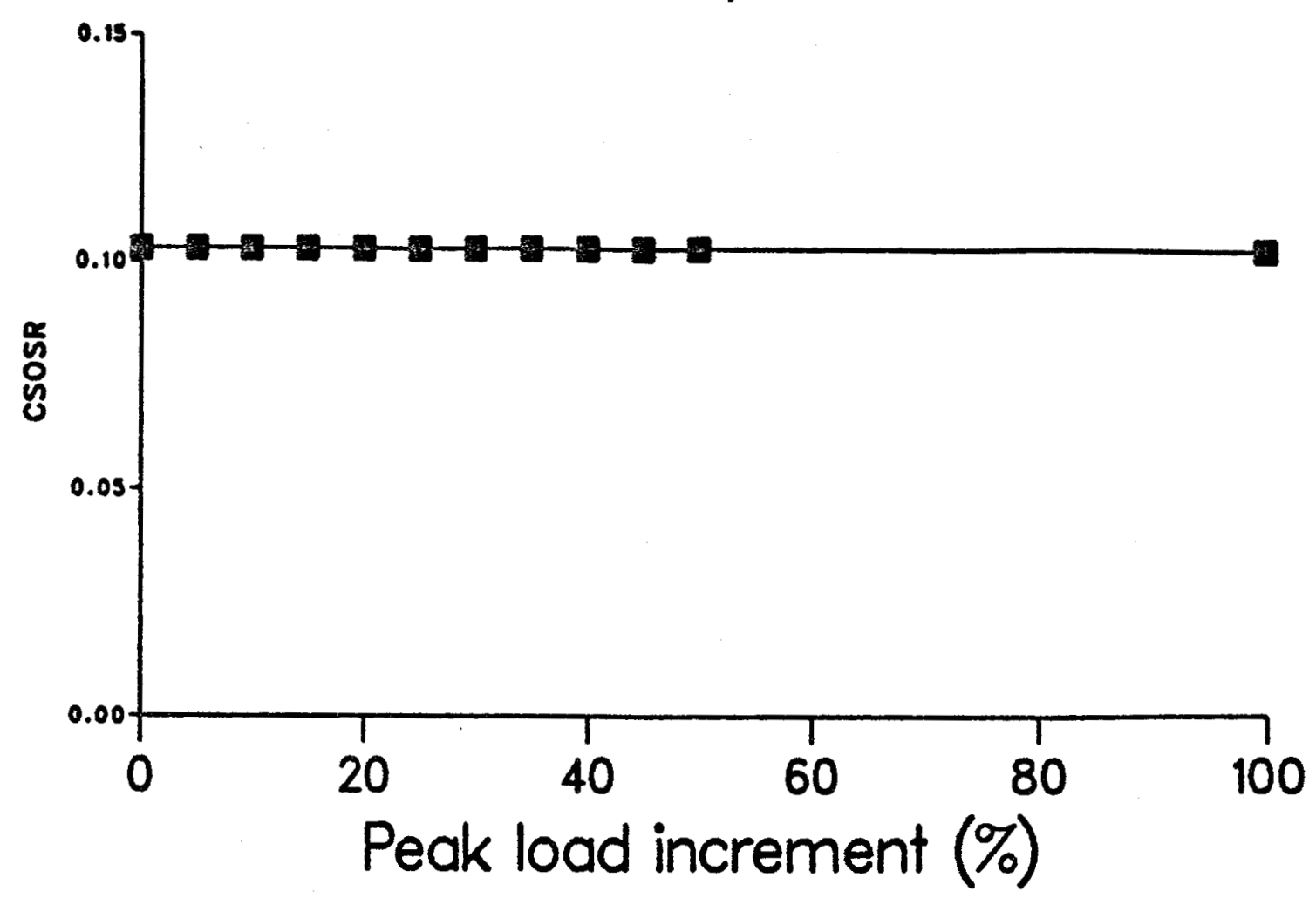

Figure 7.18: CSOSR variation with transmission line rating - IEEE-RTS

The additional generating units added are given in Table 7.4. The additional line has the same parameters as the existing line parallel to which the new line is connected. An additional generating unit and one transmission line between buses 1 and 3 were added to the system at the $4 \%$ peak load increment. An additional generating unit and the line between buses 2 and 4 were then added to the system at the 5\% peak load increment. Additional generating units were then added at the $6 \%$ and $7 \%$ peak load increments. The results of this study are shown in Figure 7.19. This figure indicates that the effects of simultaneous generation and transmission reinforcement to the existing system are not significantly different from those obtained by considering only the addition of generating units in the IEEE-RTS for the load levels considered. This would obviously not be the case if the load increases to the point at which the transmission system is no longer oversized. 


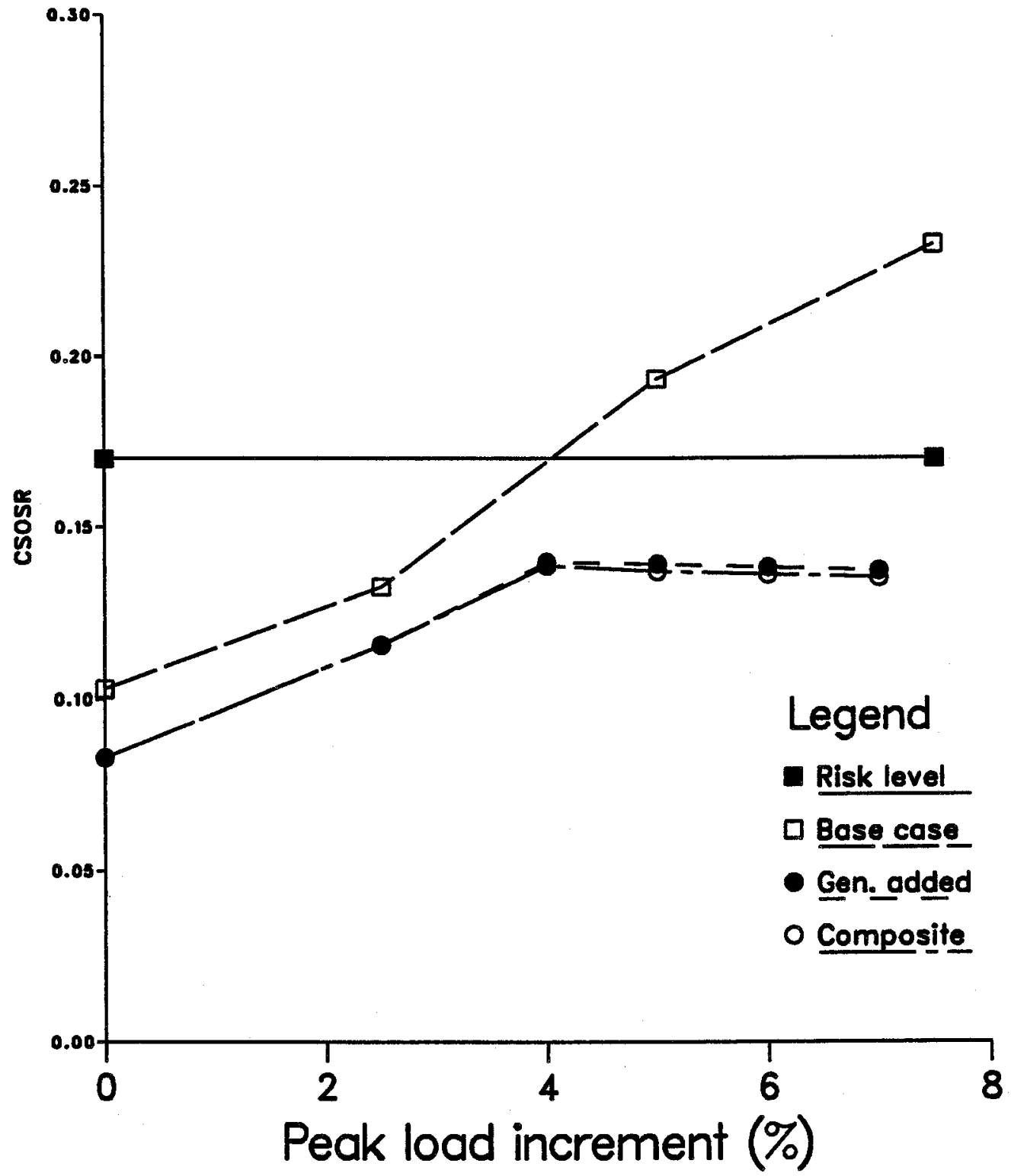

Figure 7.19: CSOSR variation for composite system - IEEE-RTS 


\subsection{SUMMARY}

The Composite System Operating State Risk (CSOSR) has been defined in this chapter as the probability of residing in an undesirable system operating state. The utilization of this risk index in simple system expansion planning is illustrated using the two test systems. The actual location of a generating unit addition is not recognized in a conventional HLI study. The location of additional generating unit, however, as well as additional transmission lines is very important in an HLII assessment. It has been found that the addition of facilities at some locations may make the system marginally more unreliable than the existing base system. In the case of the MRBTS, for example, the risk index with the addition of a line between buses 1 and 2 is greater than that of the base configuration up to a certain peak load increment. Composite reinforcement with both generation and transmission gives better results than the simple addition of either generation or transmission facility for the MRBTS.

The variation in the CSOSR index with additional generation in the IEEE-RTS is not as significant as compared to the MRBTS case. Transmission facility reinforcements have very little effect on the risk index for this test system due to the fact that the IEEERTS has an over sized transmission system. Composite reinforcement for the IEEE-RTS is therefore only slightly better than the generation addition case.

The studies shown in this chapter are intended to illustrate the utilization of the new CSOSR index. The expansion example considered are simplistic and there are many other considerations which must included in an actual expansion planning study.

The system load does not stay at its peak load throughout a given period of time e.g. a day or a year. All the system generating units are therefore not placed in service all of the time and are usually committed to the system at a particular load level in order to satisfy a desired risk level known as the 'operating risk'. The number of units to be committed and the corresponding operating risk depends mainly on the lead times of the different generating units. The effects of this lead time and the number of generating units committed to the system on the probabilities of the different operating states are considered in the next chapter. 


\section{SECURITY EVALUATION USING CAPACITY TABLE APPROACH}

\subsection{INTRODUCTION}

In a practical power system, the load changes continuously and therefore it is not economical to run all the generating units required to satisfy the peak load during the low load periods. Usually, some units are put into service at one time period and removed from service or additional units are added to the service at another time period depending on the load level. The generating units should be committed to service for different segments of the scheduling period in such a way that the operating cost is minimized at a satisfactory level of reliability. The unit commitment should be such that the commitment risk should be less than or equal to a specified level. In a composite generation and transmission system, the unit commitment should satisfy both the HLI risk level [83] and the composite system risk level CSOSR as defined in Chapter 7. The risk value in a composite system will be different from the risk value at HLI due to the transmission line constraints, location of generating units, etc.. Specified acceptable risk levels for both HLI and composite system evaluations could be utilized and the unit commitment such that both risk values are satisfied. It might be possible that the units committed in service satisfy the HLI risk criterion and not satisfy the composite system risk requirement. In this case, more generating units should be brought into service to satisfy both risk criteria.

The fundamental difference between static and operating capacity evaluation is in the time period considered. Static capacity evaluation is done for long term system requirements whereas operating capacity evaluation is a short term capacity assessment to meet a given load demand. The time period used in an operating capacity evaluation is generally relatively small and known as the lead time. This is the time period for which 
no additional units can be brought into service. The lead time can be a few minutes to several hours depending on the type and size of the unit to be brought into service. The effect of unit commitment and the lead time on the indices of different operating states have been considered in this chapter. The main objective of this chapter is to demonstrate the effect of the number of units committed in an HLI study and the lead time on the probabilities of different operating states and on the composite system risk. The lead time for all generating units is considered to be equal to simplify the calculation and to reduce the computation time. The effect of rapid start units, derated states, postponable outages and load forecast uncertainty can all be included if desired [2]. The object in this analysis is to illustrate the utilization of the CSOSR in the unit commitment problem. The total generating unit state probability considered in the studies can be increased by using a capacity outage probability table instead of using the individual generating units. The utilization of a capacity outage probability table to calculate the indices of different operating states is demonstrated in the following section.

\subsection{ANALYSIS OF DIFFERENT STATES USING A CAPACITY OUTAGE PROBABILITY TABLE}

The operating state probabilistic indices were calculated in the previous chapters by considering each generating units separately. These indices can also be calculated using a capacity outage probability table for the generating units instead of using each individual unit. Before presenting the algorithm for this purpose, the procedure for calculating a capacity outage probability table is briefly discussed in the next subsection.

\subsubsection{Capacity Outage Probability Table}

The system generating units can be represented by a equivalent generating source which may have different capacity output states depending on the capacity outage states and the corresponding probabilities of the individual generating units. A capacity outage probability table [2] is a systematic representation of this equivalent generation source. The capacity outage can be created using the simple recursive algorithm given by Equation 8.1. 


$$
\begin{aligned}
& p(X)=\sum_{i=1}^{n} p^{\prime}\left(X-C_{i}\right) p_{i} \\
& \lambda_{+}(X)=\frac{\sum_{i=1}^{n} p^{\prime}\left(X-C_{i}\right) p_{i}\left(\lambda_{+}^{\prime}\left(X-C_{i}\right)+\lambda_{+}\left(C_{i}\right)\right)}{p(X)} \\
& \lambda_{-}(X)=\frac{\sum_{i=1}^{n} p^{\prime}\left(X-C_{i}\right) p_{i}\left(\lambda_{-}^{\prime}\left(X-C_{i}\right)+\lambda_{-}\left(C_{i}\right)\right)}{p(X)}
\end{aligned}
$$

where:

$\mathrm{C}_{\mathrm{i}} \quad$ = capacity outage of state $\mathrm{i}$ for the unit being added

$\mathrm{p}_{\mathrm{i}} \quad=$ probability of of the $\mathrm{i}^{\text {th }}$ state for the unit

$\mathrm{n} \quad=$ number of capacity outage state of the unit

$\begin{aligned} \mathrm{p}(\mathrm{X})= & \text { individual state probability of the capacity outage state of exactly } \\ & \mathrm{X} \mathrm{MW} \text { out after the unit is added }\end{aligned}$

$\lambda_{+}(X)=$ the upward departure rate of exactly $X \mathrm{MW}$ out after the unit is added

$\lambda_{-}(\mathrm{X})=$ the downward departure rate of exactly $\mathrm{X}$ MW out after the unit is added

The primed values in Equation 8.1 represent the similar quantities as noted above before the unit is added. In Equation 8.1, if $\mathrm{X}$ is less than $\mathrm{C}$,

$$
\begin{array}{ll}
\mathrm{p}^{\prime}(\mathrm{X}-\mathrm{C}) & =0 \\
\lambda_{+}^{\prime}(\mathrm{X}-\mathrm{C}) & =0 \\
\lambda_{-}^{\prime}(\mathrm{X}-\mathrm{C}) & =0
\end{array}
$$

The capacity outage probability table incorporate all possible generating sates. The identical states can be combined as follows. For a given margin state $m_{k}$ made up to $s$ identical states 


$$
\begin{aligned}
& p_{k}=\sum_{i=1}^{s} p_{i} \\
& \lambda_{ \pm k}=\frac{\sum_{i=1}^{s} p_{i} \lambda_{ \pm i}}{p_{k}}
\end{aligned}
$$

The table can be truncated by calculating the cumulative probabilities in each step and neglecting the states whose cumulative probabilities are less than a specified values. The cumulative probability can be calculated using the following relations

$$
\begin{aligned}
& P(1)=1.0 \\
& P(i+1)=P(i)-p(i) \text { for } i=1,2,3 \ldots . . N
\end{aligned}
$$

where:

$$
\begin{aligned}
& \mathrm{N}=\text { the number of states in the capacity outage probability table } \\
& \mathrm{P}=\text { the cumulative probability } \\
& \mathrm{p}=\text { the individual probability }
\end{aligned}
$$

\subsubsection{Use Of A Capacity Table For Calculating The Indices}

All the generating units at HLI in both adequacy and security analysis are considered to be connected to a single bus in order to develop the system capacity outage probability table. Transmission lines are not normally considered at HLI. In a composite system study the transmission configuration is an important factor and therefore, the generating units and the different bus loads can not be considered to be connected to a single bus. A capacity outage probability table must be developed for each of the buses where generating units are actually connected. The different states of the capacity outage probability tables can be treated as individual units and studies conducted using the methods described earlier. Multiple level outages are considered by combining the states from different buses. The two test systems have been analyzed using the above technique and the results are presented below. 


\section{Results for the MRBTS}

As noted earlier, this system has two generating buses and 11 generating units. Four generators are connected at bus 1 and the remaining seven generators are connected at bus 2 . Two capacity outage probability tables, one at bus 1 and the other at bus 2 , are required for this system. These capacity outage probability tables at buses 1 and 2 are shown in Tables 8.1 and 8.2 respectively. Considering each state from Tables 8.1 and 8.2

Table 8.1: Capacity outage probability table at bus 1 - MRBTS

\begin{tabular}{rrrr}
\hline State & $\begin{array}{c}\text { Capacity } \\
\text { Out }(\mathrm{MW})\end{array}$ & $\begin{array}{l}\text { Individual } \\
\text { Probability }\end{array}$ & $\begin{array}{c}\text { Total Departure } \\
\text { Rate (f/yr) }\end{array}$ \\
\hline 1 & 0.0 & 0.8990531 & 21.0000 \\
2 & 10.0 & 0.0184737 & 211.6667 \\
3 & 20.0 & 0.0230921 & 210.6667 \\
4 & 30.0 & 0.0004745 & 401.3333 \\
5 & 40.0 & 0.0554211 & 209.6667 \\
6 & 50.0 & 0.0011388 & 400.3333 \\
7 & 60.0 & 0.0014235 & 399.3333 \\
8 & 70.0 & 0.0000292 & 590.0000 \\
9 & 80.0 & 0.0008541 & 398.3333 \\
10 & 90.0 & 0.0000175 & 589.0000 \\
11 & 100.0 & 0.0000219 & 588.0000 \\
12 & 110.0 & 0.0000005 & 778.6667 \\
\hline
\end{tabular}

as a generating unit and using the same flow chart given in Chapter 4 for state detection, the indices for different operating states are calculated and shown in Table 8.3. These indices can be compared with those obtained by considering each of the generating units separately (as in Chapter 5) for the total constraint set. It can be seen from these results and those given in Chapter 5 that the probabilities and frequencies of the normal and alert states are quite different. The different states of the capacity outage probability tables are treated as generating units and therefore the combination of low capacity outage states with the higher capacity outage states create system problems and therefore these low 
Table 8.2: Capacity outage probability table at bus 2 - MRBTS

\begin{tabular}{rrrr}
\hline State & $\begin{array}{c}\text { Capacity } \\
\text { Out (MW) }\end{array}$ & $\begin{array}{l}\text { Individual } \\
\text { Probability }\end{array}$ & $\begin{array}{c}\text { Total Departure } \\
\text { Rate (f/yr) }\end{array}$ \\
\hline 1 & 0.0 & 0.9042882 & 16.6000 \\
2 & 5.0 & 0.0185813 & 209.2667 \\
3 & 10.0 & 0.0000955 & 401.9333 \\
4 & 20.0 & 0.0545050 & 173.4727 \\
5 & 25.0 & 0.0011200 & 366.1394 \\
6 & 30.0 & 0.0000058 & 558.8061 \\
7 & 40.0 & 0.0198132 & 170.2168 \\
8 & 45.0 & 0.0004071 & 362.8834 \\
9 & 50.0 & 0.0000021 & 555.5501 \\
10 & 60.0 & 0.0011323 & 318.3389 \\
11 & 65.0 & 0.0000233 & 511.0055 \\
12 & 70.0 & 0.0000001 & 703.6722 \\
13 & 80.0 & 0.0000254 & 473.6593 \\
14 & 85.0 & 0.0000005 & 666.3260 \\
15 & 90.0 & 0.0000000 & 858.9927 \\
16 & 100.0 & 0.0000003 & 630.2182 \\
17 & 105.0 & 0.0000000 & 822.8848 \\
18 & 110.0 & 0.0000000 & 1015.5515 \\
19 & 120.0 & 0.0000000 & 787.0909 \\
20 & 125.0 & 0.0000000 & 979.7576 \\
21 & 130.0 & 0.0000000 & 1172.4242 \\
\hline
\end{tabular}

capacity outage states are assigned to the alert state. This was not the case when the generating units were considered as individual components in Chapter 5. The use of capacity outage probability tables provides a more accurate CSOSR due to the inclusion of the higher level outages. Similarly, the indices for the extreme emergency state will also be more comprehensive. The indices for the extreme emergency state are higher than those given in Chapter 5 due to the fact that more higher level generator and generator plus line outages are considered in the studies using capacity outage probability tables. The total of the operating state probabilities is also higher in Table 8.3 than that experience earlier. 
Table 8.3: Probability and frequency of different operating states - MRBTS

\begin{tabular}{lrr}
\hline $\begin{array}{l}\text { System } \\
\text { State }\end{array}$ & Probability & Frequency \\
& & \\
\hline Normal & 0.796787 & 47.966580 \\
Alert & 0.194366 & 61.092001 \\
Emergency & 0.000137 & 0.207563 \\
Ext. Emergency & 0.008607 & 4.010731 \\
No problem & 0.000100 & 0.140290 \\
\hline
\end{tabular}

Total considered $\quad 0.999997$

\section{Result for the IEEE-RTS}

This test system has 32 generating units connected to $10 \mathrm{PV}$ buses. Capacity outage probability tables similar to those used in the MRBTS have been developed for these 10 PV buses. The indices obtained for the different operating states are shown in Table 8.4. The total of the operating state probabilities obtained using this method is higher than that encountered earlier as more higher level outages are included in the analysis. The probability of the extreme emergency state is also higher due to factors noted earlier.

\subsection{LEAD TIME AND ITS EFFECT ON THE OPERATING STATES}

The results presented in the previous section indicate that the indices calculated by the capacity outage probability table approach are more comprehensive than those obtained earlier using each generating unit as an individual component. The capacity table concept can be utilized to calculate spinning or operating capacity risk indices related to the different operating states. In the previous analyses, the generating risk limiting state probabilities were used to derive the capacity outage probability tables. In a operating capacity risk study, however, the generating unit time dependent probabilities 
Table 8.4: Probability and frequency of different operating states - IEEE-RTS

\begin{tabular}{lrr}
\hline $\begin{array}{l}\text { System } \\
\text { State }\end{array}$ & Probability & Frequency \\
& & \\
\hline Normal & 0.000000 & 0.000000 \\
Alert & 0.886093 & 403.222864 \\
Emergency & 0.001307 & 0.896533 \\
Ext. Emergency & 0.090812 & 58.447263 \\
No problem & 0.013289 & 13.509716 \\
\hline & & \\
\hline Total considered & 0.991501 & \\
\hline
\end{tabular}

are used to derive the system capacity outage probability table. The time dependent probabilities can be calculated using an appropriate operating unit model [2]. The studies are reported in the next section.

\subsection{SPINNING RESERVE ASSESSMENT IN HLI}

Probabilistic techniques are available to evaluate the unit commitment and spinning reserve requirements in a power system at HLI [2]. The main objective in such a study is to keep the unit commitment risk equal to or less than a specified value throughout a given period. The probabilistic technique permits recognition of the random behaviour of system components and incorporates it in a consistent evaluation of the spinning reserve requirements. The unit commitment risk can be decreased by committing i.e. operating more generation capacity for the same load demand. The selection of an acceptable risk level depends on the desired degree of reliability, the corresponding cost and the optimum benefits. The unit commitment risk can be expressed as

$$
U(t)=\sum_{i=1}^{N} P_{i}(t) Q_{i}(t)
$$

where:

$\mathrm{U}(\mathrm{t})=$ system risk at time $\mathrm{t}$ 
$P_{i}(t)=$ probability that the system is in state $i$ at time $t$

$\mathrm{Q}_{\mathbf{i}}(\mathrm{t})=$ probability that the system load will be equal to or greater than the generation at state $i$ at time $t$

$\mathrm{N}=$ total number of system states

In the case of an operating or spinning reserve study $Q_{i}$ becomes either zero or unity.

$$
\begin{array}{ll}
Q_{i}(t)=0 & \text { when } L<C_{i} \\
Q_{i}(t)=1 & \text { when } L \geq C_{i}
\end{array}
$$

where:

$\mathrm{L} \quad=$ system load and

$\mathrm{C}_{\mathrm{i}}=$ total spinning capacity of the system in the $\mathrm{i}^{\text {th }}$ state

The capacity outage probability table can be arranged such that

$$
C_{i}>C_{i+1} \quad i=1,2,3 \ldots \ldots, N-1
$$

where $\mathrm{N}$ is the total number of states in the generation system. Equation 8.2 can be simplified as

$$
U(t)=\sum_{i=n}^{N} P_{i}(t)
$$

where $n$ is an integer such that $\left(L-C_{n}\right) \geq 0$ and $\left(L-C_{n-1}\right)<0$, i.e. $C_{n-1}>L \geq C_{n}$. Therefore, $U(t)$ is the cumulative probability of the generation states $n$ at time $t$. The unit commitment in a system for an allowable risk $R_{s}$ for a time period $(0, t)$ should follow

$$
\mathrm{U}(\mathrm{t}) \leq \mathrm{R}_{\mathrm{s}}
$$

The unit commitment and the associated unit commitment risk are based on the fact that additional generation will become available after a certain period of time designated as 
the system lead time $[2,108]$. The risk is considered to be the probability of just carrying or failing to carry the load in a HLI study [2]. The unit commitment basically depends on system load, generating unit failure rates, system lead time and the acceptable unit commitment risk level.

\subsubsection{Unit Commitment In HLI}

A probabilistic approach to unit commitment in a single system requires that the unit commitment risk should be less than or equal to a specified risk level for all forecast load levels. In practice, an operator would use the probabilistic risk assessment method by adding i.e. committing one unit at a time from the merit order table until the unit commitment risk given by the generation model becomes equal to or less than the acceptable level for the expected load. One of the most important parameters in the assessment of unit commitment risk is the time delay or lead time after which the additional generation will be available.

\subsubsection{Results For The MRBTS}

The number of generators to be committed for a particular load level are usually taken from a priority unit loading order table. This table is prepared based on economy and system operating factors. The priority list approach to unit commitment is used by many utilities. The principle advantages of this technique is that it is simple, straight forward and can be applied to a system with a large number of units. As the load increases, individual units are committed according to a pre-determined priority order. Units are removed from service as the load decreases using the reverse priority order. The priority order list of the generating units for the RBTS [91] is shown in Table 8.5. Using this priority order list, the number of generators required for a $60 \%$ peak load level in the MRBTS for an HLI study is shown in Table 8.6 for different lead times. The unit commitment is made using a risk level of 0.001 . The indices for different system operating states have been calculated using the same number of generating units and the corresponding lead times in Table 8.6, and are presented in Figures 8.1 and 8.2. It can be seen that as the lead time increases, the probability of the normal state decreases whereas the probability of the alert state increases. The probability of the extreme emergency 
Table 8.5: Priority loading order list of generating units - MRBTS

\begin{tabular}{rrrrr}
\hline $\begin{array}{c}\text { Priority } \\
\text { Order }\end{array}$ & $\begin{array}{c}\text { Generator } \\
\text { Number }\end{array}$ & $\begin{array}{c}\text { Capacity } \\
\text { (MW) }\end{array}$ & Type & $\begin{array}{c}\text { Connected } \\
\text { At Bus }\end{array}$ \\
\hline 1 & 7 & 40 & hydro & 2 \\
2 & 8 & 20 & hydro & 2 \\
3 & 9 & 20 & hydro & 2 \\
4 & 1 & 40 & thermal & 1 \\
5 & 2 & 40 & thermal & 1 \\
6 & 4 & 20 & thermal & 1 \\
7 & 3 & 10 & thermal & 1 \\
8 & 10 & 20 & hydro & 2 \\
9 & 11 & 20 & hydro & 2 \\
10 & 5 & 5 & hydro & 2 \\
11 & 6 & 5 & hydro & 2 \\
\hline
\end{tabular}

Table 8.6: Number of generators required for different lead time with fixed load level - MRBTS

\begin{tabular}{ccccc}
\hline $\begin{array}{c}\text { Load } \\
(\mathrm{MW})\end{array}$ & $\begin{array}{c}\text { Desired } \\
\text { Risk }\end{array}$ & $\begin{array}{c}\text { Lead Time } \\
\text { (Minutes) }\end{array}$ & $\begin{array}{c}\text { No. Of Unit } \\
\text { Required }\end{array}$ & $\begin{array}{c}\text { Actual } \\
\text { Risk }\end{array}$ \\
\hline 111 & 0.001 & 30 & 4 & $0.00078745-P_{\text {. }}=0$ \\
111 & 0.001 & 60 & 5 & 0.00000188 \\
111 & 0.001 & 120 & 5 & 0.00000749 \\
111 & 0.001 & 180 & 5 & 0.00001685 \\
111 & 0.001 & 240 & 5 & 0.00002993 \\
111 & 0.001 & 300 & 5 & 0.00004673 \\
111 & 0.001 & 900 & 5 & 0.00041725 \\
\hline
\end{tabular}

state increases as the lead time increases. The probability of the extreme emergency state decreases as the lead time increases from 0.5 hour to 1 hour as the number of units committed to the system is different for these two lead times as shown in Table 8.6. Four 

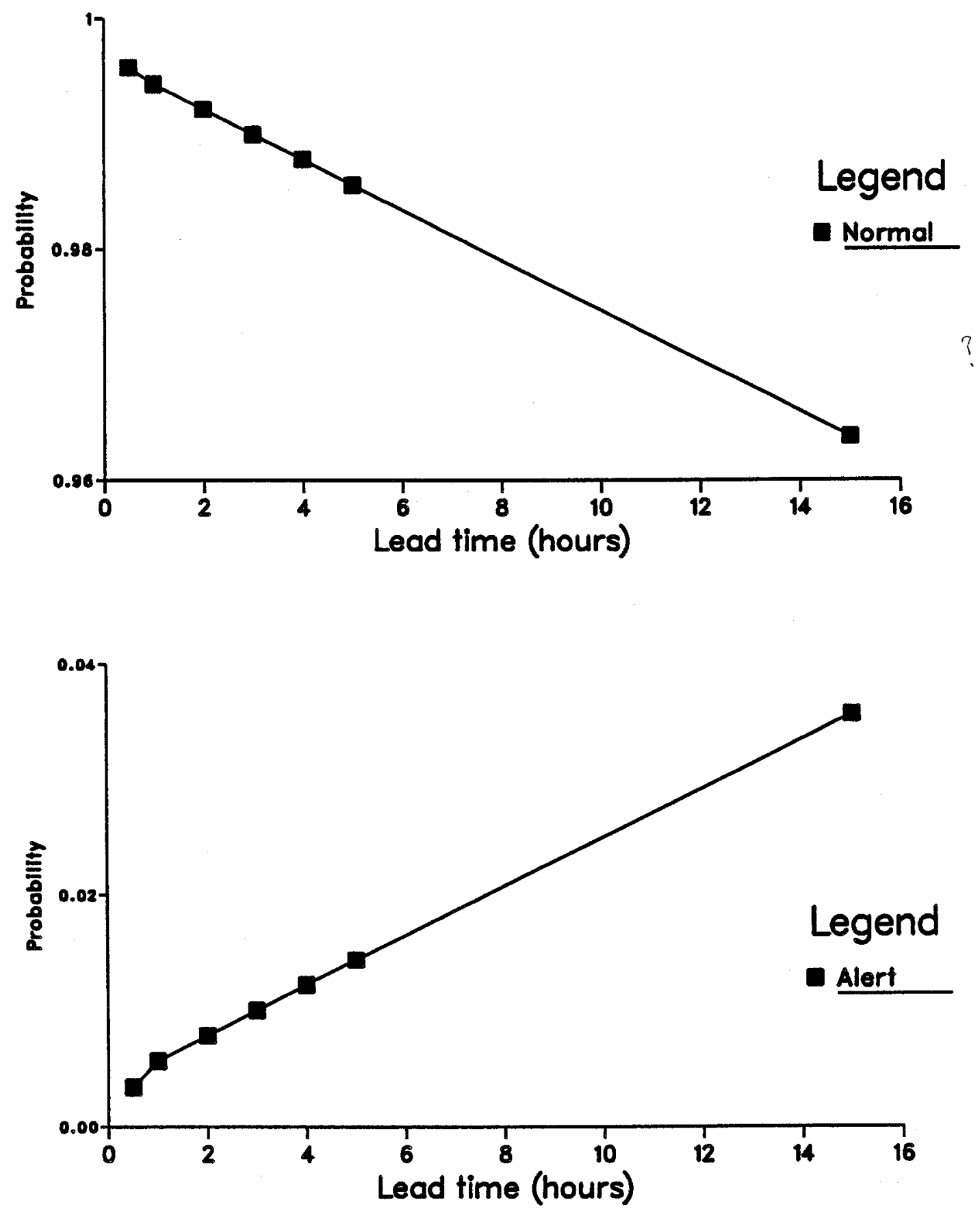

Figure 8.1: Probability of the normal and alert states - MRBTS 

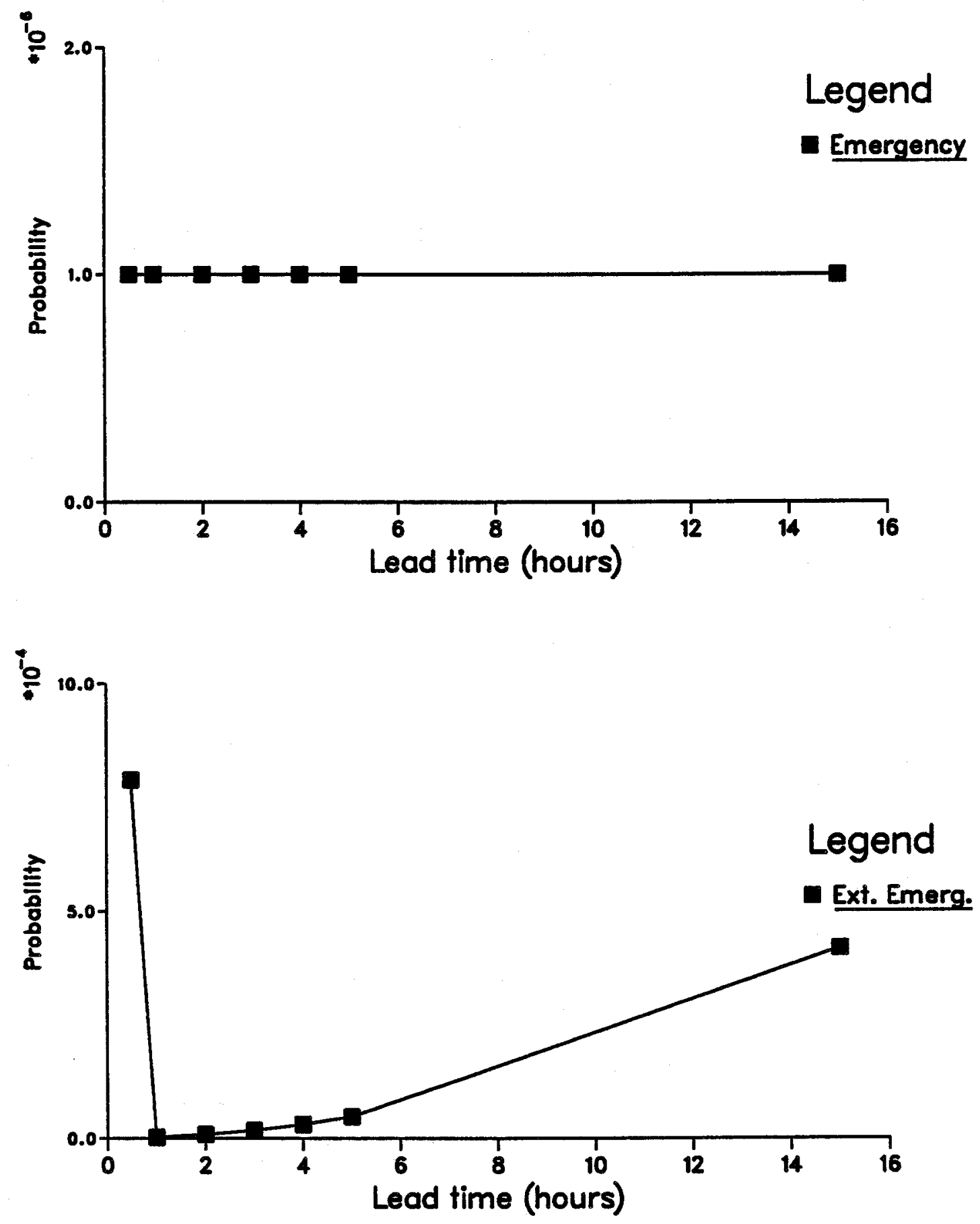

Figure 8.2: Probability of the emergency and extreme emergency states - MRBTS 
units are committed to the system for a lead time of 0.5 hour, whereas five units are committed for a 1-hour lead time. These results show that for a fixed number of committed units, the system operating state indices are very sensitive to the lead time for this test system as most of the problem creating contingencies result in load curtailment.

Another study was conducted in which the lead time was fixed at 4 hours and the system peak load varied. The number of units required for the specified unit commitment risk for different peak loads are shown in Table 8.7. Indices for different system

Table 8.7: Number of generators required for different peak load with fixed lead time - MRBTS

\begin{tabular}{ccccc}
\hline $\begin{array}{c}\text { Load } \\
(\mathrm{MW})\end{array}$ & $\begin{array}{c}\text { Desired } \\
\text { Risk }\end{array}$ & $\begin{array}{c}\text { Lead Time } \\
\text { (Minutes) }\end{array}$ & $\begin{array}{c}\text { No. Of Unit } \\
\text { Required }\end{array}$ & $\begin{array}{c}\text { Actual } \\
\text { Risk }\end{array}$ \\
\hline 74.0 & 0.001 & 240 & 4 & 0.00001274 \\
92.5 & 0.001 & 240 & 5 & 0.00001500 \\
111.0 & 0.001 & 240 & 5 & 0.00002993 \\
129.5 & 0.001 & 240 & 6 & 0.00004547 \\
148.0 & 0.001 & 240 & 7 & 0.00005788 \\
166.5 & 0.001 & 240 & 8 & 0.00006532 \\
185.0 & 0.001 & 240 & 9 & 0.00007276 \\
\hline
\end{tabular}

operating states were calculated using this table and are shown in Table 8.8. It can be seen that the number of units committed in the basic HLI study provides an acceptable CSOSR with a slightly higher value than in the HLI study. The specified CSOSR could be the same as the HLI unit commitment risk of 0.001 or if desired some other value. In this case, if the desired risk level is 0.001 , the units committed on the basis of this risk level result in an actual risk which is much lower due to the units committed. The unit commitment risk for a peak load of $166.5 \mathrm{MW}$ and a lead time of 15 hours is 0.00090485 for the MRBTS and requires 8 generating units to be committed to the system. In this case, this risk value from the HLI study is very close to the acceptable risk level of 0.001 . The corresponding CSOSR from a composite system study is 0.001086 which is higher than 0.001 . Therefore, if the same risk level is defined as being acceptable for both HLI 
Table 8.8: Effect of variation of load on the probability of different states with fixed lead time - MRBTS

\begin{tabular}{|c|c|c|c|c|c|c|}
\hline \multirow{2}{*}{$\begin{array}{l}\text { Load } \\
\text { (MW) }\end{array}$} & \multicolumn{2}{|c|}{ 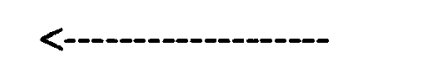 } & \multirow{2}{*}{$\begin{array}{l}\text { Probability of } \\
\text { Emergency }\end{array}$} & & \multirow[b]{2}{*}{ CSOSR } \\
\hline & Normal & Alert & & $\begin{array}{c}\text { Ext. } \\
\text { Emergency }\end{array}$ & $\begin{array}{c}\text { No } \\
\text { Problem }\end{array}$ & \\
\hline 74.0 & 0.991565 & 0.008419 & 0.000000 & 0.000014 & 0.000002 & 0.000014 \\
\hline 92.5 & 0.989900 & 0.010078 & 0.000003 & 0.000016 & 0.000003 & 0.000019 \\
\hline 111.5 & 0.987748 & 0.012216 & 0.000001 & 0.000031 & 0.000003 & 0.000033 \\
\hline 129.5 & 0.985524 & 0.014424 & 0.000001 & 0.000047 & 0.000003 & 0.000048 \\
\hline 148.0 & 0.969314 & 0.030569 & 0.000004 & 0.000109 & 0.000003 & 0.000114 \\
\hline 166.5 & 0.964886 & 0.034906 & 0.000082 & 0.000124 & 0.000002 & 0.000207 \\
\hline 185.0 & 0.963810 & 0.035974 & 0.000082 & 0.000135 & 0.000002 & 0.000218 \\
\hline
\end{tabular}

Total probability considered in all cases $=1.000000$.

and composite system studies, then one more generating unit should be committed to the system for this particular load level and lead time as shown in Table 8.9. This table shows the variation of risk with the number of units for a load level of $166.5 \mathrm{MW}$. The composite system risk is 0.000327 with nine generating units committed to the system. It

Table 8.9: CSOSR at the $166.5 \mathrm{MW}$ load level - MRBTS

Number Of

Unit Committed
CSOSR

0.001086

0.000327

. may be desirable to specify a different risk level for composite system analysis, depending upon the system, as the calculated CSOSR will be always higher than the HLI unit commitment risk. This will require further study. 


\subsubsection{Results For The IEEE-RTS}

The number of generating units to be committed for a particular load level were taken from the priority order list [83] for this test system as shown in Table 8.10. The

Table 8.10: Priority loading order list of generating units - IEEE-RTS

\begin{tabular}{rrrrr}
$\begin{array}{r}\text { Priority } \\
\text { Order }\end{array}$ & $\begin{array}{c}\text { Generator } \\
\text { Number }\end{array}$ & $\begin{array}{c}\text { Capacity } \\
\text { (MW) }\end{array}$ & Type & $\begin{array}{c}\text { Connected } \\
\text { At Bus }\end{array}$ \\
$1-4$ & $1-4$ & 50 & hydro & 22 \\
5 & 30 & 400 & nuclear & 18 \\
6 & 31 & 400 & nuclear & 21 \\
7 & 29 & 350 & thermal & 23 \\
$8-10$ & $16-18$ & 187 & thermal & 13 \\
11 & 12 & 155 & thermal & 15 \\
$12-13$ & $27-28$ & 155 & thermal & 23 \\
14 & 32 & 155 & thermal & 16 \\
$15-17$ & $13-15$ & 100 & thermal & 7 \\
$18-19$ & $21-22$ & 76 & thermal & 1 \\
$20-21$ & $25-26$ & 76 & thermal & 2 \\
$22-26$ & $7-11$ & 12 & thermal & 15 \\
$27-28$ & $19-20$ & 20 & thermal & 1 \\
$29-30$ & $23-24$ & 20 & thermal & 2 \\
$31-32$ & $5-6$ & 50 & hydro & 22 \\
& & & & \\
\hline
\end{tabular}

number of generating units required for a load level of $70 \%$ of the peak load of $2850 \mathrm{MW}$ with different lead times and on unit commitment risk of 0.001 are shown in Table 8.11. The probabilities of different system operating states were calculated considering the same number of generating units and lead times and the results are presented in Figures 8.3 and 8.4. The probability of the normal state is zero for all the lead times since single level events create system voltage problems. The probability of the alert state decreases as the lead time increases. The probabilities of the emergency and extreme emergency states increase with the lead time. The variation is not linear although the number of generating units for lead times up to 5 hours are identical. The change in the indices for the emergency and extreme emergency states at a lead time of 15 hours is due to the commitment of another unit. 
Table 8.11: Number of generators required for different lead time with fixed load level - IEEE-RTS

\begin{tabular}{ccccc}
\hline $\begin{array}{l}\text { Load } \\
(\mathrm{MW})\end{array}$ & $\begin{array}{c}\text { Desired } \\
\text { Risk }\end{array}$ & $\begin{array}{c}\text { Lead Time } \\
\text { (Minutes) }\end{array}$ & $\begin{array}{c}\text { No. Of Unit } \\
\text { Required }\end{array}$ & $\begin{array}{c}\text { Actual } \\
\text { Risk }\end{array}$ \\
\hline 1995 & 0.001 & 30 & 13 & 0.00000573 \\
1995 & 0.001 & 60 & 13 & 0.00002288 \\
1995 & 0.001 & 120 & 13 & 0.00009123 \\
1995 & 0.001 & 180 & 13 & 0.00020458 \\
1995 & 0.001 & 240 & 13 & 0.00036247 \\
1995 & 0.001 & 300 & 13 & 0.00056446 \\
1995 & 0.001 & 600 & 14 & 0.00084794 \\
1995 & 0.001 & 900 & 15 & 0.00072963 \\
\hline
\end{tabular}

A similar study to that conducted in the MRBTS was performed for the IEEE-RTS using seven load levels and a fixed lead time of 4 hours. The number of generating units committed and the corresponding HLI unit commitment risk are shown in Table 8.12. The indices for the different system operating states for these seven levels using the corresponding units from Table 8.12 are shown in Table 8.13. The corresponding risk values from the HLI and composite system studies are shown in Figure 8.5. It can be seen from this figure that the CSOSR is always higher than the risk value from the HLI studies as expected. The CSOSR is below the acceptable limit of 0.001 up to the $90 \%$ load level for the same number of generating units committed in the HLI studies. At the $100 \%$ load level, the risk is higher than 0.001 with 28 generating units committed to the system. The CSOSR with different numbers of generating units at this load level is shown in Table 8.14. It can be seen from this table that 3 additional generating units should be committed for an acceptable CSOSR of 0.001 . 

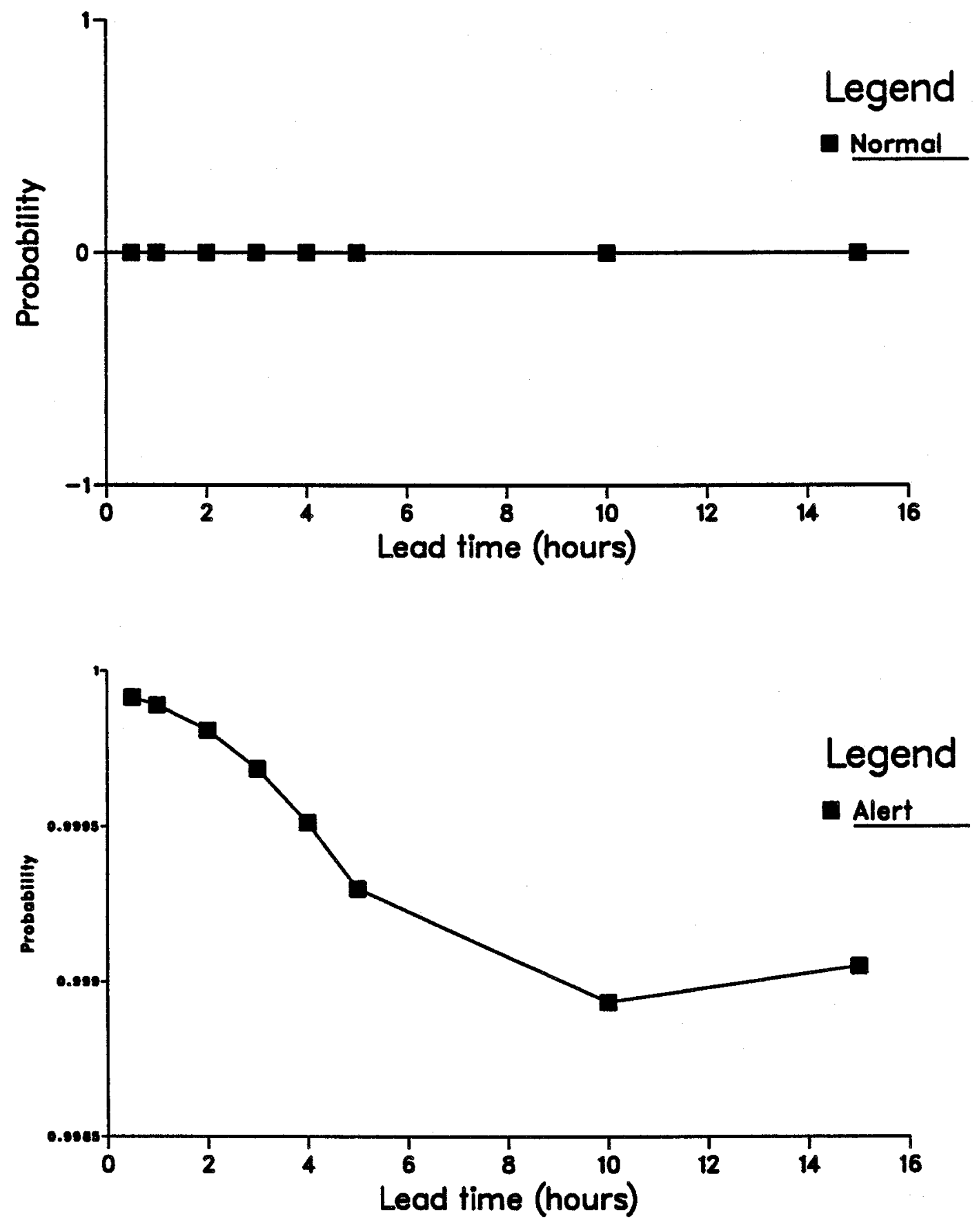

Figure 8.3: Probability of the normal and alert states - IEEE-RTS 

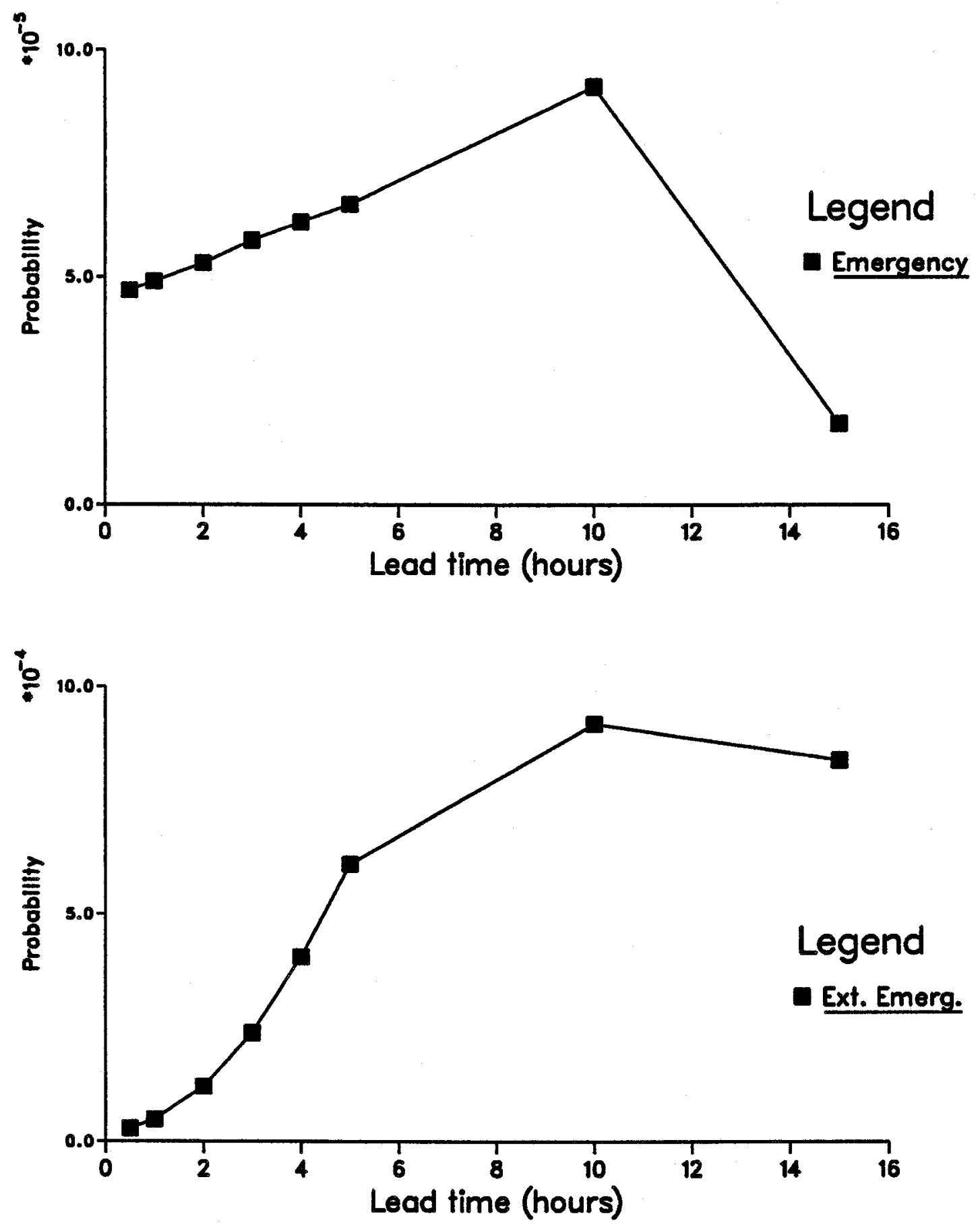

Figure 8.4: Probability of the emergency and extreme emergency states - IEEERTS 
Table 8.12: Number of generators required for different peak load with fixed lead time - IEEE-RTS

\begin{tabular}{ccccc}
\hline $\begin{array}{c}\text { Load } \\
(\mathrm{MW})\end{array}$ & $\begin{array}{c}\text { Desired } \\
\text { Risk }\end{array}$ & $\begin{array}{c}\text { Lead Time } \\
\text { (Minutes) }\end{array}$ & $\begin{array}{c}\text { No. Of Unit } \\
\text { Required }\end{array}$ & $\begin{array}{c}\text { Actual } \\
\text { Risk }\end{array}$ \\
\hline 1140 & 0.001 & 240 & 8 & 0.00014127 \\
1425 & 0.001 & 240 & 10 & 0.00017271 \\
1710 & 0.001 & 240 & 12 & 0.00023273 \\
1995 & 0.001 & 240 & 13 & 0.00036247 \\
2280 & 0.001 & 240 & 16 & 0.00039689 \\
2565 & 0.001 & 240 & 19 & 0.00053997 \\
2850 & 0.001 & 240 & 28 & 0.00071863 \\
\hline
\end{tabular}

Table 8.13: Effect of load variation on the probability of different states with fixed lead time - IEEE-RTS

\begin{tabular}{|c|c|c|c|c|c|c|}
\hline \multirow{2}{*}{$\begin{array}{l}\text { Load } \\
\text { (MW) }\end{array}$} & \multirow{2}{*}{ Normal } & \multicolumn{2}{|c|}{ Probability of } & \multicolumn{2}{|c|}{ 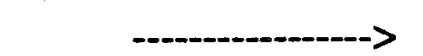 } & \multirow[b]{2}{*}{ CSOSR } \\
\hline & & Alert & Emergency & $\begin{array}{c}\text { Ext. } \\
\text { Emergency }\end{array}$ & $\begin{array}{c}\text { No } \\
\text { Problem }\end{array}$ & \\
\hline 1140 & 0.961206 & 0.038607 & 0.000016 & 0.000146 & 0.000010 & 0.000177 \\
\hline 1425 & 0.961420 & 0.038358 & 0.000011 & 0.000178 & 0.000015 & 0.000207 \\
\hline 1710 & 0.941786 & 0.057920 & 0.000011 & 0.000240 & 0.000022 & 0.000272 \\
\hline 1995 & 0.000000 & 0.999489 & 0.000063 & 0.000404 & 0.000022 & 0.000489 \\
\hline 2280 & 0.000000 & 0.999360 & 0.000137 & 0.000444 & 0.000032 & 0.000608 \\
\hline 2565 & 0.000000 & 0.999196 & 0.000146 & 0.000588 & 0.000039 & 0.000765 \\
\hline 2850 & 0.000000 & 0.998732 & 0.000234 & 0.000916 & 0.000072 & 0.001195 \\
\hline
\end{tabular}




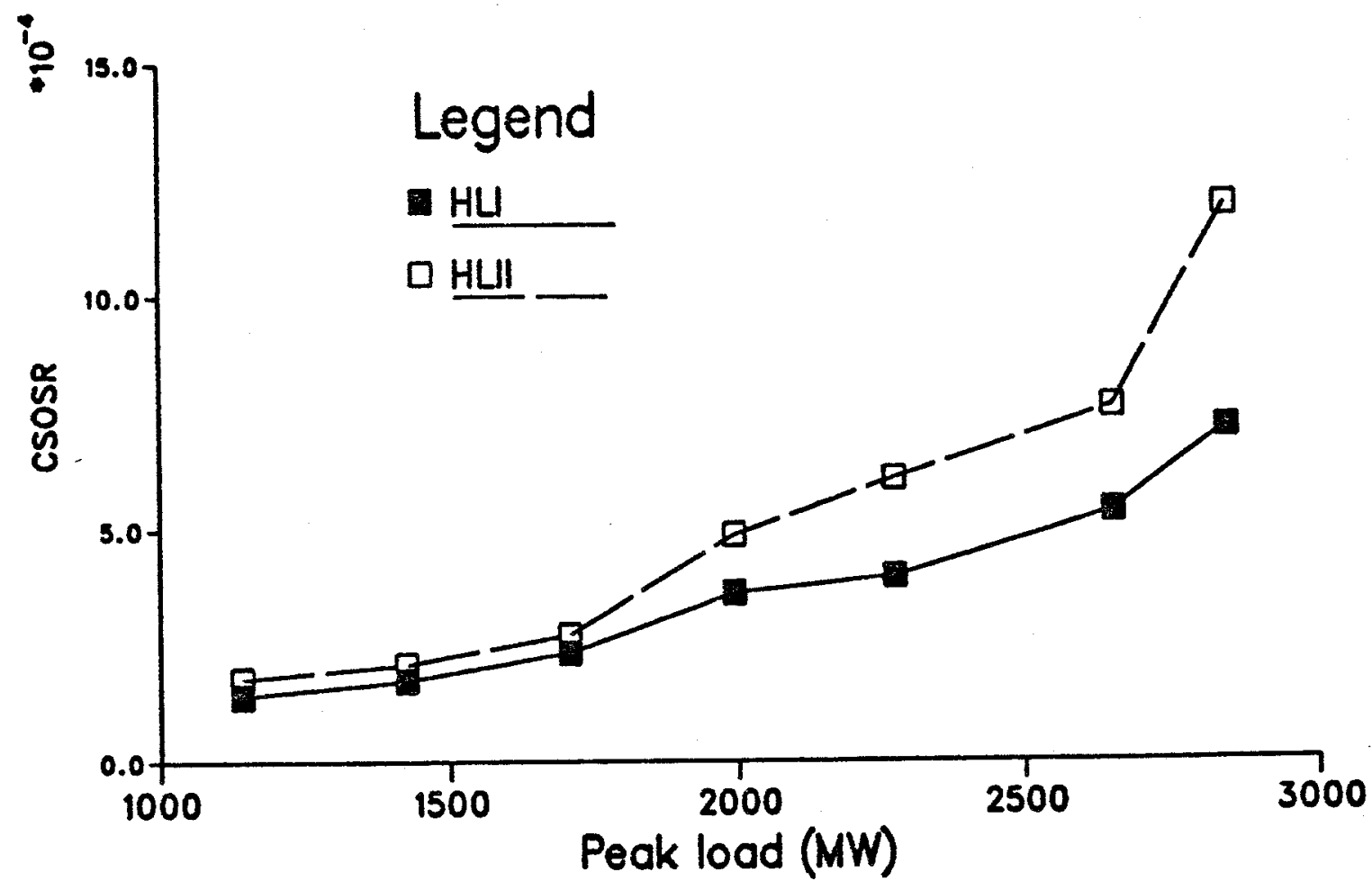

Figure 8.5: Variation of risks with the lead time - IEEE-RTS

Table 8.14: CSOSR at the $2850 \mathrm{MW}$ load level - IEEE-RTS

Number Of

Unit Committed
CSOSR

0.001195

0.001085

0.001028

0.000910 


\subsection{SUMMARY}

A unit commitment method considering the composite generation and transmission system is discussed in this chapter. The generating units are committed first by considering the acceptable HLI unit commitment risk. The CSOSR is evaluated with the committed generating units and tested against the acceptable value. The number of units committed should satisfy both acceptable risk levels. The method uses a priority loading order for the generating units to be committed during the period of increasing generation requirements.

There is no straightforward relationship between the required number of generating units, specified unit commitment risk and load level. The required number of units is a complex function of unit size, unit failure rate, lead time and specified risk level. The number of units required for a given load level can be reduced by decreasing the lead time of additional generation provided other variables remain the same. The effects of lead time on the number of units for a given load level are presented in this chapter. The number of units committed for a particular load level in an HLI study may or may not satisfy the CSOSR. It has been shown that the composite system risk is always higher than the HLI risk due to transmission line constraints, location of generating units, etc.. The acceptable risk indices for HLI and the composite system should, therefore, perhaps be different. In the studies reported in this chapter, however, equal risk levels are considered in order to simply demonstrate the required number of units that should be committed from both HLI and composite system points of view. The results for different lead times for a fixed load level and for variable load levels for a fixed lead time are also presented in this chapter. The lead time is considered to be equal for all generating units in the studies reported in this chapter. This lead time could be different for different units depending on the types and sizes of the units. 


\section{CONCLUSIONS}

A new technique for reliability assessment of composite generation and transmission systems is proposed and illustrated in this thesis. The conventional indices in the adequacy domain are calculated using three solution techniques. A technique is developed to reduce the computation time by limiting the number of possible contingencies for further investigation using the ac load flow method without introducing significant errors in the final indices. Quantitative indices for different system operating states are calculated and extended using analytical and hybrid techniques. A Composite System Operating State Risk (CSOSR) is defined and several examples of possible utilization of the CSOSR in system expansion planning and unit commitment are demonstrated.

The most basic technique for composite system reliability evaluation is the enumeration method which involves systematic selection and evaluation of contingencies, the classification of each contingency in accordance with selected failure criteria and the accumulation of the indices. There are several solution techniques that can be utilized for the evaluation of contingencies depending on the defined system failure and the intent behind the studies. Adequacy indices were calculated using network flow, dc load flow and ac load flow methods and presented in Chapter 2. The computation time and the effect on the adequacy indices of the methods used are also discussed. It is shown in this chapter that the computation time depends on a number of factors of which the contingency level and the solution technique are the most important. It is not possible to obtain comprehensive system information using the network flow method or the dc load flow method. The results provided in Chapter 2 confirm the above statement and illustrate the importance of using the ac load flow solution technique in composite system reliability evaluation. 
The contribution from high level contingencies for a large system can be very significant and therefore can not be ignored in the calculation of representative indices for a composite power system. The number of possible contingencies and hence the computation time increases significantly as the level of contingency increases particularly when ac load flow is utilized as a solution technique. It is shown in Chapter 2 that not all possible contingencies create system problems. The CPU time and number of contingencies that create system problems for various contingency levels are illustrated in this chapter for the two test systems used throughout the thesis. It is found that only a small percentage of the total contingencies create system problems. It should, therefore, be emphasized that a significant amount of CPU time can be saved by preparing a contingency list using an appropriate method and running the contingencies from this list using the ac load flow method. This forms the basis of Chapter 3 .

The adequacy indices are calculated using ranking methods in Chapter 3. Three different performance indices are utilized and discussed in this chapter. The indices obtained using exhaustive contingency enumeration approach together with the decoupled load flow method, and the CPU time required to obtain these indices are considered as reference results. The efficiency and the accuracy of the ranking methods are then compared with these reference results. It is shown that for the RBTS, the ranking methods provide exactly the same indices as the reference values. The CPU time, however, is more than the reference CPU time. The CPU time for the IEEE-RTS is less with the ranking methods but the difference in the indices is significant. This is due to the masking effect explained in this chapter. In all the cases, it is found that the CPU time required for preparing the ranking list is minimum with $\mathrm{PI}_{\mathrm{V}}$, maximum with $\mathrm{PI}_{\mathrm{MWV}}$ and in between the two values with $\mathrm{PI}_{\mathrm{MW}}$. Two types of ranking lists and their merits and demerits are discussed in Chapter 3. The effects of consecutive success cutoff criterion on the CPU time are also presented in that chapter.

The inaccuracies in the results with all the PIs are due to masking error. This error can be reduced using a selection method instead of using ranking. The selection method for generator, line and combined generator and line outages is presented in Chapter 3. The results with the selection method are compared with those from ranking and with the 
reference results. It is shown that this method provides better results in terms of both computation time and accuracy. The selection method detects outages in terms of line overload and voltage violations. This selection method is further utilized for detecting all problem creating contingencies in subsequent chapters of the thesis.

The most significant indices in a composite system adequacy assessment are those related to the load curtailment. Concerns have been expresed in regard to the interpretation of these load curtailment indices. The composite generation and transmission system is classified in Chapter 4 into different system operating states, which provide a framework for incorporating security considerations in the reliability assessment. Quantitative indices for the system operating states are calculated considering line overloads as the basic system problem. Three constraint sets used in this thesis and an efficient method which avoids the recursive use of the Sherman-Morrison correction formula are also presented in this chapter.

The first objective, in the event of detecting a system problem, is to alleviate the problem by taking corrective action(s). The linear programming model developed for load curtailment and generation rescheduling and the solution techniques for the LP model are discussed in Chapter 4. In a practical system, the load does not stay at its peak value throughout the year. An evaluation of the system performance assuming a peak load model may therefore give highly pessimistic values for the reliability indices. These indices, referred to as annualized indices, are useful for comparing the performance of two or more systems but do not convey comprehensive information about the overall quantitative evaluation of the system. Modelling the system load as a multistep load provides more accurate results. A proper selection of the number of load states is primarily dictated by the shape of the load curve. In order to show the effect of a multistep load model and to calculate the annual indices, a 'seven-step' load model for the system load curve is considered. It is shown that the annual and the annualized indices are significantly different. The computation time for state evaluation can be reduced greatly by sorting the identical contingencies. The effect of only generator sorting, only line sorting and combined line and generator sorting on CPU times are illustrated. The saving in CPU time using sorting can be quite significant and, as illustrated, is more than $50 \%$ for the IEEE-RTS. 
The voltage magnitudes at some buses may deviate from acceptable limits due to the occurrence of certain contingencies. The voltage level at a bus must not deviate beyond the permissible limits to ensure a good quality of service. The acceptable voltage level at a bus, therefore, is an important factor in a reliability analysis. The indices for different system operating states were calculated in Chapter 5 by considering bus voltage magnitudes outside the acceptable limits to be a system problem. A LP model for voltage correction by changing the appropriate generation bus voltages and Q-load curtailment at different load buses is developed and presented using the MRBTS. Both annualized and annual indices considering the total constraint set are also presented in this chapter.

The two basic approaches to composite system reliability evaluation, namely enumeration and Monte Carlo simulation are combined to form a hybrid approach in Chapter 6. The indices for different system operating states are calculated using two hybrid techniques. The system situation is selected by random sampling and the sampled situation is assigned using the enumeration approach to an appropriate system operating state. It is shown that Method 1 does not provide satisfactory results. The results obtained using Method 2, however, converge to the corresponding analytical values when a suitable number of samples are used. The relative coefficient of variance $\alpha$ is used as a stopping criterion. There is no straight forward rule to select an $\alpha$ which will always provide satisfactory results within a reasonable number of samples and CPU time. A $\alpha$ equal to 0.05 gives a good compromise between acceptable indices and CPU time for the MRBTS. The required number of samples for $\alpha \leq 0.05$ is 45132 . Therefore, 50000 samples are considered to provide a reasonable estimate of the indices for the MRBTS. The CPU time increment for the IEEE-RTS increases quite significantly as the number of samples increases and 10000 samples for this system gives a $\alpha \leq 0.0326$. The required CPU time is also close to the analytical CPU time. This number of samples is therefore used for the IEEE-RTS to illustrate the effect of variance reduction techniques (VRT) on the indices of different operating states. The variance indicates the variability of the estimator around the expected value. Therefore, high variance essentially implies poor accuracy. The accuracy in estimating a reliability index can be improved by increasing the number of samples in the simulation or using an appropriate VRT. By applying an 
appropriate VRT, the precision of estimation for the same number of samples or, conversely the number of samples for the same precision can be reduced. Two VRTs, namely stratification after sampling and antithetic VRT are considered in Chapter 6. It is shown that the antithetic VRT gives more satisfactory results in terms of efficiency for the extreme emergency operating state. This conclusion, however, could be different for the other system states. Both annualized and annual indices are calculated and presented in this chapter.

A system operates without violating any constraints in both the normal and alert states. A system operating objective, therefore, is to operate with a high probability within these two system operating states. The complement of the sum of these two state probabilities is considered as a basic risk index in Chapter 7. This risk factor has been designated as the Composite System Operating State Risk (CSOSR). The utilization of this CSOSR as a criterion in simple system expansion planning to meet future load growth is illustrated in this chapter using the two test systems. It is shown that the location of additional generation and transmission facilities is very important in an HLII assessment. The addition of facilities at some location may make the system marginally more unreliable than the existing base system. In the case of the MRBTS, for example, the risk index with the addition of a line between buses 1 and 2 is greater than that of the base configuration up to a certain increase in peak load. It is shown that composite reinforcement with both generation and transmission gives better results than the simple addition of either generation or transmission facility for the MRBTS. The variation in the CSOSR index was less significant for the IEEE-RTS with additional generation compared to that for the MRBTS case. Reinforcement of transmission facility is found to have little effect on the risk index for the IEEE-RTS due to the fact that this system has an over sized transmission system. Composite reinforcement for the IEEE-RTS is therefore only slightly better than the generation addition case. The expansion plans illustrated in Chapter 7 are simple as the intention was to demonstrate the utilization of the new CSOSR. There are many other considerations which must be included in an actual expansion planning study. 
The system load does not stay at its peak load throughout a given period of time and therefore it is not economical to run all the generating units required to satisfy the peak load during the periods of low load. Generating units should be committed to service for a particular load level in such a way that the operating cost is minimized with a satisfactory level of reliability. A unit commitment method considering composite generation and transmission system is discussed in Chapter 8. The priority loading order for the generating units is used to commit units in service or to remove units from service depending on the load levels. It was found that the CSOSR is always higher than the unit commitment risk. The number of units committed for a particular load level from an HLI study, therefore, may or may not satisfy the CSOSR criterion. In a composite generation and transmission system, the units committed to service should satisfy both the unit commitment risk (HLI) and CSOSR (HLII). The number of units required for a given load level is a complex function of many factors, one of which is the lead time. The number of units committed for a specific load level can be minimized by reducing the lead time of additional generation provided other variables do not change. The effect of lead time and the number of generating units committed to the system on the probabilities of different system operating states are also presented in this chapter.

The utilization of the CSOSR index is illustrated using a relatively simple unit commitment framework in this thesis in which the lead times are considered to be the same for all generating units. Different lead times, derated states, etc. can be utilized in the calculation of system operating state indices. These state probabilities could be utilized as a criterion to extend the spinning reserve concepts established in HLI studies. It is shown that the CSOSR is always higher than the unit commitment risk and possibly two different acceptable risk values would provide a better risk interpretation. The determination of an acceptable value for CSOSR could include many other factors and could be the focus of further research activity. 


\section{REFERENCES}

1. Billinton, R. and Allan, R. N., "Power System Reliability in Perspective", IEE Electronics and Power, Mar 1984, pp. 231-236.

2. Billinton, R. and Allan, R. N., Reliability Evaluation of Power System, Plenum Press, New York, 1984.

3. Billinton R. and Tatla J. S., "Hierarchical Indices in System Adequacy Assessment", CEA Transactions, Engineering and Operating Division, Vol. 22, Part 3, Paper No. 83-SP-146, Mar 1983, pp. 1-14.

4. IEEE Task Force, "Reliability Indices for Use in Bulk Power System Adequacy Evaluation", IEEE Transactions on Power Apparatus and Systems, Vol. PAS-97, No. 4, Jul/Aug 1978, pp. 1097-1103.

5. Billinton, R., "Bibliography on the Application of Probability Methods in Power System Reliability Evaluation", IEEE Transactions on Power Apparatus and Systems, Vol. PAS-91, Mar/Apr 1972, pp. 649-660.

6. IEEE Committee Report, "Bibliography on the Application of Probability Methods in Power System Reliability Evaluation, 1971-1977", IEEE Transactions on Power Apparatus and Systems, Vol. PAS-97, Nov/Dec 1978, pp. 2235-2242.

7. Allan, R. N., Billinton, R. and Lee, S. H., "Bibliography on the Application of Probability Methods in Power System Reliability Evaluation, 1977-1982', IEEE Transactions on Power Apparatus and Systems, Vol. PAS-103, No. 2, Feb 1984, pp. 275-282.

8. Allan, R. N., Billinton, R., Shahidepour, S. M. and Singh, C., "Bibliography on the Application of Probability Methods in Power System Reliability Evaluation, 1982-1987', IEEE Transactions on Power Systems, Vol. PWRS-3, No. 4, Nov 1988, pp. 1555-1564.

9. IEEE Working Group on Distribution System Reliability of the Distribution Subcommittee of the Transmission And Distribution Committee, "Bibliography on Distribution System Reliability", IEEE Transactions on Power Apparatus and Systems, Vol. PAS-97, Mar/Apr 1978, pp. 545-548.

10. Mallard, S. A. and Thomas, V. C., "A Method for Calculating Transmission System Reliability", IEEE Transactions on Power Apparatus and Systems, Vol. PAS-87, Mar 1968, pp. 824-833.

11. Billinton, R., "Composite System Reliability Evaluation", IEEE Transactions on Power Apparatus and Systems, Vol. PAS-88, Apr 1969, pp. 276-280. 
12. Billinton, R. and Bhavaraju, M. P., "Transmission Planning Using a Reliability Criterion-Part I - A Reliability Criterion", IEEE Transactions on Power Apparatus and Systems, Vol. PAS-89, No. 1, Jan 1970, pp. 28-34.

13. Auge, J., Bergoungnoux, J., Dodu, J. C. and Pouget, J., "Probabilistic Study of a Transmission System Interconnection (Peru Model)", Proceedings of the 23rd Session, paper 32-16, Int. Conf. on Large Electric Systems, 1970.

14. Noferi, P. L. and Paris, L., "Quantitative Evaluation of Power System Reliability in Planning Studies", IEEE Transactions on Power Apparatus and Systems, Vol. PAS-91, No. 2, Mar/Apr 1972, pp. 611-618.

15. Paris, L., Reggiani, F. and Valtorta, M., "The Study of UHV System Reliability in Connection with its Structure and Component Characteristics", Proceedings of the 24th Session, paper 31-14, Int. Conf. on Large Electric Systems, 1972.

16. Bhavaraju, M. P. and Billinton, R., "Transmission System Reliability Methods", IEEE Transactions on Power Apparatus and Systems, Vol. PAS-91, No. 2, Mar/Apr 1972, pp. 628-637.

17. Noferi, P. L., Paris, L. and Salvaderi, L., "Monte Carlo Methods for Power System Reliability Evaluations in Transmission or Generation Planning", Proceedings, 1975 Reliability and Maintainability Symposium Washington, D.C., jan 1975, pp. 449-459.

18. Clancy, D. P., Gross, G. and Wu, F. F., "A Decomposition-Monte Carlo Approach for Multi-Area Reliability Calculations", Proc. 7th Power System Computation Conferance, Lausanne, Jul 1981, pp. 261-269.

19. Noferi, P. L. and Paris, L., "Effect of Voltage and Reactive Power Constraints on Power System Reliability", IEEE Transactions on Power Apparatus and Systems, Vol. PAS-94, Mar/Apr 1975, pp. 482-490.

20. Meliopoulos, A. P., Bakirtzis, A. and Webb R. P., "Bulk Power System Reliability Evaluation via Contingency Simulation", Proc. of the Southeastern Symp. on Systems Theory, Hunstville, AL, USA1983, pp. 210-214.

21. Meliopoulos, A. P., Bakirtzis, A. G. and Kovacs, R., "Power System Reliability Evaluation Using Stochastic Load Flows", IEEE Transactions on Power Apparatus and Systems, Vol. PAS-103, No. 5, May 1984, pp. 1084-1091.

22. El-Kady, M. A., El-Sobki, M. S. and Sinha, N. K., "Evaluation of Reliability of Large-Scale Power Systems Using Monte Carlo Method", Proc. IEEE ELECTRONICOM 85, Toronto, Canada1985, pp. 338-342.

23. Meliopoulos, A. P., Bakirtzis, A. G., Kovacs, R. R. and Beck, R. J., "'Bulk Power System Reliability Assessment Experience with the RECS Program", IEEE Transactions on Power Systems, Vol. PWRS-1, No. 3, Aug 1986, pp. 235-243.

24. Behnam-Guilani, K., "A Monte Carlo Method for Simultaneous Imort Capability Planning", Proc. of the 13th Inter-RAM Conference for the Electric Power Industry, Syracuse, Jun 1986, pp. 130-134.

25. Cress, S. L., "Statistical Analysis of Power Systems Using the Adjoint and Monte 
Carlo Methods", Proc. of the 1st Int. Symp. on Probabilistic Methods Applied to Electric Power Systems, Edited by S. Krishnasamy, Pergamon Press, Oxford, 1987, pp. 203-212.

26. Bertoldi, O., Salvaderi, L. and Scalcino, S., "Monte Carlo Approach in Planning Studies: An application to IEEE RTS", IEEE Transactions on Power Systems, Vol. PWRS-3, No. 3, Aug 1988, pp. 1146-1154.

27. Patton, A. D., "A Probability Method for Bulk Power System Security Assessment, I-Basic Concepts", IEEE Transactions on Power Apparatus and Systems, Vol. PAS-91, Jan/Feb 1972, pp. 54-61.

28. Billinton, R. and Singh, C., "Reliability Evaluation in Large Transmission Systems", IEEE Summer Meeting, Paper C72 475-2, San Francisco, Jul 1972.

29. Billinton, R., "Elements of Composite System Reliability Evaluation", CEA Transactions, Vol. 15, Part 2, Paper No. 76-SP-148, 1976.

30. Dandeno, P. L., Jorgensen, G. E., Puntel, W. R. and Ringlee, R. J., "A Program for Composite Bulk Power Electric System Adequacy Assessment", Transactions of the IEEE Conference on Reliability of Power Supply Systems, IEE Conference Publication No. 148, Feb 1977.

31. Marks, G. E., “A Method for Combining High Speed Contingency Load Flow Analysis with Stochastic Probability Methods to Calculate a Quantitative Measure of Overall Power System Reliability", IEEE Paper A78 053-1.

32. Billinton, R., Medicherla, T. K. P. and Sachdev, M. S., "Adequacy Indices for Composite Generation and Transmission System Reliability Evaluation", IEEE Trans. on Power Apparatus and System, Vol. PAS-98, No. 4, Jul/Aug 1979.

33. Billinton, R., Medicherla, T. K. P. and Sachdev, M. S., " Application of Common Cause Outage Models in Composite System Reliability Evaluation", IEEE Transactions on Power Apparatus and Systems, Vol. PAS-100, Jul 1981, pp. 3648-3657.

34. Billinton, R. and Medicherla, T. K. P., "Station Originated Multiple Outages in the Reliability Analysis of a Composite Generation and Transmission System", IEEE Transactions on Power Apparatus and Systems, Vol. PAS-100, No. 8, Aug 1981, pp. $3870-3878$.

35. Allan, R. N. and Adraktas, A. N., "Terminal Effects and Protection System Failures in Composite System Reliability Evaluation", IEEE Transactions on Power Apparatus and Systems, Vol. PAS-101, No. 12, Dec 1982, pp. 4557-4562.

36. Allan, R. N. and Ochoa, J. R., "'Modelling and Assessment of Station Originated Outages for Composite System Reliability Evaluation", IEEE Transactions on Power Systems, Vol. PWRS-3, No. 1, Feb 1988, pp. 158-165.

37. Mohamed, A. and Jasmon, G. B., "Realistic power System Security Algorithm", IEE Proceedings, Part C, Vol. 135, No. 2, Mar 1988, pp. 98-106.

38. IEEE Task Force, “IEEE Reliability Test System", IEEE Transactions on Power Apparatus and Systems, Vol. PAS-98, Nov/Dec 1979, pp. 2047-2054. 
39. Salvaderi, L. and Billinton, R., "A Comparison Between Two Fundamentally Different Approaches to Composite System Reliability Evaluation", IEEE Transactions on Power Apparatus and Systems, Vol. PAS-104, No. 12, Dec 1985, pp. 3486-3492.

40. Billinton, R., Allan, R. N. and Salvaderi, L., Applied Reliability Assessment in Electric Power Systems, IEEE Press, New York, 1990.

41. Billinton, R. and Khan, E., "Utilization of Contingency Selection Techniques in the Adequacy Evaluation of a Composite Generation and Transmission System", CEA Transsactions, Spring Meeting, 1988.

42. Billinton, R., Khan, E. and Agarwal, S.K., "Contingency Cutoff Criteria in Transmission System Adequacy Assessment", IEE Proceedings- $C$, Part $C$, Vol. 136, No. 4, Jul 1989, pp. 215-221.

43. Ejebe, G. C. and Wollenberg B. F., "Automatic Contingency Selection", IEEE Transactions on Power Apparatus and Systems, Vol. PAS-98, Jan/Feb 1979, pp. 97-109.

44. Mikolinnas, T. A. and Wollenberg, B. F., "An Advanced Contingency Selection Algorithm", IEEE Transactions on Power Apparatus and Systems, Vol. PAS-100, Feb 1981, pp. 608-617.

45. Irisarri, G. D., and Sasson, A. M., "An Automatic Contingency Selection Method for On-Line Security Analysis", IEEE Transactions on Power Apparatus and Systems, Vol. PAS-100, Apr 1981, pp. 1838-1844.

46. Halpin, T.F., Fischl, R. and Fink, R., "Analysis of Automatic Contingency Selection Algorithms", IEEE Transactions on Power Apparatus and Systems, Vol. PAS-103, May 1984, pp. 938-945.

47. EPRI Report, "Transmission System Reliability Methods - Mathematical Models, Computing Methods, and Results", Tech. report EPRI EL-2526, Power Technologies Inc., Schenectady, New York, Jul 1982.

48. Stott, B., Alsac, O. and Alvarado, F., "Analysis and Computational Improvements in Performance-Index Ranking Algorithms for Networks", International Journal of Electrical Power and Energy Systems, Vol. 7, No. 3, Jul 1985, pp. 154-160.

49. Dabbaghchi, I. and Irisarri, G., "AEP Automatic Contingency Selector", IEEE Transactions on Power Systems, Vol. PWRS-1, No. 2, May 1986, pp. 37-45.

50. Irisarri, G., Sasson, A. M. and Levner, D., "Automatic Contingency Selection for On-Line Security Analysis - Real-Time Tests", IEEE Transactions on Power Apparatus and Systems, Vol. PAS-98, No. 5, Sep/Oct 1979, pp. 1552-1559.

51. Puntel, W. R., Reppen, N. D., Ringlee, R. J., Platts, J. E., Ryan, W. A. and Sullivan, J., "Automated Method for Long-Range Planning of Transmission Networks", 1973 PICA Conference Proceedings, $\quad$ 73CHO 740-1 PWR, pp. 38-46.

52. Vemuri, S. and Usher, R. E., "On-Line Automatic Contingency Selection 
Algorithms", IEEE Transactions on Power Apparatus and Systems, Vol. PAS-102, Feb 1983, pp. 346-354.

53. Lauby, M. G., "Evaluation of a Local DC Load Flow Screening Method for Branch Contingency Selection of Overloads", IEEE Transactions on Power Systems, Vol. PWRS-3, Aug 1988, pp. 923-928.

54. Lauby, M. G., Mikolinnas, T. A. and Reppen, N. D., "Contingency Selection of Branch Outages Causing Voltage Problems", IEEE Transactions on Power Apparatus and Systems, Vol. PAS-102, Dec 1983, pp. 3899-3904.

55. Zaborsky, J., Whang, K. W. and Prasad, K., "Fast Contingency Evaluation Using Concentric Relaxation", IEEE Transactions on Power Apparatus and Systems, Vol. PAS-99, Jan/Feb 1980, pp. 28-36.

56. Albuyeh, F., Bose, A. and Heath, B., "Reactive Power Consideration in Automatic Contingency Selection", IEEE Transactions on Power Apparatus and Systems, Vol. PAS-101, Jan 1982, pp. 107-112.

57. Galiana, F. D., "Bound Estimates of the Severity of Line Outages in Power System Analysis and Ranking", IEEE Transactions on Power Apparatus and Systems, Vol. PAS-103, Sep 1984, pp. 2612-2622.

58. Nara, K. and et al, "On-Line Contingency Selection for Voltage Security Analysis", IEEE Transactions on Power Apparatus and Systems, Vol. PAS-104, Apr 1985, pp. 847-856.

59. Brandwajn, V., "Efficient Bounding Method for Linear Contingency Analysis", IEEE Transactions on Power Systems, Vol. PWRS-3, Feb 1988, pp. 38-43.

60. Stott, B. and Alsac, O., "Fast Decoupled Load Flow”, IEEE Transactions on Power Apparatus and Systems, Vol. PAS-93, May/Jun 1974, pp. 859-869.

61. Brandwajn, V. and Lauby, M. G., "Complete Bounding Method for AC Contingency Evaluation", presented at the IEEE/PES Meeting in Portland, Oregon, Jul 1988.

62. Ejebe, G., Van Meetern, H. and Wollenberg B. F., "Fast Contingency Screening and Evaluation for Voltage Security Analysis", IEEE Transactions on Power Systems, Vol. PWRS-3, No. 4, Nov 1988, pp. 1582-1590.

63. Endrenyi, J., Albrecht, P. F., Billinton, R., Marks, G. E., Reppen, N. D. and Salvaderi, L., "Bulk Power System Reliability Assessment-Why and How? Part I: Why?", IEEE Transactions on Power Apparatus and Systems, Vol. PAS-101, No. 9, Sep 1982, pp. 3439-3445.

64. Endrenyi, J., Albrecht, P. F., Billinton, R., Marks, G. E., Reppen, N. D. and Salvaderi, L., "Bulk Power System Reliability Assessment-Why and How? Part II: How?", IEEE Transactions on Power Apparatus and Systems, Vol. PAS-101, No. 9, Sep 1982, pp. 3446-3456.

65. Bhavaraju, M. P., Albrecht, P. F., Billinton, R., Reppen, N. D. and Ringlee, R. J., "Requirements for Composite System Reliability Evaluation Models", IEEE Transactions on Power Systems, Vol. PWRS-3, No. 2, Feb 1988, pp. 149-157. 
66. Kumar, S. and Billinton, R., "Adequacy Equivalents in Composite Power System Evaluation", IEEE Transactions on Power Systems, Vol. PWRS-3, No. 3, Aug 1988, pp. 1167-1173.

67. Billinton, R., Allan, R. N., Bhavaraju, M. P., Fong, C. C. Pereira, M. V. F., Ringlee, R. J., Salvaderi, L. and Sing, C., "Reliability Assessment of Composite Generation and Transmission Systems", IEEE Tutorial Book, IEEE Winter Power Meeting, Course Text 90EH0311-1-PWR, Jan 1990.

68. Billinton, R. and Khan, E., "A Comparison of Existing Computer Programs For Composite System Adequacy Evaluation', CEA Transactions, Engineering and Operating Division, Vol. 28, Part 3, Mar 1989.

69. Final Report, "Composite-System Reliability Evaluation: Phase 1 - Scoping Study", Tech. report EPRI EL-5290, Dec 1987.

70. Khan, E., "Fast Adequacy Evaluation of Composite Power Systems", Master's thesis, University Of Saskatchewan, Saskatoon, Canada, May 1988.

71. Dodu, J. C., "A Probabilistic Model for an Overall Study of Power Transmission Network Supply", Proceedings of the Power System Computation Conference, Gernoble, France, Sep 1972.

72. Fink, L. H. and Carlsen, K., “Operating Under Stress And Strain", IEEE Spectrum, Mar 1978.

73. Kumar, S., "Adequacy Evaluation of Composite Power Systems", Master's thesis, University Of Saskatchewan, Saskatoon, Canada, Jul 1984.

74. Kumar, S., Adequacy Evaluation in Composite Power Systems, $\mathrm{PhD}$ dissertation, University Of Saskatchewan, Saskatoon, Canada, Sep 1987.

75. Medicherla, T. K. P., Reliability Evaluation of Composite Generation and Transmission Systems, $\mathrm{PhD}$ dissertation, University Of Saskatchewan, Saskatoon, Canada, Dec 1978.

76. Billinton, R. and Kumar, S., "Pertinent Factors in the Adequacy Assessment of a Composite Generation and Transmission System", CEA Transactions, Engineering and Operating Division, Vol. 25, Part 3, Paper No. 86-SP-141,1986, pp. 1-32.

77. Ford, L. R., and Fulkerson, D. R., Flows in Networks, Princeton University Press, Princeton, N.J., 1962.

78. Stott, B., "Review of Load Flow Calculation Methods", Proc. IEEE, Vol. 62, Jul 1974, pp. 916-929.

79. Stagg, G. W. and El-Abiad, A. H., Computer Methods in Power System Analysis, Mcgraw-Hill Book Company, New York, 1968.

80. Sherman, J. and Morrison, W. J., "Adjustment of an Inverse Matrix corresponding To A Change In One Element Of A Given Matrix", An Math. Stat., Vol. 21, 1950, pp. 124. 
81. Billinton, R. and Khan, E., "Basic Elements in Reliability Evaluation of Composite Generation and Transmission Systems", Proceedings, II Symposium of Specialist in Electric Operational and Expansion Planning, Aug 21-25, Sao Paulo, Brazil, 1989.

82. Billinton, R. and Khan, E., "Contingency Selection in Composite System Adequacy Assessment", a paper presented in the IEEE/PES Summer Meeting, Minneapolis, Minnesota, Vol. Paper No. 90 SM 476-2-PWRS, July 15-19,1990.

83. Chowdhury, N. A., Spinning Reserve Assessment in Interconnected Generation Systems, PhD dissertation, University Of Saskatchewan, Saskatoon, Canada, Feb 1989.

84. Sullivan, R. L., Power System Planning, Mcgraw-Hill Book Company, New York, 1977.

85. Billinton, R., "Transmiossion System Reliability Models", EPRI Pub. \#EPRI WS-77-60, Mar 1978, pp. 2.10-2.17.

86. Patton, A. D., "Determination and Analysis of Data for Reliability Studies", IEEE Transactions on Power Apparatus and Systems, Vol. PAS-87, Jan 1968, pp. 84-100.

87. Serns, C., Duran, J. and Camargo, A., "A Model for Expansion Planning of Transmission Systems", IEEE Transactions on Power Apparatus and Systems, Vol. PAS-97, 1978, pp. 610-615.

88. Billinton, R. and Allan, R. N., Reliability Evaluation of Engineering Systems, Concepts and Techniques, Langmans London (England) / Plenum Press, New York, 1983.

89. Billinton, R. and Kumar, S., "Adequacy Evaluation of a Composite Power System - A Comparative Study of Existing Programs", CEA Transactions, Engineering and Operating Division, Vol. 24, Part 3, Paper No. 85-SP-141 , Mar 1985, pp. 1-14.

90. Medicherla, T. K. P. Billinton, R. and Sachdev, M. S., "Generation Rescheduling and Load Shedding to Alleviate Line Overloads - Analysis", IEEE Transactions on Power Apparatus and Systems, Vol. PAS-98, Nov/Dec 1979, pp. 1876-1884.

91. Billinton, R., Khan, E. and et al, "A Reliability Test System for Educational Purposes- Basic Data", IEEE Transactions on Power Systems, Vol. PWRS-3, No. 4, Aug 1989, pp. 1238-1244.

92. Billinton, R., Khan, E., "Quantitative Assessment of Composite Generation and Transmission Systems", A Paper Presented at the 10th Power Systems Computation Conference, PSCC, Aug 19-24, Graz, Austria 1990.

93. Billinton, R. and Allan, R. N., Reliability Assessment of Large Electric Power Systems, Kluwer Academic Publishers, Boston, 1984.

94. Wasley, R. G. and Daneshdoost, M., "Identification and Ranking of Critical Contingencies in Dependent Variable Space", IEEE Transactions on Power Apparatus and Systems, Vol. PAS-102, No. 4, Apr 1983, pp. 881-892. 
95. Tinney, W. F. and Powell, W. L., "Comparison of Matrix Inversion and Sparse Triangular Factorization for Solution of Power Network Problems", U.S.A/Romania Joint Seminar on Electric Power, Bucharest, June 1974.

96. Dandeno, P. L., Jorgensen, P. L., Puntel, W. R. and Ringlee, R. J., “A Program for Composite Bulk Power Electric System Adequacy Assessment", IEEE International Conference on Reliability of Power Supply Systems, London, Feb 1977.

97. Billinton, R. and Oteng-Adjei, J., "Utilization Of Interupted Energy Assessment Rates In Generation And Transmission System Planning", a paper presented in the IEEE/PES 1990 Summer Meeting, Minneapolis, Minnesota, Jul 15-19, 1990.

98. Sposito, V. A., Linear and Nonlinear Programming, The IOWA State University Press, Ames, Iowa, 1975.

99. William,H. P., Brian, P. F., Saul, A. T. and William, T. V., Numerical Recipes in C The Art of Scientific Computing, Cambridge University Press, New York, 1988.

100. Felix, F. W. and Sadatoshi, K., "Steady-State Security Regions of Power Systems", IEEE Transactions on Circuits and Systems, Vol. CAS-29, Nov 1982, pp. 703-711.

101. Arrillaga, J., Arnold, C. P. and Harker, B.J., Computer Modelling of Electrical Power Systems, John Wiely and Sons, New York, 1983.

102. Endrenyi, J., Reliability Modelling in Electric Power Systems, John Wiley and Sons, New York, 1978.

103. Byron, J. T. Morgan, Elements of Simulation, Chapman and Hall, London, New York, 1984.

104. Jerry Banks, John S. Carson, II., Discrete-Event System Simulation, Prentice-Hall Inc., Englewood Cliffs, New Jersy, 1984.

105. Cunha, S. H. F., et al, "Composite Generation and Transmission Reliability Evaluation in Large Hydroelectric Systems", IEEE Transactions on Power Apparatus and Systems, Vol. PAS-104, No. 10, Oct 1985, pp. 2657-2663.

106. Cochran, W. G., Sampling Techniques, Wiely, Second Edition, New York, 1963.

107. Billinton, R., Allan, R. N. and Abdel-Gaward, N. M. K., "The IEEE Reliability Test System: Extensions to and Evaluation of the Generation System", IEEE Transactions on Power Systems, Vol. PWRS-1, No. 4, Nov 1986, pp. 1-7.

108. Anstine, L. T., Burke, R. E., Casey, J. E., Holgate, R., John, R. S. and Stewart, H. G., "Application of Probability Methods to the Determination of Spinning Reserve Requirements for the Pennsylvania-NewJersey-Maryland Interconnection", IEEE Transactions on Power Apparatus and Systems, Vol. PAS-82, 1963, pp. 726-735. 


\title{
A. Data of the 6 bus RBTS
}

\author{
Base MVA $=100$
}

Table A.1: Bus data

\begin{tabular}{|c|c|c|c|c|c|c|c|c|}
\hline Bus & $\begin{array}{l}\text { Lo } \\
\text { Active }\end{array}$ & $\begin{array}{l}\text { (p.u.) } \\
\text { Reactive }\end{array}$ & $P_{G}$ & $\mathrm{Q}_{\text {Max }}$ & $\mathrm{Q}_{\mathrm{Min}}$ & $\mathrm{V}_{0}$ & $\mathrm{~V}_{\text {Max }}$ & $\mathrm{V}_{\text {Min }}$ \\
\hline 1 & 0.000 & 0.000 & 1.000 & 0.50 & -0.40 & 1.05 & 1.05 & 0.97 \\
\hline 2 & 0.200 & 0.000 & 1.200 & 0.75 & -0.40 & 1.05 & 1.05 & 0.97 \\
\hline 3 & 0.850 & 0.000 & 0.000 & 0.00 & 0.00 & 1.00 & 1.05 & 0.97 \\
\hline 4 & 0.400 & 0.000 & 0.000 & 0.00 & 0.00 & 1.00 & 1.05 & 0.97 \\
\hline 5 & 0.200 & 0.000 & 0.000 & 0.00 & 0.00 & 1.00 & 1.05 & 0.97 \\
\hline 6 & 0.200 & 0.000 & 0.000 & 0.00 & 0.00 & 1.00 & 1.05 & 0.97 \\
\hline
\end{tabular}

Table A.2: Line data

\begin{tabular}{llllllllll}
\hline $\begin{array}{c}\text { Line } \\
\text { No. }\end{array}$ & $\begin{array}{c}\text { Buses } \\
\text { I }\end{array}$ & $\mathrm{J}$ & $\mathrm{R}$ & $\mathrm{X}$ & $\mathrm{B} / 2$ & Tap & $\begin{array}{c}\text { Current } \\
\text { Rating } \\
\text { (p.u.) }\end{array}$ & $\begin{array}{c}\text { Failures } \\
\text { Per Year }\end{array}$ & $\begin{array}{c}\text { Repair } \\
\text { Time } \\
\text { (hours) }\end{array}$ \\
\hline 1 & 1 & 3 & 0.0342 & 0.1800 & 0.0106 & 1.00 & 0.85 & 1.500 & 10.00 \\
2 & 2 & 4 & 0.1140 & 0.6000 & 0.0352 & 1.00 & 0.71 & 5.000 & 10.00 \\
3 & 1 & 2 & 0.0912 & 0.4800 & 0.0282 & 1.00 & 0.71 & 4.000 & 10.00 \\
4 & 3 & 4 & 0.0228 & 0.1200 & 0.0071 & 1.00 & 0.71 & 1.000 & 10.00 \\
5 & 3 & 5 & 0.0228 & 0.1200 & 0.0071 & 1.00 & 0.71 & 1.000 & 10.00 \\
6 & 1 & 3 & 0.0342 & 0.1800 & 0.0106 & 1.00 & 0.85 & 1.500 & 10.00 \\
7 & 2 & 4 & 0.1140 & 0.6000 & 0.0352 & 1.00 & 0.71 & 5.000 & 10.00 \\
8 & 4 & 5 & 0.0228 & 0.1200 & 0.0071 & 1.00 & 0.71 & 1.000 & 10.00 \\
9 & 5 & 6 & 0.0228 & 0.1200 & 0.0071 & 1.00 & 0.71 & 1.000 & 10.00 \\
\hline
\end{tabular}


Table A.3: Generator data

\begin{tabular}{ccccc}
\hline $\begin{array}{c}\text { Unit } \\
\text { No. }\end{array}$ & $\begin{array}{l}\text { Bus } \\
\text { No. }\end{array}$ & $\begin{array}{c}\text { Rating } \\
\text { (MW) }\end{array}$ & $\begin{array}{c}\text { Failures } \\
\text { per Year }\end{array}$ & $\begin{array}{c}\text { Repair } \\
\text { Time (hours) }\end{array}$ \\
\hline & 1 & 40.00 & 6.0000 & 45.00 \\
1 & 1 & 40.00 & 6.0000 & 45.00 \\
3 & 1 & 10.00 & 4.0000 & 45.00 \\
4 & 1 & 20.00 & 5.0000 & 45.00 \\
5 & 2 & 5.00 & 2.0000 & 45.00 \\
6 & 2 & 5.00 & 2.0000 & 45.00 \\
7 & 2 & 40.00 & 3.0000 & 60.00 \\
8 & 2 & 20.00 & 2.4000 & 55.00 \\
9 & 2 & 20.00 & 2.4000 & 55.00 \\
10 & 2 & 20.00 & 2.4000 & 55.00 \\
11 & 2 & 20.00 & 2.4000 & 55.00 \\
\hline
\end{tabular}




\section{B. Data of the IEEE-RTS}

Base MVA $=100$

Table B.1: Bus data

\begin{tabular}{|c|c|c|c|c|c|c|c|c|}
\hline \multirow[t]{2}{*}{ Bus } & \multicolumn{2}{|c|}{ Load (p.u.) } & \multirow[t]{2}{*}{$P_{G}$} & \multirow[t]{2}{*}{$\mathrm{Q}_{\operatorname{Max}}$} & \multirow[t]{2}{*}{$\mathrm{Q}_{\mathrm{Min}}$} & \multirow[t]{2}{*}{$\mathrm{V}_{0}$} & \multirow[t]{2}{*}{$\mathrm{V}_{\mathrm{Max}}$} & \multirow[t]{2}{*}{$\mathrm{V}_{\text {Min }}$} \\
\hline & Active & Reactive & & & & & & \\
\hline 1 & 1.080 & 0.220 & 1.720 & 1.20 & -0.75 & 1.02 & 1.05 & 0.95 \\
\hline 2 & 0.970 & 0.200 & 1.720 & 1.20 & -0.75 & 1.02 & 1.05 & 0.95 \\
\hline 3 & 1.800 & 0.370 & 0.000 & 0.00 & 0.00 & 1.00 & 1.05 & 0.95 \\
\hline 4 & 0.740 & 0.150 & 0.000 & 0.00 & 0.00 & 1.00 & 1.05 & 0.95 \\
\hline 5 & 0.710 & 0.140 & 0.000 & 0.00 & 0.00 & 1.00 & 1.05 & 0.95 \\
\hline 6 & 1.360 & 0.280 & 0.000 & 0.00 & 0.00 & 1.00 & 1.05 & 0.95 \\
\hline 7 & 1.250 & 0.250 & 3.000 & 2.70 & 0.00 & 1.02 & 1.05 & 0.95 \\
\hline 8 & 1.710 & 0.350 & 0.000 & 0.00 & 0.00 & 1.00 & 1.05 & 0.95 \\
\hline 9 & 1.750 & 0.360 & 0.000 & 0.00 & 0.00 & 1.00 & 1.05 & 0.95 \\
\hline 10 & 1.950 & 0.400 & 0.000 & 0.00 & 0.00 & 1.00 & 1.05 & 0.95 \\
\hline 11 & 0.000 & 0.000 & 0.000 & 0.00 & 0.00 & 1.00 & 1.05 & 0.95 \\
\hline 12 & 0.000 & 0.000 & 0.000 & 0.00 & 0.00 & 1.00 & 1.05 & 0.95 \\
\hline 13 & 2.650 & 0.540 & 5.500 & 3.60 & 0.00 & 1.02 & 1.05 & 0.95 \\
\hline 14 & 1.940 & 0.390 & 0.000 & 3.00 & -0.75 & 1.02 & 1.05 & 0.95 \\
\hline 15 & 3.170 & 0.640 & 2.100 & 1.65 & -0.75 & 1.02 & 1.05 & 0.95 \\
\hline 16 & 1.000 & 0.200 & 1.450 & 1.20 & -0.75 & 1.02 & 1.05 & 0.95 \\
\hline 17 & 0.000 & 0.000 & 0.000 & 0.00 & 0.00 & 1.00 & 1.05 & 0.95 \\
\hline 18 & 3.330 & 0.680 & 4.000 & 3.00 & -0.75 & 1.02 & 1.05 & 0.95 \\
\hline 19 & 1.810 & 0.370 & 0.000 & 0.00 & 0.00 & 1.00 & 1.05 & 0.95 \\
\hline 20 & 1.280 & 0.260 & 0.000 & 0.00 & 0.00 & 1.00 & 1.05 & 0.95 \\
\hline 21 & 0.000 & 0.000 & 3.500 & 3.00 & -0.75 & 1.02 & 1.05 & 0.95 \\
\hline 22 & 0.000 & 0.000 & 2.500 & 1.45 & -0.90 & 1.02 & 1.05 & 0.95 \\
\hline 23 & 0.000 & 0.000 & 6.600 & 4.50 & -1.75 & 1.02 & 1.05 & 0.95 \\
\hline 24 & 0.000 & 0.000 & 0.000 & 0.00 & 0.00 & 1.00 & 1.05 & 0.95 \\
\hline
\end{tabular}


Table B.2: Line data

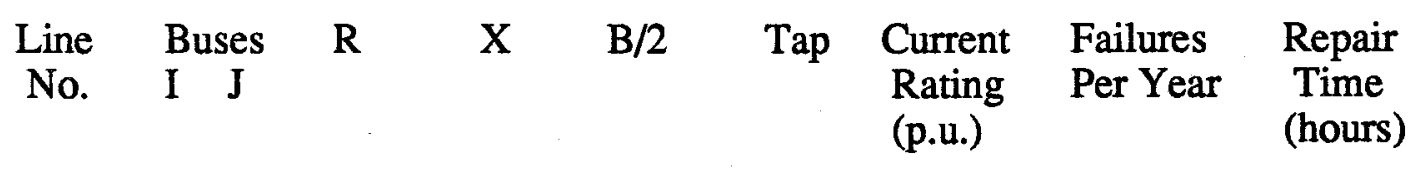

\begin{tabular}{|c|c|c|c|c|c|c|c|c|c|}
\hline 1 & 1 & 2 & 0.0026 & 0.0139 & 0.2306 & 1.00 & 1.93 & 0.240 & 16.00 \\
\hline 2 & 1 & 3 & 0.0546 & 0.2112 & 0.0286 & 1.00 & 2.08 & 0.510 & 10.00 \\
\hline 3 & 1 & 5 & 0.0218 & 0.0845 & 0.0115 & 1.00 & 2.08 & 0.330 & 10.00 \\
\hline 4 & 2 & 4 & 0.0328 & 0.1267 & 0.0172 & 1.00 & 2.08 & 0.390 & 10.00 \\
\hline 5 & 2 & 6 & 0.0497 & 0.1920 & 0.0260 & 1.00 & 2.08 & 0.480 & 10.00 \\
\hline 6 & 3 & 9 & 0.0308 & 0.1190 & 0.0161 & 1.00 & 2.08 & 0.380 & 10.00 \\
\hline 7 & 3 & 24 & 0.0023 & 0.0839 & 0.0000 & 1.00 & 5.10 & 0.020 & 768.00 \\
\hline 8 & 4 & 9 & 0.0268 & 0.1037 & 0.0141 & 1.00 & 2.08 & 0.360 & 10.00 \\
\hline 9 & 5 & 10 & 0.0228 & 0.0883 & 0.0120 & 1.00 & 2.08 & 0.340 & 10.00 \\
\hline 10 & 6 & 10 & 0.0139 & 0.0605 & 1.2295 & 1.00 & 1.93 & 0.330 & 35.00 \\
\hline 11 & 7 & 8 & 0.0159 & 0.0614 & 0.0166 & 1.00 & 2.08 & 0.300 & 10.00 \\
\hline 12 & 8 & 9 & 0.0427 & 0.1651 & 0.0224 & 1.00 & 2.08 & 0.440 & 10.00 \\
\hline 13 & 8 & 10 & 0.0427 & 0.1651 & 0.0224 & 1.00 & 2.08 & 0.440 & 10.00 \\
\hline 14 & 9 & 11 & 0.0023 & 0.0839 & 0.0000 & 1.00 & 6.00 & 0.020 & 768.00 \\
\hline 15 & 9 & 12 & 0.0023 & 0.0839 & 0.0000 & 1.00 & 6.00 & 0.020 & 768.00 \\
\hline 16 & 10 & 11 & 0.0023 & 0.0839 & 0.0000 & 1.00 & 6.00 & 0.020 & 768.00 \\
\hline 17 & 10 & 12 & 0.0023 & 0.0839 & 0.0000 & 1.00 & 6.00 & 0.020 & 768.00 \\
\hline 18 & 11 & 13 & 0.0061 & 0.0476 & 0.0500 & 1.00 & 6.00 & 0.400 & 11.00 \\
\hline 19 & 11 & 14 & 0.0054 & 0.0418 & 0.0440 & 1.00 & 6.00 & 0.390 & 11.00 \\
\hline 20 & 12 & 13 & 0.0061 & 0.0476 & 0.0500 & 1.00 & 6.00 & 0.400 & 11.00 \\
\hline 21 & 12 & 23 & 0.0124 & 0.0966 & 0.1015 & 1.00 & 6.00 & 0.520 & 11.00 \\
\hline 22 & 13 & 23 & 0.0111 & 0.0865 & 0.0909 & 1.00 & 6.00 & 0.490 & 11.00 \\
\hline 23 & 14 & 16 & 0.0050 & 0.0389 & 0.0409 & 1.00 & 6.00 & 0.380 & 11.00 \\
\hline 24 & 15 & 16 & 0.0022 & 0.0173 & 0.0364 & 1.00 & 6.00 & 0.330 & 11.00 \\
\hline 25 & 15 & 21 & 0.0063 & 0.0490 & 0.0515 & 1.00 & 6.00 & 0.410 & 11.00 \\
\hline 26 & 15 & 21 & 0.0063 & 0.0490 & 0.0515 & 1.00 & 6.00 & 0.410 & 11.00 \\
\hline 27 & 15 & 24 & 0.0067 & 0.0519 & 0.0546 & 1.00 & 6.00 & 0.410 & 11.00 \\
\hline 28 & 16 & 17 & 0.0033 & 0.0259 & 0.0273 & 1.00 & 6.00 & 0.350 & 11.00 \\
\hline 29 & 16 & 19 & 0.0030 & 0.0231 & 0.0243 & 1.00 & 6.00 & 0.340 & 11.00 \\
\hline 30 & 17 & 18 & 0.0018 & 0.0144 & 0.0152 & 1.00 & 6.00 & 0.320 & 11.00 \\
\hline 31 & 17 & 22 & 0.0135 & 0.1053 & 0.1106 & 1.00 & 6.00 & 0.540 & 11.00 \\
\hline 32 & 18 & 21 & 0.0033 & 0.0259 & 0.0273 & 1.00 & 6.00 & 0.350 & 11.00 \\
\hline 33 & 18 & 21 & 0.0033 & 0.0259 & 0.0273 & 1.00 & 6.00 & 0.350 & 11.00 \\
\hline 34 & 19 & 20 & 0.0051 & 0.0396 & 0.0417 & 1.00 & 6.00 & 0.380 & 11.00 \\
\hline 35 & 19 & 20 & 0.0051 & 0.0396 & 0.0417 & 1.00 & 6.00 & 0.380 & 11.00 \\
\hline 36 & 20 & 23 & 0.0028 & 0.0216 & 0.0228 & 1.00 & 6.00 & 0.340 & 11.00 \\
\hline 37 & 20 & 23 & 0.0028 & 0.0216 & 0.0228 & 1.00 & 6.00 & 0.340 & 11.00 \\
\hline 38 & 21 & 22 & 0.0087 & 0.0678 & 0.0712 & 1.00 & 6.00 & 0.450 & 11.00 \\
\hline
\end{tabular}


Table B.3: Generator data

\begin{tabular}{|c|c|c|c|c|}
\hline $\begin{array}{l}\text { Unit } \\
\text { No. }\end{array}$ & $\begin{array}{l}\text { Bus } \\
\text { No. }\end{array}$ & $\begin{array}{l}\text { Rating } \\
\text { (MW) }\end{array}$ & $\begin{array}{l}\text { Failures } \\
\text { per Year }\end{array}$ & $\begin{array}{c}\text { Repair } \\
\text { Time (hours) }\end{array}$ \\
\hline 1 & 22 & 50.00 & 4.42000 & 20.00 \\
\hline 2 & 22 & 50.00 & 4.42000 & 20.00 \\
\hline 3 & 22 & 50.00 & 4.42000 & 20.00 \\
\hline 4 & 22 & 50.00 & 4.42000 & 20.00 \\
\hline 5 & 22 & 50.00 & 4.42000 & 20.00 \\
\hline 6 & 22 & 50.00 & 4.42000 & 20.00 \\
\hline 7 & 15 & 12.00 & 2.98000 & 60.00 \\
\hline 8 & 15 & 12.00 & 2.98000 & 60.00 \\
\hline 9 & 15 & 12.00 & 2.98000 & 60.00 \\
\hline 10 & 15 & 12.00 & 2.98000 & 60.00 \\
\hline 11 & 15 & 12.00 & 2.98000 & 60.00 \\
\hline 12 & 15 & 155.00 & 9.13000 & 40.00 \\
\hline 13 & 7 & 100.00 & 7.30000 & 50.00 \\
\hline 14 & 7 & 100.00 & 7.30000 & 50.00 \\
\hline 15 & 7 & 100.00 & 7.30000 & 50.00 \\
\hline 16 & 13 & 197.00 & 9.22000 & 50.00 \\
\hline 17 & 13 & 197.00 & 9.22000 & 50.00 \\
\hline 18 & 13 & 197.00 & 9.22000 & 50.00 \\
\hline 19 & 1 & 20.00 & 19.47000 & 50.00 \\
\hline 20 & 1 & 20.00 & 19.47000 & 50.00 \\
\hline 21 & 1 & 76.00 & 4.47000 & 40.00 \\
\hline 22 & 1 & 76.00 & 4.47000 & 40.00 \\
\hline 23 & 2 & 20.00 & 19.47000 & 50.00 \\
\hline 24 & 2 & 20.00 & 19.47000 & 50.00 \\
\hline 25 & 2 & 76.00 & 4.47000 & 40.00 \\
\hline 26 & 2 & 76.00 & 4.47000 & 40.00 \\
\hline 27 & 23 & 155.00 & 9.13000 & 40.00 \\
\hline 28 & 23 & 155.00 & 9.13000 & 40.00 \\
\hline 29 & 23 & 350.00 & 7.62000 & 100.00 \\
\hline 30 & 18 & 400.00 & 7.96000 & 150.00 \\
\hline 31 & 21 & 400.00 & 7.96000 & 150.00 \\
\hline 32 & 16 & 155.00 & 9.13000 & 40.00 \\
\hline
\end{tabular}




\section{RANDOM NUMBER GENERATORS}

\section{C.1. RANDOM NUMBER GENERATION}

Any method based on randomness to solve a problem is called Monte Carlo simulation method. Therefore, random numbers are a necessary basic ingredient in the simulation study. There are many methods available for generating random numbers such as using tables, physical devices and pseudo-random numbers. A sequence of random numbers must have two important properties known as

1. uniformity and

2. independence.

The pseudo-random numbers are usually used in the simulation studies. This means that the random numbers are produced by a known method. The objective of any random number generation scheme is to produce a sequence of numbers between zero and one which simulate the ideal properties of uniform distribution and independence as closely as possible. There are numerous methods that can be used to generate the random numbers. The random number generator algorithm should have the following important characteristics

1. the routine should be fast

2. the routine should not require a lot of core storage

3. the routine should have sufficiently long cycle

4. the random number should be replicable

5. the generated random number should closely approximate the ideal properties of uniformity and independence

The most common methods for generating random numbers are

1. linear congruential method 
2. constant multiple technique

3. additive congruential method

4. midsquared method

5. mid product technique

The linear congruential method is the most widely used technique today. This method is, therefore, discussed briefly in the next subsection.

\section{C.1.1. Linear Congruential Method}

The linear congruential method produces a sequence of integers $X_{1}, X_{2}, \ldots$. between zero and $\mathrm{m}-1$ according to the following recursive relationship

$$
\mathrm{X}_{\mathrm{i}+1}=(\mathrm{bX}+\mathrm{i}) \bmod \mathrm{m} \mathrm{i}=0,1,2, \ldots
$$

The initial value of $\mathrm{X}_{0}$ is called the seed, $\mathrm{b}$ is called the constant multiplier, $\mathrm{c}$ is the increment and $\mathrm{m}$ is the modulus. The selection of the values for $\mathrm{b}, \mathrm{c}, \mathrm{m}$ and $\mathrm{X}_{0}$ drastically effects the statistical properties and the cycle length. If $c \neq 0$ in Equation C. 1 , the form is called mixed congruential method and when $c=0$, the form is known as the multiplicative congruential method. In the mixed congruential method the maximum period can be achieved by the following proper choice of $b, c, m$ and $X_{0}$

1. $\mathrm{c}$ is relatively prime to $\mathrm{m}$

2. (b-1) is a multiple of every prime number that divides $m$

3. (b-1) is a multiple of 4 if $m$ is a multiple of 4 .

If $m=2^{a}$, relation 3 will imply that $b=4 k+1$ for positive integral $k$. Such a value of $b$ also satisfy relation 2 . When $m=2^{a}$, relation 1 is easily obtained by setting $c$ to be any odd positive constant. The commonly used case is $m=2^{\mathrm{a}}, \mathrm{b}=4 \mathrm{k}+1$ and $\mathrm{c}$ odd where $\mathrm{k}, \mathrm{c}$, and a are positive integers.

As noted earlier, the multiplicative congruential generator can be written from Equation C.1 as

$$
\mathrm{X}_{\mathrm{i}+1}=\mathrm{bX} \mathrm{X}_{\mathrm{i}} \bmod \mathrm{m} \mathrm{i}=0,1,2, \ldots
$$

A full period can not be achieved with this method. For $m$ a power of 2 , say $m=2^{a}$, the 
largest possible period is $\mathrm{P}=\mathrm{m} / 4=2^{\mathrm{a}-2}$. This maximum period can be obtained if the following conditions are satisfied

1. the seed is an odd number

2. the multiplier $b$ is given by $b=8 k \pm 3$ where $k$ is any integer.

The computational efficiency of multiplicative congruential method is better than that of the mixed congruential method, however, it is not possible to obtain the full cycle in the multiplicative case. In order to obtain a full cycle length, the following random number generator has been used for the simulation studies in this research.

\section{C.1.2. Prime Number Multiplicative Congruential Generator}

When the modulus $\mathrm{m}$ is a prime number, the maximum possible period length is $\mathrm{m}-1$. If the integer word-length of a computer is $2^{31}$, the largest prime number is $2^{31}-1$. The multiplicative generator given above can be modified as follows: Two integers $\mathrm{N}$ and $M$ are chosen such that:

$1.0<\mathrm{N}<\mathrm{m}$

$2.0 \leq \mathrm{M}<\mathrm{b}$

3. $\mathrm{bN}+\mathrm{M}=\mathrm{m}$

With the above selected constants, the prime-number multiplicative congruential generator can be written as

$$
\mathrm{X}_{\mathrm{i}+1}^{\prime}=\mathrm{b}\left[\mathrm{X}_{\mathrm{i}} \bmod \mathrm{N}\right]-\mathrm{k}_{1} \mathrm{M}
$$

where $k_{1}=\left[X_{i} / N\right]$ and the square bracket denotes the largest positive integer in $\left[X_{i} / N\right]$.

$$
X_{i+1}=\left\{\begin{array}{l}
X_{i+1}^{\prime} \text { if } X_{i+1}^{\prime} \geq 0 \\
X_{i+1}^{\prime}+m \text { if } X_{i+1}^{\prime}<0
\end{array}\right.
$$

The following values of the constants are used in this random number generator

1. $b=16807$,

2. $\mathrm{N}=127773$,

3. $\mathrm{M}=2836$ and

4. $m=2^{31}-1$.

The statistical tests for uniformity and independence are satisfactory with the above choice of the constants. 